\title{
STUDY OF DIVERSITY TECHNIQUES FOR ULTRA-WIDEBAND GROUND PENETRATING RADAR
}

\author{
DELPHINE HELENA NATASHA MARPAUNG
}

School of Electrical and Electronic Engineering

A thesis submitted to the Nanyang Technological University in partial fulfillment of the requirement for the degree of

Doctor of Philosophy 


\section{STATEMENT OF ORIGINALITY}

I hereby certify that the work embodied in this thesis is the result of original research and has not been submitted for a higher degree to any other University or Institution.

Date

Delphine Helena Natasha Marpaung 
DEDICATION

To my family, 


\section{Acknowledgements}

First, I would like to express my sincere appreciation to the Nanyang Technological University (NTU), Singapore, for providing the full Research Scholarship to support my PhD study.

I would like to express my sincere gratitude to Professor LU Yilong who has been instrumental in guiding my research, providing insightful advice and regular supervision of my work. His encouraging words and invaluable support through the years have kept me motivated.

In addition, throughout this journey I am eternally grateful for the constant love and support of my family and friends who bring great joy to my life. 


\begin{abstract}
Ground penetrating radar (GPR) is a subsurface imaging method that utilizes an electromagnetic wave in microwave frequency domain. GPR has very wide applications in many different fields, such as civil engineering, archaeology, military, geophysics, and etc. This thesis presents a study of emerging GPR techniques that incorporate spatial diversity waveform diversity, and polarization diversity techniques on GPR systems in addition to ultra-wideband (UWB) technologies. The main contributions of this study are from the following three aspects.
\end{abstract}

Firstly, MIMO configuration has been evaluated for UWB GPR system. The performance of MIMO array is compared with single-input single-output (SISO) and single-input multi-output (SIMO) arrays over various applications and migration methods of GPR. It is shown that MIMO configuration improves the resolution and signal to noise ratio (SNR), reduces the side-lobe level and image artefacts, and has better capability in imaging various target shapes. In addition, an efficient autofocusing method has been proposed for MIMO configuration. This method utilizes migration to zero-offset operator that transform a MIMO data into its equivalent SISO data. The proposed method successfully reduces the computational complexity in focusing MIMO data while preserving the performance improvement provided by MIMO configuration.

Secondly, a study of waveform diversity on UWB GPR has been performed. The waveform diversity is implemented by collecting the data with 4 different impulse waveforms. A processing method that combines these datasets has been proposed to distinguish several target materials on some target shapes.

Thirdly, the implementation of polarimetric decomposition methods on UWB GPR has been investigated. Several incoherent and coherent polarimetry 
decompositions from synthetic aperture radar are applied to UWB GPR data. The performance of these methods is evaluated on several target models based on utilities mapping application. The results show that most of the polarimetry decomposition methods provide colour information that can be used to distinguish the shape of the target. The incoherent decompositions outperform the coherent decompositions in providing cleaner images, but at the expense of higher computational complexity. Finally, the polarimetry characteristic of MIMO and SISO configuration are compared. The simulation results show that both configurations provide almost similar polarimetry characteristic.

The performance of MIMO configuration and waveform diversity method are investigated based on simulation data. Meanwhile, the polarimetry techniques are verified experimentally in addition to the analysis on simulation data. 


\section{TABLE OF CONTENTS}

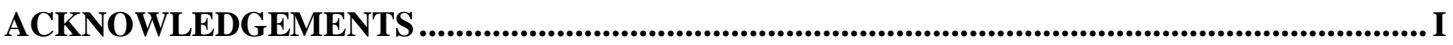

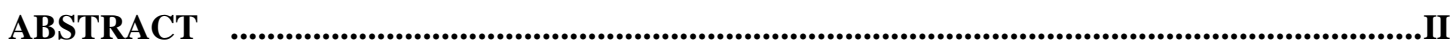

TABLE OF CONTENTS................................................................................................................. IV

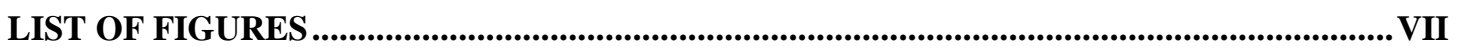

LIST OF TABLES .................................................................................................................................

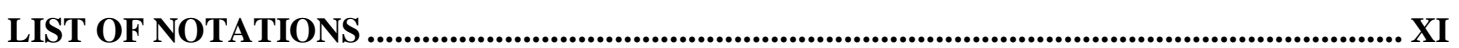

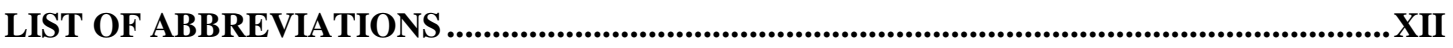

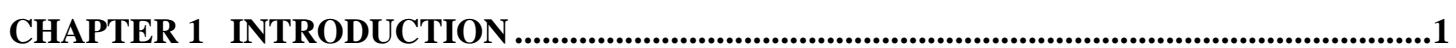

1.1 Overview of Ultra-Wideband Ground Penetrating Radar System ......................... 1

1.2 Diversities In Ultra-Wideband Ground Penetrating RADAR ...................................... 4

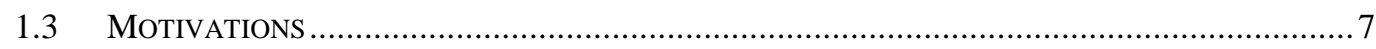

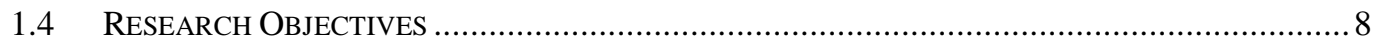

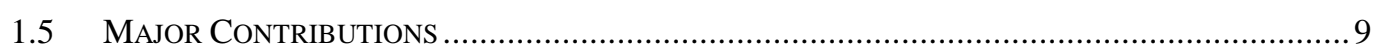

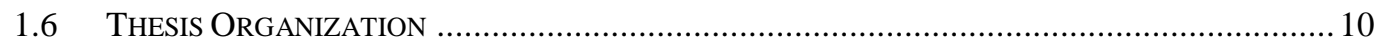

\section{CHAPTER 2 FUNDAMENTALS OF ULTRA-WIDEBAND GROUND PENETRATING}

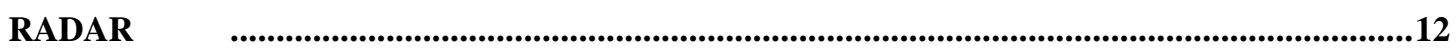

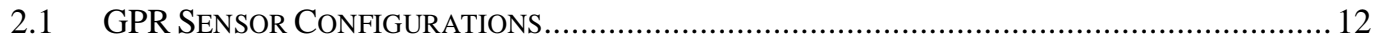

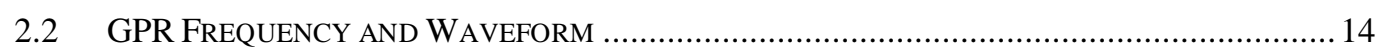

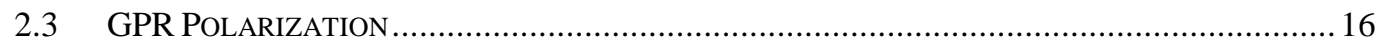

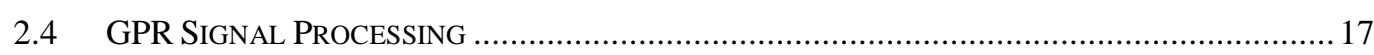

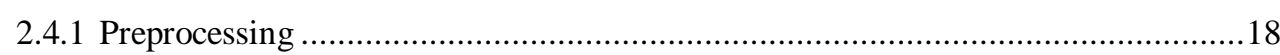

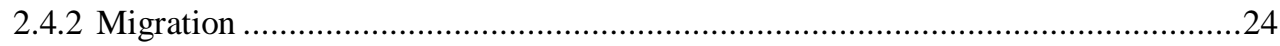

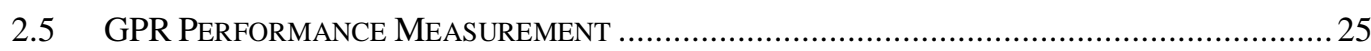

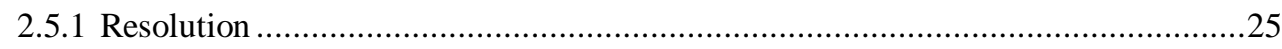

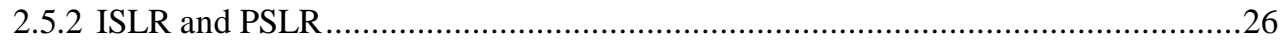

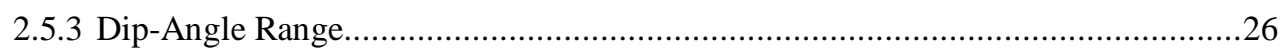

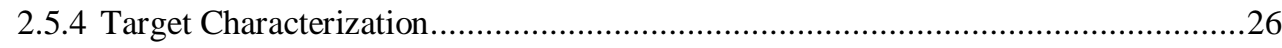

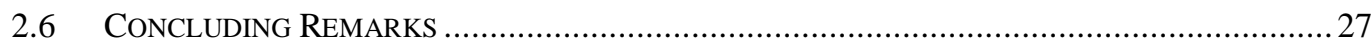

CHAPTER 3 THE SPATIAL DIVERSITY FOR UWB GPR SYSTEMS.....................................28

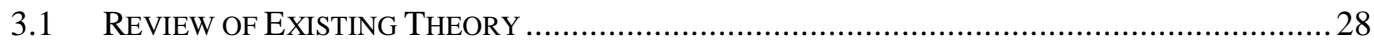

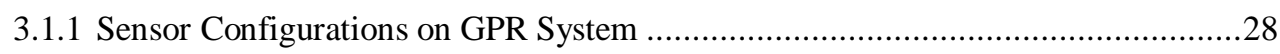

3.1.2 Migration Formula of SISO, SIMO, and MIMO Configurations ...........................29

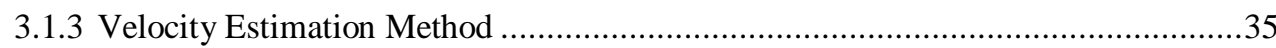


3.2 PERFORMANCE ANALYSIS OF MIMO CONFIGURATION FOR UWB GPR ......................... 36

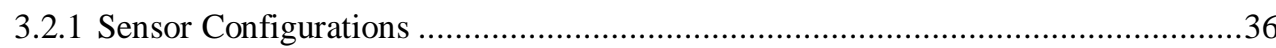

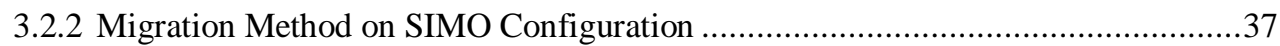

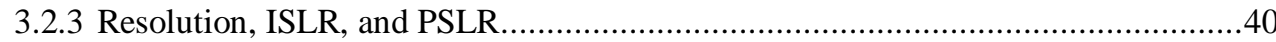

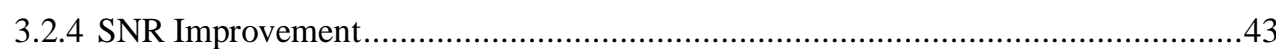

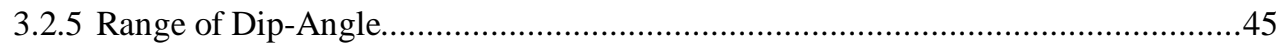

3.2.6 Theoretical Analysis of Array Performance ..................................................48

3.3 COMPARISON OF Migration Methods ..................................................................... 57

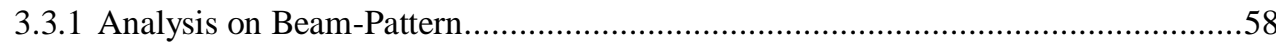

3.3.2 Analysis on Distributed Targets .................................................................60

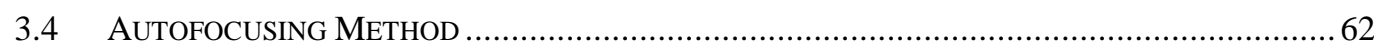

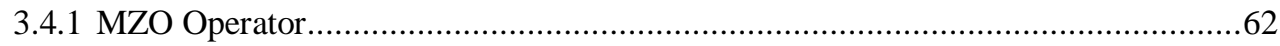

3.4.2 Proposed Autofocusing Method ....................................................................64

3.4.3 Results and Performance Analysis .............................................................66

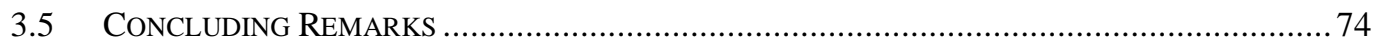

CHAPTER 4 THE WAVEFORM DIVERSITY FOR UWB GPR SYSTEMS............................76

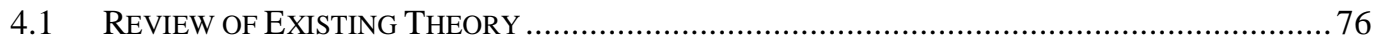

4.1.1 Waveform Diversity in Radar System ....................................................... 76

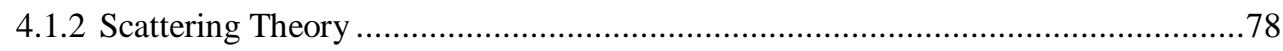

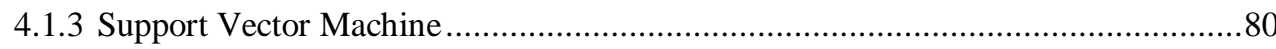

4.2 Proposed WAVEFORM DIVERSITY METHOD FOR UWB GPR …................................. 83

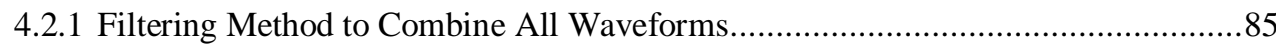

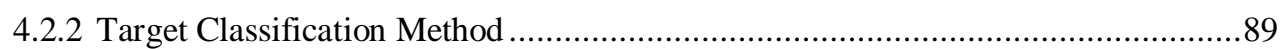

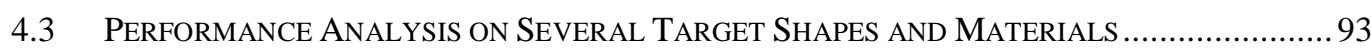

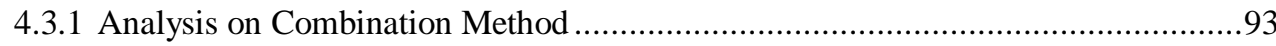

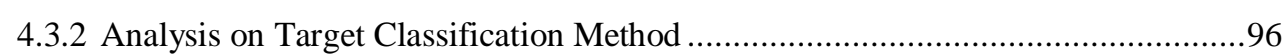

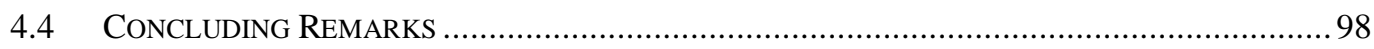

CHAPTER 5 THE POLARIZATION DIVERSITY FOR UWB GPR SYSTEMS .....................99

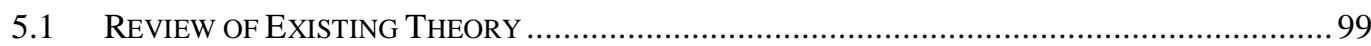

5.1.1 Polarimetric in Synthetic Aperture Radar System ...........................................99

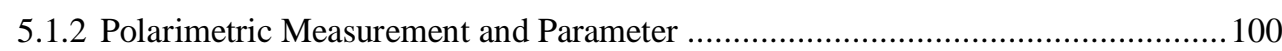

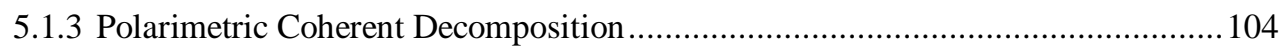

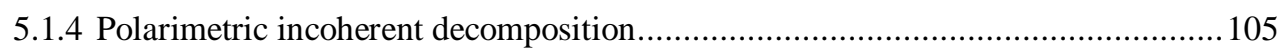

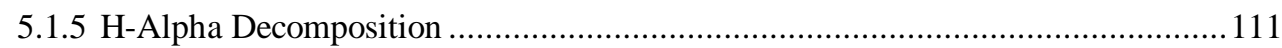

5.2 Proposed Polarization Diversity Method For UWB GPR .............................. 113

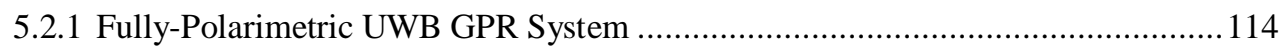

5.2.2 Implementation of PolSAR Decomposition Method on UWB GPR Data ............115

5.3 PERFormance of Polarimetric UWB GPR Methods on SimUlation Data............. 116 
5.3.1 Response of Decomposition Methods on Variation of Target Shapes ..................116

5.3.2 Response of Decomposition Methods on Variation of Target Size ...................... 120

5.4 PERFormance of Polarimetric UWB GPR Methods on EXPERIMENTAL DatA ........ 123

5.5 COMPARISON OF POLARIMETRIC CHARACTERISTIC OF SISO AND MIMO CONFIGURATIONS..

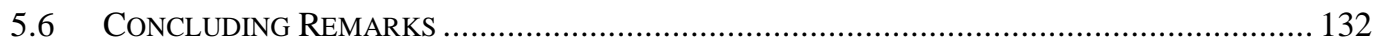

CHAPTER 6 CONCLUSIONS AND FUTURE WORK.......................................................133

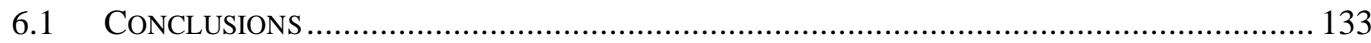

6.1.1 Analysis on SISO, SIMO, and MIMO Imaging Performance ............................. 134

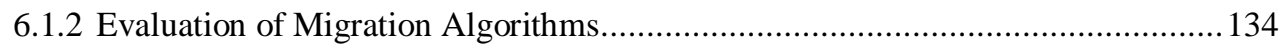

6.1.3 Efficient Autofocusing Method for MIMO Configuration.................................135

6.1.4 Implementation of Waveform Diversity on UWB GPR ................................ 135

6.1.5 Evaluation of Polarimetric Decomposition Methods on UWB GPR for Utilities

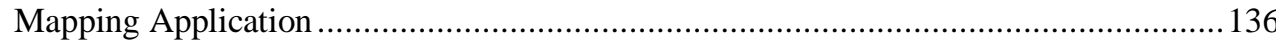

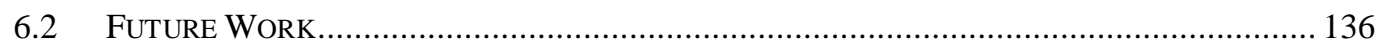

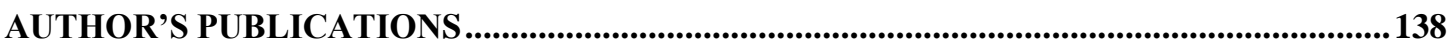

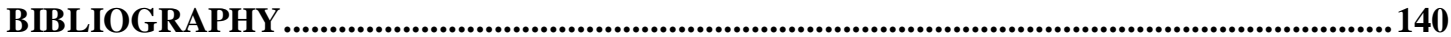




\section{LIST OF FIGURES}

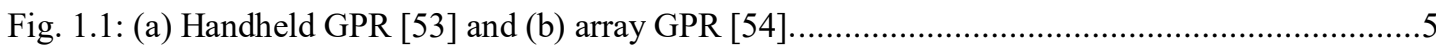

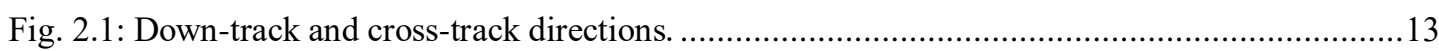

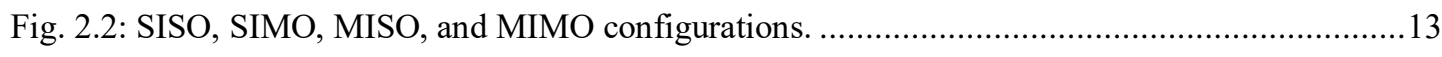

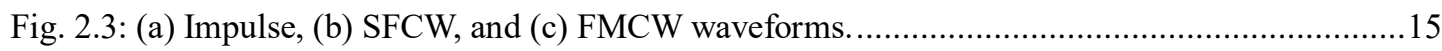

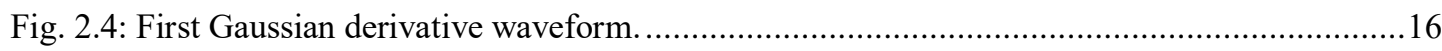

Fig. 2.5: Hermite polynomial waveforms in (a) time domain and (b) frequency domain......................16

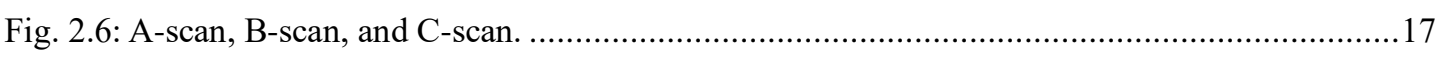

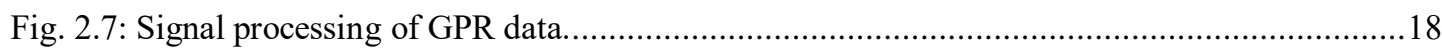

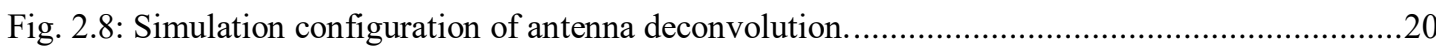

Fig. 2.9: Simulation result of (a) received signal and (b) deconvolution.............................................20

Fig. 2.10: Target configuration in the simulation for data preprocessing. .........................................21

Fig. 2.11: (a) Simulation result and (b) ground-bounce localization result.......................................21

Fig. 2.12: Result of background removal by using (a) mean-based estimator and (b) PCA.................23

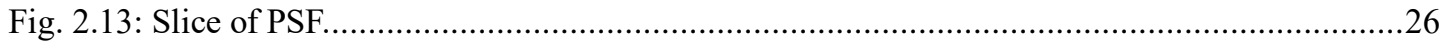

Fig. 3.1: GPR array configuration and movement direction............................................................22

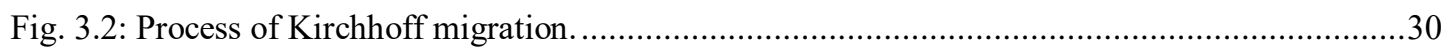

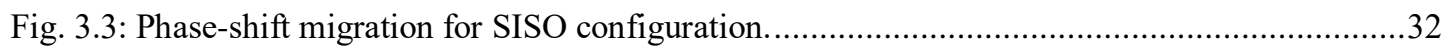

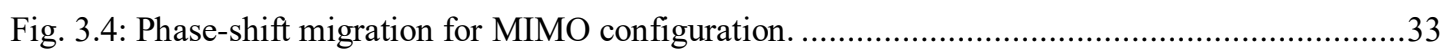

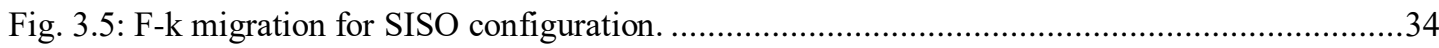

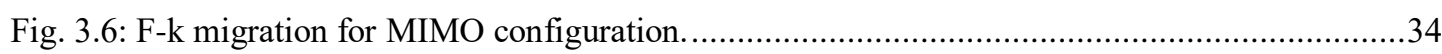

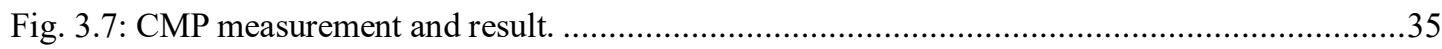

Fig. 3.8: SISO, SIMO, MIMO-half, and MIMO-all array configurations...........................................37

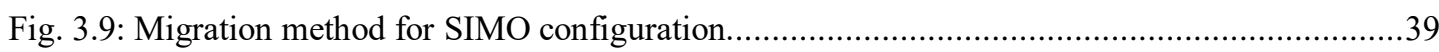

Fig. 3.10: Results of Kirchhoff migration and the proposed migration method on point target.............39

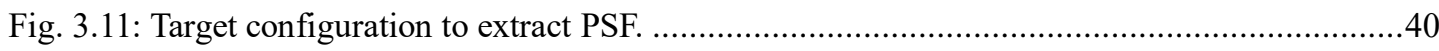

Fig. 3.12: Point spread function of the 4 array configurations........................................................... 41

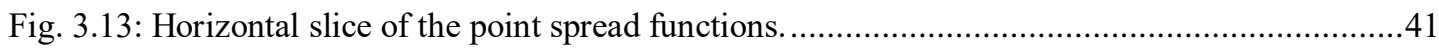

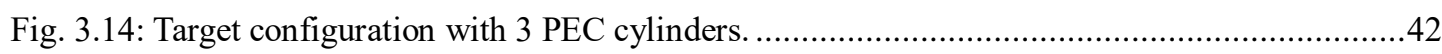

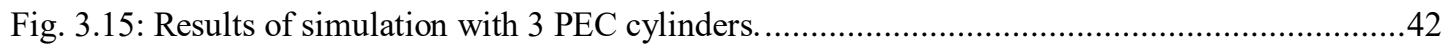

Fig. 3.16: Target configuration with thin void. ….....................................................................4

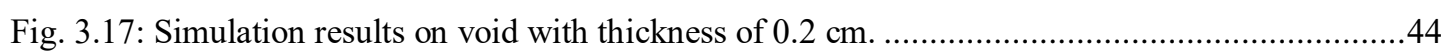

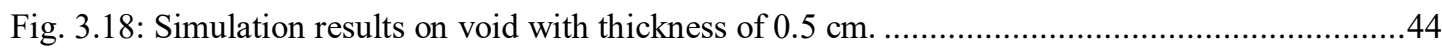

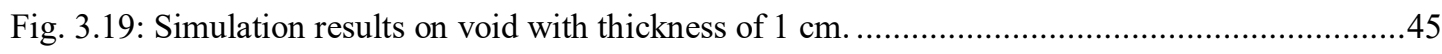

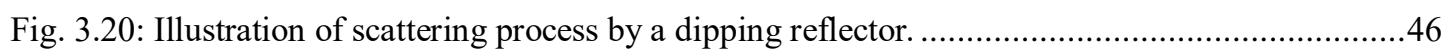

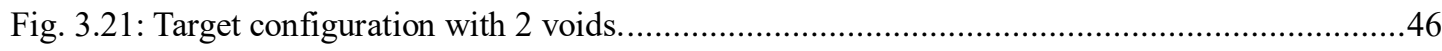

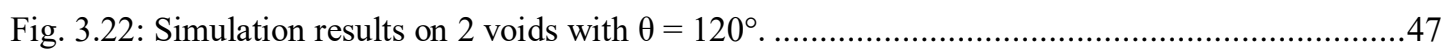

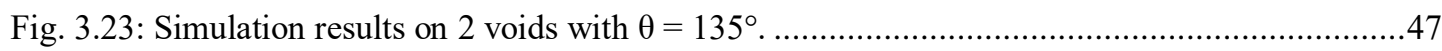


Fig. 3.24: Simulation results on 2 voids with $\theta=150^{\circ}$

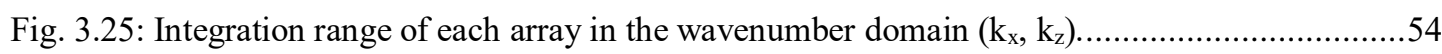

Fig. 3.26: Normal vectors that can be resolved by each array..............................................57

Fig. 3.27: Beam-patterns by using summation over depth dimension of the PSF...........................59

Fig. 3.28: Beam-patterns by taking horizontal slice at the peak of the PSF....................................60

Fig. 3.29: Simulation configuration for analysis on distributed targets......................................60

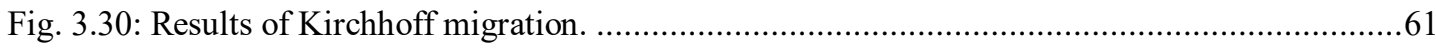

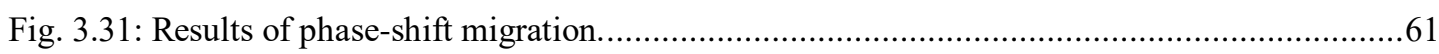

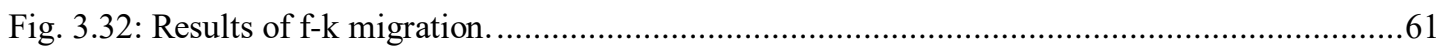

Fig. 3.33: F-k migration steps for MIMO configuration. ..............................................................63

Fig. 3.34: Migration on a point target with different wave velocities. .......................................65

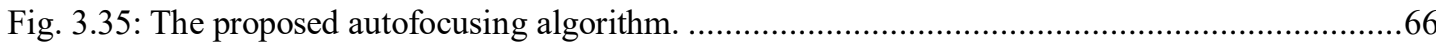

Fig. 3.36: Standard autofocusing on SISO configuration. ...........................................................66

Fig. 3.37: Standard autofocusing on MIMO configuration. ...................................................66

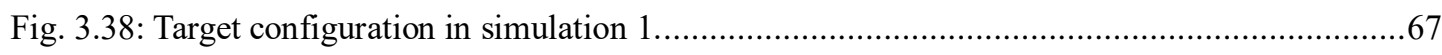

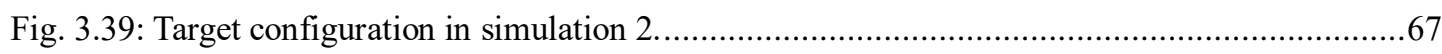

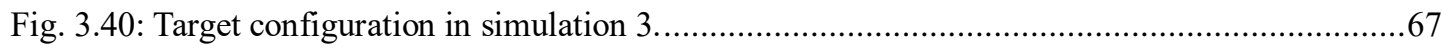

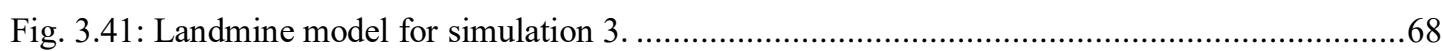

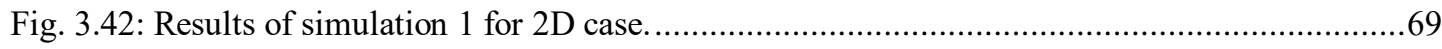

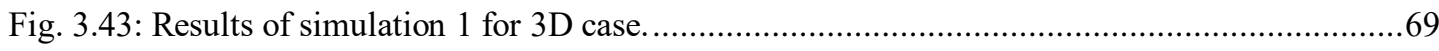

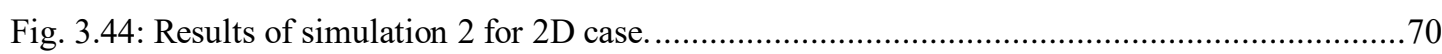

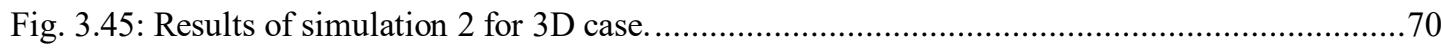

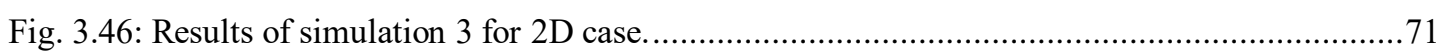

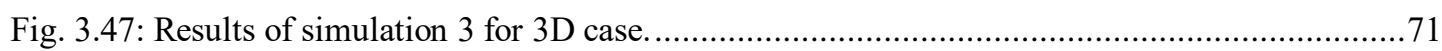

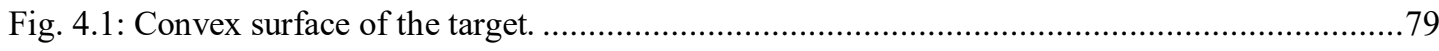

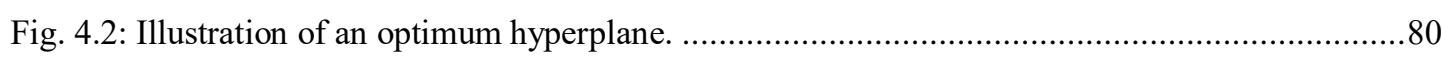

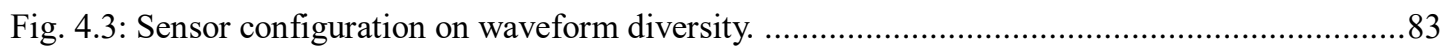

Fig. 4.4: Original Hermite polynomial waveforms and spectrums. ........................................8

Fig. 4.5: Hemite polynomial waveforms and spectrums in GPRMax simulation. ............................8 84

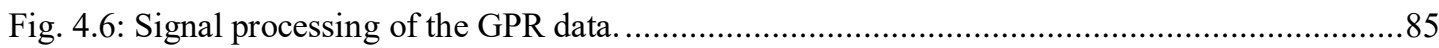

Fig. 4.7: Diagram of the filtering method to combine all waveforms. .............................................86

Fig. 4.8: Effect of several measurement factors on integration range in wavenumber domain.............88

Fig. 4.9: Integration range of each waveform in wavenumber domain. .................................... 88

Fig. 4.10: Simulation configurations on several target materials................................................... 90

Fig. 4.11: Combination of amplitude ratios of $1^{\text {st }}, 2^{\text {nd }}$, and $3^{\text {rd }}$ order HP over $0^{\text {th }}$ order HP................90

Fig. 4.12: Diagram of the classification method..........................................................92

Fig. 4.13: Horizontal beam-patterns of HP waveforms and their combination..................................94

Fig. 4.14: Vertical beam-patterns of HP waveforms and their combination. ...................................94

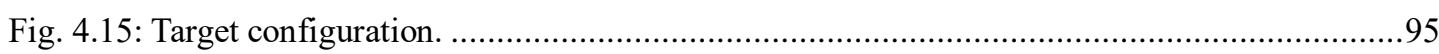




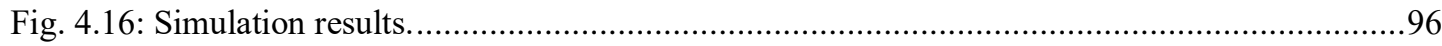

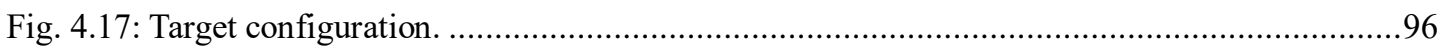

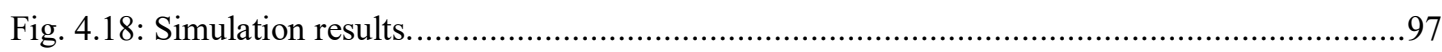

Fig. 4.19: Target classification results with green line for class 1 and red line for class 2 ..................98

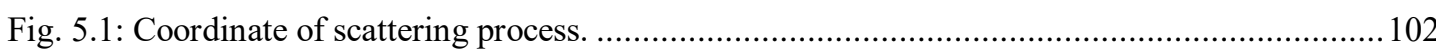

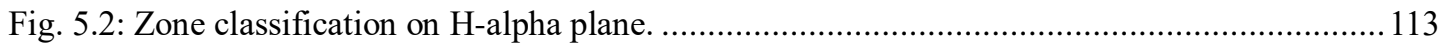

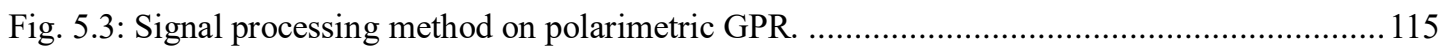

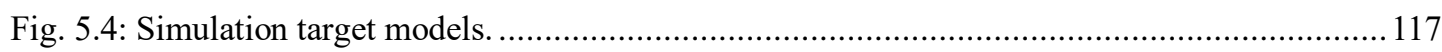

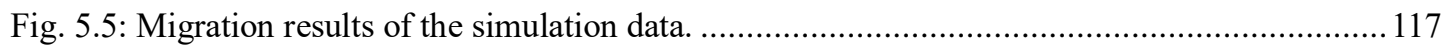

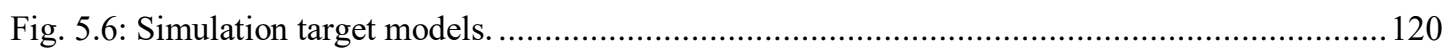

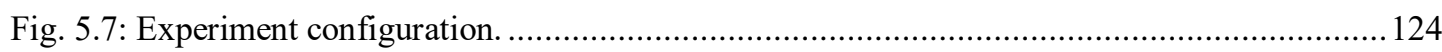

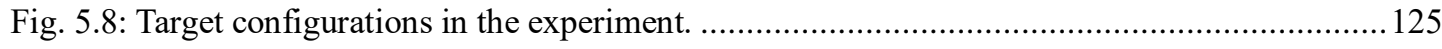

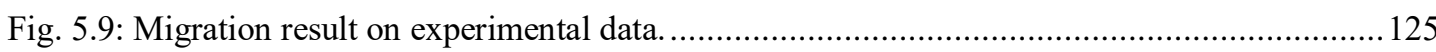

Fig. 5.10: Target models for simulation of polarimetric characteristic in MIMO configuration.........128

Fig. 5.11: Results of Pauli decomposition on SISO and MIMO configurations. .............................130

Fig. 5.12: Results of H-alpha decomposition on SISO and MIMO configurations............................132 


\section{LIST OF TABLES}

Table 3.1: Performance Parameters of Each Sensor Configuration..................................................41

Table 3.2: Summary of Performance of Each Migration Algorithm .............................................62

Table 3.3: Average Computation Time of the Autofocusing Methods.............................................72

Table 3.4: Computational Complexity of Standard SISO Autofocusing ........................................72

Table 3.5: Computational Complexity of Standard MIMO Autofocusing ........................................73

Table 3.6: Computational Complexity of the Proposed MIMO Autofocusing.................................73

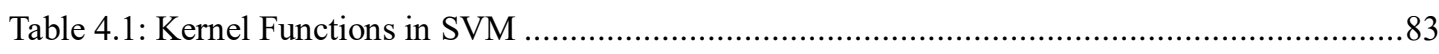

Table 4.2: Percentage of Classification Errors............................................................................ 92

Table 4.3: Resolution of Each Waveform and the Combinaton Method .........................................95

Table 5.1: Results of Polarimetric Decomposition Methods on Various Target Shapes .................... 119

Table 5.2: Results of Polarimetric Decomposition Methods on Various Target Sizes ......................121

Table 5.3: Results of Polarimetric Decomposition Methods on Experimental Data ........................ 126 


\section{LIST OF NOTATIONS}

$x$

$\mathbf{X}$

$\mathbf{X}$

$(\bullet)^{T} \quad$ Transpose operation

$(\bullet)^{H} \quad$ Conjugate transpose operation

Scalar variable

Column vector

Square or rectangular matrix 


\section{LIST OF ABBREVIATIONS}

1D

2D

3D

AWGN

CMP

ERM

FDTD

FFT

FMCW

GPR

HP

IFFT

ISLR

MIMO

MISO

MOSP

$\mathrm{MZO}$

PCA

PEC

PO

PolSAR

PSF

PSLR

SAR

SFCW

SIMO

SISO

SNR

SVM

UWB

UXO

VNA
One-Dimensional

Two-Dimensional

Three-Dimensional

Additive White Gaussian Noise

Common Mid-Point

Exploding Reflector Model

Finite-Difference Time-Domain

Fast Fourier Transform

Frequency Modulated Continuous Wave

Ground Penetrating Radar

Hermite Polynomial

Inverse Fast Fourier Transform

Integrated Side-Lobe Ratio

Multi-Input Multi-Output

Multi-Input Single-Output

Method of Stationary Phase

Migration to Zero-Offset

Principal Component Analysis

Perfect Electric Conductor

Physical-Optic

Polarimetric Synthetic Aperture Radar

Point Spread Function

Peak Side-Lobe Ratio

Synthetic Aperture Radar

Stepped-Frequency Continuous Wave

Single-Input Multi-Output

Single-Input Single-Output

Signal-to-Noise Ratio

Support Vector Machine

Ultra-Wideband

Unexploded Ordnance

Vector Network Analyzer 


\section{CHAPTER 1}

\section{Introduction}

For underground geological feature survey, current approaches include mechanical probe drilling approach, seismic tomography, and ground penetrating radar (GPR). Mechanical probe drilling approach can provide direct assessment of the geological conditions at the probe-hole locations, but it does not provide sufficient information beyond the probe-hole locations, and it is an expensive, time-consuming and destructive technique. The seismic method, typically utilizing the stress wave reflection from an explosive source, can cover a large area with deep penetration but it is difficult to image slender structures due to its poor resolution and not suitable for fine geological feature detection [1], [2]. GPR has the advantage of providing non-destructive survey and is the desirable means for underground remote sensing. Current GPR technologies have been used successfully in certain applications such as oil/gas/mine resources survey, utility (pipeline) detection, and soil/rock interface identification, but they are not capable to detect small features, such as joints/faults/gaps of a few millimeter thickness and arbitrary faces. In this project, all possible resources will be studied and explored to improve the performance of GPR. In this chapter, the basic concept of GPR and possible resources that would help to improve GPR performance will be introduced. The motivation, objectives, and the challenges of the research will be described. The major contribution of this study will be highlighted. At the end, the organization of the thesis will be explained.

\subsection{Overview of Ultra-Wideband Ground Penetrating Radar System}

Technologies that enable us to observe subsurface objects have become demands over broad subjects from military to commercial needs in which a tool for detecting, locating, and identifying subsurface target is highly desired. Several methods, such as acoustic sensor, seismic imaging, electromagnetic induction detector, optical detector, and etc. have been developed to fulfill such needs. Among them, GPR becomes one of the attractive solutions due to its high resolution and non-destructive properties. 
GPR is a subsurface imaging system that utilizes electromagnetic wave in microwave frequency domain. Conventional GPR commonly operates in frequency range from $500 \mathrm{MHz}$ to $5 \mathrm{GHz}$. This frequency range provides sufficient penetration of electromagnetic wave through ground while preserving enough resolution to observe subsurface targets. Higher operational frequencies are also available in several GPR systems to achieve higher resolution for shallow buried objects.

GPR system has been used for various applications in the following fields.

\section{1) Civil Engineering}

Due to its high resolution and efficient measurement process, GPR becomes a preferred tool in several civil engineering applications. Some GPR systems have been developed for utilities mapping [3], [4], that is, to obtain the location and image of underground infrastructure. GPR is also used for inspection of infrastructure such as pavement [5], road [6], [7], bridge [8], [9], airfield runway [10], rail [11], building [12], [13], etc. This inspection is necessary to maintain the condition of such structures and to avoid fatal accident.

\section{2) Archaeological}

In archaeological survey, GPR is used to observe structure and objects inside historical building or site. For instances, in [14] a handheld GPR was used to observe the reinforcement structure in historical site of Basilica of Sant'Andrea in Mantua, while in [15], GPR was used to identify and map pavement construction periods in silo-yard of Valencia. Another work in [16] utilized GPR to detect buried historical site in Sendai of Northeast Japan.

3) Military

The most common application of GPR in military is for landmine and UXO detection purpose [17], [18]. Landmines and UXOs have remained to be threats in many countries even long after war period. It was estimated that around 60 countries are still contaminated by landmines [19]. Some GPR systems in various types have been proposed to resolve this issue. In [20], a combination of handheld GPR and metal detector was developed. Another work in [21] utilized single-input multiple-output 
(SIMO) array GPR to improve measurement speed in landmine detection. A fullypolarimetric GPR was proposed in [22] along with landmine detection algorithm based on polarization and frequency response.

\section{4) Geophysics}

In geophysics, GPR is used to extract soil parameters, such as soil water content [23][28], soil dielectric properties [29]-[31], and soil hydraulic parameters [32], [33]. Moreover, GPR has also been used to observe the condition of several geophysical structures such as glacier [34], [35], sand dunes [36], and volcano [37].

The focus of this thesis is on GPR system that complies with the UWB requirement. According to the definition from Defense Advanced Research Project Agency (DARPA) [38], UWB is defined as a system with fractional bandwidth $(\% B W)$ greater than $25 \%$, where the fractional bandwidth is given in (1.1).

$$
\% B W=\frac{2\left(f_{H}-f_{L}\right)}{f_{H}+f_{L}}
$$

UWB concept has been developed for radar [39], [40], imaging [21], [41], positioning [42], and communication system [43]. Since Federal Communications Commission (FCC) released new bandwidth regulation $(3.6-10.1 \mathrm{GHz})$ for UWB application in 2002 , there is a growth in research on UWB communication system [44]-[47]. UWB was developed for wireless personal area network and body area network, where short-range and high data rate systems are highly demanded. However, due to the failure in achieving IEEE standardization and abandonment of the technological investment by industry [48], the development of UWB technology for communication system has been stalled and decreased, and the UWB research trend tends to focus on radar sensor technology.

In radar system such as through-wall radar, imaging radar, and positioning system, the UWB properties give advantages in high resolution, high susceptibility to noise, and low probability of interception. Meanwhile, the implementation of UWB technology for GPR system gives advantages mainly by its low center frequency and high bandwidth properties. The low center frequency is necessary since the subsurface attenuation becomes significant in high frequency range. Moreover, the high bandwidth is useful to obtain high resolution image of underground target. 


\subsection{Diversities in Ultra-Wideband Ground Penetrating Radar}

GPR systems that are available in the market come up with numerous variations in antenna system, number of channel, sensor configuration, operating frequency, transmitted waveform, polarization, etc. Due to such flexibility in hardware implementation, the signal processing methods are also adjusted to deal with the corresponding system configuration. Some GPR systems are developed with particular signal processing method that can perform detection or analysis on certain target, such as landmine and UXO [17], [22], defect in structure [12], [49]-[52], soil water content [23]-[28], etc. Such variation enables the implementation of diversity concept on GPR system.

In communication system, diversity is defined as method to improve the reliability of system by using two or more communication channels with different characteristics. This concept has been generalized into radar system, where some measurement channels with different characteristics are used to improve the target detection and characterization performance. This research considers mainly 3 diversities in GPR system as follows:

1) Spatial Diversity

This diversity can be achieved by modifying the configuration of transmitters and receivers. GPR system can be implemented with several sensor configurations. The simplest one only has a pair of transmitter and receiver as shown in Fig. 1.1(a). To obtain the image of subsurface, this sensor must be moved point-by-point to collect the data. This method might be acceptable for small scale application, but will be troublesome for wide area survey.

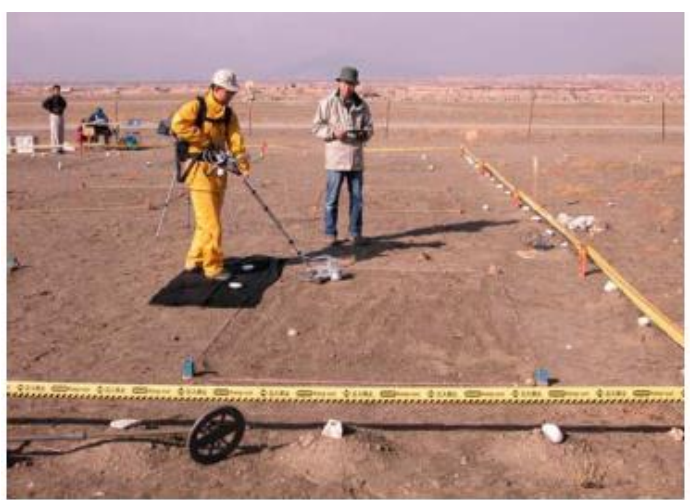

(a)

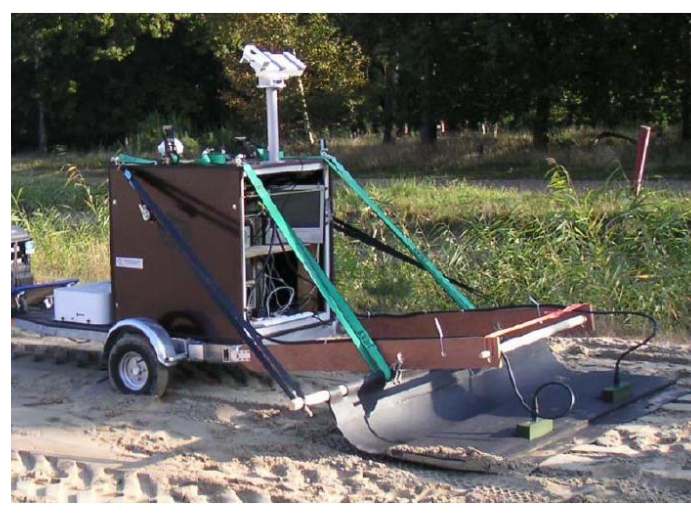

(b)

Fig. 1.1: (a) Handheld GPR [53] and (b) array GPR [54]. 
In order to improve the measurement speed, some GPR system utilizes array of sensor as shown in Fig. 1.1(b). There are several types of array that have been proposed. In many commercial GPR systems, the array comprises several pairs of transmitter and receiver in linear arrangement [10]. Another array configuration proposed in [21] consists of only one transmitter and several receivers in which the data collection process only involves single transmission and simultaneous reception. This configuration was used for landmine detection and was shown to have comparable performance with a single-pair measurement while providing better efficiency in measurement speed and number of component.

In recent research on GPR, a more flexible array which is well-known as multi-input multi-output (MIMO) or multi-static configuration was proposed [16], [55]-[57]. In this configuration, the array comprises several transmitters and receivers that work together. MIMO configuration was originated in communication system and has been adapted into radar system. Some works in narrow-band radar system [58], [59] show that MIMO can improve the number of target that can be identified, allow direct applicability of adaptive technique, and improve the flexibility of transmit beampattern design. Other research in UWB imaging radar [60], [61] show that MIMO configuration can reduce the number of transmitters and receivers while maintaining the same performance to synthetic aperture array. In [55] and [56], an array that consists of 2 transmitters and several receivers was proposed for GPR system and it was shown that such configuration can reduce the clutter and improve target detection performance. Another work in [16] allows all transmitters and receivers to work together in collecting data on archaeological site and demonstrates that this configuration can improve the imaging capability.

\section{2) Waveform Diversity}

Waveform diversity is an old technique that has been employed in communication [62], [63] and radar system. In MIMO narrow-band radar system, waveform diversity is used to obtain more channels, in which some transmitters emit different waveform with particular coding scheme [59]. Another application of waveform diversity is on radar for tracking purpose [64], [65]. This technique was inspired from bat behavior when tracking its prey in which such animal uses waveform diversity with frequency modulated waveform. In imaging radar, utilization of waveform diversity for synthetic 
aperture radar (SAR) system was proposed in [66]-[68]. Coding scheme was applied to chirp rate of frequency-modulated continuous wave waveform to obtain orthogonal transmission channel on SAR system. Although waveform diversity has been developed for communication and radar system, to the author knowledge, there are only few attempts on implementation of waveform diversity in GPR system. One of them is given in [69], where 2 impulse waveforms with different pulse width were used for landmine detection on GPR.

As elaborated previously, in this study the transmitted waveform of the GPR system must satisfy UWB criteria. There are 3 commonly used waveforms in GPR system: impulse waveform, stepped-frequency continuous wave (SFCW), and frequencymodulated continuous wave (FMCW). In this research, only impulse waveform is used for study of waveform diversity.

In [70], a set of Hermite polynomial waveform was used for waveform diversity on UWB communication system. Due to some similarities between UWB communication system and UWB radar system, Hermite polynomial waveform set is selected to implement waveform diversity in this study.

\section{3) Polarization Diversity}

Another important aspect of GPR system is the antenna polarization. Linear polarization and circular polarization are the most common polarizations for GPR antenna. However, linear polarization is preferable over circular polarization since it is difficult to maintain the antenna in circular polarization for high bandwidth system. Normally, GPR antenna has linear polarization only at one direction, either alongtrack or cross-track direction. Nevertheless, recent research on GPR system [22], [52], [71]-[75] has moved towards the utilization of partially- or fully-polarimetric data in which the measurement is conducted in combination of 2 orthogonal polarization bases.

Polarimetric SAR (PolSAR) system is a famous example of polarization diversity in radar system. In this method, the data is collected in partially- or fully-polarimetric mode, and several signal processing methods have been developed to combine such data and perform analysis on target characterization and classification. Recently, a number of works have applied these PolSAR signal processing techniques into 
polarimetric GPR. However, since PolSAR methods have been developed over decades, there still exists many methods that have not been adapted yet into GPR system, and this thesis will focus on exploration of such methods.

\subsection{Motivations}

Singapore government has planned to invest in creating and using underground space for future growth [76]-[78]. The underground space development has also unlimited potential around the world [78]. One of the main challenges in underground space construction is the uncertain and complex geological and hydro-geological conditions ahead of cavern/tunnel face. Though Singapore is small, it contains rapidly changing geology (various rock formations) and geological features (joints, faults, water bearing zone, weak layers, cavities, and etc.). The huge uncertainties associated with unknown local geological conditions have substantially increased the risk for underground construction, subsequently lead to increase in construction cost and duration.

Based on the demand for higher resolution GPR imaging from industries, the following factors motivate this research:

a) A good imaging capability is highly desired in many GPR applications. GPR system is expected to have high resolution, low side-lobes, high dip-angle range, and produces less image artifact. In addition, data acquisition speed is crucial in some applications. A conventional GPR system that utilizes only a pair of sensor with single waveform and polarization might not be sufficient to satisfy all these requirements. Meanwhile, some elements of GPR system, such as antenna array configuration, transmitted waveform, and polarizations, can be implemented and modified in numerous ways, which give possibility to improve the imaging performance.

b) Data interpretation still becomes a challenge in GPR measurement. Due to limitation in resolution, clutter, hyperbolic diffraction, and subsurface inhomogeneity, target shape will be distorted in GPR image and the interpretation of such data requires a trained user. Moreover, conventional measurement method does not provide adequate information to distinguish several target shapes and materials.

c) Implementation of diversity on the measurement configuration can potentially improve the imaging performance and data interpretation. However, such 
modification will affect the signal processing side, since some processing techniques for conventional configuration are not suitable to handle the modified GPR configuration. Therefore, new processing methods need to be developed to deal with the system configuration and to process additional information provided by the diversity.

By considering all the issues that have been described, a study on GPR configuration that incorporates spatial, waveform, and polarization diversities to improve the imaging capability and data interpretation is necessary in general. In particular, we focus on the investigation of performance improvement of each diversity and the development of advanced signal processing methods to exploit additional information in the modified GPR measurement.

\subsection{Research Objectives}

As described in the previous subsection, the primary focus of this thesis is on the investigation of potential performance improvement provided by spatial, waveform, and polarization diversities in GPR system. Specifically, the main research objectives in this thesis are as follow:

1) To perform comprehensive study on the implementation of spatial, waveform, and polarization diversities in UWB GPR. The performance improvement and drawbacks are explored and compared with conventional GPR configuration.

2) To develop signal processing methods that compatible with the modified sensor configuration of UWB GPR. In particular, modification on migration and velocity estimation method are necessary to handle various array configurations.

3) To develop signal processing method that can utilize the waveform diversity data to improve target interpretation. The performances of this method are evaluated on several target shapes and material.

4) To modify and apply various PolSAR signal processing methods for fullypolarimetric GPR system. The performance of each method is analyzed on various target shapes and materials with focus on utility mapping application. 


\subsection{Major Contributions}

This thesis provides the following novelties and contributions to the corresponding research field:

1) A study on several linear array configurations of UWB GPR has been performed. This study includes analysis of array performance based on several parameters, migration methods, and applications. The performance of SISO, SIMO, and MIMO linear array configurations has been formulated in terms of resolution, SNR, and range of dip-angle. Based on the analytical formulation, it can be shown that MIMO configuration provides performance improvement in the corresponding aspects. The result has also been verified with simulation data on the three array configurations.

2) A new migration method to handle SIMO configuration has been formulated. To the author knowledge, data processing on SIMO configuration employs Kirchhoff migration for the focusing method. The proposed migration method is a modification of $\mathrm{f}-\mathrm{k}$ migration algorithm. Thus, it maintains the fast computation properties of $\mathrm{f}-\mathrm{k}$ migration with comparable image quality to Kirchhoff migration.

3) A novel autofocusing method has been proposed to handle MIMO data efficiently. Information of wave velocity in the subsurface is necessary to obtain focused image. However, in most of real cases, such parameter is unknown and needs to be estimated from the data. Autofocusing algorithm can overcome this velocity estimation issue. However, due to the large data size in MIMO configuration, direct autofocusing on MIMO data will produce high computation time especially on 3D data. The proposed autofocusing method can handle MIMO data efficiently by transforming the data into equivalent SISO data, and thus reducing the computation time significantly.

4) Implementation of waveform diversity on UWB GPR has been proposed. In this method, several impulse waveforms from Hermite polynomial set are used to collect the GPR data independently. Each waveform occupies different frequency band, and thus can provide different information regarding the target.

5) An algorithm to process waveform diversity data and to improve target interpretation has been developed. This algorithm combines the GPR data from several waveforms, extracts necessary features, and processes these features to obtain more information regarding the target material. The results demonstrate the advantages of waveform 
diversity in discriminate target made of free-space, water, and perfect electric conductor.

6) Study of PolSAR processing method on UWB GPR data has been conducted. Some prior works on GPR system have applied PolSAR processing methods on fullypolarimetric GPR data. This thesis performs further exploration on PolSAR methods with focus on utilities mapping application.

\subsection{Thesis Organization}

This thesis is organized as follows.

Chapter 1 introduces the basic concept of ground penetrating radar (GPR) along with the challenges and possible research directions to improve GPR performance. Besides, the research motivation, objectives, and major contributions of this thesis are explained.

Chapter 2 provides introduction to UWB GPR technology. Some hardware aspects such as measurement process, sensor configuration, operating frequency, waveform, and polarization are described. The fundamental of GPR signal processing methods are elaborated along with several parameters to measure the imaging performance.

Chapter 3 presents the study of spatial diversity for UWB GPR. A deeper theoretical concept regarding sensors configuration, migration algorithms, and velocity estimation method are first provided. Then, the proposed MIMO configuration is described. Performance evaluation of SISO, SIMO, and MIMO configurations is given on various target configuration and GPR applications. Finally, the proposed autofocusing method for MIMO configuration is explained along with the corresponding simulation result and performance analysis.

Chapter 4 investigates the implementation of waveform diversity on UWB GPR. Theoretical concept of waveform diversity in UWB communication system, generalization to radar system, and scattering theory are first provided. Then, the implementation of waveform diversity on UWB GPR is given, followed by the signal processing methods. Finally, the performance study on several target shapes and materials is presented.

Chapter 5 presents the implementation and analysis of polarization diversity on UWB GPR. Review of PolSAR theory is first given. Then, the implementations of polarization diversity method and PolSAR techniques for UWB GPR are provided. Finally, result and performance 
of several PolSAR methods on both simulation and experimental fully-polarimetric GPR data are investigated.

Chapter 6 summarizes all the works provided in this thesis and provides recommendations of future work. 


\section{CHAPTER 2}

\section{Fundamentals of Ultra-Wideband Ground Penetrating}

\section{Radar}

Ground penetrating radar (GPR) is a subsurface imaging method that utilizes electromagnetic wave in microwave frequency domain. This thesis focus on GPR system that satisfies ultrawideband (UWB) requirement, i.e., fractional bandwidth of the system must be greater than $25 \%$ as described in Section 1.1. UWB GPR has been used for numerous applications in several fields of study, such as non-destructive testing and utilities mapping in civil engineering [3]- [13], inspection of historical site in archaeology [14]- [16], landmine and UXO detections in military [17]- [22], study of soil structure in geophysics [23]-[33], etc.

In developing UWB GPR system, several aspects can be modified to adjust the imaging performance on particular application. Some of the essential parameters are sensor configuration, center frequency, waveform, and antenna polarization. In the following sections, all these parameters are described.

\subsection{GPR Sensor Configurations}

There are several sensor configurations that have been used in the existing GPR system. The most basic configuration comprises a single pair of transmitter and receiver that moves over the entire scan area to collect the data point-by-point. Despite of its simplicity, the data collection process of such configuration becomes very time-consuming on large area.

In order to improve the data acquisition speed, arrays of sensor are employed in some GPR systems. To obtain 3D data, this array only needs to move in one direction over the scan area, which is so-called as down-track direction. Meanwhile, its orthogonal direction in which the sensors are elongated is well-known as cross-track direction. These directions are illustrated in Fig. 2.1. 


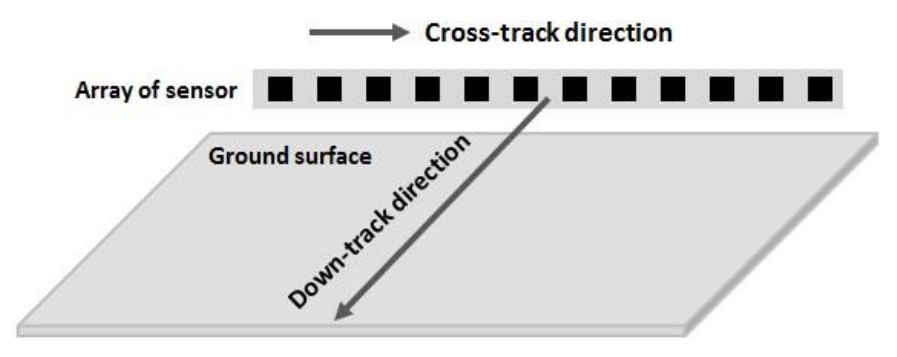

Fig. 2.1: Down-track and cross-track directions.

In configuring a linear array, there is flexibility in choosing the number of sensor elements and the corresponding transmit and receive mechanism. The most typical linear array configuration is single-input single-output (SISO), where several pairs of transmitters and receivers work independently and collect the data sequentially at different time instance [79]. Another configuration is single-input multi-output (SIMO), as proposed in [21]. This configuration employs one transmitter and an array of receiver. SIMO configuration gives advantages of fast measurement speed with less number of components compared to SISO array. In [21], SIMO configuration was tested for landmine detection application, and it exhibits improvement in the data acquisition speed with simplification in antennas and electronics system. An inverted version of such configuration is multi-input single-output (MISO), which consists of several transmitters and one receiver. Although MISO configuration is not common in GPR system, it has been used in some radar and seismic imaging systems.

Recently, progresses have been made on study of more flexible configuration namely multiinput multi-output (MIMO). In contrast to SISO configuration, MIMO configuration allows any transmitter and receiver in the array to work together simultaneously. Thus, MIMO array enables higher point of view compared to SISO and SIMO arrays at the expense of larger amount of data and complexity. Fig. 2.2 illustrates all the configurations that have been described so far.
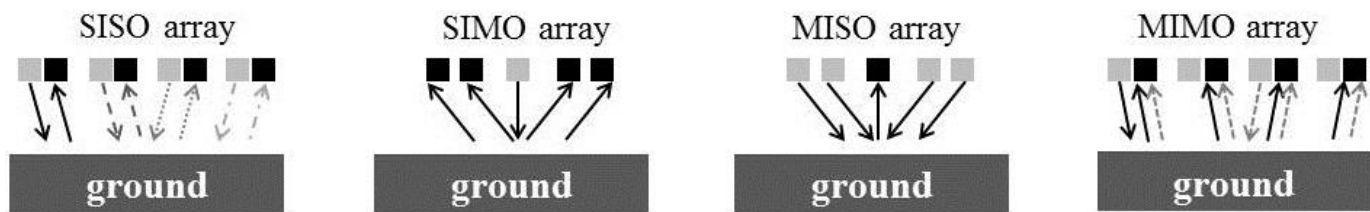

Transmitter
Receiver

Fig. 2.2: SISO, SIMO, MISO, and MIMO configurations. 
Some prior works on GPR system have shown the advantages of MIMO configuration compared to the conventional sensor configuration. In [60] and [61], it was demonstrated that MIMO configuration with 2 transmitters and several receivers can reduce the clutter and improve the target detection capability. In [16], a multi-static GPR array was used to collect data in archaeological site. The multi-static array allows all transmitters and receivers to work together and it was presented that such configuration can improve the imaging performance. These results indicate that MIMO configuration is suitable to implement the spatial diversity concept. However, some issues must be considered before practically apply MIMO configuration. Firstly, MIMO configuration yields higher complexity in hardware and measurement process. Although the complexity can be reduced by utilizing smaller number of transmitters or receivers, the imaging performance will also degrade. Thus, it is necessary to obtain analytical formulation of MIMO array performance in order to control the trade-off between array complexity and imaging performance. Secondly, the signal processing algorithms must be modified to deal with such configuration. All these issues will be addressed in Chapter 3.

\subsection{GPR Frequency and Waveform}

As explained in Section 1.1, we focus on GPR system that utilizes UWB waveform. We recall that the fractional bandwidth of UWB waveform $(\% B W)$ must satisfy $(2.1)$, where $f_{H}$ is the upper boundary of the frequency spectrum, $f_{L}$ is the lower boundary of the frequency spectrum, and $f_{C}$ is the center frequency. Since the shape of the spectrum is not strictly specified in (2.1), there can be numerous UWB waveform shapes with the same operating center frequency.

$$
\% B W=\frac{f_{H}-f_{L}}{f_{C}} \geq 25 \%
$$

There are 3 waveforms that are mainly used for UWB GPR system: impulse waveform, stepped frequency continuous wave (SFCW), and frequency-modulated continuous wave (FMCW). Fig. 2.3 illustrates these three waveforms. 


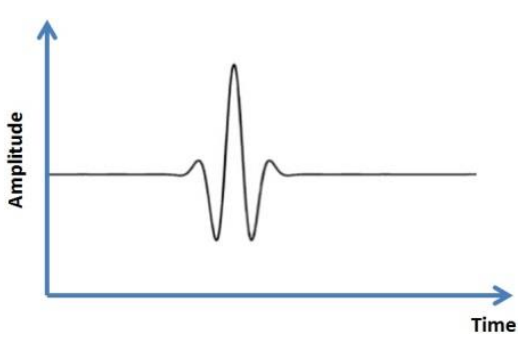

(a)

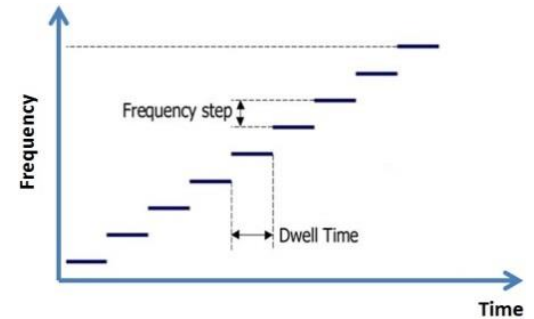

(b)

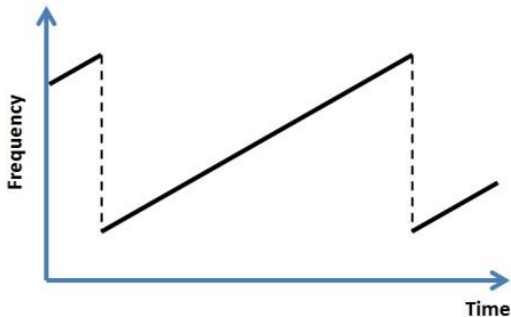

(c)

Fig. 2.3: (a) Impulse, (b) SFCW, and (c) FMCW waveforms.

In this research, we only focus on the first two types, i.e., impulse waveform and SFCW. Impulse waveform has advantage of fast measurement speed, but has limitation in the transmitted power. By contrast, SFCW has higher transmission power and more flexibility in spectrum shaping, but at the expense of slower measurement speed. In the existing GPR systems, both impulse and SFCW waveforms can be implemented in various frequency ranges depend on the corresponding applications. Since our focus is on infrastructure survey application, we use $1 \mathrm{GHz}$ as the center frequency for impulse waveform, and 3-6 GHz as the frequency range for SFCW waveform.

Impulse waveform can be used to directly obtain measurement data in time domain. There are several types of impulse waveform that are commonly utilized in GPR system. The most typical one is Gaussian waveform set, i.e., Gaussian and its derivatives. Besides Gaussian waveform set, some others impulse waveforms such as Hermite Polynomial [70] and Gegenbauer [80] have been used for UWB communication system.

Unlike impulse waveform that acquires the data in time domain, SFCW waveform provides measurement in frequency domain. In this technique, at a particular time instance a singlefrequency sinusoidal wave is emitted. The signal frequency increases step-by-step every fixed period of time. The frequency response of the target can be obtained by combining the received response over all frequencies.

In order to focus on the performance of each diversity independently, we only use one type of waveform for the investigation of spatial and polarization diversities. In the simulation, we use the first Gaussian derivatives waveform as shown in Fig. 2.4. This waveform can be formulated as in (2.2), where $f$ is the center frequency. Meanwhile, we use SFCW waveform for experimental data due to the hardware limitation.

$$
s(t)=-4 \pi^{2} f^{2} e^{1 /\left(8 \pi^{2} f^{2}\right)} e^{-2 \pi^{2} f^{2}(t-1 / f)^{2}}(t-1 / f)
$$




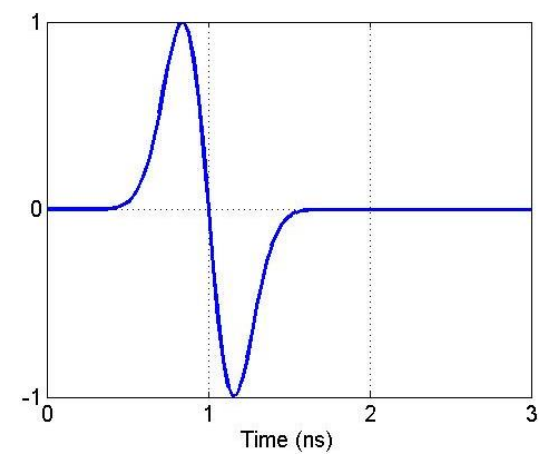

Fig. 2.4: First Gaussian derivative waveform.

On the other hand, in the study of waveform diversity we need to use several types of waveform. Therefore, we decide to use the first four order of Hermite Polynomial waveform set as shown in Fig. 2.5(a). The corresponding spectrums of these waveforms are given in Fig. 2.5(b).

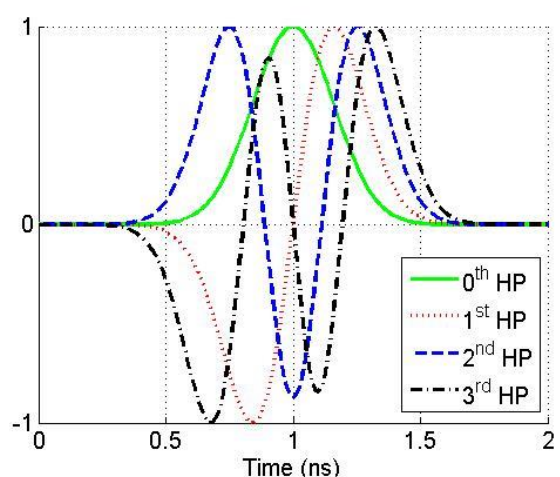

(a)

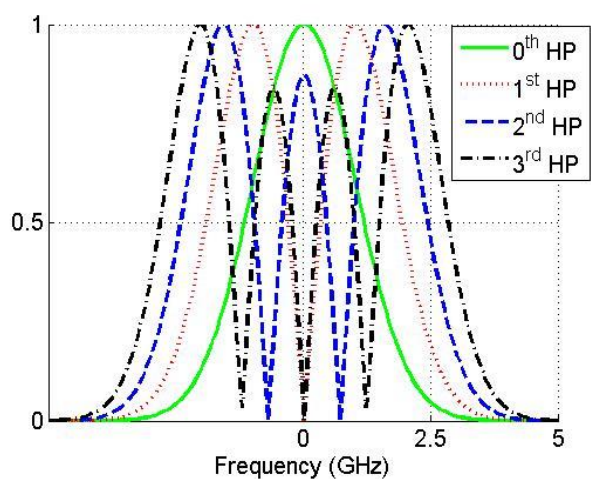

(b)

Fig. 2.5: Hermite polynomial waveforms in (a) time domain and (b) frequency domain.

\subsection{GPR Polarization}

Typically, the transmitter and receiver of GPR system only have single polarization, either linear or circular. Due to the difficulty in maintaining circular polarization over wide bandwidth, linear polarization is preferred for UWB GPR. However, some research has tried to develop fully-polarimetric GPR in which the measurement is conducted in combination of 2 polarization bases. This technique was inspired by polarimetric synthetic aperture radar system (PolSAR), where combinations of several polarization bases were used in remote sensing to obtain more information on the target. Some processing methods from PolSAR have been applied to fully-polarimetric GPR [71]-[75] and the results showed the improvement in image quality and target interpretation. 
In our study of spatial diversity and waveform diversity, we only use a single linear polarization to focus on the performance of those diversities independently. Meanwhile, in the study of polarization diversity, we will adapt data collection and signal processing techniques from PolSAR. Thus, fully-polarimetry measurement will be utilized in the investigation of polarization diversity.

\subsection{GPR Signal Processing}

GPR data can be represented in 3 different formats: A-scan, B-scan, and C-scan. A-scan is a single-trace data collected by a pair of transmitter and receiver at a certain point above the ground. The collection of several A-scans forms a 2D image which is known as B-scan. Cscan is the 3D data comprises of several B-scan. Fig. 2.6 illustrates the representation of GPR data in A-scan, B-scan, and C-scan.

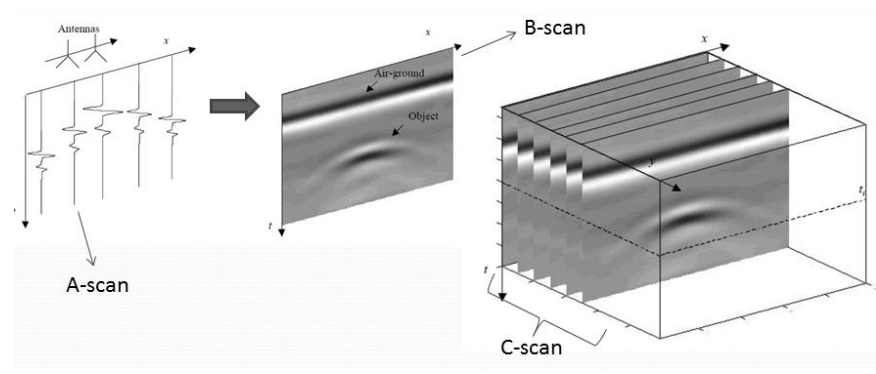

Fig. 2.6: A-scan, B-scan, and C-scan.

Basically, the main objective of GPR signal processing is to improve data interpretation and target detection. Although GPR data processing is a broad subject which depends on the applications of interest, generally signal processing of UWB GPR system can be classified into 3 main parts [81], [82] as shown in Fig. 2.7. The first part is preprocessing, in which the irregularities, clutter, and other unwanted components are removed. Preprocessing prepares the data for the next step, which can be migration or target detection. The second part is migration, which is aimed to constructs focused image from the target reflections. In some applications, preprocessing result is adequate to be used for target detection and classification, so the migration step can be skipped. However, migration step can be advantageous to enhance target reflection and improve data interpretation, especially for nontrained user, since it collects target reflections and return them to the correct position. Finally, the last step is target detection or classification. This step can involve target detection, feature extraction, target classification, and decision. 
In the following subsections, we present the necessary signal processing techniques for our study. The preprocessing part will be explained in detail. The migration method depends on array configuration, so we only give introduction of each migration algorithms in this chapter and provide the thorough detail in Chapter 3. Similarly, we utilize waveform and polarization diversities to develop the target classification methods in this study. Therefore, target classification part will not be covered in this chapter. The comprehensive detail of waveform diversity and polarization diversity will be given later in Chapter 4 and 5 respectively.

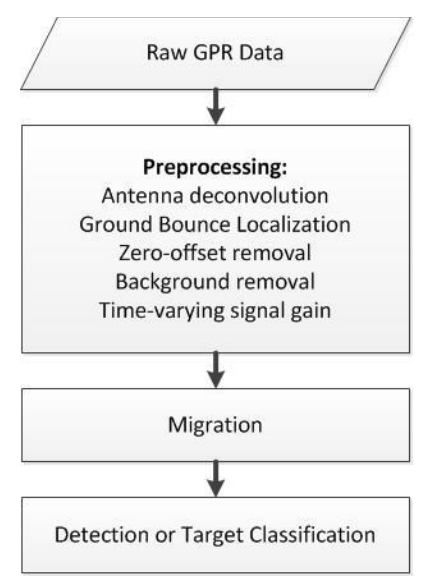

Fig. 2.7: Signal processing of GPR data.

\subsubsection{Preprocessing}

Due to unideal conditions in real measurement, a raw GPR data will be contaminated by clutter and noise in addition to the target reflection. Several factors influence the amount of clutter and irregularities in the data, such as antenna imbalance, thermal noise, ground bounce, ground reflection, ground attenuation, etc. The main objectives of preprocessing steps are to reduce these unwanted components and to enhance the signal-to-noise ratio (SNR).

Preprocessing incorporates several steps depend on the measurement purposes and condition.

Due to the ideal condition in the simulation and experimental data in this study, we only apply some common preprocessing methods. For simulation data, we only utilize zero-offset removal, background removal, and time-varying signal gain. Meanwhile, for experimental data, we use most of the preprocessing methods described in this subsection. The detail of each preprocessing technique is given as follows. 


\subsubsection{Antenna Deconvolution}

Practically, GPR antenna produces some distortion on the received signal. The frequencydependent response of the antenna will transform the shape of emitted waveform during the transmission and reception process. Moreover, the close distance between the antennas and the ground surface will generate coupling between the transmitter and receiver antennas. The antenna coupling component can be removed by first pointing the antenna into the air and measuring their independent coupling without target. Then, this coupling part can be subtracted from the A-scan data. Nevertheless, the antenna response still remains and additional processing method needs to be carried out.

We can model the whole transmission and reception process as a response of linear system with AWGN noise as expressed in (2.3). In (2.3), the antenna and transmission channel is modeled as a linear system with impulse response $H(\omega)$, while $Y(\omega)$ represents the received signal, $X(\omega)$ represents the original waveform, and $N(\omega)$ is the noise component.

$$
Y(\omega)=H(\omega) X(\omega)+N(\omega)
$$

One option to obtain the initial shape of the waveform is by using deconvolution. This method can be performed in time domain [83] or in frequency domain [84]. Yet, frequency domain deconvolution is faster due to the fast Fourier transform (FFT) algorithm.

The deconvolution in frequency domain is given by (2.4), where $Y(\omega)$ is the input signal in frequency domain, $H(\omega)$ is the antenna transfer function, $X_{\text {est }}(\omega)$ is the deconvolution output in frequency domain, and $\lambda$ is a parameter to control the balance between noise reduction and error reduction. The latter parameter can be a particular function or a constant. Some common approaches use $\lambda=$ constant and $\lambda=$ constant $\times \omega^{4}$, where the value of the constant can be determined by using frequency domain optimization technique [85].

$$
X_{e s t}(\omega)=Y(\omega) \frac{H^{*}(\omega)}{|H(\omega)|^{2}+\lambda}
$$

To demonstrate the antenna deconvolution technique, we generate the A-scan data from finite-difference time-domain (FDTD) simulation by using GPRMax [86] with bow-tie antenna as shown in Fig. 2.8. The antenna transmits a Ricker impulse waveform with center frequency of $1 \mathrm{GHz}$. Some noise is added to the received data and the deconvolution method 
is carried out afterwards. The result is given in Fig. 2.9 with $\lambda=$ constant. We can observe that the deconvolution result is approximately equal to Ricker impulse, which is the original waveform that passed through the transmitter antenna.

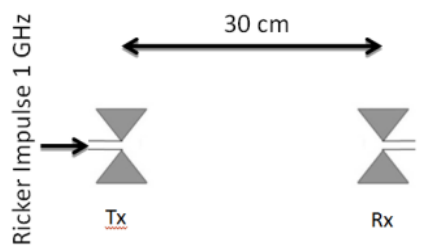

Fig. 2.8: Simulation configuration of antenna deconvolution.

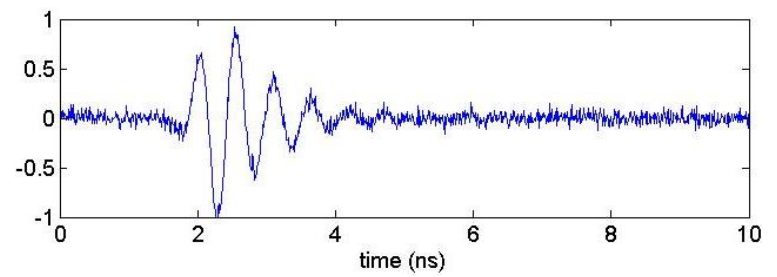

(a)

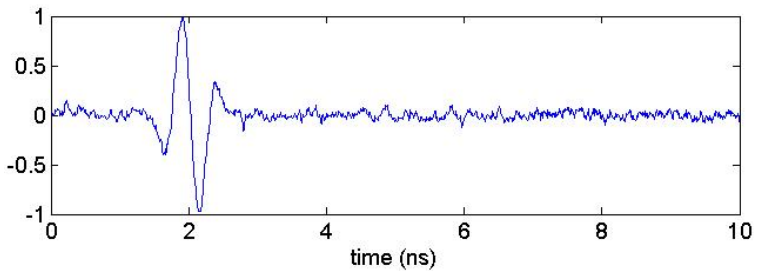

(b)

Fig. 2.9: Simulation result of (a) received signal and (b) deconvolution.

\subsubsection{Ground Bounce Localization}

During data collection process, the roughness of ground surface can cause displacement on the distance of the sensors to the ground. Such displacement produces variation of zero position on each A-scan. Therefore, we need to adjust each A-scan to eliminate this variation [87], [88].

Ground reflection is commonly used to determine the zero position. However, practically there are several points from ground reflection that can be used as reference, such as first break position, first positive peak, first negative peak, zero amplitude point, and midamplitude point. In our case, we use the first maximum value in each A-scan as the estimator of ground position as given in (2.5), where $x_{i}(n)$ is the original $i$-th A-scan and $n_{\text {peak }}$ is the time position of the peak value.

$$
n_{\text {peak }}=\max _{n} x_{i}(n)
$$

Then, the A-scan is shifted according to this estimator of zero position,

$$
s_{i}(n)=x_{i}\left(n+n_{\text {peak }}\right)
$$


where $s_{i}(n)$ is the $i$-th A-scan after adjustment. To show the implementation of groundbounce localization technique, a simulation data is generated by using GPRMax with target configuration given in Fig. 2.10. Then, noise and ground bounce are added to the data, followed by ground bounce localization.

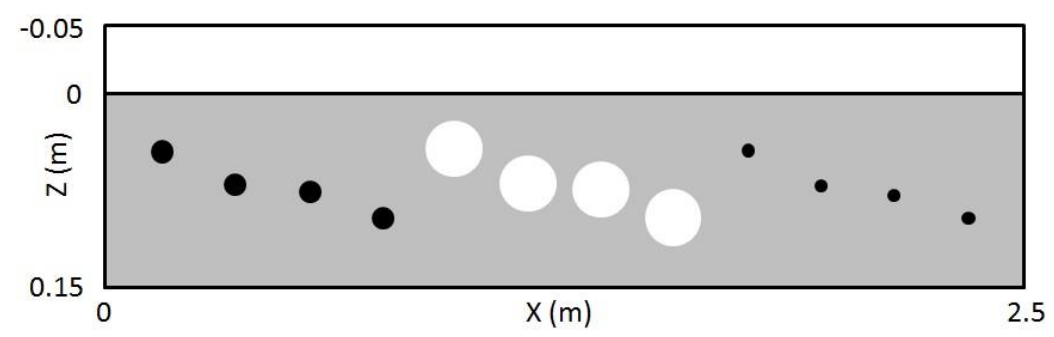

Fig. 2.10: Target configuration in the simulation for data preprocessing.

The result of ground bounce localization is given in Fig. 2.11. As we can see, the irregularities in the A-scan position are successfully eliminated.

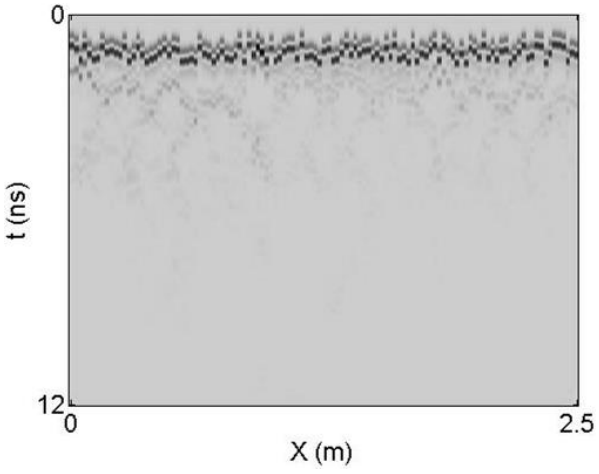

(a)

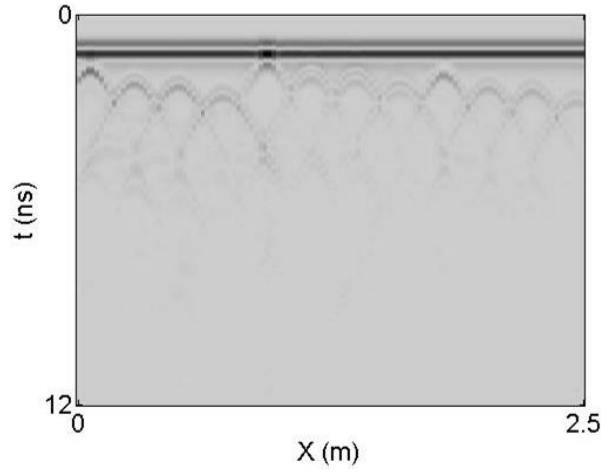

(b)

Fig. 2.11: (a) Simulation result and (b) ground-bounce localization result.

\subsubsection{Zero-Offset Removal}

Some of the signal processing algorithms, such as f-k migration and time-varying signal gain, are not invariant to DC component in the signal. Therefore, DC component should be removed before proceeding to those processing steps. A common zero-offset removal method is by using mean estimator of each A-scan. Then, this mean estimator is subtracted from the corresponding A-scan as formulated in (2.7), where $\hat{s}_{i}{ }^{\prime}$ is the output vector of $i$-th A-scan, $\hat{s}_{i}$ is the input vector of $i$-th A-scan, and $s_{i}(n)$ is the $n$-th time sample of the $i$-th A-scan.

$$
\hat{S}_{i}^{\prime}=\hat{s}_{i}-\left(\frac{1}{N} \sum_{n=1}^{N} s_{i}(n)\right)
$$




\subsubsection{Background Removal}

The raw GPR data also contains reflection from ground surface, ground layer, and clutter. These components are considered as background and should be removed in order to enhance the feature from the target of interest. Several techniques are available to estimate background components, such as mean-based estimator, median-based estimator, and Principal Component Analysis (PCA) [84], [89], [90]. The simplest method is mean-based estimator as formulated in (2.8), where $\hat{x}_{b a c k}$ is the estimator of the background, $x_{i}$ is the $i$-th A-scan signal, and $N$ is the number of A-scan.

$$
\hat{x}_{b a c k}=\frac{1}{N} \sum_{i} x_{i}
$$

Then, the background component can be removed by subtracting $\hat{x}_{b a c k}$ from each A-scan as given in (2.9).

$$
x_{i}{ }^{\prime}=x_{i}-\hat{x}_{b a c k}
$$

Compared to mean-based estimator, PCA provides more reliable background estimation for non-homogeneous medium case. This method estimates the background component based on eigenvalue decomposition on covariance matrix of the A-scan. Let the column vector $x_{i}$ denotes the $i$-th A-scan signal, and $X$ denotes the B-scan matrix whose columns are $x_{i}$. Then, we can estimate the covariance matrix $(C)$ of the B-scan data as

$$
C=\sum_{i}\left(x_{i}-m_{x}\right)\left(x_{i}-m_{x}\right)^{H}
$$

where $m_{x}$ is the mean estimator of $x_{i}$ and is given by

$$
m_{x}=\frac{1}{N} \sum_{i} x_{i}
$$

The covariance matrix can be decomposed as

$$
C=Q V Q^{H}=\sum_{i} \lambda_{i} q_{i} q_{i}^{H}
$$

where $V$ is the diagonal matrix that contains the eigenvalues of $C, Q$ is a matrix whose columns are eigenvectors of $C, q_{i}$ is the eigen-vector of $C$, and $\lambda_{i}$ is the eigen-value of $C$. Based on this decomposition, we can separate the covariance matrix into 2 components as 
given in (2.13) with assumption $\lambda_{i} \geq \lambda_{i+1}$. The first component on the right hand side is assumed to be the target component while the latter one is considered as the background component.

$$
C=\sum_{i=1}^{p} \lambda_{i} q_{i} q_{i}{ }^{H}+\sum_{i=p+1}^{N} \lambda_{i} q_{i} q_{i}{ }^{H}
$$

We select the value of $p$ so the target component contains $95 \%$ of the signal energy as given in (2.14).

$$
\frac{\sum_{i=1}^{p} \lambda_{i}}{\sum_{i=1}^{N} \lambda_{i}} \geq 95 \%
$$

To obtain the target component from the A-scan signal $x_{i}$, we can define a projection operator

$$
P=\sum_{i=1}^{p} q_{i} q_{i}^{H}
$$

Then, the target component in the A-scan is given by (2.16).

$$
X^{\prime}=P\left(X-m_{x}\right)
$$

Fig. 2.12 shows the result of mean-based estimator and PCA that are applied to the simulation data in Fig. 2.11b. As one can see, PCA gives better noise and clutter suppression compared to mean-based estimator. However, PCA can also potentially eliminate the target component.

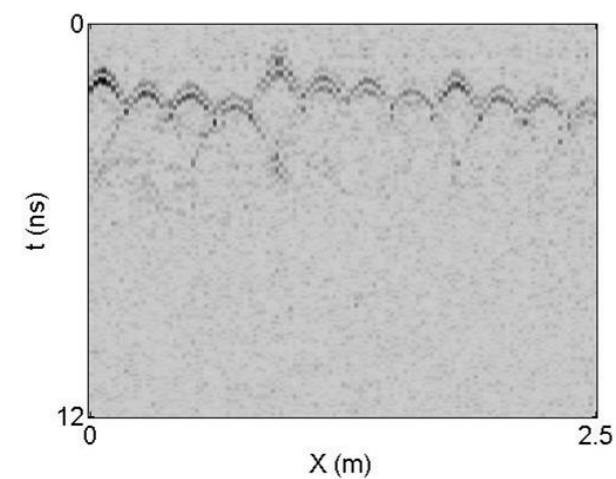

(a)

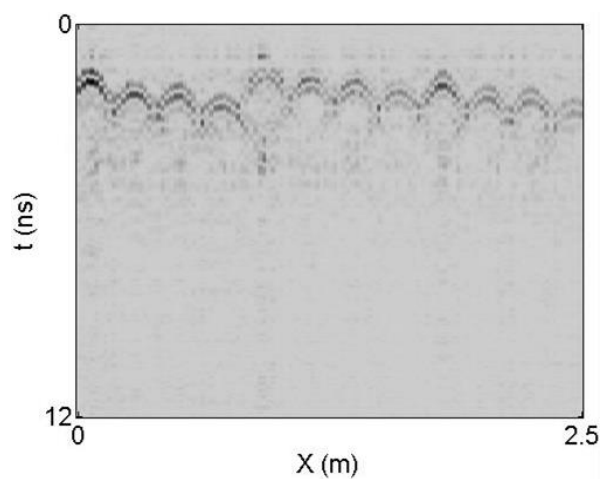

(b)

Fig. 2.12: Result of background removal by using (a) mean-based estimator and (b) PCA. 


\subsubsection{Time-Varying Signal Gain}

The electromagnetic wave that propagates through subsurface will be attenuated due to the medium loss factor and spreading of the wave energy over its wave-front. Thus, to enhance the reflection of the deeper target, the A-scan signal should be adjusted by using timedependent signal gain. This gain can be implemented based on record data that has a known gain function.

\subsubsection{Migration}

A point target in the GPR data will appear as a hyperbolic due to the antenna beam-width and the movement of the sensor during data acquisition process. The objective of migration step is to convert this hyperbolic diffraction into focused image. As described before, in several applications where the main objective is to detect the object, the migration part can be omitted. However, if the target size, location, and shape are crucial information, then migration becomes an important part in data processing chain. Furthermore, for some case such as UXO detection, migration can be advantageous to enhance the target reflection and improve target detection performance.

Migration was originally developed in seismic imaging field and has been used widely for GPR signal processing. Several of the common migration methods are Kirchhoff migration, f-k migration, and phase-shift migration. Since these migration algorithms depend on sensor configuration, we only present an introduction of each algorithm herein and postpone the brief formulations until Chapter 3.

\subsubsection{Kirchhoff Migration}

Kirchhoff migration was formulated based on integral solution of wave equation by using Green's Function [91]. This algorithm provides the best flexibility in handling various sensor configurations, domain size, and inhomogeneous medium. Yet, Kirchhoff migration has drawback in terms of computational complexity compared to $\mathrm{f}-\mathrm{k}$ migration and phase-shift migration.

\subsubsection{F-k Migration}

In $\mathrm{f}-\mathrm{k}$ migration, the focusing process is mainly carried out in frequency and wavenumber domain [92]. Due to the utilization of FFT algorithm, f-k migration is the fastest method 
among all migration algorithms described in this study. However, since this algorithm is based on homogeneous medium assumption, the migration result will degrade under inhomogeneous medium case.

\subsubsection{Phase-Shift Migration}

Phase-shift migration is based on downward continuation principle. The focusing is performed in iterative manner over vertical dimension by a particular phase-shift operator. Therefore, this method can handle vertically varying medium case [93]. In terms of computational speed, this migration algorithm lies between f-k migration and Kirchhoff migration.

\subsection{GPR Performance Measurement}

The assessment of GPR performance with particular configurations must be performed based on qualitative and quantitative measures. Thus, we select several relevant parameters from remote sensing and seismic imaging field to be used in analyzing the GPR images. Based on the scope of the study, the selected performance parameters are resolution, integrated sidelobe ratio (ISLR), peak side-lobe ratio (PSLR), dip-angle range, and target characterization ability. The first three parameters can be calculated quantitatively from the point spread function (PSF) of the image, which is the imaging result on a point target. Dip-angle range can be observed from the imaging result on rotated planar target, while its rough approximation formula is also available. Finally, the last parameter can only be observed qualitatively.

\subsubsection{Resolution}

Resolution indicates the minimum distance between 2 targets that can still be resolved by the radar system. Since there are various definitions of resolution, in this study we use 3-dB resolution as the quantitative measure. The slice of the PSF in Fig. 2.13 [94] illustrates the calculation of $3-\mathrm{dB}$ resolution as the width of the main lobe that exceeds $3-\mathrm{dB}$. 


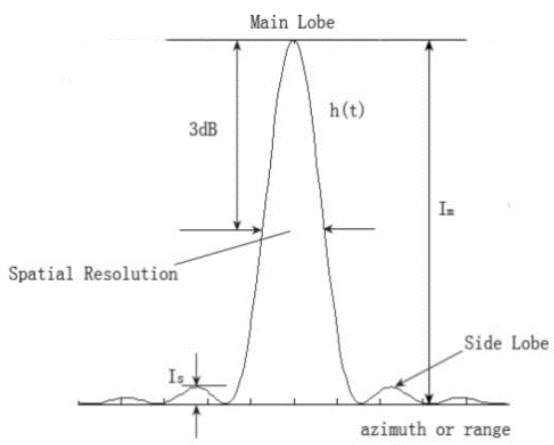

Fig. 2.13: Slice of PSF.

\subsubsection{ISLR and PSLR}

In SAR remote sensing, ISLR is defined as the ratio between the energy in the side-lobes and main-lobe, while PSLR is the ratio between the peak of the main-lobe and the peak of the first side-lobe. Both parameters can be extracted from PSF. By referring to Fig. 2.13, the formulations of ISLR and PSLR are given in (2.17) and (2.18) respectively.

$$
\begin{gathered}
I S L R=10 \log _{10}\left(\frac{\int_{-\infty}^{a}|h(t)|^{2} d t+\int_{b}^{\infty}|h(t)|^{2} d t}{\int_{a}^{b}|h(t)|^{2} d t}\right) \\
P S L R=20 \log _{10}\left(\frac{I_{s}}{I_{m}}\right)
\end{gathered}
$$

\subsubsection{Dip-Angle Range}

Although a radar system can image a point target, it might not be able to resolve a surface with a particular inclination angle. The dip-angle range indicates the maximum inclination angle of a surface that can still be resolved by the radar. This parameter is adopted from seismic imaging and depends on sensor configuration and array length.

\subsubsection{Target Characterization}

The last aspect that we consider in this study is the target characterization ability. Specifically, we will explore the capability of a certain processing method in distinguishing various target shapes and materials. 


\subsection{Concluding Remarks}

This chapter has presented the fundamental theory of spatial, waveform, and polarization diversities. Based on the described background, we define the implementation approach on each diversity in this study. The spatial diversity utilizes the concept on MIMO array. In the waveform diversity, we process the measurement data collected by 4 different impulse waveforms. For polarization diversity, we adopt the polarimetric decomposition methods developed in PolSAR.

We have also discussed the basic principle of signal processing methods in this study. We provide the preprocessing part in detail, while the migration part and target detection or classification part are postponed until Chapter 3, 4, and 5. Finally, we have formulated several performance parameters that will be used to assess the performance of each diversity technique. 


\section{CHAPTER 3}

\section{The Spatial Diversity for UWB GPR Systems}

As discussed in Chapter 2, the MIMO technology is an advanced concept to implement spatial diversity on GPR system. In this chapter, we introduce MIMO configurations for UWB GPR and present a comprehensive investigation on the MIMO imaging performance and several related signal processing aspects. It begins with a review of existing theories regarding GPR array configuration, migration methods, and velocity estimation method in Section 3.1. Then, the study of the MIMO capability for UWB GPR is given in Section 3.2 which covers the performance analysis on several imaging parameters. The influence of migration algorithms on the imaging results is investigated in Section 3.3. In Section 3.4, an autofocusing algorithm is proposed to resolve velocity estimation issue in MIMO configurations. Finally, Section 3.5 summarizes all the results in this chapter.

\subsection{Review of Existing Theory}

\subsubsection{Sensor Configurations on GPR System}

Traditionally, GPR system utilizes the so-called SISO configuration, in which a transmitter only works with one receiver. Such configuration can be realized by a single pair of transmitter and receiver or by using an array of sensors. Another simplified version of GPR array configuration is SIMO. In SIMO configuration, the array consists of only one transmitter and several receivers that work simultaneously. Thus, the data collection can be carried out in single transmission and reception process.

MIMO is an emerging technology in communication and radar systems where several transmitters and receivers are allowed to work together. By such flexibility, there are many ways to implement MIMO arrays. In this study, we consider a MIMO array that comprises several pairs of transmitter and receiver. In contrast to SISO configuration, MIMO configuration enables all transmitters and receivers to correlate. The transmitters will emit signal sequentially so that there is only one active transmitter at a particular time instance. Meanwhile, all the receivers collect the data simultaneously. The transmitting and receiving schemes of SISO, SIMO, and MIMO arrays have been illustrated by Fig. 2.2. 


\subsubsection{Migration Formula of SISO, SIMO, and MIMO Configurations}

Migration is an essential part in GPR signal processing by which hyperbolic diffractions in raw GPR data are converted into focused image. Originated from seismic imaging field, currently there are various versions of migration algorithm that have been developed. In this research, we consider only 3 migration methods that are common in GPR signal processing: Kirchhoff migration, phase-shift migration, and f-k migration. Furthermore, we take the formulations of these migration algorithms from Seismic imaging field as given in [95].

The formulations of migration algorithms depend on sensor configuration. In seismic imaging, sensor configuration is defined as gather. SISO configuration is equivalent to zero-offset gather in seismic imaging, while SIMO configuration is equivalent to common-source gather [96]. Although there is no equivalent gather for MIMO configuration, we can apply the concept of pre-stack migration and zero-azimuth pre-stack migration to handle such cases.

We use the following notations for all the migration algorithms in this thesis. The array lies over $x$-axis and moves in direction of $y$-axis as shown in Fig. 3.1. The depth direction is given by $z$-axis. The received data is denoted by $u(x, z, t)$ for $2 \mathrm{D}$ case and $u(x, y, z, t)$ for $3 \mathrm{D}$ case. The migration output is denoted by $u_{m}(x, z, t)$ for $2 \mathrm{D}$ case and $u_{m}(x, y, z, t)$ for $3 \mathrm{D}$ case. The wave velocity is denoted by $c . k_{x}$ denotes the wavenumber of $x, k_{y}$ denotes the wavenumber of $y$, and $k_{z}$ denotes the wavenumber of $z . \omega$ is the frequency variable, which is the Fourier transform pair of time variable $t$. For SISO array, the transmitter and receiver are collocated, so their location in $x$-axis is denoted by $x_{0}$. For SIMO and MIMO arrays, the transmitter and receiver can have different position, so we need to assign separated notation for their positions in $x$-axis. The position of transmitter is denoted by $x_{s}$ with $k_{x s}$ as its wavenumber counterpart, while the position of receiver is denoted by $x_{g}$ with $k_{x g}$ as its corresponding wavenumber. Alternatively, these positions can be represented in terms of mid-point $x$ and offset $x_{h}$ with Fourier transform pair $k_{x}$ and $k_{x h}$ respectively. The relation between $\left(x_{s}, x_{g}\right)$ and $\left(x, x_{h}\right)$ is given by (3.1), while the relation between $\left(k_{x s}, k_{x g}\right)$ and $\left(k_{x}, k_{x h}\right)$ is given by (3.2).

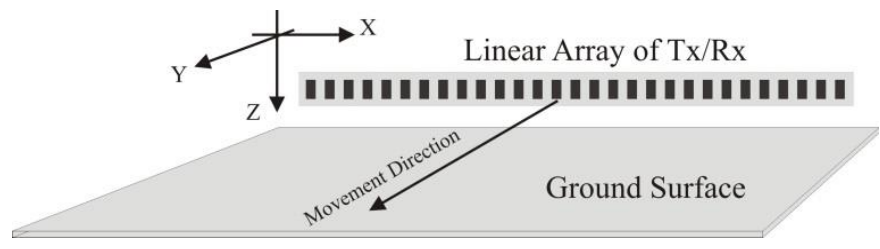

Fig. 3.1: GPR array configuration and movement direction. 


$$
\begin{gathered}
x=\frac{x_{g}+x_{s}}{2}, x_{h}=\frac{x_{g}-x_{s}}{2} \\
k_{x}=k_{x g}-k_{x s}, k_{x h}=k_{x g}+k_{x s}
\end{gathered}
$$

\subsubsection{Kirchhoff Migration}

Kirchhoff migration is a migration algorithm based on solution of wave equation by using Green's function. This migration method provides high flexibility in terms of handling arbitrary configuration and medium inhomogeneity. However, this algorithm is computationally expensive compared to phase-shift migration and f-k migration.

Conceptually, the principle of this algorithm can be illustrated by Fig. 3.2. First, the data is filtered. Then, focusing process is carried out on each point in the output data. This process involves calculation of travel time of the wave from the transmitter to the corresponding output point, and return to the receiver. Finally, we accumulate all the received data at the corresponding travel time.

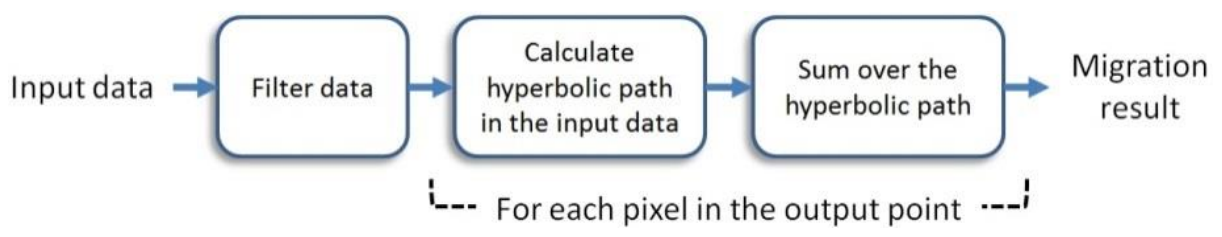

Fig. 3.2: Process of Kirchhoff migration.

For SISO case, Kirchhoff migration for zero-offset configuration is given by (3.3) for 2D case and (3.4) for 3D case. In (3.3) and (3.4), $r$ is the distance from input point to output point given by (3.5), while $d_{h}$ is a half-derivative filter given by (3.6).

$$
\begin{gathered}
u_{m}(x, z)=\int \frac{4 z}{\sqrt{2 \pi c r^{3}}}\left[d_{h-}^{*} u\right]\left(x_{0}, z_{0}=0, t_{0}=\frac{r}{c}\right) d x_{0} \\
u_{m}(x, y, z)=\int \frac{4 z}{\sqrt{2 \pi c r^{3}}}\left[d_{h-} * u\right]\left(x_{0}, y_{0}, z_{0}=0, t_{0}=\frac{r}{c}\right) d x_{0} d y_{0} \\
r=\sqrt{\left(x-x_{0}\right)^{2}+\left(y-y_{0}\right)^{2}+z^{2}} \\
{\left[d_{h \pm} * u\right](t)=\int \sqrt{\mp i \omega} u(\omega) e^{-i \omega t} d \omega}
\end{gathered}
$$

For SIMO case, Kirchhoff migration for common-source gather is given by (3.7) for 2D case and (3.8) for 3D case, where $r$ is given by (3.9). 


$$
\begin{gathered}
u_{m}(x, z)=\int \frac{2 z \sqrt{r_{s}\left(r_{s}+r_{g}\right)}}{\sqrt{2 \pi c r_{g}^{3}}}\left[d_{h-} * u\right]\left(x_{0}, z_{0}=0, t_{0}=\frac{r_{s}+r_{g}}{c}\right) d x_{0} \\
u_{m}(x, y, z)=\int \frac{z\left(r_{s}+r_{g}\right)}{8 \pi c}\left[\frac{\left(x_{g}-x_{s}\right)\left(x-x_{g}\right)}{r_{s} r^{3}}+\frac{1}{r_{g}}\left(\frac{1}{r_{s}}+\frac{1}{r_{g}}\right)\right] u\left(x_{0}, y_{0}, z_{0}=0, t_{0}=\frac{r_{s}+r_{g}}{c}\right) d x_{0} d y_{0} \\
r=r_{s}+r_{g}=\sqrt{\left(x-x_{s}\right)^{2}+\left(y-y_{s}\right)^{2}+z^{2}}+\sqrt{\left(x-x_{g}\right)^{2}+\left(y-y_{g}\right)^{2}+z^{2}}
\end{gathered}
$$

For MIMO case, Kirchhoff pre-stack migration is given by (3.10) for 2D case and (3.11) for $3 \mathrm{D}$ case, where $r_{s}$ is the distance from transmitter to output point given by (3.12), $r_{g}$ is the distance from receiver to output point given by (3.13), and $d_{h+}$ is a half-derivative filter given by (3.6).

$$
\begin{gathered}
u_{m}(x, z)=\frac{z^{2}}{2 \pi c} \iint \frac{\left[d_{h+} * u\right]\left(x_{s}, x_{g}, t=\frac{r_{s}+r_{g}}{c}\right)}{\left(r_{s} r_{g}\right)^{3 / 2}} d x_{s} d x_{g} \\
u_{m}(x, y, z)=\frac{z^{2}}{2 \pi c} \iint \frac{\left[d_{h+}^{*} u\right]\left(x_{s}, x_{g}, y, t=\frac{r_{s}+r_{g}}{c}\right)}{\left(r_{s} r_{g}\right)^{3 / 2}} d x_{s} d x_{g} d y \\
r_{s}=\sqrt{\left(x-x_{s}\right)^{2}+\left(y-y_{s}\right)^{2}+z^{2}} \\
r_{g}=\sqrt{\left(x-x_{g}\right)^{2}+\left(y-y_{g}\right)^{2}+z^{2}}
\end{gathered}
$$

\subsubsection{Phase-Shift Migration}

In phase-shift migration, the focusing is formulated based on exploding reflector model (ERM) and downward-continuation principle [93]. The concept of ERM considers the measurement data as wave-field that expands from the target and collected at $z=0$. Thus, to obtain the target location, this wave-field must be interpolated downward by multiplication with a particular phase-shift operator.

Due to the utilization of FFT algorithm, phase-shift migration has advantages of faster computational speed compared to Kirchhoff migration. Unfortunately, this method has several drawbacks, i.e., it can handle only vertically varying wave-velocity and is applicable only on limited sensor configurations. Although this migration technique is not available for SIMO configuration, in Section 3.2 we develop a migration algorithm for such configuration which has equivalent formulation to phase-shift migration. 
For SISO configuration, the steps of zero-offset phase-shift migration are presented in Fig. 3.3. The measurement data is first transformed into frequency and wavenumber domain by using FFT algorithm. Then, the interpolation is carried out in iterative manner for every depth point in the output data. This interpolation process is implemented by multiplying the previous data with phase-shift operator given in (3.14), where $k_{z}$ is given by (3.15) for 2D case and (3.16) for 3D case. Finally, the data is transformed back into space domain by applying IFFT on $x$ and $y$ dimensions, followed by summation over $\omega$.

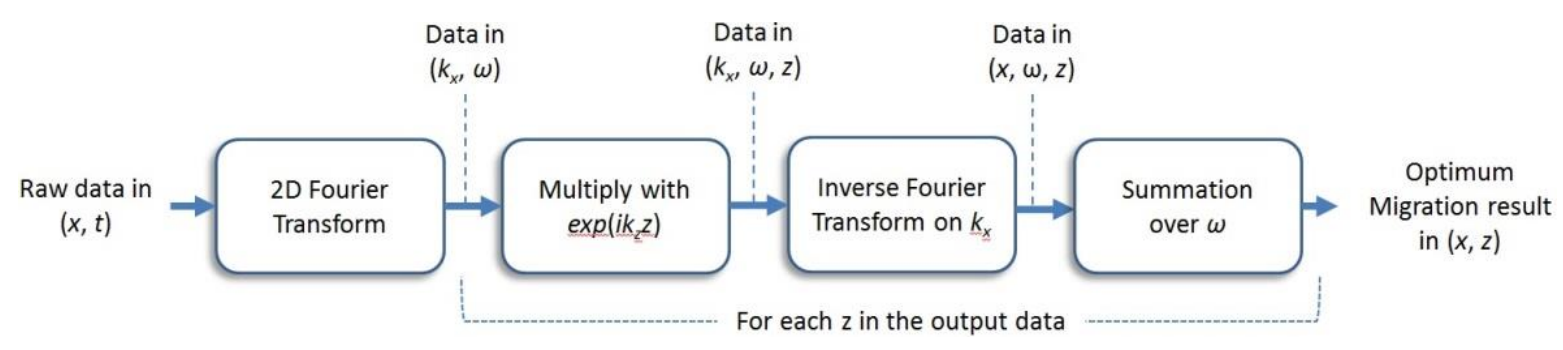

Fig. 3.3: Phase-shift migration for SISO configuration.

$$
\begin{gathered}
u\left(k_{x}, k_{y}, z, \omega\right)=u\left(k_{x}, k_{y}, z=0, \omega\right) e^{-i k_{z} z} \\
k_{z}=-\frac{2 \omega}{c} \sqrt{1-\left(\frac{c k_{x}}{2 \omega}\right)^{2}} \\
k_{z}=-\frac{2 \omega}{c} \sqrt{1-\left(\frac{c k_{x}}{2 \omega}\right)^{2}-\left(\frac{c k_{y}}{2 \omega}\right)^{2}}
\end{gathered}
$$

For MIMO configuration, the pre-stack phase-shift migration is shown in Fig. 3.4. Similar to SISO case, the data is first transformed into frequency and wavenumber domain by using 3D or 4D FFT. Then, for every $z$ in the output data, the interpolation is applied by multiplication with phase-shift operator as given in (3.17), where $k_{z}$ is given by (3.18) for 2D case and (3.19) for 3D case. In MIMO configuration, the input data is one dimensional higher than the output data. This dimension is reduced by first changing the axis from $\left(k_{x s}, k_{x g}\right)$ to $\left(k_{x}=k_{x s}-\right.$ $k_{x g}, k_{x h}=k_{x s}+k_{x g}$ ), then perform summation over $k_{x h}=k_{x s}+k_{x g}$. Finally, to obtain the spatial domain result, IFFT is applied on $k_{x}$ and $k_{y}$ followed by summation over $\omega$. 


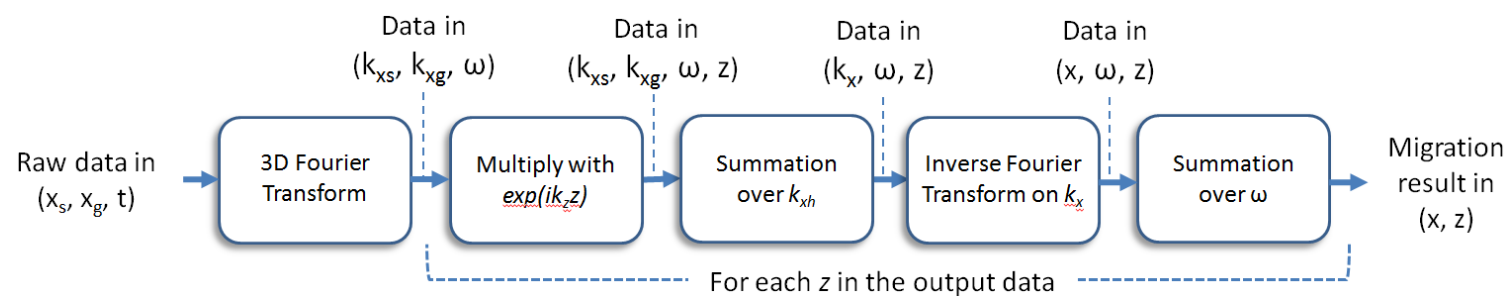

Fig. 3.4: Phase-shift migration for MIMO configuration.

$$
\begin{gathered}
u\left(k_{x s}, k_{x g}, k_{y}, z, \omega\right)=u\left(k_{x s}, k_{x g}, k_{y}, z=0, \omega\right) e^{-i k_{z} z} \\
k_{z(2 D)}=k_{z g}-k_{z s}=-\frac{\omega}{c}\left(\sqrt{1-\left(\frac{c k_{x g}}{\omega}\right)^{2}}+\sqrt{1-\left(\frac{c k_{x s}}{\omega}\right)^{2}}\right) \\
k_{z(3 D)}=k_{z(2 D)} \sqrt{1-\left(\frac{k_{y}}{k_{z(2 D)}}\right)^{2}}
\end{gathered}
$$

\subsubsection{F-k Migration}

F-k migration is a simplification of phase-shift migration for constant wave-velocity case. This migration method has the best efficiency in computational complexity compared with Kirchhoff migration and phase-shift migration. However, $\mathrm{f}-\mathrm{k}$ migration yields optimum result only on homogeneous medium case and is available only on limited sensor configurations.

F-k migration is derived from phase-shift migration. By not losing the general concept, the full formulation of phase-shift migration can be expressed as in (3.20), where $\tilde{\mathbf{k}}$ is the vector of horizontal wavenumber (not including $\left.k_{z}\right), \boldsymbol{\rho}$ is the vector of horizontal position, $\boldsymbol{r}=(x, y, z)$ for $3 \mathrm{D}$, and $\boldsymbol{r}=(x, z)$ for $2 \mathrm{D}$. F-k migration can be obtained from (3.20) by changing the integration variable from $\omega$ to $k_{z}$ as given in (3.21).

$$
\begin{gathered}
u_{m}(\mathbf{r})=\int u(\tilde{\mathbf{k}}, \omega) e^{-i \tilde{\mathbf{k}} \bullet \boldsymbol{\rho}-i k_{z} z} d \tilde{\mathbf{k}} d \omega \\
d \omega=\left|\frac{\partial \omega}{\partial k_{z}}\right| d k_{z}
\end{gathered}
$$

For SISO case, zero-offset f-k migration steps are shown in Fig. 3.5. Similar to phase-shift migration, the data is first transformed into frequency and wavenumber domain. Then, the socalled Stolt's interpolation is performed in which the data over $\omega$ is transformed into data over $k_{z}$. Finally, IFFT is applied to transform the data into spatial domain. The entire steps 
can be formulated by (3.22) for $2 \mathrm{D}$ case and (3.23) for 3D case, where $k_{z}$ is given by (3.15) and (3.16) for 2D case and 3D case respectively.

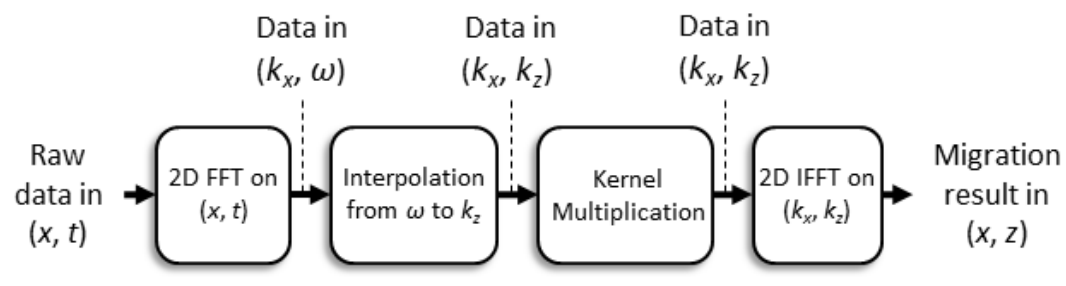

Fig. 3.5: F-k migration for SISO configuration.

$$
\begin{gathered}
u_{m}(x, z)=\iint\left|\frac{c^{2} k_{z}}{4 \omega}\right| u\left(k_{x}, \omega\right) e^{-i k_{x} x-i k_{z} z} d k_{x} d k_{z} \\
u_{m}(x, y, z)=\iiint\left|\frac{c^{2} k_{z}}{4 \omega}\right| u\left(k_{x}, k_{y}, \omega\right) e^{-i k_{x} x-i k_{y} y-i k_{z} z} d k_{x} d k_{y} d k_{z}
\end{gathered}
$$

For MIMO case, the migration steps are given in Fig. 3.6. The algorithm begins with transformation of the data into frequency-wavenumber domain. Then, Stolt's interpolation is applied to transform the data from $\omega$ to $k_{z}$, followed by summation over $k_{x h}=k_{x s}+k_{x g}$. Finally, IFFT is performed to transform the data into spatial domain. For 2D case, the formulation of $\mathrm{f}-\mathrm{k}$ migration can be expressed as in (3.24) with $k_{z}$ given in (3.18). Meanwhile, for 3D case the zero-azimuth f-k migration can be formulated as in (3.25) with $k_{z}$ given in (3.19).

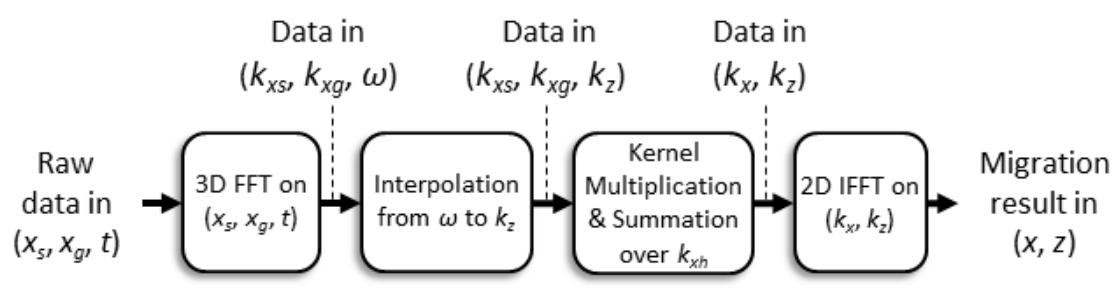

Fig. 3.6: F-k migration for MIMO configuration.

$$
\begin{gathered}
u_{m}\left(k_{x}, k_{z}\right)=\frac{1}{4 \pi} \int\left|\frac{c^{2} k_{z s} k_{z g}}{k_{z}}\right| u\left(k_{x}, k_{x h}, \omega\right) d k_{x h} \\
\sqrt{z} u_{m}\left(k_{x}, k_{y}, k_{z}\right)=\frac{c^{2}}{4 \sqrt{2}|\omega| \pi^{3 / 2}} \int\left|\frac{k_{z s} k_{z g}}{k_{z}}\right|^{3 / 2}\left(1-\frac{k_{y}{ }^{2}}{k_{z 2 D}{ }^{2}}\right)^{5 / 4} e^{i \frac{\pi}{4} \operatorname{sgn} \omega} u\left(k_{x}, k_{x h}, k_{y}, \omega\right) d k_{x h}
\end{gathered}
$$




\subsubsection{Velocity Estimation Method}

In migration process, wave velocity plays an important role in determining the migration result. Incorrect wave velocity can degrade the final image after migration process. Unfortunately, in most of real situations, wave velocity in the subsurface is unknown, and therefore a method to estimate wave velocity value becomes necessary in GPR signal processing.

The most common velocity estimation approach is by collecting the data in common midpoint (CMP) gather as illustrated in Fig. 3.7 [18]. This method assumes that the medium is horizontally layered. Due to the change in wave velocity, these layers will reflect the incident wave. The received data from each pair of sensor will appear as a hyperbolic as shown in Fig. 3.7 .

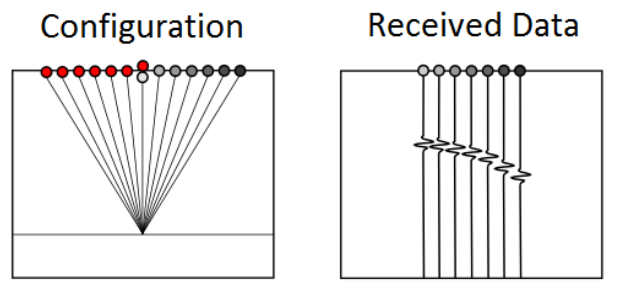

Fig. 3.7: CMP measurement and result.

In CMP gathers, each pair of sensor has different offset $(X)$ between the transmitter and the receiver, and thus the travel time of the reflected wave from the $i$-th sensor $\left(T_{i}\right)$ can be formulated by (3.26). In (3.26), $T_{0}$ is so-called as normal incident time, which is the travel time from the zero-offset sensor, while $V_{r m s}$ is the root-mean-square (rms) velocity of the medium.

$$
T_{i}^{2}=T_{0}^{2}+\frac{X^{2}}{V_{r m s}{ }^{2}}
$$

Based on (3.26), we can infer that the curvatures of the hyperbolic in the data are related to the wave velocity of the medium. In [97], such hyperbolic information is transformed from time and offset distance domain into normal incidence time and rms velocity domain by using (3.26) and a particular semblance filter. This process yields a velocity spectrum, and the optimum rms velocity can be obtained from the maximum value of the spectrum. Finally, the rms velocity can be related to the velocity of each layer by 


$$
V_{r m s}\left(\tau_{N}\right)^{2}=\frac{\sum_{i=1}^{N} v\left(\tau_{i}\right)^{2} \Delta \tau_{i}}{\sum_{i=1}^{N} \Delta \tau_{i}}
$$

where $V_{r m s}\left(\tau_{N}\right)$ is the rms velocity at $N$-th layer, $v\left(\tau_{i}\right)$ is the wave velocity at $i$-th layer, $\Delta \tau_{i}$ is the time thickness of the $i$-th layer. Then, we can estimate the velocity at layer- $i$ by using Dix formula [98] as given in (3.28).

$$
v\left(\tau_{i}\right)^{2}=\frac{\tau_{i} V_{r m s}\left(\tau_{i}\right)^{2}-\tau_{i-1} V_{r m s}\left(\tau_{i-1}\right)^{2}}{\Delta \tau_{i}}
$$

Another common method is by estimating the velocity directly from the reflection of the target. Specifically, optimization is carried out on particular focusing parameters as an indicator of correct wave velocity [99]-[102]. In [101], image entropy is used as focusing parameter for landmine detection, while in [102] several focusing parameters are compared for detection of rebars inside bridge. This method offers some advantages compared to semblance approach, as there is no additional measurement data required in estimating wave velocity and the image quality can be optimized directly.

\subsection{Performance Analysis of MIMO Configuration for UWB GPR}

As mentioned in the beginning of this chapter, MIMO configuration is a promising technique to implement spatial diversity in UWB GPR and to achieve better imaging capability. In this section, we provide performance evaluation of MIMO configuration based on the following aspects: resolution, ISLR, PSLR, SNR, and range of dip-angle. The analysis is based on simulation data generated by GPRMax [86], which is a simulation software based on finitedifference time-domain (FDTD) solution of Maxwell's equations. The performance improvements are verified by systematic comparison between MIMO array and other GPR array configurations, i.e., SISO and SIMO configurations.

\subsubsection{Sensor Configurations}

To conduct fair evaluation on SISO, SIMO, and MIMO configurations, we use a linear array consists of 29 elements that elongates over $x$-axis and moves in the direction of $y$-axis as shown in Fig. 3.1. This array is lifted with height of $10 \mathrm{~cm}$ from the ground surface. The 
distance between each array element is $7.5 \mathrm{~cm}$, while the transmitter and receiver in the same element are collocated.

Both SISO and MIMO arrays comprise 29 pairs of transmitter and receiver, while SIMO array has only 1 transmitter in the middle and 29 receivers. All these arrays utilize Ricker impulse ( $1^{\text {st }}$ Gaussian derivative) as the transmitted waveform with center frequency of 1 GHz. Furthermore, we use 2 versions of MIMO arrays. In the first one, we only use the odd order of transmitters, e.g. $1^{\text {st }}, 3^{\text {rd }}, 5^{\text {th }}$, and so on. Meanwhile, in the second version all the transmitters are employed. We refer the first version of MIMO array as MIMO-half and the second version as MIMO-all. Fig. 3.8 presents all configurations used in this study.
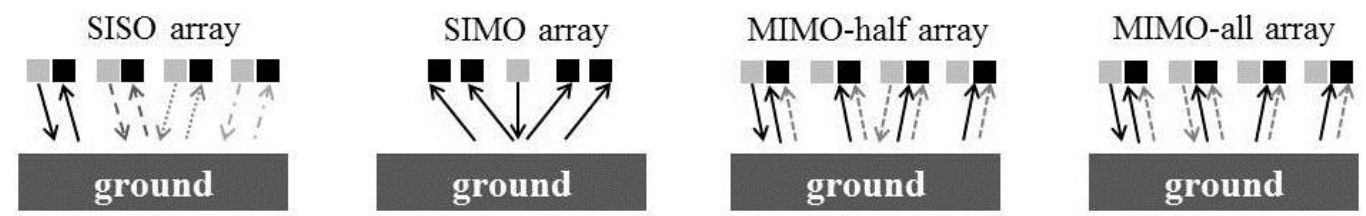

Transmitter
Receiver

Fig. 3.8: SISO, SIMO, MIMO-half, and MIMO-all array configurations.

\subsubsection{Migration Method on SIMO Configuration}

In the investigation of array performance, we decide to use f-k migration as the focusing technique. Unfortunately, f-k migration is not applicable for SIMO configuration since the formulation requires adequate spatial sampling on transmitters and receivers. To overcome this issue, we develop a modified approach of f-k migration that can handle SIMO array. We expect that the modified migration formula has the following form

$$
u_{m}(\mathbf{r})=\int u\left(x_{s}, k_{x g}, \omega\right) A_{1}\left(k_{x g}, \omega, \mathbf{r}\right) e^{-i k R_{s}+i \mathbf{k}_{\mathrm{g}} \cdot \mathbf{r}} d k_{x g} d \omega
$$

In (3.29), $u_{m}(\boldsymbol{r})$ is the migration output, $u\left(x_{s}, k_{x g}, \omega\right)$ is the measurement data of SIMO configuration, $R_{s}$ is the distance from the output point $r$ to the transmitter, $A_{l}$ is the integration kernel, and $\boldsymbol{k}_{g}=\left(k_{x g}, k_{z g}\right)$. The remaining notations are similar to the notation defined in Section 3.1. By comparing (3.29) with (3.20), one can recognize that this equation has similar steps to phase-shift migration, but now we have additional phase factor due to the transmitter position. The next problem is how to determine the kernel $A_{l}$. 
Based on several approximation approaches [103] such as Born's approximation, PhysicalOptic approximation, and Kirchhoff approximation, scattered wave can be expressed in general by (3.30), where $\boldsymbol{r}^{\prime}=\left(x^{\prime}, z^{\prime}\right), R\left(\boldsymbol{r}^{\prime}\right)$ is the reflectivity function of the target, $A_{2}$ is a particular function that depends on the approximation method, $R_{s}$ ' is the distance from $r$ ' to the transmitter, and $R_{g}{ }^{\prime}$ is the distance from $r$ ' to the receiver.

$$
u\left(x_{s}, x_{g}, \omega\right)=\int R\left(\mathbf{r}^{\prime}\right) A_{2}\left(r_{s}, r_{g}, \omega, \mathbf{r}^{\prime}\right) e^{i k\left(R_{s}^{\prime}+R_{g}{ }^{\prime}\right)} d^{2} \mathbf{r}^{\prime}
$$

Since SIMO configuration has regular spatial sampling on receiver side, we can apply Fourier transform over receiver position $x_{g}$ and simplify the result with method of stationary phase, which leads to

$$
u\left(x_{s}, k_{x g}, \omega\right)=\int R\left(\mathbf{r}^{\prime}\right) A_{2}\left(k_{x g}, \omega, \mathbf{r}^{\prime}\right) e^{i k R_{s}^{\prime}-i \mathbf{k}_{\mathrm{g}} \cdot \mathbf{r}^{\prime}} d^{2} \mathbf{r}^{\prime}
$$

In (3.31), the dependency of $A_{2}$ on $x_{s}$ is omitted due to the fixed transmitter location on SIMO array. Substitute the scattered wave model in (3.31) to the migration formula in (3.29), we obtain

$$
u_{m}(\mathbf{r})=\int d^{2} \mathbf{r}^{\prime} R\left(\mathbf{r}^{\prime}\right) \int d k_{x g} d \omega A_{1} A_{2} e^{-i k\left(R_{s}-R_{s}{ }^{\prime}\right)+i \mathbf{k}_{\mathrm{g}} \cdot\left(\mathbf{r}-\mathbf{r}^{\prime}\right)}
$$

To simplify (3.32), we can use high frequency assumption and perform approximation as given in (3.33).

$$
\begin{aligned}
R_{s}{ }^{\prime} & \approx R_{s} \\
k\left(R_{s}{ }^{\prime}-R_{s}\right) & \approx \nabla_{\mathbf{r}^{\prime}}\left[k\left(R_{s}{ }^{\prime}-R_{s}\right)\right]_{\mathbf{r}^{\prime}=\mathbf{r}} \cdot\left(\mathbf{r}^{\prime}-\mathbf{r}\right) \\
& =-\mathbf{k}_{\mathrm{s}} \cdot\left(\mathbf{r}^{\prime}-\mathbf{r}\right)
\end{aligned}
$$

Applying such approximation into (3.32) and changing the integration variable from $\left(k_{x g}, \omega\right)$ into $\boldsymbol{k}=\boldsymbol{k}_{\boldsymbol{s}}+\boldsymbol{k}_{g}=\left(k_{x}, k_{z}\right)$, we get

$$
u_{m}(\mathbf{r})=\int d^{2} \mathbf{r}^{\prime} R\left(\mathbf{r}^{\prime}\right) \int d^{2} \mathbf{k} A_{1} A_{2}|J| e^{i \mathbf{k} \cdot\left(\mathbf{r}-\mathbf{r}^{\prime}\right)}
$$

where $|J|$ is the Jacobian determinant that arises due to the transformation of the integration variables.

Since the objective of migration is to obtain the reflectivity function of the target, we expect that the integration over $\boldsymbol{k}$ in (3.34) yields a delta function. Thus, we select kernel $A_{l}$ to satisfy 


$$
A_{1}=1 /\left(A_{2}|J|\right)
$$

If Born's approximation is utilized for the scattered wave model in (3.30), one can obtain the value of $A_{l}$ as given in (3.36).

$$
A_{1}=\frac{1}{c|k| \sqrt{R_{s}}}\left[k R_{s}-k_{z g} z-k_{x g}\left(x-x_{s}\right)\right]
$$

Fig. 3.9 summarizes all the steps in the proposed migration method. The data is first transformed into frequency wavenumber domain, followed by iteration for every $z$ in the output point. In each iteration, the data is multiplied by interpolation factor $\exp \left(-i k_{z} z\right)$ and converted back into $x$ domain. Then, multiplication by phase factor and kernel $A_{l} \exp \left(-i k R_{s}\right)$ is applied on the data, followed by summation over $\omega$. Overall, these steps are similar to phaseshift migration, except in the multiplication by phase factor.

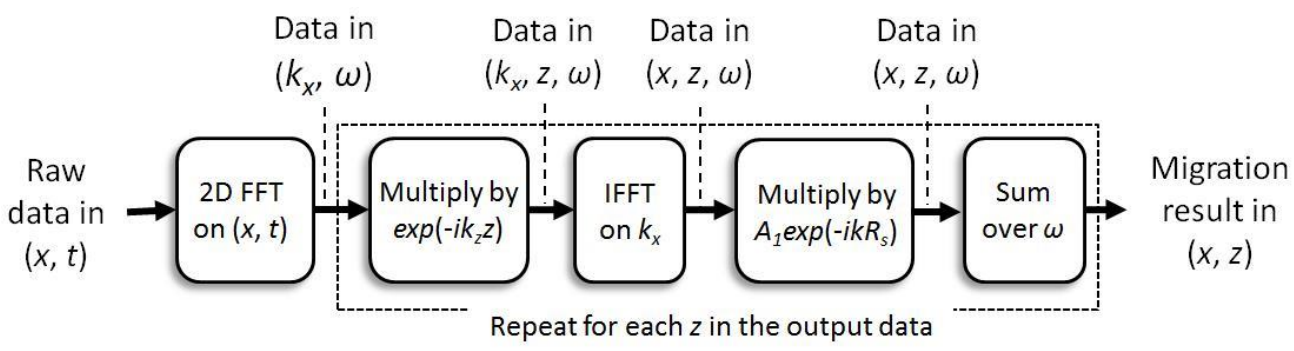

Fig. 3.9: Migration method for SIMO configuration.

To check the proposed algorithm, we generate simulation data of SIMO array on point target in free space. We use the array parameter and transmitted waveform as described in Subsection 3.2.1. Then, we apply Kirchhoff migration [104] and the proposed migration method to the data. The results are given in Fig. 3.10(a) and (b) for Kirchhoff migration and the proposed migration method respectively. We can observe that Kirchhoff migration produces a more focused image with higher side-lobe level and image artifacts.

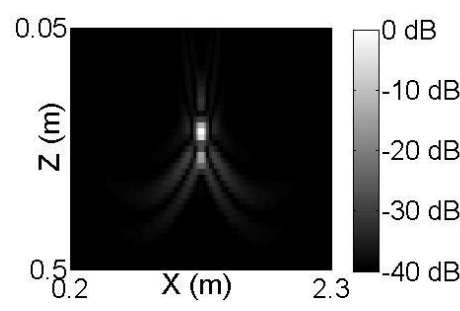

(a) Kirchhoff migration

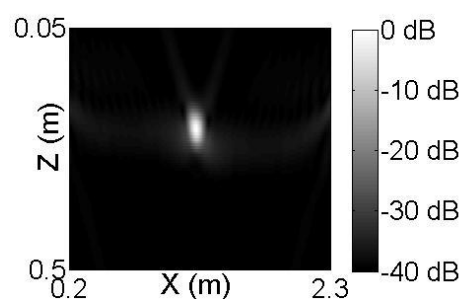

(b) Proposed migration

Fig. 3.10: Results of Kirchhoff migration and the proposed migration method on point target. 


\subsubsection{Resolution, ISLR, and PSLR}

In this subsection, we present the investigation of the following fundamental performance parameters in imaging radar: resolution, ISLR, and PSLR. From the discussion in Section 2.5, we recall that these parameters can be extracted from the PSF of the array. Therefore, we arrange a simulation with a point target placed in the middle of domain as shown in Fig. 3.11. The array lies over $x$-axis so that the cross-range imaging performance is mainly determined by the corresponding array configuration. The subsurface has relative permittivity 6 , relative permeability 1 , and conductivity $0.001 \mathrm{~S} / \mathrm{m}$.

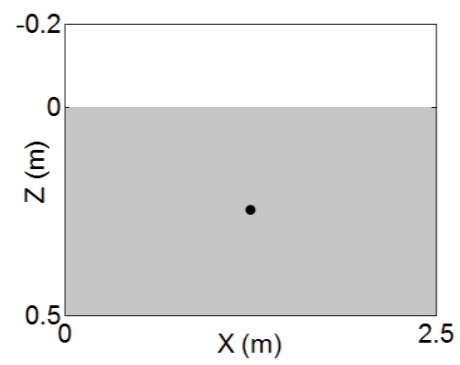

Fig. 3.11: Target configuration to extract PSF.

SISO and MIMO data are processed with f-k migration, while SIMO data is processed with an equivalent phase-shift algorithm developed in Subsection 3.2.2. Fig. 3.12 shows the resulting PSFs of each configuration. In order to observe the resolution and side-lobe level, horizontal slices are taken at the peak point of these PSFs. The results are shown in Fig. 3.13, which are equivalent to the beam-pattern of the arrays. As we can see, MIMO-all configuration, which utilizes all the entire transmitters, provides the best resolution and the lowest side-lobe level. Meanwhile, MIMO-half configuration, which utilizes only half of the available transmitters, has slightly wider peak and higher side-lobe. Generally speaking, both MIMO configurations exhibit better resolution and side-lobe level than SISO and SIMO configurations. SIMO configuration has the lowest resolution, while its side-lobe level is a bit lower than SISO configuration. 


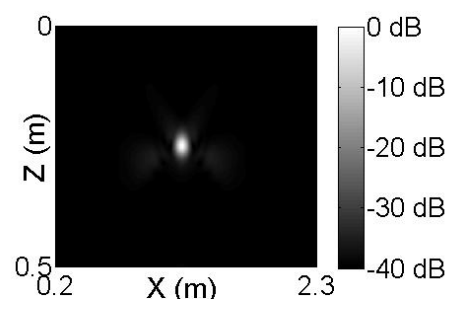

(a) SISO

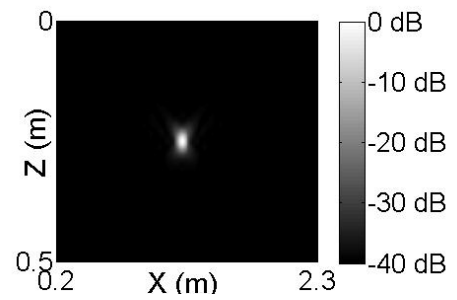

(c) MIMO-half

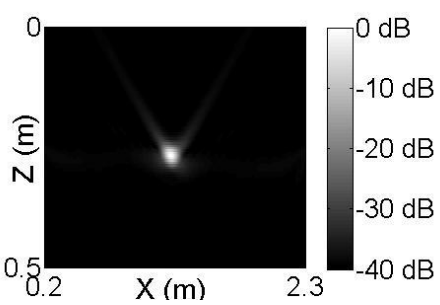

(b) SIMO

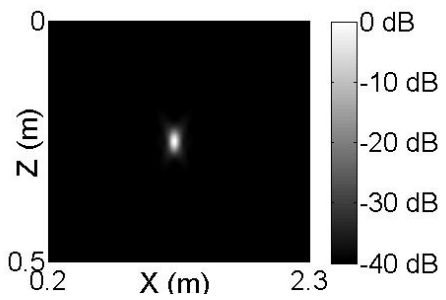

(d) MIMO-all

Fig. 3.12: Point spread function of the 4 array configurations.

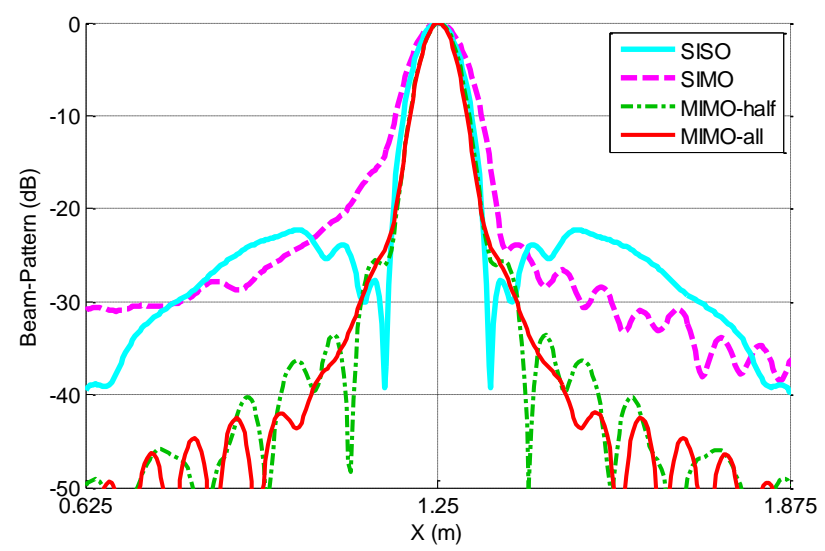

Fig. 3.13: Horizontal slice of the point spread functions.

Furthermore, we examine the performance quantitatively by extracting the value of resolution, ISLR, and PLSR from the beam-patterns, the calculation of which has been described in Section 2.5. The results are given in Table 3.1 and are in agreement with Fig. 3.13.

Table 3.1: PERformance PARAMETERS OF EACH SENSOR CONFIGURATION

\begin{tabular}{|l|c|c|c|c|}
\hline \multicolumn{1}{|c|}{ Parameter } & SISO & SIMO & MIMO-half & MIMO-all \\
\hline Resolution $(\mathrm{m})$ & 0.075 & 0.090 & 0.067 & 0.059 \\
\hline PSLR $(\mathrm{dB})$ & -22 & -24 & -26 & -42 \\
\hline ISLR $(\mathrm{dB})$ & -15 & -17 & -24 & -36 \\
\hline
\end{tabular}

To verify these results, we conduct another simulation that can demonstrate the imaging capabilities of those arrays in real case. The simulation contains 3 pairs of PEC cylinder with 
diameter of $3 \mathrm{~cm}$ and offset of $11 \mathrm{~cm}$ as displayed in Fig. 3.14. This configuration is related to the GPR application for utilities mapping and evaluation of reinforced concrete. The cylinders are placed at various different locations inside the concrete medium, which has relative permittivity 6 , relative permeability 1 , and conductivity $0.001 \mathrm{~S} / \mathrm{m}$.

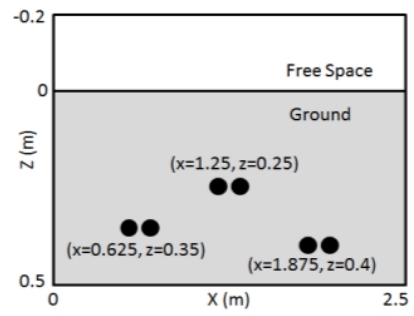

Fig. 3.14: Target configuration with 3 PEC cylinders.

The results of this simulation are given in Fig. 3.15. It can be clearly observed that both MIMO configurations improve horizontal resolution and side-lobe level comparing with the SISO and SIMO configurations. The two closely located cylinders can be distinguished in MIMO images. By contrast, these cylinders appear to be overlapped in SISO image, while SIMO image shows imbalance reflection between the cylinders near the center and the cylinders close to the edge. Moreover, MIMO arrays also exhibit cleaner image and less artifacts, which support the advantages of MIMO on side-lobe suppression.

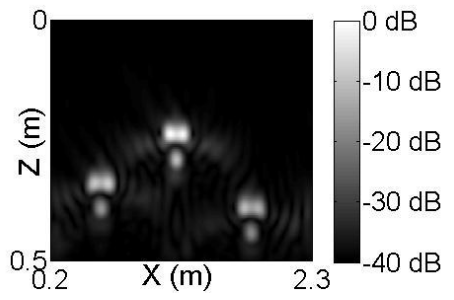

(a) SISO

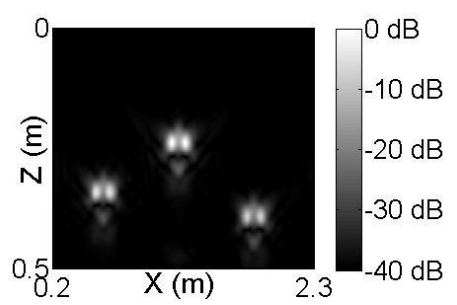

(c) MIMO-half

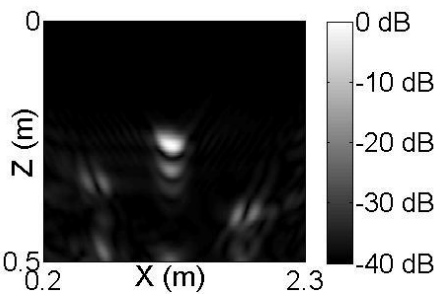

(b) SIMO

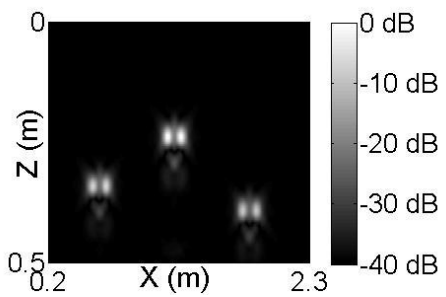

(d) MIMO-all

Fig. 3.15: Results of simulation with 3 PEC cylinders. 


\subsubsection{SNR Improvement}

The simulations that have been carried out in previous results are based on ideal condition where noise components are neglected. In practice, such components along with other irregularities always exist and cause degradation in the final image. MIMO array, which has higher number of measurement, could potentially increase the reflected power from the desired target and thereby enhance the SNR. In this subsection, we present evaluation on imaging performance in which the noise components are taken into account.

We investigate the noise effects by analyzing the imaging performance over thin void, which is related to infrastructure survey application. The simulation configuration is given in Fig. 3.16. The target is a thin rectangular gap with fixed horizontal size of $20 \mathrm{~cm}$. We take several values of the vertical thickness of the void $(d z): 0.2 \mathrm{~cm}, 0.5 \mathrm{~cm}$, and $1 \mathrm{~cm}$. The subsurface medium has relative permittivity 6 , relative permeability 1 , and conductivity $0.001 \mathrm{~S} / \mathrm{m}$. The AWGN noise is also added to the simulation data with the same power for each sensor configuration.

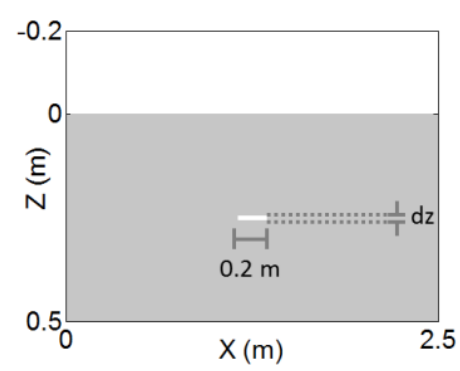

Fig. 3.16: Target configuration with thin void.

The results of this simulation are provided in Fig. 3.17 to Fig. 3.19 for void thickness of 0.2 $\mathrm{cm}, 0.5 \mathrm{~cm}$, and $1 \mathrm{~cm}$ respectively. The results clearly show that the reflections from void with thickness of $0.2 \mathrm{~cm}$ are mostly obscured by noise for all arrays. Meanwhile, all the arrays can resolve the void with vertical size $0.5 \mathrm{~cm}$ and $1 \mathrm{~cm}$. However, the MIMO-half and MIMO-all arrays give clearer target reflection compared to the SISO and SIMO arrays. These results imply that MIMO arrays are able to provide higher SNR, and thus can improve the detection capability on small target. 


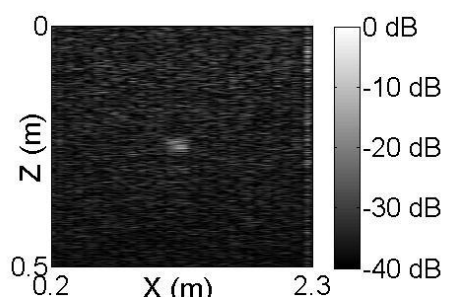

(a) SISO

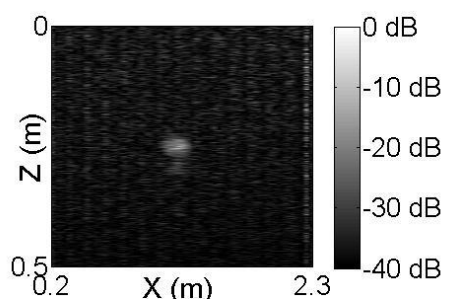

(c) MIMO-half

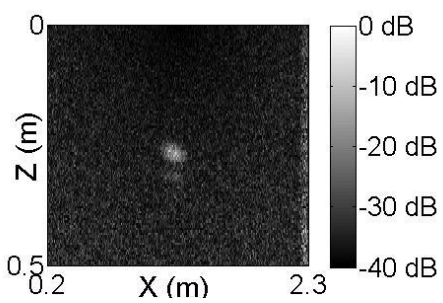

(b) SIMO

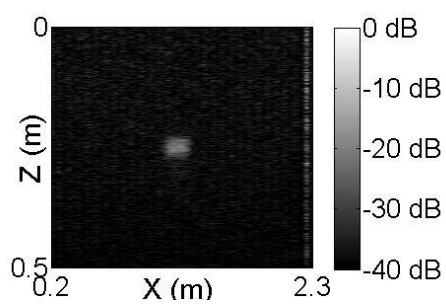

(d) MIMO-all

Fig. 3.17: Simulation results on void with thickness of $0.2 \mathrm{~cm}$.

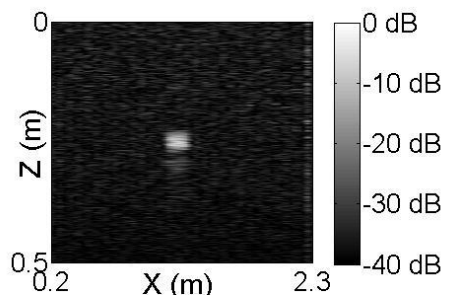

(a) SISO

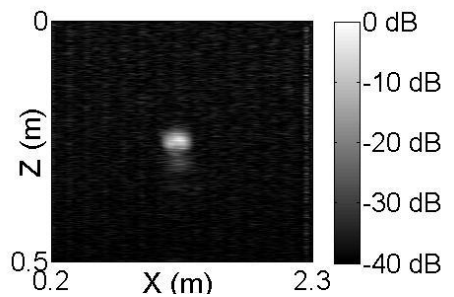

(c) MIMO-half

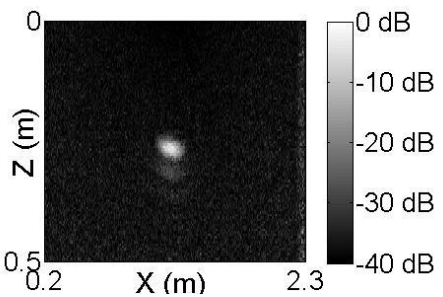

(b) SIMO

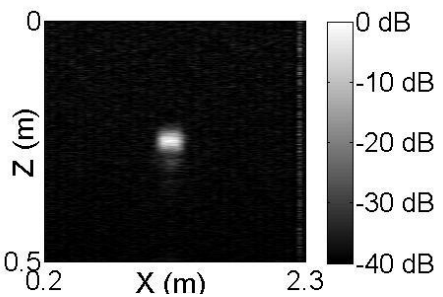

(d) MIMO-all

Fig. 3.18: Simulation results on void with thickness of $0.5 \mathrm{~cm}$. 


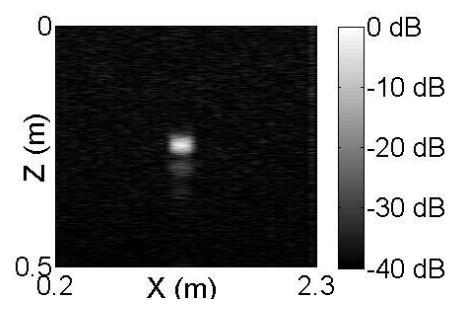

(a) SISO

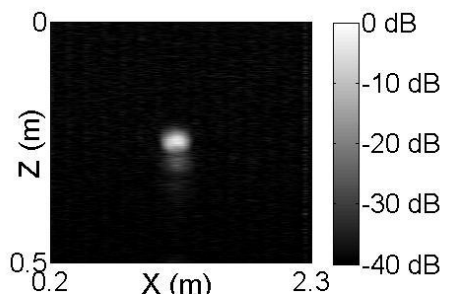

(c) MIMO-half

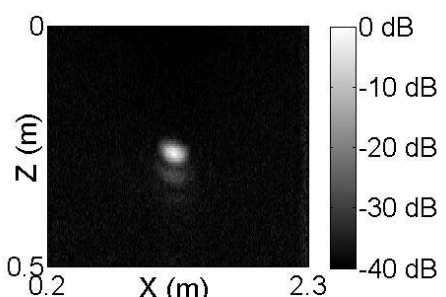

(b) SIMO

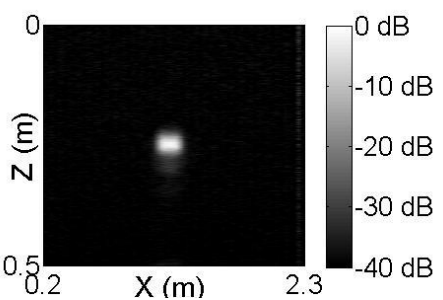

(d) MIMO-all

Fig. 3.19: Simulation results on void with thickness of $1 \mathrm{~cm}$.

\subsubsection{Range of Dip-Angle}

The last aspect to be considered in this section is the range of dip-angle. We recall from Section 2.5 that range of dip-angle indicates the maximum inclination angle of a surface that still can be resolved by the radar. According to Snell's law, the incident angle $\left(\theta_{i}\right)$ must be equal to the reflection angle $\left(\theta_{r}\right)$ in the scattering process. Such principle implies that a reflection on a particular surface can occur only if the sum of incident vector $\hat{\mathbf{n}}_{\mathrm{s}}$ and reflection vector $\hat{\mathbf{n}}_{\mathrm{g}}$ is aligned with the normal vector $\hat{\mathbf{n}}$ of the surface, as formulated in (3.37). This process can be illustrated by Fig. 3.20. Practically, the array dimension, the transmitter and receiver configuration, and the antenna beam-width will confine the maximum incident and reflection angles in the measurement, and thereby limit the range of tilted surface that can be imaged.

$$
\hat{\mathbf{n}}=\frac{\hat{\mathbf{n}}_{\mathrm{s}}+\hat{\mathbf{n}}_{\mathrm{g}}}{\left|\hat{\mathbf{n}}_{\mathrm{s}}+\hat{\mathbf{n}}_{\mathrm{g}}\right|}
$$




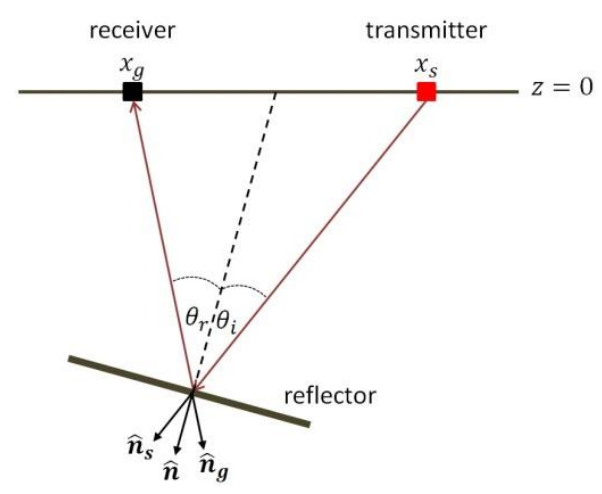

Fig. 3.20: Illustration of scattering process by a dipping reflector.

MIMO configuration permits any transmitter-receiver combination within the array, which in turn enables wider range of incident and reflection angles compared to SISO and SIMO configurations. Therefore, we can expect that MIMO array potentially offers better performance in imaging a tilted surface.

To investigate the capability of each array in imaging a dipping surface, we conduct a simulation on void targets with shapes given in Fig. 3.21. We simulate these targets with the following values of $\theta: 120^{\circ}, 135^{\circ}$, and $150^{\circ}$. The subsurface medium has relative permittivity 6 , relative permeability 1 , and conductivity $0.001 \mathrm{~S} / \mathrm{m}$

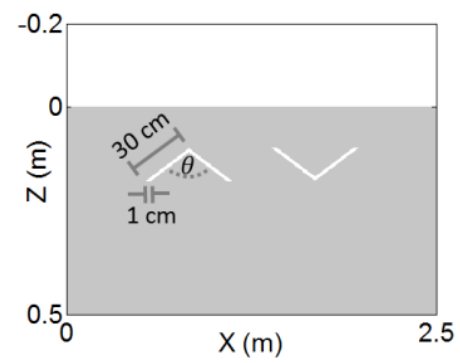

Fig. 3.21: Target configuration with 2 voids.

The simulation results are presented in Fig. 3.22 to Fig. 3.24 for $\theta=120^{\circ}, \theta=135^{\circ}$, and $\theta=$ $150^{\circ}$ respectively. For $\theta=120^{\circ}$, all the arrays are unable to resolve the dipping parts of the voids. For $\theta=135^{\circ}$, SISO array shows some reflection from the dipping parts of the voids. However, this reflection is misplaced and some artifacts also appear in the image. SIMO array only resolves one dipping side of each void. This unbalance reflection occurs due to the location of transmitter and receivers in SIMO array that makes those surfaces reflect the wave better than other surfaces. MIMO-all array exhibits reflection slightly on the tilted surfaces of the voids. Although MIMO-half array does not give clear image on the dipping surfaces, it 
still has less image artifacts compared to the SISO array. Finally, for $\theta=150^{\circ}$, all the arrays can resolve the voids. The shapes of the dipping part remain to be distorted in the SISO image, while the SIMO array can only resolve one side of each void. By contrast, both MIMO arrays can resolve the dipping surfaces correctly. These results confirm our prediction of MIMO capability in improving range of dip-angle.

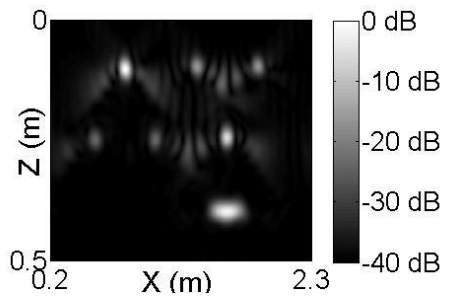

(a) SISO

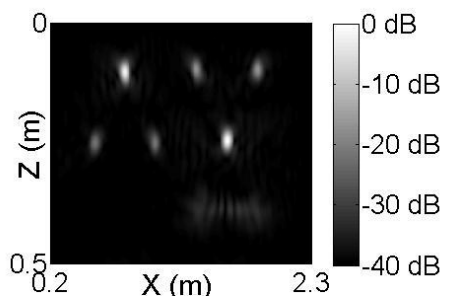

(c) MIMO-half

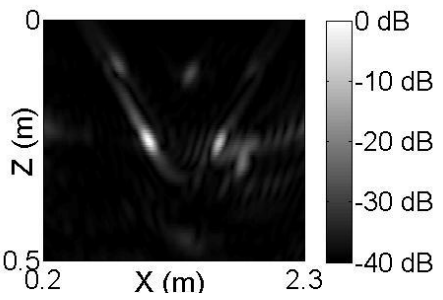

(b) SIMO

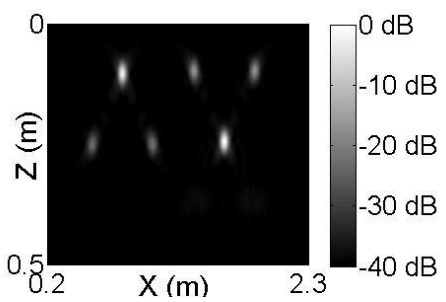

(d) MIMO-all

Fig. 3.22: Simulation results on 2 voids with $\theta=120^{\circ}$.

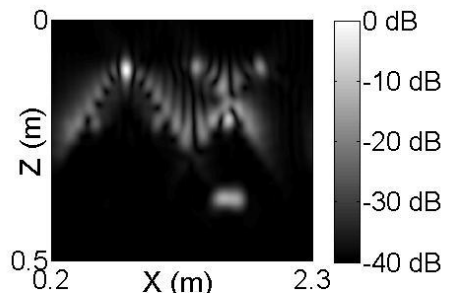

(a) SISO

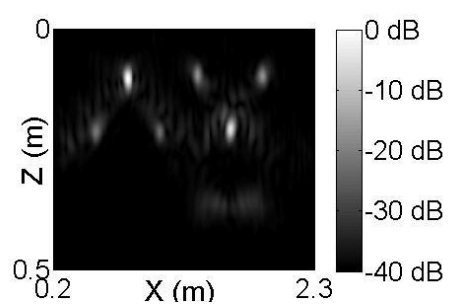

(c) MIMO-half

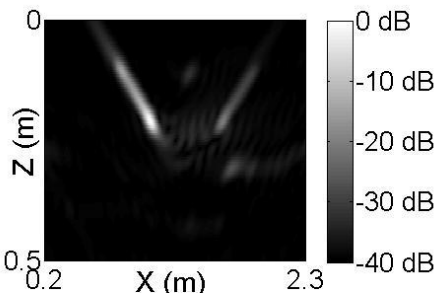

(b) SIMO

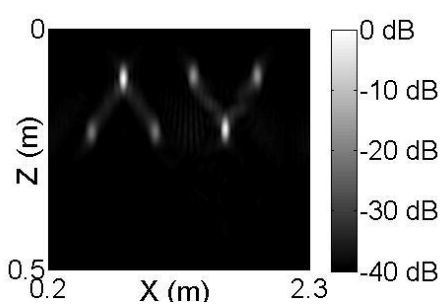

(d) MIMO-all

Fig. 3.23: Simulation results on 2 voids with $\theta=135^{\circ}$. 


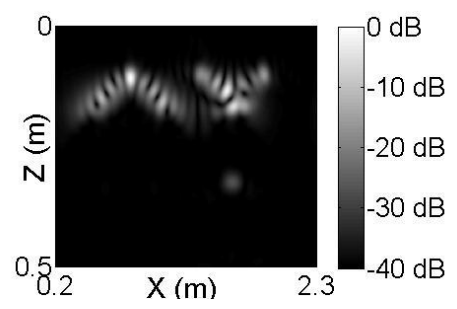

(a) $\mathrm{SISO}$

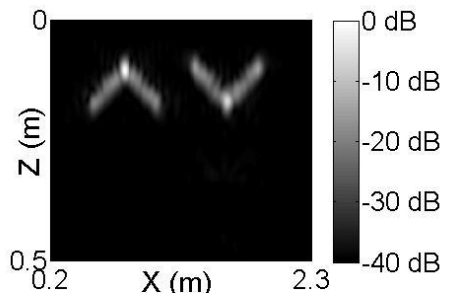

(c) MIMO-half

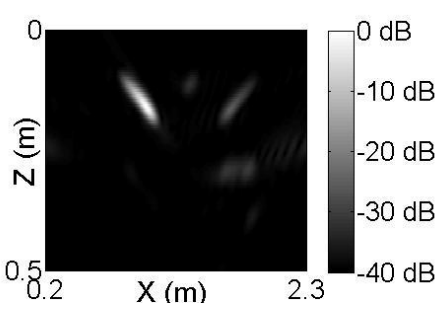

(b) SIMO

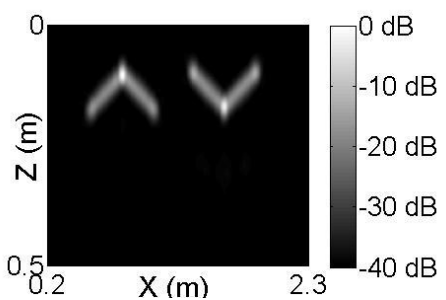

(d) MIMO-all

Fig. 3.24: Simulation results on 2 voids with $\theta=150^{\circ}$.

\subsubsection{Theoretical Analysis of Array Performance}

In the previous subsections, we have presented the performance analysis on SISO, SIMO, and MIMO configurations based on simulation result. In general, we can summarize the following advantages provided by MIMO configuration: resolution improvement, reduction of side-lobe level, higher SNR, and wider dip-angle range. However, simulation result is not sufficient to take general conclusion on the performance improvement provided by MIMO configuration. Moreover, an analytical formulation of MIMO array performance is necessary to control the trade-off between measurement complexity and imaging capability. Thus, to support our previous simulation result, in this subsection we present theoretical analysis on the array performance. We focus on the following 3 aspects: resolution, SNR, and resolving capability over dipping surface.

Before going into detailed analysis on each performance aspect, we need to obtain the formulation of received signal and migration result on each configuration. Suppose that we have a transmitter located at $\left(x_{s}, 0\right)$. This transmitter emits impulse waveform signal whose spectrum is denoted by $F(\omega)$. The transmitted wave propagates into the subsurface and is scattered by target with scattering potential denoted by $V\left(\boldsymbol{x}^{\prime}\right)$. The scattered wave is then received by the receiver located at $\left(x_{g}, 0\right)$. Based on Born's approximation, the received signal can be formulated as in (3.38), where $u^{(i n)}\left(\boldsymbol{x}^{\prime}, x_{s}, \omega\right)$ is the transmitted signal as given in (3.39), $G\left(x_{g}, x^{\prime}, \omega\right)$ is a Green's function as given in (3.40), $R_{s}$ ' is the distance from the transmitter at $\left(x_{s}, 0\right)$ to $\left(x^{\prime}, z^{\prime}\right)$, and $R_{g}{ }^{\prime}$ is the distance from receiver at $\left(x_{g}, 0\right)$ to $\left(x^{\prime}, z^{\prime}\right)$. 


$$
\begin{gathered}
u^{(s)}\left(x_{s}, x_{g}, \omega\right)=\int V\left(\mathbf{x}^{\prime}\right) u^{(i n)}\left(\mathbf{x}^{\prime}, x_{s}, \omega\right) G\left(x_{g}, \mathbf{x}^{\prime}, \omega\right) d^{2} \mathbf{x}^{\prime} \\
u^{(i n)}\left(\mathbf{x}^{\prime}, x_{s}, \omega\right)=\frac{F(\omega) e^{i k R s^{\prime}}}{4 \pi R_{s}{ }^{\prime}} \\
G\left(x_{g}, \mathbf{x}^{\prime}, \omega\right)=\frac{e^{i k R_{g}{ }^{\prime}}}{4 \pi R_{g}{ }^{\prime}}
\end{gathered}
$$

\subsubsection{Formulation of Migration Result on SISO Configuration}

For SISO configuration, the transmitter and the receiver are collocated, so we have $x_{s}=x_{g}=x$, and (3.38) can be simplified into (3.41). In (3.41), we have additional rectangular function rect due to the limitation of array length, where $x_{m}$ is the mid-point of the array and $L$ is the array length.

$$
u^{(s)}(x, \omega)=\int V\left(\mathbf{x}^{\prime}\right) F(\omega) \frac{e^{i 2 k R^{\prime}}}{16 \pi^{2} R^{\prime 2}} \operatorname{rect}\left(\frac{x-x_{m}}{L}\right) d^{2} \mathbf{x}^{\prime}
$$

The f-k migration algorithm begins with Fourier transform of the received data over $x$ and $t$. We can apply such transformation to (3.41) and simplify the result by using method of stationary phase (MOSP). The corresponding stationary phase is given by (3.42).

$$
\Phi=\frac{2 \omega R}{c}-k_{x} x
$$

By setting the first derivative of (3.42) to be zero and solve the equation with respect to $x$, we can get the stationary point at

$$
x=x^{\prime}-\frac{k_{x}}{k_{z}} z^{\prime}
$$

with $k_{z}=-2 k \sqrt{1-\left(\frac{k_{x}}{2 k}\right)^{2}}$.

The resulting phase at stationary point is given by (3.44).

$$
\Phi=-k_{x} x^{\prime}-k_{z} z^{\prime}
$$

Finally, the resulting Fourier transform formula is given by (3.45). 


$$
u^{(s)}\left(k_{x}, \omega\right)=\int A_{1} V\left(\mathbf{x}^{\prime}\right) F(\omega) \operatorname{rect}\left(\frac{\left(k_{x} / k_{z}\right)-\left(x-x_{m}\right) / z^{\prime}}{L / z^{\prime}}\right) \operatorname{rect}\left(\frac{k_{x}}{2 \pi / d x}\right) e^{-i k_{x} x^{\prime}-i k_{z} z^{\prime}} d^{2} \mathbf{x}^{\prime}
$$

We can see that there are 3 main factors that confine the integration range over $\left(k_{x}, k_{z}\right)$. The first factor is $F(\omega)$, which limit the wavenumber range as given in (3.46).

$$
\frac{2 \omega_{\min }}{c} \leq \sqrt{k_{x}^{2}+k_{z}^{2}} \leq \frac{2 \omega_{\max }}{c}
$$

The second factor is the first rectangular function in (3.45), which confine the wavenumber range as in (3.47).

$$
-\frac{L_{x}}{2 z^{\prime}}+\frac{x^{\prime}-x_{m}}{z^{\prime}} \leq \frac{k_{x}}{k_{z}} \leq \frac{L_{x}}{2 z^{\prime}}+\frac{x^{\prime}-x_{m}}{z^{\prime}}
$$

Finally, the last factor is the second rectangular function in (3.45) that limit the wavenumber range as in (3.48).

$$
-\frac{\pi}{d x} \leq k_{x} \leq \frac{\pi}{d x}
$$

\subsubsection{Formulation of Migration Result on SIMO Configuration}

For SIMO configuration, the received signal is given by (3.49), where $x_{s}$ is a constant for this case.

$$
u^{(s)}\left(x_{g}, \omega\right)=\int V\left(\mathbf{x}^{\prime}\right) F(\omega) \frac{e^{i k\left(R_{s}{ }^{\prime}+R_{g}{ }^{\prime}\right)}}{16 \pi^{2} R_{s}{ }^{\prime} R_{g}{ }^{\prime}} r e c t\left(\frac{x_{g}-x_{m}}{L}\right) d^{2} \mathbf{x}^{\prime}
$$

Similar to the SISO configuration, the migration algorithm for SIMO configuration starts with Fourier transform over $x$ and $t$. We can apply Fourier transform on (3.49) and simplify the result by using MOSP with stationary phase given in (3.50).

$$
\Phi=\frac{\omega R_{g}}{c}-k_{x g} x_{g}
$$

By set the derivative of (3.50) to zero and solve such equation with respect to $x_{g}$, we can obtain the stationary point as given in (3.51).

$$
x_{g}=x^{\prime}-\frac{k_{x g}}{k_{z g}} z^{\prime}
$$


with $k_{z g}=-k \sqrt{1-\left(\frac{k_{x g}}{k}\right)^{2}}$.

Substitute (3.51) to the phase term in (3.50), we obtain the corresponding phase at the stationary point as

$$
\Phi=-k_{x g} x^{\prime}-k_{z g} z^{\prime}
$$

The MOSP approximation result of the integration in (3.49) is thus given by

$$
u^{(s)}\left(k_{x g}, \omega\right)=\int A_{1} V\left(\mathbf{x}^{\prime}\right) F(\omega) \operatorname{rect}\left(\frac{\left(k_{x g} / k_{z g}\right)-\left(x^{\prime}-x_{m}\right) / z^{\prime}}{L / z^{\prime}}\right) \operatorname{rect}\left(\frac{k_{x g}}{2 \pi / d x}\right) e^{i k R_{s}{ }^{\prime}-i k_{x g} x^{\prime}-i k_{z g} z^{\prime}} d^{2} \mathbf{x}^{\prime}
$$

From (3.53), we can see that there are 3 main factors that confine the wavenumber range over $\left(k_{x}, k_{z}\right)$. The first factor is $F(\omega)$, which limits the frequency domain within certain range as given in (3.54).

$$
\omega_{\min } \leq \omega \leq \omega_{\max }
$$

The second factor is the first rectangular function in (3.53) which limits the wavenumber range as given in (3.55).

$$
-\frac{L_{x}}{2 z^{\prime}}+\frac{x^{\prime}-x_{m}}{z^{\prime}} \leq \frac{k_{x g}}{k_{z g}} \leq \frac{L_{x}}{2 z^{\prime}}+\frac{x^{\prime}-x_{m}}{z^{\prime}}
$$

The last factor is the second rectangular function in (3.53) that confines the wavenumber range as given in (3.56).

$$
-\frac{\pi}{d x} \leq k_{x g} \leq \frac{\pi}{d x}
$$

The 3 factors in (3.54) to (3.56) are given in term of $\omega, k_{x g}$, and $k_{z g}$. Meanwhile, we are interested in analyzing the wavenumber domain over $\left(k_{x}, k_{z}\right)$. Therefore, we need to transform $\omega, k_{x g}$, and $k_{z g}$ as function of $k_{x}$ and $k_{z}$. We can resolve thus shortcomings by substituting (3.53) into the migration formula (3.29) as given in (3.57).

$$
u_{m}(\mathbf{x})=\int A_{2} V\left(\mathbf{x}^{\prime}\right) F(\omega) \operatorname{rect}\left(\frac{\left(k_{x g} / k_{z g}\right)-\left(x^{\prime}-x_{m}\right) / z^{\prime}}{L / z^{\prime}}\right) \operatorname{rect}\left(\frac{k_{x g}}{2 \pi / d x}\right) e^{i k\left(R_{s}^{\prime}-R_{s}\right)-i \mathbf{k}_{\mathbf{g}} \cdot\left(\mathbf{x}^{\prime} \mathbf{x}\right)} d^{2} \mathbf{x}^{\prime} d k_{x g} d \omega
$$


Then, we simplify the phase by using high frequency approximation as given in (3.58).

$$
\begin{aligned}
k\left(R_{s}{ }^{\prime}-R_{s}\right) & \left.\approx k \nabla_{\mathbf{x}^{\prime}}\left(R_{s}{ }^{\prime}-R_{s}\right)\right|_{\mathbf{x}^{\prime}=\mathbf{x}} \cdot\left(\mathbf{x}^{\prime}-\mathbf{x}\right) \\
& =-\mathbf{k}_{\mathbf{x s}} \cdot\left(\mathbf{x}^{\prime}-\mathbf{x}\right)
\end{aligned}
$$

The total wavenumber in this case is given by (3.59).

$$
\begin{aligned}
& k_{x}=k_{x g}-k \frac{\left(x-x_{s}\right)}{R_{s}} \\
& k_{z}=k_{z g}-k \frac{z}{R_{s}}
\end{aligned}
$$

We can solve (3.59) and obtain $k, k_{x g}$, and $k_{z g}$ as function of $k_{x}$ and $k_{z}$ as given in (3.60), (3.61), and (3.62) respectively. Thus, for a given point $\left(k_{x}, k_{z}\right)$ in wavenumber domain, we can calculate the corresponding $k, k_{x g}$, and $k_{z g}$, and obtain the wavenumber range as given in (3.54) to (3.56).

$$
\begin{gathered}
k=-\frac{R_{s}\left(k_{x}^{2}+k_{z}^{2}\right)}{2\left[k_{x}\left(x-x_{s}\right)+k_{z} z\right]} \\
k_{x g}=k_{x}+k \frac{\left(x-x_{s}\right)}{R_{s}} \\
k_{z g}=k_{z}+k \frac{z}{R_{s}}
\end{gathered}
$$

\subsubsection{Formulation of Migration Result on MIMO Configuration}

For MIMO configuration, the received signal is given by (3.63).

$$
u^{(s)}\left(x_{s}, x_{g}, \omega\right)=\int V\left(\mathbf{x}^{\prime}\right) F(\omega) \frac{e^{i k\left(R_{s}{ }^{\prime}+R_{g}{ }^{\prime}\right)}}{16 \pi^{2} R_{s}{ }^{\prime} R_{g}{ }^{\prime}} \operatorname{rect}\left(\frac{x_{s}-x_{m}}{L}\right) \operatorname{rect}\left(\frac{x_{g}-x_{m}}{L}\right) d^{2} \mathbf{x}^{\prime}
$$

In this equation, we have full flexibility on the value of $x_{s}$ and $x_{g}$. Similar to all the previous cases, we start with Fourier transform on (3.63) and simplify the equation by using MOSP. The corresponding stationary phase is given by (3.64).

$$
\Phi=\frac{\omega\left(R_{s}+R_{g}\right)}{c}-k_{x g} x_{g}-k_{x s} x_{s}
$$


By taking the first derivative of (3.64) with respect to $x_{s}$ and $x_{g}$, and set them to zero, we obtain the stationary point as given in (3.65), where $k_{z s}$ and $k_{z g}$ are given in (3.66).

$$
\begin{gathered}
x_{s}=x^{\prime}-\frac{k_{x s}}{k_{z s}} z^{\prime} \\
x_{g}=x^{\prime}-\frac{k_{x g}}{k_{z g}} z^{\prime} \\
k_{z s}=-k \sqrt{1-\left(\frac{k_{x s}}{k}\right)^{2}} \\
k_{z g}=-k \sqrt{1-\left(\frac{k_{x g}}{k}\right)^{2}}
\end{gathered}
$$

The corresponding stationary phase is given by (3.67).

$$
\Phi=-\left(k_{x s}+k_{x g}\right) x^{\prime}-\left(k_{z s}+k_{z g}\right) z^{\prime}
$$

The integration result after MOSP is given by (3.68).

$$
\begin{aligned}
u^{(s)}\left(k_{x s}, k_{x g}, \omega\right)= & \int A_{1} V\left(\mathbf{x}^{\prime}\right) F(\omega) \operatorname{rect}\left(\frac{\left(k_{x s} / k_{z s}\right)-\left(x^{\prime}-x_{m}\right) / z^{\prime}}{L / z^{\prime}}\right) \operatorname{rect}\left(\frac{\left(k_{x g} / k_{z g}\right)-\left(x^{\prime}-x_{m}\right) / z^{\prime}}{L / z^{\prime}}\right) \\
& \operatorname{rect}\left(\frac{k_{x s}}{2 \pi / d x}\right) \operatorname{rect}\left(\frac{k_{x g}}{2 \pi / d x}\right) e^{-i\left(k_{x s}+k_{x g}\right) x^{\prime}-i\left(k_{z s}+k_{z g}\right) z^{\prime}} d^{2} \mathbf{x}^{\prime}
\end{aligned}
$$

Similar as before, we can see that there are 3 main factors that confine the wavenumber range in (3.68). The first factor is $F(\omega)$, the second factor is the first rectangular function, while the last factor is second next rectangular function, each of which confines the wavenumber range as given in (3.69), (3.70), and (3.71) respectively.

$$
\begin{gathered}
\frac{2 \omega_{\min } \cos \gamma}{c} \leq \sqrt{k_{x}^{2}+k_{z}^{2}} \leq \frac{2 \omega_{\max } \cos \gamma}{c} \text { with } \tan \gamma=\frac{k_{h}}{k_{z}} \\
-\frac{L_{x}}{2 z^{\prime}}+\frac{x^{\prime}-x_{m}}{z^{\prime}} \leq \frac{k_{x s}}{k_{z s}} \leq \frac{L_{x}}{2 z^{\prime}}+\frac{x^{\prime}-x_{m}}{z^{\prime}} \quad \text { and } \quad-\frac{L_{x}}{2 z^{\prime}}+\frac{x^{\prime}-x_{m}}{z^{\prime}} \leq \frac{k_{x g}}{k_{z g}} \leq \frac{L_{x}}{2 z^{\prime}}+\frac{x^{\prime}-x_{m}}{z^{\prime}} \\
-\frac{\pi}{d x} \leq k_{x g} \leq \frac{\pi}{d x} \quad \text { and } \quad-\frac{\pi}{d x} \leq k_{x g} \leq \frac{\pi}{d x}
\end{gathered}
$$

The wavenumber ranges in (3.70) and (3.71) are given in terms of $\omega, k_{z s}$, and $k_{z g}$. Meanwhile, we are interested in analyzing the wavenumber range over $k_{x s}, k_{x g}$, and $k_{z}$. Thus, we need to define $\omega, k_{z s}$, and $k_{z g}$ as function of $k_{x s}, k_{x g}$, and $k_{z}$. We can solve (3.66) and obtain $\omega$ as 
function of $k_{x s}, k_{x g}$, and $k_{z}$ as given in (3.72). Then, we can use the value of $\omega$ and (3.66) to obtain $k_{z s}$ and $k_{z g}$.

$$
\omega=-\frac{k_{z} c}{2} \sqrt{\left(1+\frac{k_{m}^{2}}{k_{z}^{2}}\right)\left(1+\frac{k_{h}^{2}}{k_{z}^{2}}\right)}
$$

\subsubsection{Analysis on Resolution}

In the following part, we will analyze the resulting resolution on each configuration. We have derived the necessary formulation of migration results for each array as given in (3.45) for SISO configuration, (3.53) for SIMO configuration, and (3.68) for MIMO configuration. In general, these expressions imply that the migration result will be the filtered version of the target scattering potential $V\left(x^{\prime}\right)$ over a limited wavenumber range $D_{k}$. Thus, the resolution of the imaging result is mainly determined by the integration range $D_{k}$. Such wavenumber ranges have been derived in (3.46) to (3.48) for SISO array, (3.54) to (3.56) for SIMO array, and (3.69) to (3.71) for MIMO array. By using our array specification as described in Subsection 3.2.1, we can obtain the integration range of each configuration as presented in Fig. 3.25.

(a) SISO

(b) SIMO

(c) MIMO-1

(d) MIMO-2
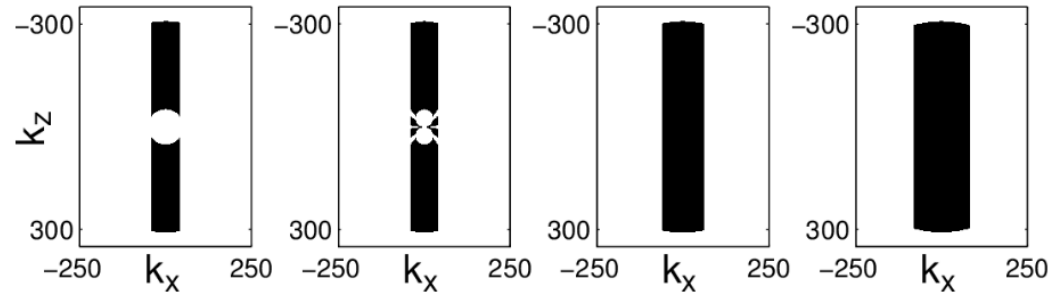

Fig. 3.25: Integration range of each array in the wavenumber domain $\left(k_{x}, k_{z}\right)$.

From Fig. 3.25, we can see that MIMO configuration has broader horizontal wavenumber range than SISO and SIMO, while the vertical wavenumber range of all these configurations are almost equal. Since the migration result is the inverse Fourier transform of target scattering potential $V\left(\boldsymbol{x}^{\prime}\right)$ over these range, the broader range implies higher resolution. Therefore, we can conclude that MIMO configuration will yield higher horizontal resolution than SISO and SIMO arrays. 


\subsubsection{Analysis on SNR}

In this section, we present analysis on SNR for the three sensor configurations. For the object of analysis, we will use a point target with scattering potential given in (3.73), where $x_{0}$ and $z_{0}$ are the horizontal and vertical locations of the target.

$$
V\left(\mathbf{x}^{\prime}\right)=k^{2} \delta\left(x^{\prime}-x_{0}\right) \delta\left(z^{\prime}-z_{0}\right)
$$

Substitute (3.73) into the received signal of each array, we can obtain (3.74) to (3.76) for SISO, SIMO, and MIMO arrays respectively, where $R$ is the distance from $(x, 0)$ to $\left(x_{0}, z_{0}\right), R_{s}$ is the distance from $\left(x_{s}, 0\right)$ to $\left(x_{0}, z_{0}\right)$, and $R_{g}$ is the distance from $\left(x_{g}, 0\right)$ to $\left(x_{0}, z_{0}\right)$.

$$
\begin{gathered}
u^{(s)}(x, \omega)=k^{2} F(\omega) \frac{e^{i 2 k R}}{16 \pi^{2} R^{2}} \\
u^{(s)}\left(x_{g}, \omega\right)=k^{2} F(\omega) \frac{e^{i 2 k\left(R_{s}+R_{g}\right)}}{16 \pi^{2} R_{s} R_{g}} \\
u^{(s)}\left(x_{s}, x_{g}, \omega\right)=k^{2} F(\omega) \frac{e^{i k\left(R_{s}+R_{g}\right)}}{16 \pi^{2} R_{s} R_{g}}
\end{gathered}
$$

By omitting the kernel term, we can approximate those equations in time domain as (3.77) to (3.79) for SISO, SIMO, and MIMO arrays respectively. We also add the noise term $n(t)$, which is an i.i.d white Gaussian noise with zero mean and variance $\sigma^{2}$.

$$
\begin{gathered}
u^{(s)}(x, \omega)=\frac{\delta(t-2 R / c)}{16 \pi^{2} R^{2}}+n(t) \\
u^{(s)}\left(x_{g}, \omega\right)=\frac{\delta\left(t-\left(R_{s}+R_{g}\right) / c\right)}{16 \pi^{2} R_{s} R_{g}}+n(t) \\
u^{(s)}\left(x_{s}, x_{g}, \omega\right)=\frac{\delta\left(t-\left(R_{s}+R_{g}\right) / c\right)}{16 \pi^{2} R_{s} R_{g}}+n(t)
\end{gathered}
$$

For the sake of simplicity, we take diffraction stack algorithm as focusing method to analyze the SNR, the formula of which is given in (3.80), with $X$ denotes the available sample of $x_{s}$ and $x_{g}, t_{0}=2 R / c$ for SISO, and $t_{0}=\left(R_{s}+R_{g}\right) / c$ for SIMO and MIMO.

$$
u\left(x_{0}, z_{0}\right)=\sum_{X} u^{(s)}\left(x_{s}, x_{g}, t=t_{0}\right)
$$


Substitute (3.77) to (3.79) into such migration formula, we obtain (3.81) to (3.83) respectively.

$$
\begin{gathered}
u\left(x_{0}, z_{0}\right)=\sum_{x} \frac{1}{16 \pi^{2} R^{2}}+\sum_{x} n\left(t=t_{0}\right) \\
u\left(x_{0}, z_{0}\right)=\sum_{x_{g}} \frac{1}{16 \pi^{2} R_{s} R_{g}}+\sum_{x_{g}} n\left(t=t_{0}\right) \\
u\left(x_{0}, z_{0}\right)=\sum_{x_{s}, x_{g}} \frac{1}{16 \pi^{2} R_{s} R_{g}}+\sum_{x_{s}, x_{g}} n\left(t=t_{0}\right)
\end{gathered}
$$

We can see that the first term on the right hand side is the signal component, while the second term is the noise component. Finally, we can formulate the SNR as in (3.84) to (3.86) for SISO, SIMO, and MIMO arrays respectively.

$$
\begin{aligned}
& \operatorname{SNR}_{\text {SISO }}=\frac{\left(\sum_{x} 1 /\left(16 \pi^{2} R^{2}\right)\right)^{2}}{E\left[\left(\sum_{x} n\left(t=t_{0}\right)\right)^{2}\right]}=\frac{\left(\sum_{x} 1 /\left(16 \pi^{2} R^{2}\right)\right)^{2}}{N_{x} \sigma^{2}} \\
& \operatorname{SNR}_{\text {SIMO }}=\frac{\left(\sum_{x_{g}} 1 /\left(16 \pi^{2} R_{s} R_{g}\right)\right)^{2}}{E\left[\left(\sum_{x_{g}} n\left(t=t_{0}\right)\right)^{2}\right]}=\frac{\left(\sum_{x_{g}} 1 /\left(16 \pi^{2} R_{s} R_{g}\right)\right)^{2}}{N_{x} \sigma^{2}} \\
& S_{N R_{\text {MIMO }}}=\frac{\left(\sum_{x_{s}, x_{g}} 1 /\left(16 \pi^{2} R_{s} R_{g}\right)\right)^{2}}{E\left[\left(\sum_{x_{s}, x_{g}} n\left(t=t_{0}\right)\right)^{2}\right]}=\frac{\left(\sum_{x_{s}, x_{g}} 1 /\left(16 \pi^{2} R_{s} R_{g}\right)\right)^{2}}{N_{x}^{2} \sigma^{2}}
\end{aligned}
$$

By using the corresponding array parameters as described in Subsection 3.2.1, one can calculate that the SNR of MIMO configuration is always greater than SISO and SIMO configurations over the imaging domain.

\subsubsection{Analysis on Resolving Capability over Dipping Surface}

Based on the Snell's law, a reflection on target surface can occur if the incident and reflection angles of the wave are equal. Suppose that $\hat{k}_{s}$ denotes a unit vector in direction of incident 
wave from the transmitter and $\hat{k}_{g}$ denotes a unit vector in direction of reflected wave to the receiver. Then, the Snell's law implies that the resultant $\hat{k}_{g}-\hat{k}_{s}$ must be in line with the normal vector of the surface $\mathrm{n}$ as formulated in (3.87).

$$
\hat{n}=\frac{\hat{k}_{g}-\hat{k}_{s}}{\left|\hat{k}_{g}-\hat{k}_{s}\right|}
$$

Since we have limited number of transmitters and receivers, at a particular point there will be only a limited number of $\hat{n}$ that can be resolved by the array. We can observe such properties by plotting all the possible $\hat{n}$ on several point in the imaging domain as shown in Fig. 3.26 for each array. As we can see, the MIMO configuration provides higher number of $\hat{n}$ compared to SISO and SIMO arrays, which implies better capability in resolving the dipping surface of the target.

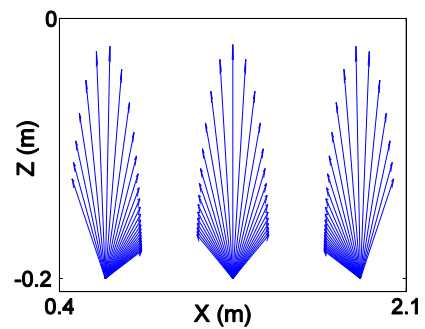

(a) SISO

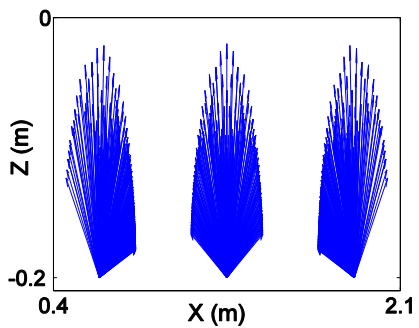

(c) MIMO-half

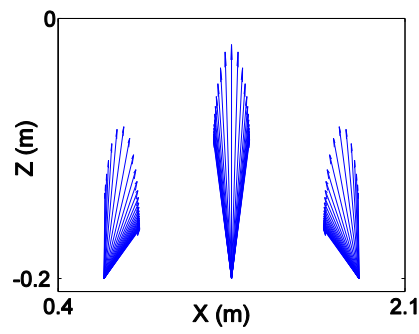

(b) SIMO

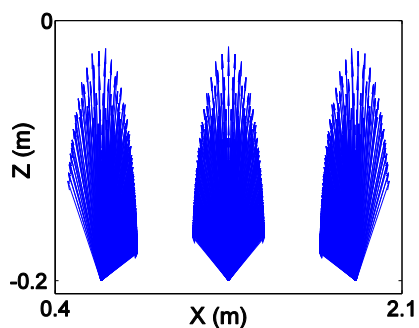

(d) MIMO-all

Fig. 3.26: Normal vectors that can be resolved by each array.

\subsection{Comparison of Migration Methods}

In Section 3.1, we have addressed 3 most common migration algorithms for GPR signal processing, namely Kirchhoff migration, phase-shift migration, and f-k migration. As described, these algorithms can handle both SISO and MIMO configurations. In [105], these migration algorithms were evaluated on SISO configuration and it was shown that each method yields different imaging performance. Meanwhile, in previous section we have 
demonstrated the imaging capabilities of SISO, SIMO, and MIMO arrays by using f-k migration. Since the migration algorithms also affect the imaging result, we extend our study herein over these migration algorithms to verify the improvement provided by MIMO configuration. In order to simplify the analysis, we will only repeat the evaluation for SISO and MIMO-all configurations. The latter one will be simply referred as MIMO configuration.

\subsubsection{Analysis on Beam-Pattern}

Similar to the previous study, we begin with analysis on PSF. We revisit the simulation on point target presented in Fig. 3.11. By processing the SISO and MIMO-all data with Kirchhoff migration, phase-shift migration, and $\mathrm{f}-\mathrm{k}$ migration, we obtain the PSFs of the corresponding algorithms.

There are 2 ways to extract beam-pattern in UWB imaging. The first method is by taking summation over depth dimension of the PSF [105], which is suitable to observe the side-lobe level of the PSF. The results are given in Fig. 3.27. Despite each migration method exhibits different side-lobe level, we can see that MIMO configuration still outperforms SISO configuration by providing lower side-lobe for all migration algorithms. Among all these algorithms, Kirchhoff migration has the highest side-lobes level. Since the simulation is performed in homogeneous medium, phase-shift migration and $\mathrm{f}-\mathrm{k}$ migration produce almost identical results. 


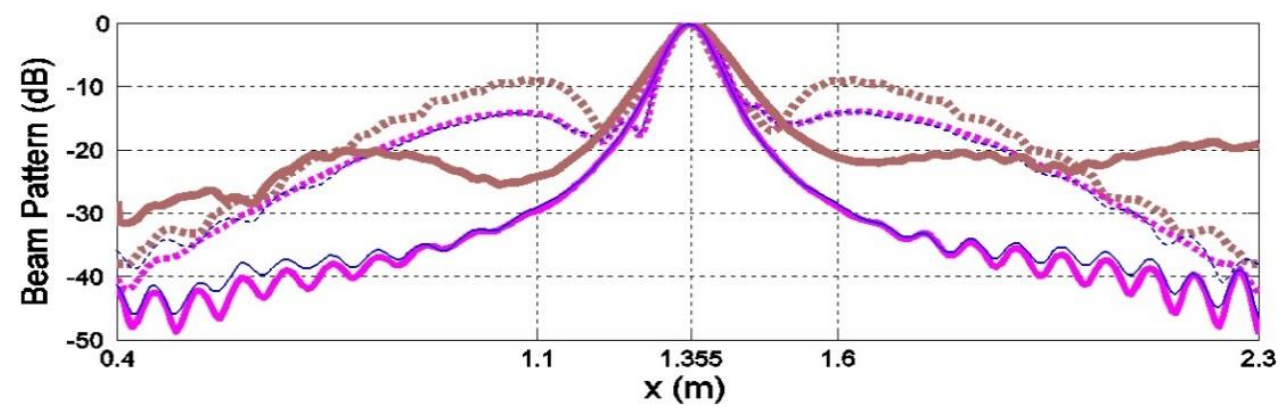

(a) Beam-patterns

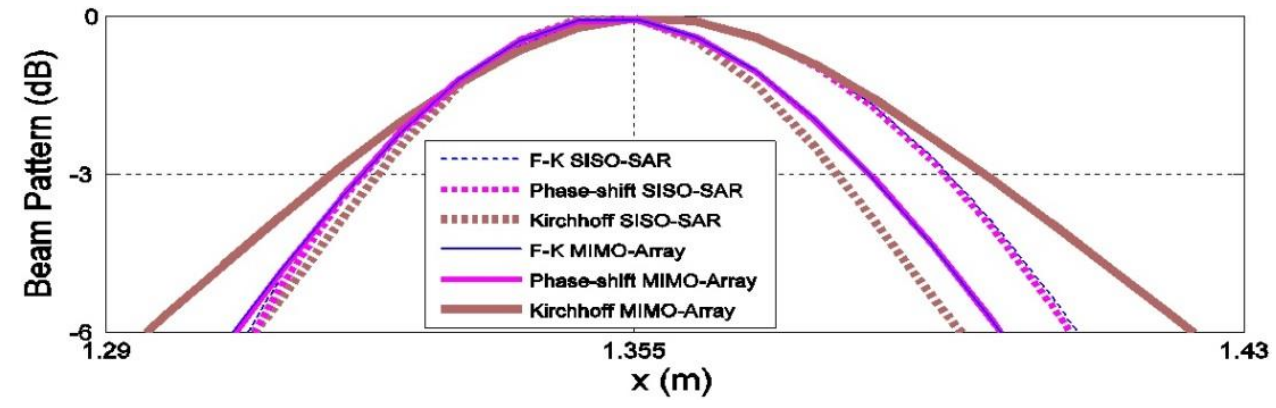

(b) Zoom-in of (a)

Fig. 3.27: Beam-patterns by using summation over depth dimension of the PSF.

The second method is by taking horizontal slice at the peak value of the PSF [106], which is more suitable for analyzing the resolution. The results are given in Fig. 3.28. These results clearly show that the resolution is also affected by migration algorithm. Among all these algorithms, Kirchhoff migration yields the best resolution. Similar to the side-lobe case, phase-shift migration and f-k migration produce identical resolutions. Finally, by comparing the 3-dB width of the beam-pattern of MIMO and SISO on each algorithm independently, we can observe that MIMO array still improves the resolution compared to SISO array for the same migration method. Kirchhoff migration on SISO array produces higher resolution than phase-shift migration and f-k migration on MIMO array, but at the expense of higher sidelobe level and computational complexity. 


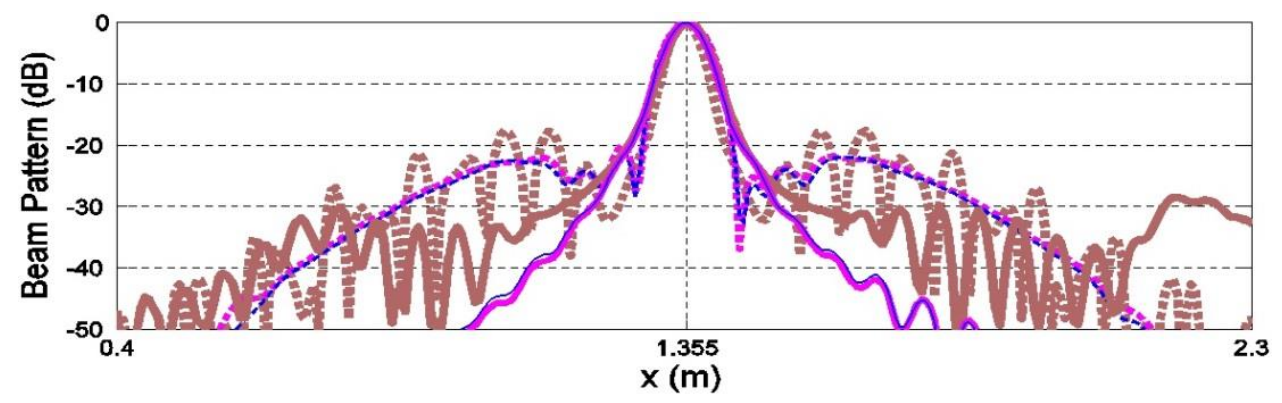

(a) Beam-patterns

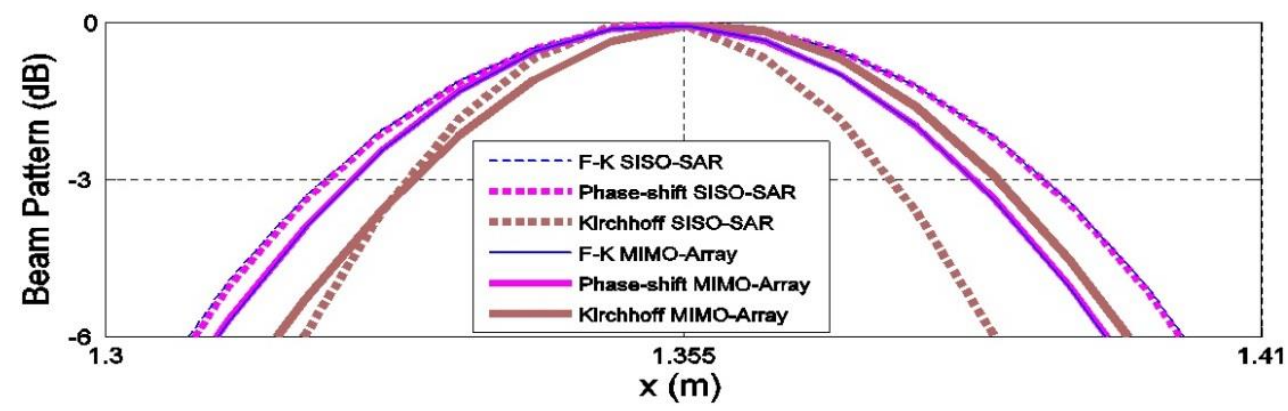

(b) Zoom-in of (a)

Fig. 3.28: Beam-patterns by taking horizontal slice at the peak of the PSF.

\subsubsection{Analysis on Distributed Targets}

Following the previous result, we perform another simulation that represents a more realistic case. The simulation contains 3 pairs of closely located pipes with diameter $3 \mathrm{~cm}$ as shown in Fig. 3.29. The distances between each pipe in the same pair are $30 \mathrm{~cm}, 11 \mathrm{~cm}$, and $20 \mathrm{~cm}$ from the left to the right respectively. The pipes are made of PEC material, while the subsurface is a lossless homogeneous concrete with relative permittivity of 6 and relative permeability of 1 .

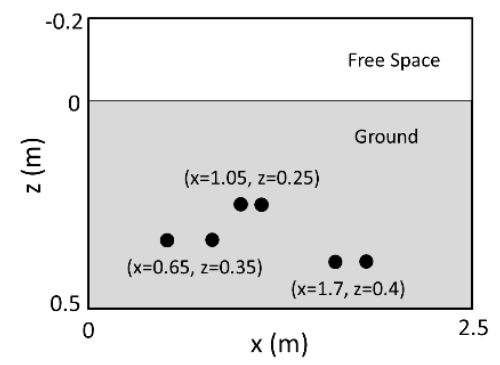

Fig. 3.29: Simulation configuration for analysis on distributed targets.

First, we process the data with Kirchhoff migration. The results are presented in Fig. 3.30. As we can see, Kirchhoff migration can resolve all the pipes successfully in both SISO and MIMO configurations, which indicates its ability in producing high resolution image. 
However, the migration results also suffer from high side-lobe level and several image artifacts.

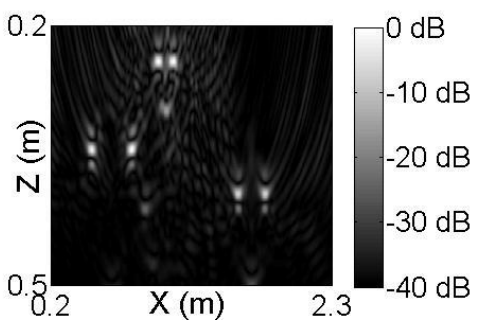

(a) SISO

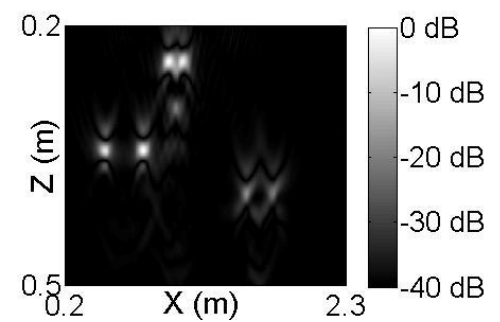

(b) MIMO

Fig. 3.30: Results of Kirchhoff migration.

Next, we apply phase-shift migration and f-k migration to the data. The results of phase-shift migration are given in Fig. 3.31, while the results of f-k migration are shown in Fig. 3.32. We can observe that these two algorithms yield similar results since the simulation is performed on homogeneous subsurface medium. Furthermore, by comparing with Fig. 3.30, one can see that phase-shift migration and f-k migration produce less side-lobe than Kirchhoff migration.

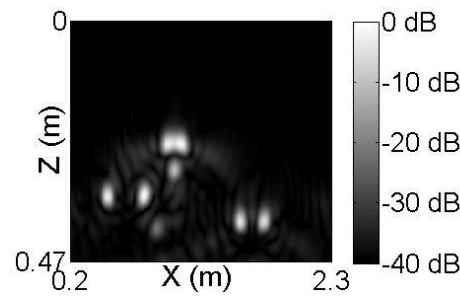

(a) SISO

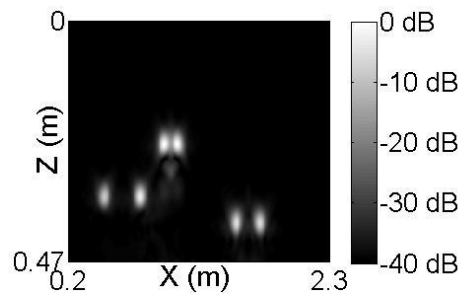

(b) MIMO

Fig. 3.31: Results of phase-shift migration.

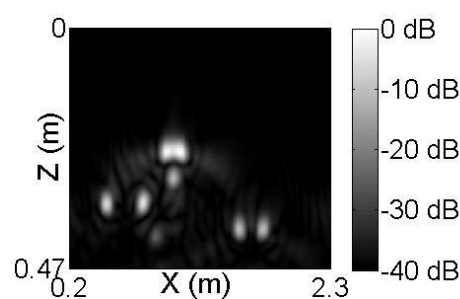

(a) SISO

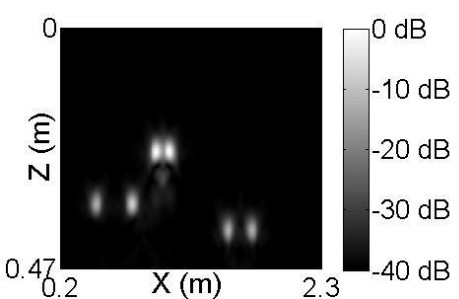

(b) MIMO

Fig. 3.32: Results of f-k migration.

If we compare the images produced by SISO and MIMO in Fig. 3.31 and Fig. 3.32, we can observe that the two pipes on the top, which have the smallest distance between each other, are seems to be overlapped in SISO images. Meanwhile, in MIMO images, such pipes are successfully resolved, which indicates a better resolution. Moreover, SISO array exhibits noisier image than MIMO array due to side-lobe and image artifacts. These results confirm 
the advantages provided by MIMO configuration in terms of resolution, side-lobe, and image artifacts.

Finally, we can summarize the performance of each migration algorithm on SISO and MIMO configurations as in Table 3.2.

Table 3.2: Summary of PeRformance OF EACH Migration AlgORITHM

\begin{tabular}{|l|c|c|c|}
\hline \multicolumn{1}{|c|}{ Parameter } & Kirchhoff migration & Phase-shift migration & F-k migration \\
\hline Resolution & Highest & Same as f-k migration & $\begin{array}{c}\text { Same as phase-shift } \\
\text { migration }\end{array}$ \\
\hline Side-lobe level & Highest & Same as f-k migration & $\begin{array}{c}\text { Same as phase-shift } \\
\text { migration }\end{array}$ \\
\hline $\begin{array}{l}\text { Computational } \\
\text { complexity }\end{array}$ & Highest & In-between & Lowest \\
\hline $\begin{array}{l}\text { Flexibility on sensor } \\
\text { configuration }\end{array}$ & Any configuration & Limited configuration & Limited configuration \\
\hline $\begin{array}{l}\text { Ability to handle } \\
\text { variation in medium }\end{array}$ & $\begin{array}{c}\text { Any inhomogeneous } \\
\text { medium }\end{array}$ & $\begin{array}{c}\text { Only vertically varying } \\
\text { medium }\end{array}$ & $\begin{array}{c}\text { Only homogeneous } \\
\text { medium }\end{array}$ \\
\hline
\end{tabular}

\subsection{Autofocusing Method}

In Subsection 3.1.3, we have introduced 2 typical approaches for velocity estimation in GPR signal processing. The first method extracts the velocity values by using additional measurement data in CMP gather, while the second method optimizes the measurement data directly with particular focusing parameter. The latter method, which is well-known as autofocusing, has been used with a number of variations on SISO configuration [99]-[102]. We can also apply such method directly on MIMO configuration. However, due to the large data size in MIMO configuration, the computational time becomes extremely high. To resolve these shortcomings, in this section we present an autofocusing technique that can handle MIMO data efficiently. This method combines the original autofocusing method with particular migration to zero-offset (MZO) operator that can transform MIMO data into equivalent SISO data.

\subsubsection{MZO Operator}

In seismic imaging, MZO is an operator that transforms a set of data collected with separated transmitter and receiver into equivalent zero-offset measurement data. This operator can be performed in time and spatial domain [96] or in frequency and wavenumber domain [107], which uses Fourier transform-based equation. In the proposed method, we use MZO operator developed by Hale in [107], in which MZO is carried out in frequency and wavenumber 
domain. The rationale in choosing this operator is to combine such technique with f-k migration algorithm, which also works in frequency and wavenumber domain. We will describe this MZO operator in the following.

We recall the f-k migration steps on MIMO configuration as given in Fig. 3.33.

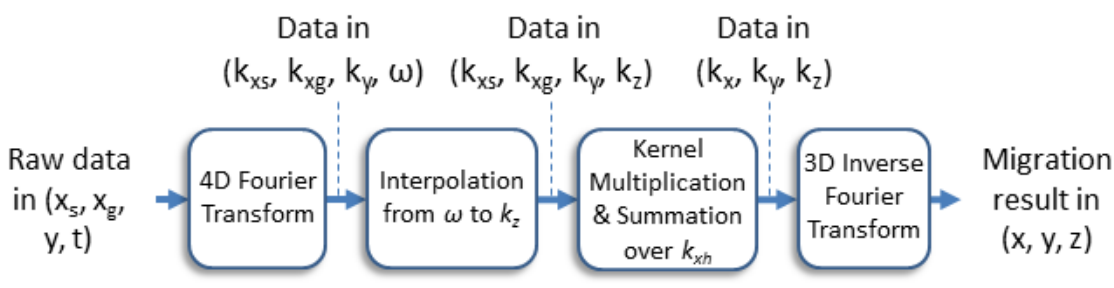

Fig. 3.33: F-k migration steps for MIMO configuration.

In the interpolation part, the data is transformed from $\omega$ into $k_{z}$, where $k_{z}$ is given in (3.88) for $2 \mathrm{D}$ case and (3.89) for 3D case.

$$
\begin{gathered}
k_{z(2 D)}=-\frac{\omega}{c}\left(\sqrt{1-\left(\frac{c k_{x g}}{\omega}\right)^{2}}+\sqrt{1-\left(\frac{c k_{x s}}{\omega}\right)^{2}}\right) \\
k_{z(3 D)}=k_{z(2 D)} \sqrt{1-\left(\frac{k_{y}}{k_{z(2 D)}}\right)^{2}}
\end{gathered}
$$

Hale [107] decomposed this step into a cascade of 2 interpolation processes. The first part is the interpolation from frequency variable $\omega$ into intermediate frequency variable $\omega_{0}$, which is well-known as MZO interpolation. This step can be expressed as (3.90) for 2D case and (3.91) for 3D case.

$$
\begin{aligned}
& \omega=\omega_{0} \sqrt{1+\left(\frac{k_{x h}}{k_{z(2 D)}}\right)^{2}} \\
& \omega=\omega_{0} \sqrt{1+\left(\frac{k_{x h}}{k_{z(3 D)}}\right)^{2}}
\end{aligned}
$$

The second part is the interpolation from $\omega_{0}$ to $k_{z}$, which is equivalent to the interpolation step in SISO f-k migration. This part can be formulated as (3.92) for 2D case and (3.93) for 3D case. 


$$
\begin{gathered}
k_{z(2 D)}=-\frac{2 \omega_{0}}{c} \sqrt{1-\left(\frac{c k_{x}}{2 \omega_{0}}\right)^{2}} \\
k_{z(3 D)}=-\frac{2 \omega_{0}}{c} \sqrt{1-\left(\frac{c k_{x}}{2 \omega_{0}}\right)^{2}-\left(\frac{c k_{y}}{2 \omega_{0}}\right)^{2}}
\end{gathered}
$$

Since the offset wavenumber $k_{x h}$ only appears in the first part, we can eliminate this variable afterward by performing kernel multiplication and summation over $k_{x h}$ before proceeding to the second part. This step is given by (3.94) for 2D case and (3.95) for 3D case. Such process will produce data that depends only on $k_{x}, k_{y}$, and $\omega_{0}$, which implies a transformation of MIMO data into its equivalent SISO data. Then, we can apply the original autofocusing method on this SISO data.

$$
\begin{gathered}
u_{M Z O}\left(k_{x}, \omega_{0}\right)=\int\left|\frac{k_{z g} k_{z s} \omega_{0}}{\pi k_{z}^{2} \omega}\right| u\left(k_{x}, k_{x h}, \omega\right) d k_{x h} \\
u_{M Z O}\left(k_{x}, k_{y}, \omega_{0}\right)=\int\left|\frac{k_{z g} k_{z s} \omega_{0}}{\pi k_{z}^{2} \omega}\right| u\left(k_{x}, k_{y}, k_{x h}, \omega\right) d k_{x h}
\end{gathered}
$$

\subsubsection{Proposed Autofocusing Method}

In the previous subsection, we have introduced the $\mathrm{MZO}$ operator that can reduce the size of MIMO data into an equivalent SISO data. Therefore, the next step is to embed this operator into the autofocusing method for SISO configuration.

There are various focusing parameter that have been proposed in the existing autofocusing methods. To decide which parameter to use, we first observe the effect of incorrect wave velocity in the migration image. There are 2 cases that can occur when the image is migrated with wrong velocity. The first case is under-migrated, which occurs when the velocity is too low. A point target in under-migrated condition will appear as diffraction frown as shown in Fig. 3.34(a). The second case is over-migrated, which occurs when the velocity is too high. For point target, over-migrated will produce a diffraction smile as given in Fig. 3.34(b). On the other hand, a correct wave velocity will generate a focused image as shown in Fig. 3.34(c) for point target case. 


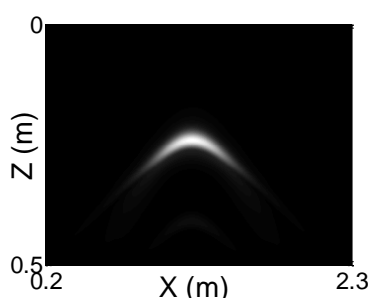

(a) Under-migrated

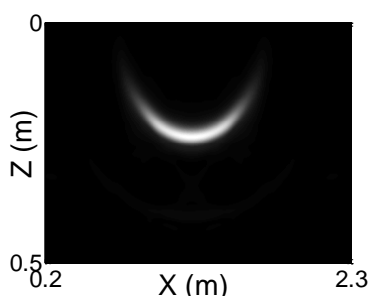

(b) Over-migrated

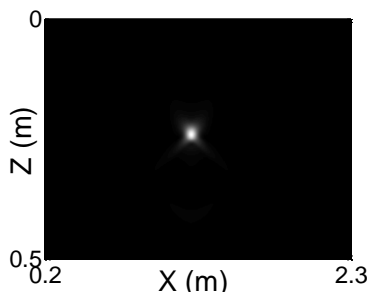

(c) Correct velocity

Fig. 3.34: Migration on a point target with different wave velocities.

The presented example on point target case demonstrates the effect of wave velocity in which the target reflection becomes more concentrated as the migration velocity approaches the correct velocity. This result implies that the correct velocity will produce image with smaller entropy than the under-migrated and over-migrated cases. Therefore, it is reasonable to use entropy of the image as the parameter of correct migration velocity.

In our autofocusing method, we use inverse varimax $\left(V^{-1}\right)[101]$ as the focusing indicator as given in (3.96), where $x(m, n)$ denotes the pixel of the image. This parameter is equivalent with entropy but has better stability in the calculation.

$$
V^{-1}=\frac{\left(\sum_{m, n} x^{2}(m, n)\right)^{2}}{\sum_{m, n} x^{4}(m, n)}
$$

Finally, by combining MZO operator with $\mathrm{f}-\mathrm{k}$ migration and inverse varimax, we can formulate the autofocusing method for MIMO configuration as shown in Fig. 3.35. First, the MIMO data is converted into frequency wavenumber domain. Then, we apply the MZO operator to transform this MIMO data into its equivalent SISO data. Next, optimization is carried out by performing SISO f-k migration on several values of wave velocity. The optimum velocity is taken as the velocity value that minimizes the inverse varimax of the image. 


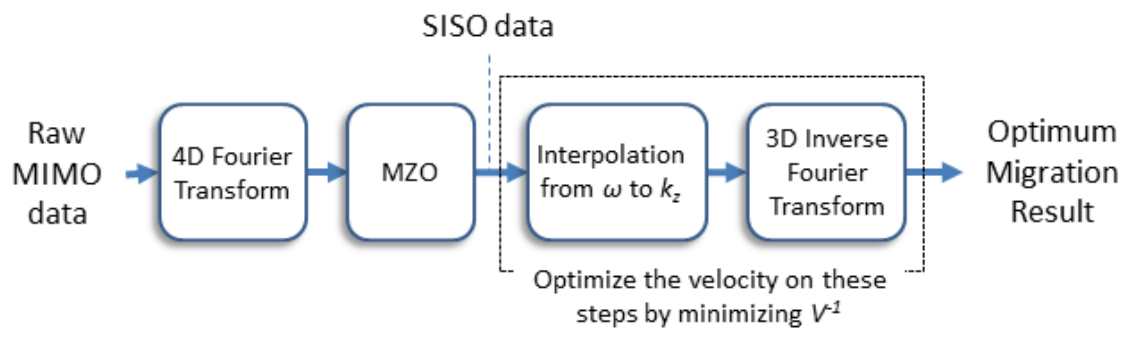

Fig. 3.35: The proposed autofocusing algorithm.

\subsubsection{Results and Performance Analysis}

In this subsection, we test the proposed autofocusing algorithm based on simulation data. In order to compare its performance, we also perform autofocusing on SISO data and MIMO data directly without MZO operator. The latter case will be referred as standard MIMO autofocusing. The standard autofocusing on SISO is illustrated in Fig. 3.36, while the standard autofocusing on MIMO is given in Fig. 3.37.

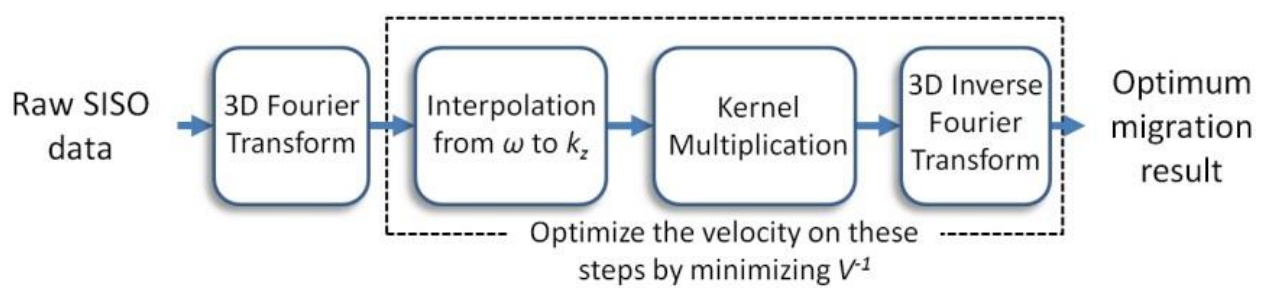

Fig. 3.36: Standard autofocusing on SISO configuration.

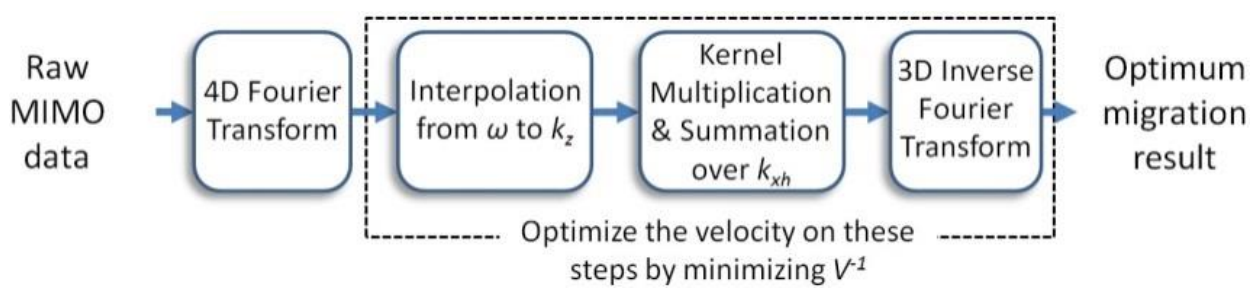

Fig. 3.37: Standard autofocusing on MIMO configuration.

We consider 3 simulation scenarios with different target shapes as shown in Fig. 3.38 to Fig. 3.40. The first simulation scheme contains 3 pairs of closely located pipes with diameter of 3 $\mathrm{cm}$ and offset of $11 \mathrm{~cm}$. The pipes are made of perfect electric conductor material, while the subsurface is homogeneous with relative permittivity 6 , relative permeability 1 , and conductivity $0.001 \mathrm{~S} / \mathrm{m}$. This scenario is related to utilities mapping application. 


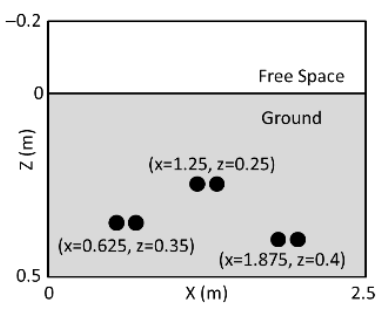

(a) $2 \mathrm{D}$ case

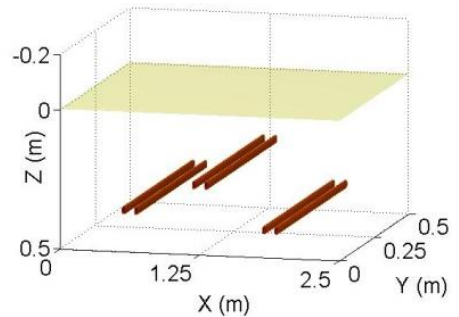

(b) $3 \mathrm{D}$ case

Fig. 3.38: Target configuration in simulation 1 .

In the second simulation, the targets are 3 rectangular voids with sizes $5 \times 5 \times 5 \mathrm{~cm}, 10 \times 10 \times 10$ $\mathrm{cm}$, and $20 \times 20 \times 20 \mathrm{~cm}$ respectively from the left to the right. This scheme represents the void detection case in inspection of infrastructure such as building, road, pavement, bridge, and etc. Similar to the previous scheme, the subsurface has relative permittivity of 6 , relative permeability of 1 , and conductivity of $0.001 \mathrm{~S} / \mathrm{m}$

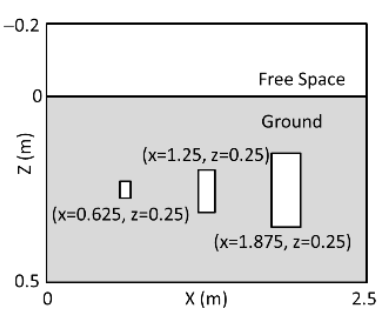

(a) $2 \mathrm{D}$ case

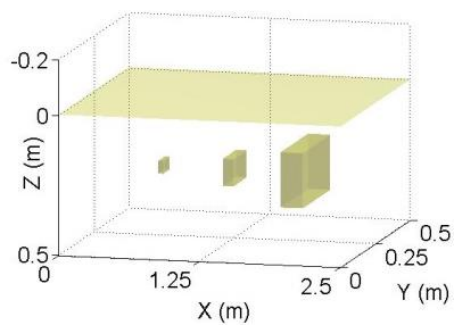

(b) $3 \mathrm{D}$ case

Fig. 3.39: Target configuration in simulation 2 .

Finally, the last simulation is related to landmines detection in military application. Several landmine targets are placed at different locations as shown in Fig. 3.40. In the 3D case, the landmines are located at $y=0.15 \mathrm{~m}, y=0.25 \mathrm{~m}$, and $y=0.35 \mathrm{~m}$ from the most left to the right respectively. We use landmine model proposed in [108] as given in Fig. 3.41. We also add AWGN noise to the third simulation with equal power on each A-scan sample. In this simulation, the subsurface is homogeneous with relative permittivity 5 , relative permeability 1 , and conductivity $0.0001 \mathrm{~S} / \mathrm{m}$.

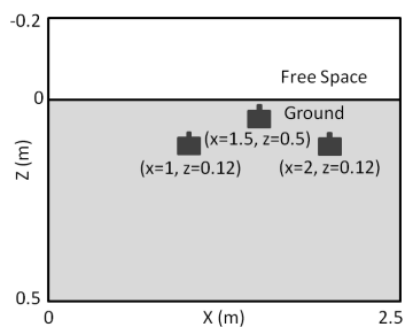

(a) $2 \mathrm{D}$ case

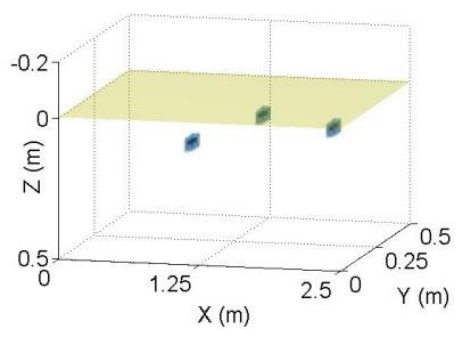

(b) $3 \mathrm{D}$ case

Fig. 3.40: Target configuration in simulation 3. 


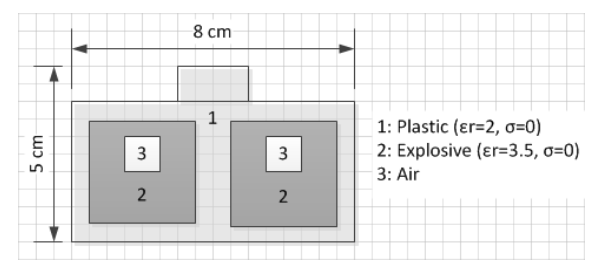

Fig. 3.41: Landmine model for simulation 3.

We carry out the simulation in both 2D and 3D environments, and then apply the standard SISO autofocusing, the standard MIMO autofocusing, and the proposed MIMO autofocusing methods on the resulting data. Since we have low loss subsurface, the conductivity part can be omitted so that the optimization on wave velocity is simplified into the optimization on relative permittivity.

Fig. 3.42 presents the result of the first simulation for 2D case. As can be clearly seen, all the pipes are successfully focused by the three autofocusing algorithms. The resulting optimum relative permittivities are 4.375 for the standard SISO autofocusing, 4.0 for the standard MIMO autofocusing, and 4.375 for the proposed MIMO autofocusing. Furthermore, the SISO image in Fig. 3.42(a) exhibits noisier result and unclear separation between the pipes in the same pair, while both MIMO autofocusing results in Fig. 3.42(b) and (c) can resolve the two closely located pipes and provide cleaner images. These results indicate that the benefits of MIMO configuration in terms of higher resolution and lower side-lobe are preserved by the proposed autofocusing method. Meanwhile, the drawback of this method can be seen in Fig. 3.42(c), where some image artifacts arise between the two closely located pipes. These artifacts are caused by the incorrect initial wave velocity in the MZO process. However, these artifacts will be reduced if the corresponding initial value is not too far from the correct velocity value. 


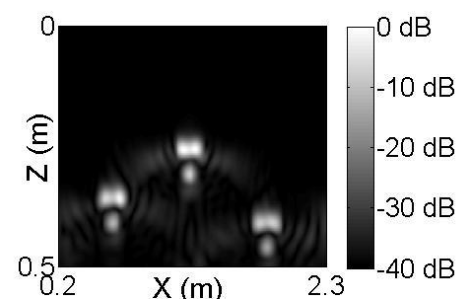

(a) Standard SISO autofocusing

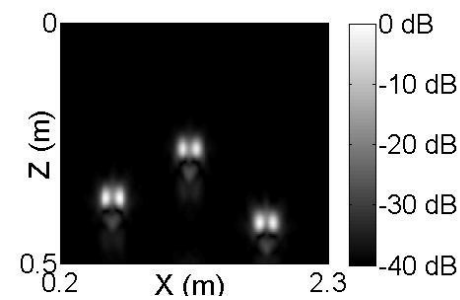

(b) Standard MIMO autofocusing

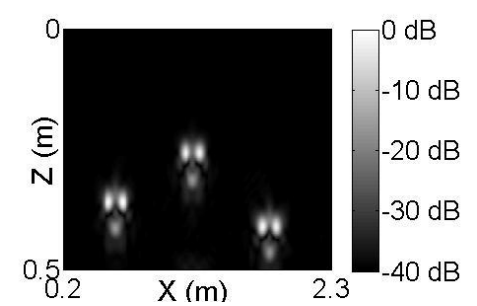

(c) Proposed MIMO autofocusing

Fig. 3.42: Results of simulation 1 for 2D case.

The results of simulation 1 for 3D case are given in Fig. 3.43 with $-5 \mathrm{~dB}$ dynamic range on the normalized data. We can see that these results are in agreement with the $2 \mathrm{D}$ case. The optimum relative permittivites for 3D case are 4.75 for the standard SISO autofocusing, 3.625 for the standard MIMO autofocusing, and 4.75 for the proposed MIMO autofocusing.

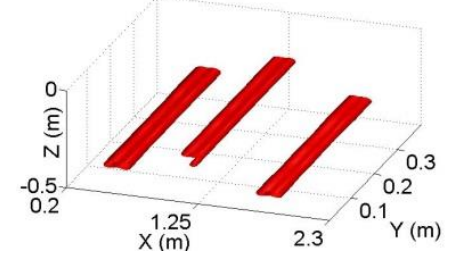

(a) Standard SISO autofocusing

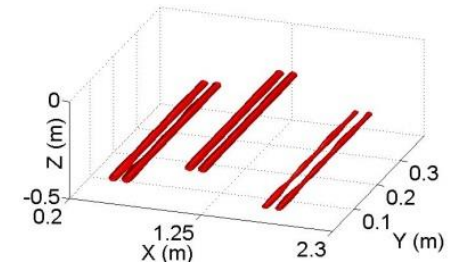

(b) Standard MIMO autofocusing

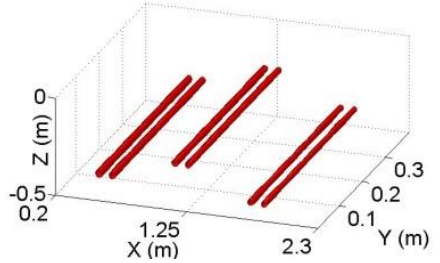

(c) Proposed MIMO autofocusing

Fig. 3.43: Results of simulation 1 for 3D case.

The results of the second simulation are presented in Fig. 3.44 for 2D case and Fig. 3.45 for 3D case. Similar to the results of simulation 1 , all the 3 voids are successfully focused. In the 2D case, the optimum relative permittivities are $4.375,3.25$, and 4.375 for the standard SISO autofocusing, the standard MIMO autofocusing, and the proposed MIMO autofocusing respectively. Meanwhile, the optimum relative permittivities in 3D case are 8.5, 4.375, and 5.875 for the standard SISO autofocusing, the standard MIMO autofocusing, and the 
proposed MIMO autofocusing respectively. Notice that these optimum permittivity values are different from that of simulation 1, indicating the dependence of autofocusing results on the shapes of the targets.

In Fig. 3.45(b) and (c), we can observe that the reflection of the smallest void for both MIMO images has higher amplitude (up to $4 \mathrm{~dB}$ ) that the result of SISO image in Fig. 3.45(a) (-15 $\mathrm{dB})$. This result implies that the capability of MIMO array in detecting small target is also preserved by the proposed autofocusing method.

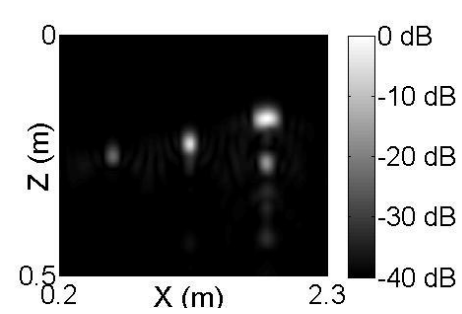

(a) Standard SISO autofocusing

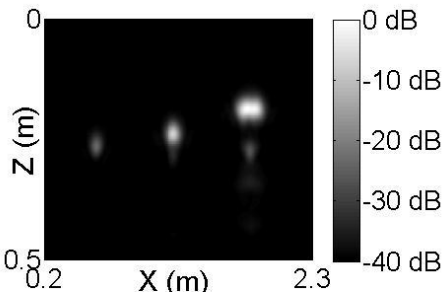

(b) Standard MIMO autofocusing

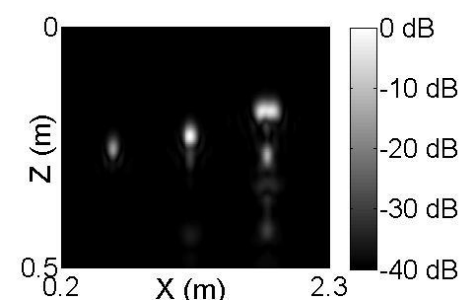

(c) Proposed MIMO autofocusing

Fig. 3.44: Results of simulation 2 for $2 \mathrm{D}$ case.

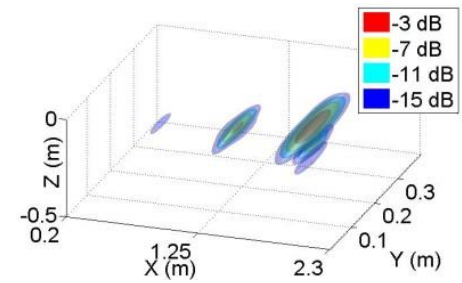

(a) Standard SISO autofocusing

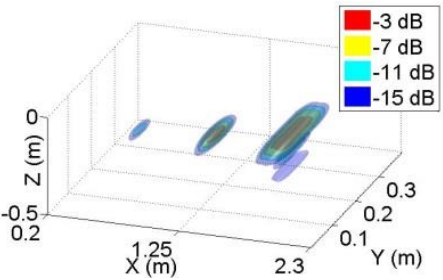

(b) Standard MIMO autofocusing

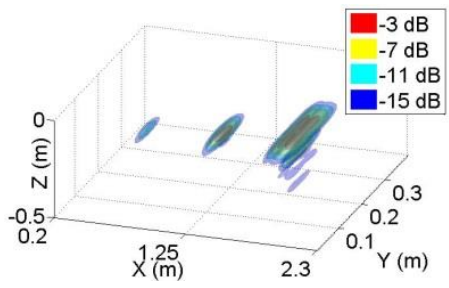

(c) Proposed MIMO autofocusing

Fig. 3.45: Results of simulation 2 for 3D case.

The results of the third simulation are shown in Fig. 3.46 for 2D case and Fig. 3.47 for 3D case. This simulation demonstrates the autofocusing performance when the noise components are taken into account. As we can see, the landmine targets are still well-focused by all these 
autofocusing techniques. The optimum relative permittivities for $2 \mathrm{D}$ case are $2.875,2.875$, and 2.875 for the standard SISO autofocusing, the standard MIMO autofocusing, and the proposed MIMO autofocusing respectively. Meanwhile, the optimum relative permittivities for $3 \mathrm{D}$ case are $3.625,3.25$, and 3.25 for the standard SISO autofocusing, the standard MIMO autofocusing, and the proposed MIMO autofocusing respectively. In addition, we can observe that under the same noise power, both MIMO autofocusing methods yield cleaner images compared to SISO autofocusing. These results indicate that the advantages of MIMO array in providing higher SNR is successfully preserved by the autofocusing methods.

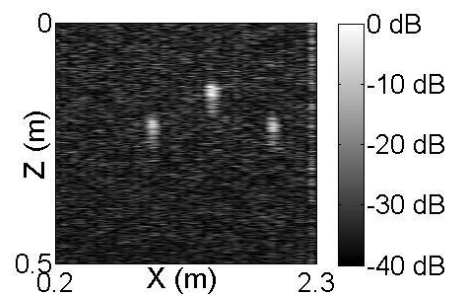

(a) Standard SISO autofocusing

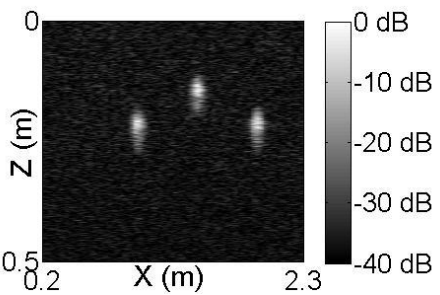

(b) Standard MIMO autofocusing

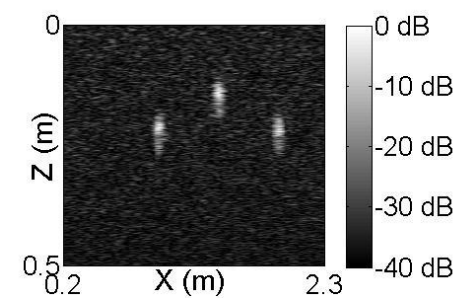

(c) Proposed MIMO autofocusing

Fig. 3.46: Results of simulation 3 for 2D case.

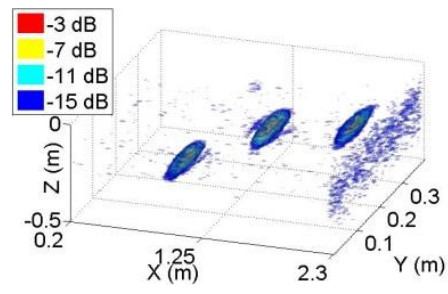

(a) Standard SISO autofocusing

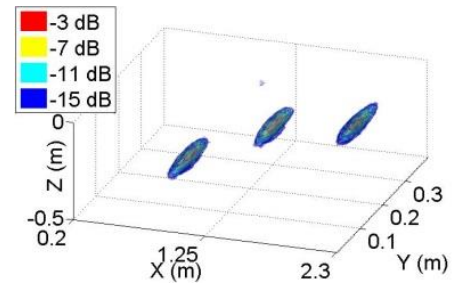

(b) Standard MIMO autofocusing

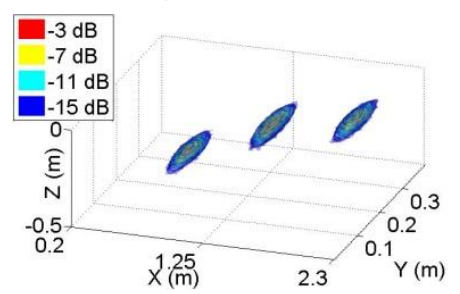

(c) Proposed MIMO autofocusing

Fig. 3.47: Results of simulation 3 for 3D case. 
The average computation time of the 3 autofocusing techniques are given in Table 3.3. The results clearly show that the proposed autofocusing method reduces the computation time significantly compared to the direct autofocusing method on MIMO data.

Table 3.3: Average Computation Time of the Autofocusing Methods

\begin{tabular}{|c|c|c|c|c|c|c|}
\hline \multirow{2}{*}{ Autofocusing Method } & \multicolumn{4}{|c|}{ Average Time (s) } \\
\cline { 2 - 7 } & \multicolumn{2}{|c|}{ Simulation 1 } & \multicolumn{2}{c|}{ Simulation 2 } & \multicolumn{2}{c|}{ Simulation 3 } \\
\cline { 2 - 7 } & 2D & 3D & 2D & 3D & 2D & 3D \\
\hline SISO & 2.68 & 68.44 & 2.82 & 72.40 & 2.45 & 69.03 \\
\hline MIMO & 34.52 & $1.29 \times 10^{3}$ & 34.12 & $1.29 \times 10^{3}$ & 30.54 & $1.03 \times 10^{3}$ \\
\hline MIMO with MZO & 5.33 & 147.08 & 5.32 & 146.74 & 4.95 & 142.98 \\
\hline
\end{tabular}

To support these results numerically, we calculate in the following the computational complexity of each algorithm. Let $N_{x}$ denotes the number of transmitter and receiver, $N_{t}$ denotes the number of time sample, $N_{y}$ denotes the number of sample on $y$-axis, $N_{z}$ denotes the number of sample along $z$-axis, $N_{e}$ is the number of velocity values employed in the optimization. We assume that 1-D $N$-samples FFT and IFFT algorithms have complexity of $3 N \log _{2} N$, and the complexity of an interpolation process is $M_{\text {interp }}$. A complex addition involves 2 real operations, while a complex multiplication involves 6 real operations. We also assume that a real addition and a real multiplication have the same cost.

The computational complexity for 2D and 3D data on the standard SISO autofocusing, the standard MIMO autofocusing, and the proposed MIMO autofocusing are listed in Tables 3.4 to 3.6 respectively. We can observe that the FFT and IFFT steps on each autofocusing method give almost similar complexity. Meanwhile, the interpolation step, kernel multiplication step, and summation over $k_{x h}$ produce significant differences on the complexity of each algorithm. In general, the MZO operator transforms MIMO data over these steps, whose dimension is $N_{x}^{2} \times N_{y} \times N_{t}$ for 3D case and $N_{x}^{2} \times N_{t}$ for 2D case, into SISO data, whose dimension is $\left(2 N_{x}-1\right) \times N_{y} \times N_{t}$ for $3 \mathrm{D}$ case and $\left(2 N_{x}-1\right) \times N_{t}$ for $2 \mathrm{D}$ case. Therefore, the computational burden during the optimization process is reduced, which in turns accelerates the autofocusing process.

Table 3.4: COMPUTATIONAL COMPLEXITY OF STANDARD SISO AUTOFOCUSING

\begin{tabular}{|l|c|c|}
\hline \multicolumn{1}{|c|}{ Steps } & Complexity on 2D Data & Complexity on 3D Data \\
\hline FFT over $\mathrm{x}$ & $3 \mathrm{~N}_{\mathrm{t}} \mathrm{N}_{\mathrm{x}} \log _{2}\left(\mathrm{~N}_{\mathrm{x}}\right)$ & $3 \mathrm{~N}_{\mathrm{t}} \mathrm{N}_{\mathrm{x}} \mathrm{N}_{\mathrm{y}} \log _{2}\left(\mathrm{~N}_{\mathrm{x}}\right)$ \\
\hline FFT over $\mathrm{y}$ & - & $3 \mathrm{~N}_{\mathrm{t}} \mathrm{N}_{\mathrm{x}} \mathrm{N}_{\mathrm{y}} \log _{2}\left(\mathrm{~N}_{\mathrm{y}}\right)$ \\
\hline
\end{tabular}




\begin{tabular}{|c|c|c|} 
FFT over $t$ & $3 \mathrm{~N}_{\mathrm{t}} \mathrm{N}_{\mathrm{x}} \log _{2}\left(\mathrm{~N}_{\mathrm{t}}\right)$ & $3 \mathrm{~N}_{\mathrm{t}} \mathrm{N}_{\mathrm{x}} \mathrm{N}_{\mathrm{y}} \log _{2}\left(\mathrm{~N}_{\mathrm{t}}\right)$ \\
\hline Iterate over each velocity values: & & \\
\hline Interpolation $\omega$ to $\mathrm{k}_{\mathrm{z}}$ & $\mathrm{M}_{\mathrm{interp}} \mathrm{N}_{\mathrm{x}} \mathrm{N}_{\mathrm{z}} \mathrm{N}_{\mathrm{e}}$ & $\mathrm{M}_{\text {interp }} \mathrm{N}_{\mathrm{x}} \mathrm{N}_{\mathrm{y}} \mathrm{N}_{\mathrm{z}} \mathrm{N}_{\mathrm{e}}$ \\
\hline Kernel multiplication & $6 \mathrm{~N}_{\mathrm{x}} \mathrm{N}_{\mathrm{z}} \mathrm{N}_{\mathrm{e}}$ & $6 \mathrm{~N}_{\mathrm{x}} \mathrm{N}_{\mathrm{y}} \mathrm{N}_{\mathrm{z}} \mathrm{N}_{\mathrm{e}}$ \\
\hline IFFT over $\mathrm{k}_{\mathrm{x}}$ & $3 \mathrm{~N}_{\mathrm{x}} \mathrm{N}_{\mathrm{z}} \mathrm{N}_{\mathrm{e}} \log _{2}\left(\mathrm{~N}_{\mathrm{x}}\right)$ & $3 \mathrm{~N}_{\mathrm{x}} \mathrm{N}_{\mathrm{y}} \mathrm{N}_{\mathrm{z}} \mathrm{N}_{\mathrm{e}} \log _{2}\left(\mathrm{~N}_{\mathrm{x}}\right)$ \\
\hline IFFT over $\mathrm{k}_{\mathrm{y}}$ & - & $3 \mathrm{~N}_{\mathrm{x}} \mathrm{N}_{\mathrm{y}} \mathrm{N}_{\mathrm{z}} \mathrm{N}_{\mathrm{e}} \log _{2}\left(\mathrm{~N}_{\mathrm{y}}\right)$ \\
\hline IFFT over $\mathrm{k}_{\mathrm{z}}$ & $3 \mathrm{~N}_{\mathrm{x}} \mathrm{N}_{\mathrm{z}} \mathrm{N}_{\mathrm{e}} \log _{2}\left(\mathrm{~N}_{\mathrm{z}}\right)$ & $3 \mathrm{~N}_{\mathrm{x}} \mathrm{N}_{\mathrm{y}} \mathrm{N}_{\mathrm{z}} \mathrm{N}_{\mathrm{e}} \log _{2}\left(\mathrm{~N}_{\mathrm{z}}\right)$ \\
\hline Varimax Calculation & $6 \mathrm{~N}_{\mathrm{x}} \mathrm{N}_{\mathrm{z}} \mathrm{N}_{\mathrm{e}}$ & $6 \mathrm{~N}_{\mathrm{x}} \mathrm{N}_{\mathrm{y}} \mathrm{N}_{\mathrm{z}} \mathrm{N}_{\mathrm{e}}$ \\
\hline
\end{tabular}

Table 3.5: COMPUTATIONAL COMPLEXITY OF STANDARD MIMO AUTOFOCUSING

\begin{tabular}{|l|c|c|}
\hline \multicolumn{1}{|c|}{ Steps } & Complexity on 2D Data & Complexity on 3D Data \\
\hline FFT over $\mathrm{x}_{\mathrm{s}}$ & $3 \mathrm{~N}_{\mathrm{t}} \mathrm{N}_{\mathrm{x}}{ }^{2} \log _{2}\left(\mathrm{~N}_{\mathrm{x}}\right)$ & $3 \mathrm{~N}_{\mathrm{t}} \mathrm{N}_{\mathrm{x}}{ }^{2} \mathrm{~N}_{\mathrm{y}} \log _{2}\left(\mathrm{~N}_{\mathrm{x}}\right)$ \\
\hline FFT over $\mathrm{x}_{\mathrm{g}}$ & $3 \mathrm{~N}_{\mathrm{t}} \mathrm{N}_{\mathrm{x}}{ }^{2} \log _{2}\left(\mathrm{~N}_{\mathrm{x}}\right)$ & $3 \mathrm{~N}_{\mathrm{t}} \mathrm{N}_{\mathrm{x}}{ }^{2} \mathrm{~N}_{\mathrm{y}} \log _{2}\left(\mathrm{~N}_{\mathrm{x}}\right)$ \\
\hline FFT over $\mathrm{y}$ & - & $3 \mathrm{~N}_{\mathrm{t}} \mathrm{N}_{\mathrm{x}}{ }^{2} \mathrm{~N}_{\mathrm{y}} \log _{2}\left(\mathrm{~N}_{\mathrm{y}}\right)$ \\
\hline FFT over $\mathrm{t}$ & $3 \mathrm{~N}_{\mathrm{t}} \mathrm{N}_{\mathrm{x}}{ }^{2} \log _{2}\left(\mathrm{~N}_{\mathrm{t}}\right)$ & $3 \mathrm{~N}_{\mathrm{t}} \mathrm{N}_{\mathrm{x}}{ }^{2} \mathrm{~N}_{\mathrm{y}} \log _{2}\left(\mathrm{~N}_{\mathrm{t}}\right)$ \\
\hline Iterate over each velocity values: & & \\
\hline Interpolation $\omega$ to $\mathrm{k}_{\mathrm{z}}$ & $\mathrm{M}_{\mathrm{interp}} \mathrm{N}_{\mathrm{x}}{ }^{2} \mathrm{~N}_{\mathrm{z}} \mathrm{N}_{\mathrm{e}}$ & $\mathrm{M}_{\mathrm{interp}} \mathrm{N}_{\mathrm{x}}{ }_{\mathrm{z}} \mathrm{N}_{\mathrm{y}} \mathrm{N}_{\mathrm{z}} \mathrm{N}_{\mathrm{e}}$ \\
\hline Kernel multiplication & $6 \mathrm{~N}_{\mathrm{x}}{ }^{2} \mathrm{~N}_{\mathrm{z}} \mathrm{N}_{\mathrm{e}}$ & $6 \mathrm{~N}_{\mathrm{x}}{ }^{2} \mathrm{~N}_{\mathrm{y}} \mathrm{N}_{\mathrm{z}} \mathrm{N}_{\mathrm{e}}$ \\
\hline Summation over $\mathrm{k}_{\mathrm{xh}}$ & $2\left(\mathrm{~N}_{\mathrm{x}}-1\right)^{2} \mathrm{~N}_{\mathrm{z}} \mathrm{N}_{\mathrm{e}}$ & $2\left(\mathrm{~N}_{\mathrm{x}}-1\right)^{2} \mathrm{~N}_{\mathrm{y}} \mathrm{N}_{\mathrm{z}} \mathrm{N}_{\mathrm{e}}$ \\
\hline IFFT over $\mathrm{k}_{\mathrm{x}}$ & $3\left(2 \mathrm{~N}_{\mathrm{x}}-1\right) \mathrm{N}_{\mathrm{z}} \mathrm{N}_{\mathrm{e}} \log _{2}\left(2 \mathrm{~N}_{\mathrm{x}}-1\right)$ & $3\left(2 \mathrm{~N}_{\mathrm{x}}-1\right) \mathrm{N}_{\mathrm{y}} \mathrm{N}_{\mathrm{z}} \mathrm{N}_{\mathrm{e}} \log _{2}\left(2 \mathrm{~N}_{\mathrm{x}}-1\right)$ \\
\hline IFFT over $\mathrm{k}_{\mathrm{y}}$ & - & $3\left(2 \mathrm{~N}_{\mathrm{x}}-1\right) \mathrm{N}_{\mathrm{y}} \mathrm{N}_{\mathrm{z}} \mathrm{N}_{\mathrm{e}} \log _{2}\left(\mathrm{~N}_{\mathrm{y}}\right)$ \\
\hline IFFT over $\mathrm{k}_{\mathrm{z}}$ & $3\left(2 \mathrm{~N}_{\mathrm{x}}-1\right) \mathrm{N}_{\mathrm{z}} \mathrm{N}_{\mathrm{e}} \log _{2}\left(\mathrm{~N}_{\mathrm{z}}\right)$ & $3\left(2 \mathrm{~N}_{\mathrm{x}}-1\right) \mathrm{N}_{\mathrm{y}} \mathrm{N}_{\mathrm{z}} \mathrm{N}_{\mathrm{e}} \log _{2}\left(\mathrm{~N}_{\mathrm{z}}\right)$ \\
\hline Varimax Calculation & $6\left(2 \mathrm{~N}_{\mathrm{x}}-1\right) \mathrm{N}_{\mathrm{z}} \mathrm{N}_{\mathrm{e}}$ & $6\left(2 \mathrm{~N}_{\mathrm{x}}-1\right) \mathrm{N}_{\mathrm{y}} \mathrm{N}_{\mathrm{z}} \mathrm{N}_{\mathrm{e}}$ \\
\hline
\end{tabular}

Table 3.6: COMPUTATIONAL COMPLEXITY OF THE PROPOSED MIMO AUTOFOCUSING

\begin{tabular}{|l|c|c|}
\hline \multicolumn{1}{|c|}{ Steps } & Complexity on 2D Data & Complexity on 3D Data \\
\hline FFT over $\mathrm{x}_{\mathrm{s}}$ & $3 \mathrm{~N}_{\mathrm{t}} \mathrm{N}_{\mathrm{x}}{ }^{2} \log _{2}\left(\mathrm{~N}_{\mathrm{x}}\right)$ & $3 \mathrm{~N}_{\mathrm{t}} \mathrm{N}_{\mathrm{x}}{ }^{2} \mathrm{~N}_{\mathrm{y}} \log _{2}\left(\mathrm{~N}_{\mathrm{x}}\right)$ \\
\hline FFT over $\mathrm{x}_{\mathrm{g}}$ & $3 \mathrm{~N}_{\mathrm{t}} \mathrm{N}_{\mathrm{x}}{ }^{2} \log _{2}\left(\mathrm{~N}_{\mathrm{x}}\right)$ & $3 \mathrm{~N}_{\mathrm{t}} \mathrm{N}_{\mathrm{x}}{ }^{2} \mathrm{~N}_{\mathrm{y}} \log _{2}\left(\mathrm{~N}_{\mathrm{x}}\right)$ \\
\hline FFT over $\mathrm{y}$ & - & $3 \mathrm{~N}_{\mathrm{t}} \mathrm{N}_{\mathrm{x}}{ }^{2} \mathrm{~N}_{\mathrm{y}} \log _{2}\left(\mathrm{~N}_{\mathrm{y}}\right)$ \\
\hline FFT over $\mathrm{t}$ & $3 \mathrm{~N}_{\mathrm{t}} \mathrm{N}_{\mathrm{x}}{ }^{2} \log _{2}\left(\mathrm{~N}_{\mathrm{t}}\right)$ & $3 \mathrm{~N}_{\mathrm{t}} \mathrm{N}_{\mathrm{x}}{ }^{2} \mathrm{~N}_{\mathrm{y}} \log _{2}\left(\mathrm{~N}_{\mathrm{t}}\right)$ \\
\hline Interpolation $\omega$ to $\omega_{0}$ & $\mathrm{M}_{\mathrm{interp}} \mathrm{N}_{\mathrm{t}} \mathrm{N}_{\mathrm{x}}{ }^{2}$ & $\mathrm{M}_{\text {interp }} \mathrm{N}_{\mathrm{t}} \mathrm{N}_{\mathrm{x}}{ }^{2} \mathrm{~N}_{\mathrm{y}}$ \\
\hline Kernel multiplication & $6 \mathrm{~N}_{\mathrm{t}} \mathrm{N}_{\mathrm{x}}{ }^{2}$ & $6 \mathrm{~N}_{\mathrm{t}} \mathrm{N}_{\mathrm{x}}{ }^{2} \mathrm{~N}_{\mathrm{y}}$ \\
\hline Summation over $\mathrm{k}_{\mathrm{xh}}$ & $2\left(\mathrm{~N}_{\mathrm{x}}-1\right)^{2} \mathrm{~N}_{\mathrm{t}}$ & $2\left(\mathrm{~N}_{\mathrm{x}}-1\right)^{2} \mathrm{~N}_{\mathrm{y}} \mathrm{N}_{\mathrm{t}}$ \\
\hline Iterate over each velocity values: & & \\
\hline \multicolumn{1}{|c|}{ Interpolation $\omega_{0}$ to $\mathrm{k}_{\mathrm{z}}$} & $\mathrm{M}_{\text {interp }}\left(2 \mathrm{~N}_{\mathrm{x}}-1\right) \mathrm{N}_{\mathrm{z}} \mathrm{N}_{\mathrm{e}}$ & $\mathrm{M}_{\text {interp }}\left(2 \mathrm{~N}_{\mathrm{x}}-1\right) \mathrm{N}_{\mathrm{y}} \mathrm{N}_{\mathrm{z}} \mathrm{N}_{\mathrm{e}}$ \\
\hline Kernel multiplication & $6\left(2 \mathrm{~N}_{\mathrm{x}}-1\right) \mathrm{N}_{\mathrm{z}} \mathrm{N}_{\mathrm{e}}$ & $6\left(2 \mathrm{~N}_{\mathrm{x}}-1\right) \mathrm{N}_{\mathrm{y}} \mathrm{N}_{\mathrm{z}} \mathrm{N}_{\mathrm{e}}$ \\
\hline
\end{tabular}




\begin{tabular}{|l|c|c|}
\hline IFFT over $\mathrm{k}_{\mathrm{x}}$ & $3\left(2 \mathrm{~N}_{\mathrm{x}}-1\right) \mathrm{N}_{\mathrm{z}} \mathrm{N}_{\mathrm{e}} \log _{2}\left(2 \mathrm{~N}_{\mathrm{x}}-1\right)$ & $3\left(2 \mathrm{~N}_{\mathrm{x}}-1\right) \mathrm{N}_{\mathrm{y}} \mathrm{N}_{\mathrm{z}} \mathrm{N}_{\mathrm{e}} \log _{2}\left(2 \mathrm{~N}_{\mathrm{x}}-1\right)$ \\
\hline IFFT over $\mathrm{k}_{\mathrm{y}}$ & - & $3\left(2 \mathrm{~N}_{\mathrm{x}}-1\right) \mathrm{N}_{\mathrm{y}} \mathrm{N}_{\mathrm{z}} \mathrm{N}_{\mathrm{e}} \log _{2}\left(\mathrm{~N}_{\mathrm{y}}\right)$ \\
\hline IFFT over $\mathrm{k}_{\mathrm{z}}$ & $3\left(2 \mathrm{~N}_{\mathrm{x}}-1\right) \mathrm{N}_{\mathrm{z}} \mathrm{N}_{\mathrm{e}} \log _{2}\left(\mathrm{~N}_{\mathrm{z}}\right)$ & $3\left(2 \mathrm{~N}_{\mathrm{x}}-1\right) \mathrm{N}_{\mathrm{y}} \mathrm{N}_{\mathrm{z}} \mathrm{N}_{\mathrm{e}} \log _{2}\left(\mathrm{~N}_{\mathrm{z}}\right)$ \\
\hline Varimax Calculation & $6\left(2 \mathrm{~N}_{\mathrm{x}}-1\right) \mathrm{N}_{\mathrm{z}} \mathrm{N}_{\mathrm{e}}$ & $6\left(2 \mathrm{~N}_{\mathrm{x}}-1\right) \mathrm{N}_{\mathrm{y}} \mathrm{N}_{\mathrm{z}} \mathrm{N}_{\mathrm{e}}$ \\
\hline
\end{tabular}

\subsection{Concluding Remarks}

In this chapter, we have presented the implementation of spatial diversity in UWB GPR by using MIMO configurations. The numerical experiments show that MIMO configurations can improve the imaging performance by providing higher resolution, lower side-lobe level, less image artifacts, higher SNR, and higher range of dip-angle.

The afore-mentioned advantages are evaluated by comparative study on several array configurations, i.e., SISO, SIMO, MIMO-half, and MIMO-all. Based on these results, we can conclude that MIMO-all configuration exhibits the best imaging performance, while MIMOhalf configuration has comparable quality with slightly higher side-lobe level. By contrast, SISO and SIMO arrays have lower resolution and higher side-lobe level. Moreover, in case of target with dipping surface, SIMO array yields distorted and unbalance image of the target. Despite of these drawbacks, SIMO array has the best efficiency in the number of components and the measurement process. Finally, the trade-off among image quality, system cost, and efficiency would be based on application needs.

We also investigate the impacts of migration methods on the imaging performance of SISO and MIMO arrays. In particular, 3 most common migration algorithms, namely Kirchhoff migration, phase-shift migration, and $\mathrm{f}-\mathrm{k}$ migration are evaluated on SISO and MIMO configurations. We observe that Kirchhoff migration has the best resolution and offers the best flexibility in sensor configuration and variation of wave velocity. However, this algorithm suffers from high side-lobe level. Meanwhile, phase-shift migration and f-k migration give advantages by lower side-lobe level and better computational speed, but at the expense of more restriction in sensor configuration and medium inhomogeneity. In addition, we have developed an equivalent phase-shift migration algorithm for SIMO configuration. This algorithm has comparable imaging quality to Kirchhoff migration with better computational speed.

The autofocusing method to handle MIMO configurations has been proposed. This algorithm utilizes the MZO operator, which transforms a MIMO data into its equivalent SISO data, and 
thus reduces the computational complexity in the optimization process. Based on performance analysis on simulation results and quantitative analysis of computational complexities, the proposed algorithm can significantly reduce the computation time while preserve the imaging capability of MIMO configurations. 


\section{CHAPTER 4}

\section{The Waveform Diversity for UWB GPR Systems}

Waveform diversity has been proved as a new concept to improve the performance of communication and radar systems. In this chapter, we present the implementation of the proposed waveform diversity for UWB GPR. Several theories to support the proposed waveform diversity method are introduced in Section 4.1. In Section 4.2, we propose a processing method that utilizes the waveform diversity information to improve imaging capability and perform target characterization. The performance of this method on several target shapes and materials are investigated in Section 4.3. Finally, the chapter is summarized in Section 4.4.

\subsection{Review of Existing Theory}

\subsubsection{Waveform Diversity in Radar System}

Waveform diversity has become an attractive approach to be explored in several applications of radar system. This concept was originated from communication system, where various waveform design techniques were developed to enhance the system performance on particular application [62], [63].

In narrow-band radar, waveform diversity holds a necessary role for the implementation of multi-input multi-output (MIMO) concept. The MIMO system in this case comprises several transmitters. Each transmitter emits a unique sequence of waveforms with particular coding schemes [59]. It has been demonstrated that waveform diversity can increase the maximum number of target that can be identified, enable the direct applicability of adaptive technique, and improve the flexibility of transmit beam-pattern design.

Another implementation of waveform diversity is in radar for tracking purpose. Based on a study of bat behavior in [64], it was found that such animal uses waveform diversity with frequency modulated waveform to locate its prey. Inspired by this behavior, in [65] Wang et al. adopted the corresponding waveform diversity approach for tracking algorithm of radar system. Several waveforms were selected adaptively to minimize the tracking error [109]. 
The implementations of waveform diversity for imaging radar have been proposed in [66][68], which are mainly developed for synthetic aperture radar (SAR) system. These techniques apply a coding scheme to the chirp rate of frequency modulated continuous wave (FMCW) waveform in order to obtain orthogonal transmission channels. It was demonstrated that the proposed methods provide waveform with large time-bandwidth product, reduce the range/Doppler ambiguities, and improve the performance in ground moving target indication (GMTI).

While the aforementioned examples are for narrow-band system, a number of works also have been carried out to study waveform diversity for UWB system. Several waveform sets, such as Hermite polynomial and Gegenbauer, have been suggested for multi-access technique in UWB communication system. In [70] and [80], these waveform sets were examined based on some aspects, such as the compliance with FCC frequency emission restrictions, the bit error rate (BER) performance, and the possibility in implementing multiuser access techniques. These waveform sets were also investigated for collision avoidance radar system in [110]. In particular, the study showed that Gegenbauer waveforms and Hermite polynomial waveforms exhibit comparable performance in terms of resolution and side-lobe ratio. However, the spectrum of Hermite polynomial is more confined than Gegenbauer, so we decide to use Hermite polynomial waveforms in the implementation of waveform diversity for UWB GPR.

In Hermite polynomial waveform sets, each waveform occupies different frequency range. This frequency range is shifted up as the waveform order increases. On the other hand, the target interaction with the incident wave is affected by the spectrum of the transmitted waveform. According to the scattering theory [103], this interaction can be classified into 2 cases. The first case occurs when the target has small dielectric contrast compared to background medium, in which the incident wave penetrates through the target. By contrast, in the second case the wave interacts only at the target surface, which typically holds for conductor target or target with high dielectric contrast. Therefore, before going into the detailed formulation of the proposed waveform diversity method, we present in the following a review of scattering theory and formulations of the scattered wave. 


\subsubsection{Scattering Theory}

In the scattering problem, a wave that was radiated by a primary source propagates through physical obstacle or inhomogeneity in the medium of propagation. Secondary sources are created by the interaction between the incident wave and the obstacle. Based on the interaction between the object and the wave, there are 2 types of scattering problem: scattering from penetrable target where the incident wave penetrates into the interior of the obstacle, and scattering from non-penetrable target where the interaction of the incident wave with the obstacle takes place only over the object surface.

The penetrable object case is applicable for obstacle with small inhomogeneity compared to the background medium. This inhomogeneity can be modelled as a scattering potential given in (4.1), where $V(\boldsymbol{r})$ is the scattering potential, $k_{0}$ is the constant wavenumber of the background, $n(\boldsymbol{r}, \omega)$ is the complex index of refraction of the scattering object, and $n_{0}(\omega)$ is the complex index of refraction of the background medium.

$$
V(\mathbf{r})=k_{0}{ }^{2}\left[1-\left(\frac{n(\mathbf{r}, \omega)}{n_{0}(\omega)}\right)^{2}\right]
$$

The resulting scattered wave is given by Lippmann-Schwinger (LS) integral equation in (4.2), where $U(\boldsymbol{r}, \omega)$ denotes the total wave, $U^{(s)}(\boldsymbol{r}, \omega)$ denotes the scattered wave, $R$ is the distance from $\boldsymbol{r}$ to $\boldsymbol{r}^{\prime}$, and $G_{0+}\left(\boldsymbol{r}-\boldsymbol{r}^{\prime}\right)$ is the outgoing-wave Green function given by (4.3).

$$
\begin{gathered}
U^{(s)}(\mathbf{r}, \omega)=\int d^{3} \mathbf{r}^{\prime} G_{0+}\left(\mathbf{r}-\mathbf{r}^{\prime}\right) V\left(\mathbf{r}^{\prime}\right) U\left(\mathbf{r}^{\prime}, \omega\right) \\
G_{0+}\left(\mathbf{r}-\mathbf{r}^{\prime}\right)=\frac{1}{4 \pi} \frac{e^{i k_{0} R}}{R}
\end{gathered}
$$

A simplification of this equation is well-known as Born's approximation. Such approach assumes that the inhomogeneity is small, so the total wave $U(\boldsymbol{r}, \omega)$ can be approximated by the incident wave $U^{(i n)}(\boldsymbol{r}, \omega)$, and the scattered wave in (4.2) becomes

$$
U_{B}^{(s)}(\mathbf{r}, \omega)=\int d^{3} \mathbf{r}^{\prime} G_{0+}\left(\mathbf{r}-\mathbf{r}^{\prime}\right) V\left(\mathbf{r}^{\prime}\right) U^{(i n)}\left(\mathbf{r}^{\prime}, \omega\right)
$$

By contrast, the non-penetrable object case occurs on target with high dielectric constant value or conductor target. Such case can be formulated by using boundary condition equation. There are 2 types of boundary conditions, namely Neumann and Dirichlet boundary conditions. In Neumann boundary condition, the normal derivative of the field vanishes over 
the object surface, while in Dirichlet boundary condition the field vanishes over the scatterer surface. The scattered wave of Neumann and Dirichlet boundary conditions can be formulated as in (4.5) and (4.6) respectively, where $\boldsymbol{n}$ ' is the normal vector to the surface and $\partial \tau_{0}$ is the integration domain of the surface.

$$
\begin{aligned}
U^{(s)}(\mathbf{r}, \omega) & =-\int_{\partial \tau_{0}} d S^{\prime} U\left(\mathbf{r}^{\prime}, \omega\right) \frac{\partial}{\partial n^{\prime}} G_{0+}\left(\mathbf{r}-\mathbf{r}^{\prime}\right) \\
U^{(s)}(\mathbf{r}, \omega) & =\int_{\partial \tau_{0}} d S^{\prime} G_{0+}\left(\mathbf{r}-\mathbf{r}^{\prime}\right) \frac{\partial}{\partial n^{\prime}} U\left(\mathbf{r}^{\prime}, \omega\right)
\end{aligned}
$$

Since subsurface objects are typically made of conductor or high dielectric materials, we only consider the Dirichlet boundary condition. Similar to the penetrable object case, we can further simplify (4.6) by using Physical-Optic (PO) approximation. This approximation assumes that the target has a convex surface that varies slowly relative to the wavelength of the incident wave as illustrated in Fig. 4.1. Thus, some regions in the target face the incident wave (lit region), while some other regions are blocked (unlit region).

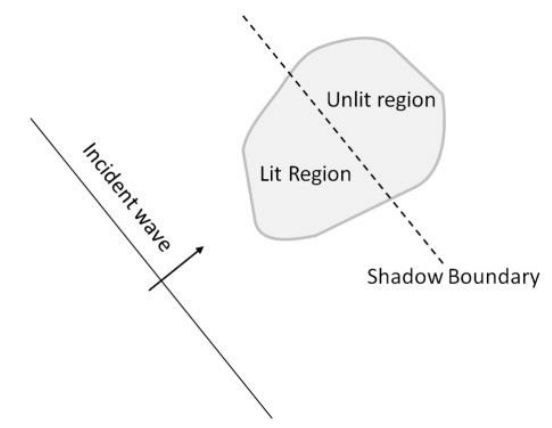

Fig. 4.1: Convex surface of the target.

The approximation is performed by taking into account only the lit region. Specifically, we carry out the integration only over lit region $\left(\partial \tau_{0 l}\right)$ and approximate $U(\boldsymbol{r}, \omega)$ with $U^{(i n)}<(\boldsymbol{r}, \omega)$, which is the part of $U^{(i n)}(\boldsymbol{r}, \omega)$ that propagates toward the object. The result is given in (4.7).

$$
U_{P O}^{(s)}(\mathbf{r}, \omega)=2 \int_{\partial \tau_{0 l}} d S^{\prime} G_{0+}\left(\mathbf{r}-\mathbf{r}^{\prime}\right) \frac{\partial}{\partial n^{\prime}} U_{<}^{(i n)}\left(\mathbf{r}^{\prime}, \omega\right)
$$

In our case, we consider an impulse GPR in which the transmitted waveform is a bandlimited impulse. Thus, the incident wave can be expressed by (4.8), where $F(\omega)$ is the spectrum the impulse waveform. 


$$
U^{(i n)}(\mathbf{r}, \omega)=\frac{F(\omega)}{4 \pi} \frac{e^{i k_{0} R}}{R}
$$

Based on (4.3) and (4.8), we can foresee that the derivative of $G_{0+}\left(\boldsymbol{r}-\boldsymbol{r}^{\prime}\right)$ and $U^{(i n)}(\boldsymbol{r}, \omega)$ are proportional to $\exp \left(i k_{0} R\right)$. Thus, by using those equations, one can show that Born's approximation and PO approximation can be expressed in general by (4.9), where $R\left(\boldsymbol{r}^{\prime}\right)$ is the target region and each approximation has different value of kernel $A_{l}$.

$$
U^{(s)}(\mathbf{r}, \omega)=\int d^{3} \mathbf{r}^{\prime} R\left(\mathbf{r}^{\prime}\right) A_{1}\left(\mathbf{r}, \mathbf{r}^{\prime}, \omega\right) F(\omega) e^{i 2 k_{0} R}
$$

The kernel $A_{l}$ in (4.9) is a function of $\omega$, which implies that the frequency responses of these approximations are distinct from each other. Therefore, we may expect that the penetrable and the non-penetrable targets produce different responses over each waveform. Furthermore, we can exploit such properties to perform target classification based on its material.

\subsubsection{Support Vector Machine}

As mentioned earlier, we will utilize waveform diversity data to perform classification on target material. Such classification is carried out by using support vector machine (SVM) classifier, which has been widely applied in numerous problems such as face recognition, text classification, and etc.

SVM is originally developed as a binary classification technique. The main concept of SVM is to obtain an optimum hyper-plane that can separate all samples between the two classes. This can be illustrated by Fig. 4.2 [111] for linear classification. The hyper-plane for linear case can be formulated by (4.10), where $\boldsymbol{x}$ is a feature vector, $\boldsymbol{w}$ is a weight vector, and $b$ is a bias.

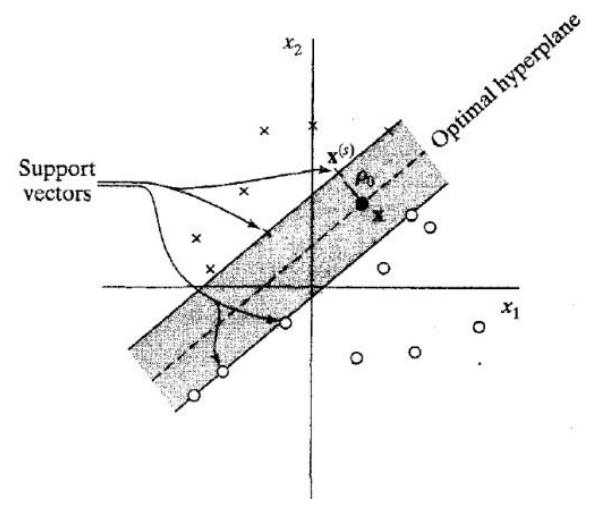

Fig. 4.2: Illustration of an optimum hyperplane. 


$$
\mathbf{w}^{T} \mathbf{x}+b=0
$$

Margin of separation is defined as the distance between the hyper-plane and the closest data point. The hyper-plane is said to be optimum if the margin of separation between the two classes is maximized. It can be shown that margin separation $(\rho)$ is proportional to inverse of Euclidian norm of weight vector $\boldsymbol{w}$ as given in (4.11), and thereby the maximization of $\rho$ is equivalent to the minimization of $\|w\|$.

$$
\rho=\frac{2}{\|\mathbf{w}\|}
$$

Based on such properties, we can formulate a constrained optimization problem as follows. Given $N$ training samples with $i$-th feature vector $\boldsymbol{x}_{i}$ and the corresponding class $d_{i}$, minimize the cost function

$$
\Phi(\mathbf{w})=\frac{1}{2} \mathbf{w}^{T} \mathbf{w}
$$

with respect to constraints

$$
d_{i}\left(\mathbf{w}^{T} \mathbf{x}_{i}+b\right) \geq 1 \quad \text { for } i=1,2, \ldots, N
$$

This optimization problem can be solved by using Lagrange multipliers technique, in which the Langrangian function is expressed by (4.14) with $\alpha_{i}$ as the Langrange multipliers.

$$
J(\mathbf{w}, b, \alpha)=\frac{1}{2} \mathbf{w}^{T} \mathbf{w}-\sum_{i=1}^{N} \alpha_{i}\left[d_{i}\left(\mathbf{w}^{T} \mathbf{x}_{i}+b\right)-1\right]
$$

This equation can be simplified by constructing its dual problem based on duality theorem. By such method, the objective function in (4.14) is translated into (4.15), while the corresponding constraints are given in (4.16). As we can see, the optimization now only depends on the Langrange multiplier $\alpha_{i}$, compared to (4.14) which depends on $w$ and $b$ in addition to $\alpha_{i}$. This dual problem can be solved by using quadratic optimization.

$$
\begin{aligned}
& Q(\alpha)=\sum_{i=1}^{N} \alpha_{i}-\frac{1}{2} \sum_{i=1}^{N} \sum_{j=1}^{N} \alpha_{i} \alpha_{j} d_{i} d_{j} \mathbf{x}_{i}^{T} \mathbf{x}_{j} \\
& \text { - } \quad \sum_{i=1}^{N} \alpha_{i} d_{i} \\
& \text { - } \quad \alpha_{i} \geq 0 \quad \text { for } i=1,2, \ldots, N
\end{aligned}
$$


The case that we have introduced so far is based on separable patterns assumption. Practically, such properties do not always hold. To extend the method on non-separable case, the constraints in (4.13) can be relaxed into (4.17). In this equation, $\xi_{i}$ is so-called as slack variable, which is a measure of deviation of the data point from the correct region.

$$
d_{i}\left(\mathbf{w}^{T} \mathbf{x}_{i}+b\right) \geq 1-\xi_{i} \quad \text { for } i=1,2, \ldots, N
$$

The new cost function that accounts such error is formulated by (4.18), where $C$ is a parameter to control the trade-off between the computational complexity and the number of non-separable points. This parameter can be determined by using cross-validation test [111].

$$
\Phi(\mathbf{w}, \xi)=\frac{1}{2} \mathbf{w}^{T} \mathbf{w}+C \sum_{i=1}^{N} \xi_{i}
$$

The formulation of dual problem for cost function in (4.18) is given by (4.19) with constraints given by (4.20). We can observe that the difference of separable and nonseparable cases is only on the parameter $C$, which confines the maximum value of $\alpha_{i}$.

$$
\begin{aligned}
& Q(\alpha)=\sum_{i=1}^{N} \alpha_{i}-\frac{1}{2} \sum_{i=1}^{N} \sum_{j=1}^{N} \alpha_{i} \alpha_{j} d_{i} d_{j} \mathbf{x}_{i}^{T} \mathbf{x}_{j} \\
& \text { - } \quad \sum_{i=1}^{N} \alpha_{i} d_{i} \\
& \text { - } \quad 0 \leq \alpha_{i} \leq C \quad \text { for } i=1,2, \ldots, N
\end{aligned}
$$

We can generalize the previous methods into non-linear case by simply introducing nonlinear vector function $\boldsymbol{\varphi}\left(\boldsymbol{x}_{i}\right)=\left[\varphi_{0}\left(\boldsymbol{x}_{i}\right), \varphi_{1}\left(\boldsymbol{x}_{i}\right), \ldots, \varphi_{m l}\left(\boldsymbol{x}_{i}\right)\right]^{T}$ that transforms the original feature vector into a higher dimensional space $m_{l}$. Thus, we can reformulate the dual optimization problem in (4.19) for non-linear case by replacing $\mathbf{x}_{\mathbf{i}}$ with $\boldsymbol{\varphi}\left(\mathbf{x}_{\mathbf{i}}\right)$ and obtain

$$
Q(\alpha)=\sum_{i=1}^{N} \alpha_{i}-\frac{1}{2} \sum_{i=1}^{N} \sum_{j=1}^{N} \alpha_{i} \alpha_{j} d_{i} d_{j} \boldsymbol{\varphi}\left(\mathbf{x}_{i}\right)^{T} \boldsymbol{\varphi}\left(\mathbf{x}_{j}\right)
$$

Although the concept of vector function $\boldsymbol{\varphi}\left(\mathbf{x}_{\mathbf{i}}\right)$ seems to be obvious, in practice it is difficult to determine the suitable function for a given dataset. Moreover, the dimension of such function can be tremendously high, so the calculation becomes a daunting task. These shortcomings can be resolved by using Mercer's theorem which states that the calculation of inner product of $\boldsymbol{\varphi}\left(\mathbf{x}_{\mathbf{i}}\right)$ can be replaced by the calculation of inner-product kernel 


$$
K\left(\mathbf{x}, \mathbf{x}_{i}\right)=\varphi^{T}(\mathbf{x}) \varphi\left(\mathbf{x}_{i}\right)
$$

Some of the most common functions for inner-product kernel in SVM are given in Table 4.1. In our case, we will use the radial-basis function network kernel.

Table 4.1: KERNEL FUNCTIONS IN SVM

\begin{tabular}{|l|c|}
\hline \multicolumn{1}{|c|}{ Type of SVM } & Inner-product kernel $\boldsymbol{K}\left(\boldsymbol{x}, \boldsymbol{x}_{i}\right)$ \\
\hline Polynomial learning machine & $\left(\mathbf{x}^{T} \mathbf{x}_{i}+1\right)^{p}$ \\
\hline Radial-basis function network & $\exp \left(-\frac{1}{2 \sigma^{2}}\left\|\mathbf{x}-\mathbf{x}_{i}\right\|^{2}\right)$ \\
\hline Two-layer perceptron & $\tanh \left(\beta_{0} \mathbf{x}^{T} \mathbf{x}_{i}+\beta_{1}\right)$ \\
\hline
\end{tabular}

Following this approach, the dual problem in (4.21) can be simplified into (4.23) with similar constraints.

$$
Q(\alpha)=\sum_{i=1}^{N} \alpha_{i}-\frac{1}{2} \sum_{i=1}^{N} \sum_{j=1}^{N} \alpha_{i} \alpha_{j} d_{i} d_{j} K\left(\mathbf{x}_{i}, \mathbf{x}_{j}\right)
$$

Finally, the optimum hyper-plane in (4.10) can be determined by

$$
\sum_{i=1}^{N} \alpha_{i} d_{i} K\left(\mathbf{x}, \mathbf{x}_{i}\right)=0
$$

\subsection{Proposed Waveform Diversity Method for UWB GPR}

In Chapter 3, we have introduced and analyzed several sensor configurations for UWB GPR. However, in the study of waveform diversity, we utilize only SISO measurement. The system is illustrated in Fig. 4.3, where a pair of transmitter and receiver is lifted at height $10 \mathrm{~cm}$ above the ground surface. This sensor will be moved step-by-step along $x$-axis over the scan area with spatial sampling $1.5 \mathrm{~cm}$. The data is collected with several types of waveform at different time instances.

$\square$ Movement direction

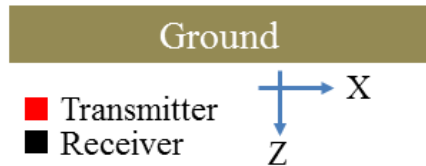

Fig. 4.3: Sensor configuration on waveform diversity. 
As discussed earlier, we choose the modified Hermite polynomial (HP) waveforms set to implement waveform diversity on GPR system. We employ the first 4 order of this waveform set, which can be formulated as in (4.25). The pulse widths are adjusted to be $1 \mathrm{~ns}$.

$$
\begin{aligned}
& h_{0}(t)=\exp \left(-t^{2} / 4\right) \\
& h_{1}(t)=t \exp \left(-t^{2} / 4\right) \\
& h_{2}(t)=\left(t^{2}-1\right) \exp \left(-t^{2} / 4\right) \\
& h_{3}(t)=\left(t^{3}-3 t\right) \exp \left(-t^{2} / 4\right)
\end{aligned}
$$

These waveforms and their corresponding spectrums are given in Fig. 4.4.

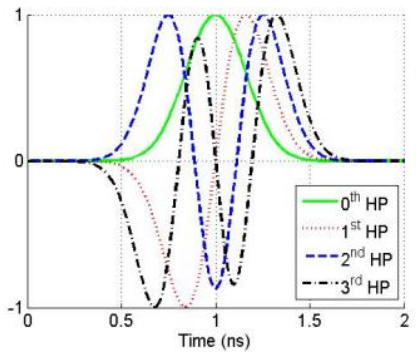

(a) Time domain

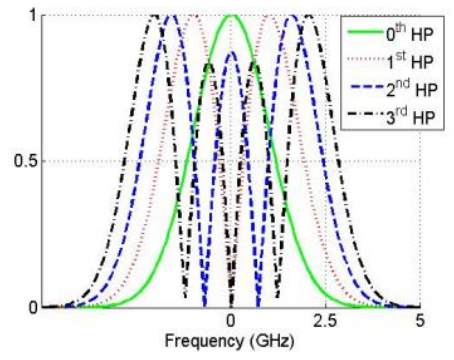

(b) Frequency domain

Fig. 4.4: Original Hermite polynomial waveforms and spectrums.

However, in real experiment, the transmitted waveforms experience distortion due to the finite system bandwidth. Similar to Chapter 3, this study is conducted based on simulation data from the full electromagnetic field simulator GPRMax. Fig. 4.5 shows the resulting waveforms after generated by GPRMax simulation in time domain and in frequency domain. By comparing the results with Fig. 4.4, we can observe the transformation on the shapes of the waveform in time domain, while in frequency domain some low frequency components are eliminated.

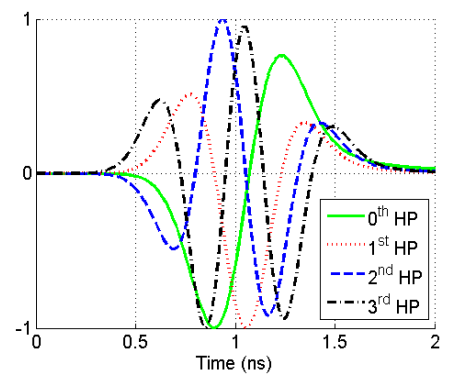

(a) Time domain

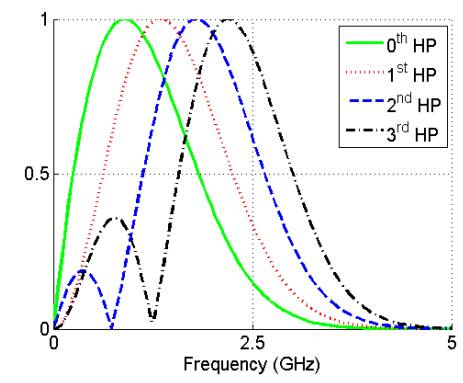

(b) Frequency domain

Fig. 4.5: Hemite polynomial waveforms and spectrums in GPRMax simulation. 
Notice that the spectrum range in Fig. 4.5(b) can also be covered by SFCW waveform. However, we decide to focus on impulse GPR based on some rationales. Firstly, impulse GPR outperforms SFCW GPR in term of data acquisition speed. Secondly, we perform study on simulation data generated by GPRMax, which is based on FDTD solution of Maxwell equations in time domain. Thus, it is preferable to use impulse waveform, which also provides measurement data in time domain. Nevertheless, the proposed waveform diversity methods in this chapter still can be generalized for SFCW waveform.

The signal processing steps are given in Fig. 4.6. The first step is preprocessing, which is purposed to enhance the SNR and reduce the clutter in the raw data. Since we use simulation data that have ideal condition, we only perform background removal and DC offset removal for this part. In the next step, we separate the processing into 2 different parts. In the left part, we apply a particular filtering method to combine the data from all waveforms and obtain single image. Meanwhile, in the right part we continue processing each waveform independently by using f-k migration, followed by classification on target material. Finally, we combine the image from the left part with the classification information from the right part. In the following subsections, the filtering method to combine all the waveforms and the target classification method are discussed in detail.

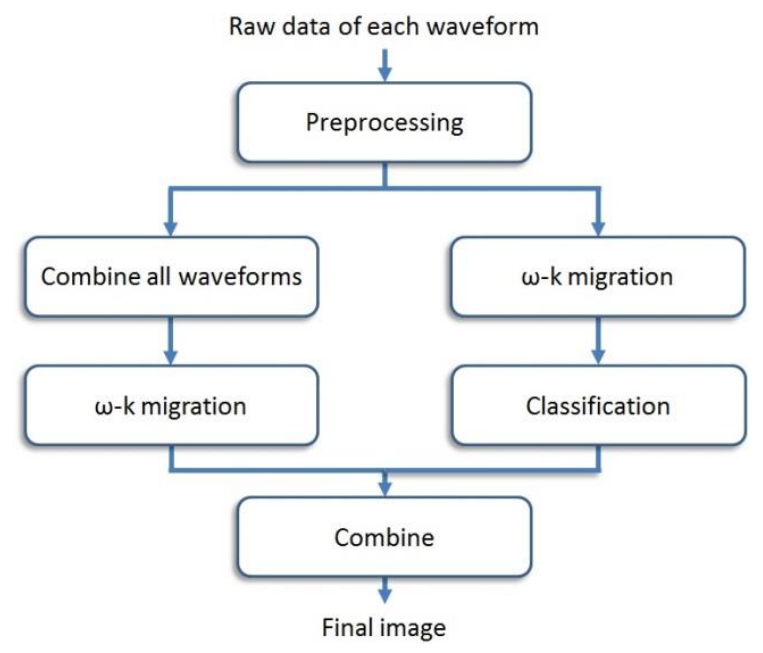

Fig. 4.6: Signal processing of the GPR data.

\subsubsection{Filtering Method to Combine All Waveforms}

We formulate the filtering technique based on the spectrum of the corresponding waveform as illustrated in Fig. 4.7. Firstly, the data are converted into frequency and wavenumber domain. Then, we apply filtering on each waveform independently, which is implemented by 
multiplication in $\left(k_{x}, \omega\right)$ domain. Finally, we sum all these data and pass them into the rest of the migration step.

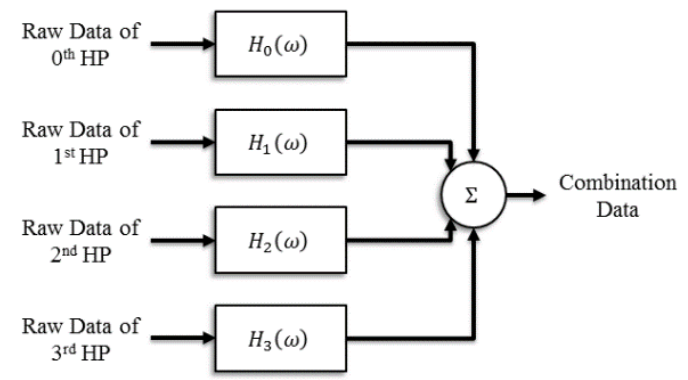

Fig. 4.7: Diagram of the filtering method to combine all waveforms.

The next problem is how to determine the filter response. Since our main goal is to obtain a wider bandwidth and a constant amplitude spectrum, the filtering result must satisfy

$$
\sum_{i=0}^{3} H_{i}(\omega) F_{i}(\omega)=F_{T}(\omega)
$$

In (4.26), $F_{i}(\omega)$ denotes the spectrum of the $i$-th waveform, while $F_{T}(\omega)$ denotes the total desired spectrum in the combination result. Moreover, we expect that the data with small amplitude is not excessively amplified to avoid the degradation in SNR. Therefore, we set the second requirement as

$$
\frac{H_{i}(\omega) F_{i}(\omega)}{H_{j}(\omega) F_{j}(\omega)}=\frac{F_{i}(\omega)}{F_{j}(\omega)}
$$

By solving (4.26) and (4.27), we get the solution of the filter as

$$
H_{i}(\omega)=\frac{F_{T}(\omega)}{\sum_{j=0}^{3} F_{j}(\omega)}
$$

In order to predict the possible advantages of such combination approach, we perform theoretical analysis on the final migration result. Suppose we have an arbitrary waveform with spectrum $F(\omega)$. As described in Subsection 4.1.2, the scattered wave for both penetrable target and non-penetrable target can be formulated as

$$
U^{(s)}\left(x_{0}, \omega\right)=\int d^{2} \mathbf{r}^{\prime} R\left(\mathbf{r}^{\prime}\right) A_{1}\left(x_{0}, \mathbf{r}^{\prime}, k\right) F(\omega) e^{i 2 k R}
$$


In practice, there is a limitation on the effective range of the measurement over $x$-axis, so we need to modify (4.29) by introducing additional factor $A_{2}\left(x_{0}\right)$ given in (4.30). This function indicates that the data is available only over certain range of $x_{0}$, where $x_{L}$ and $L_{x}$ are the center point and the length of such effective range respectively.

$$
A_{2}\left(x_{0}\right)=\operatorname{rect}\left(\frac{x_{0}-x_{L}}{L_{x}}\right)
$$

By multiplying (4.29) with $A_{2}\left(x_{0}\right)$, we obtain the scattered wave equation as

$$
U^{(s)}\left(x_{0}, \omega\right)=\int d^{2} \mathbf{r}^{\prime} R\left(\mathbf{r}^{\prime}\right) A_{1}\left(x_{0}, \mathbf{r}^{\prime}, k\right) A_{2}\left(x_{0}\right) F(\omega) e^{i 2 k R}
$$

The first step of $\mathrm{f}-\mathrm{k}$ migration is the Fourier transform on $x_{0}$ and $t$. Since (4.31) is already in the $\omega$ domain, we only need to perform this operation on $x_{0}$. By performing Fourier transform and applying method of stationary phase (MOSP) approximation on (4.31), we obtain

$$
U^{(s)}\left(k_{x}, \omega\right)=\int d^{2} \mathbf{r}^{\prime} R\left(\mathbf{r}^{\prime}\right) F(\omega) A_{1}{ }^{\prime} A_{2}{ }^{\prime} A_{3} e^{-i \mathbf{k} \cdot \mathbf{r}^{\prime}}
$$

where $A_{l}$ ' is the transformation result of $A_{l}$ by the MOSP approximation, and

$$
A_{2}{ }^{\prime}=\operatorname{rect}\left[\frac{\frac{k_{x}}{k_{z}}-\left(\frac{x^{\prime}-x_{L}}{z^{\prime}}\right)}{\left(L_{x} / z\right)}\right], A_{3}=\operatorname{rect}\left[\frac{k_{x}}{(2 \pi / d x)}\right], \mathbf{k}=\left(k_{x}, k_{z}\right), k_{z}=-\frac{2 \omega}{c} \sqrt{1-\left(\frac{c k_{x}}{2 \omega}\right)^{4}} .
$$

Moreover, an additional factor $A_{3}$ arises in (4.32) as an implication of finite spatial sampling $d x$ in the measurement. The final step is the inverse Fourier transform on $\left(k_{x}, k_{z}\right)$. The result is given in (4.33).

$$
U(\mathbf{r})=\int d^{2} \mathbf{k} d^{2} \mathbf{r}^{\prime} R\left(\mathbf{r}^{\prime}\right) F(\omega) A_{1}^{\prime} A_{2}{ }^{\prime} A_{3} e^{i \mathbf{k} \cdot\left(\mathbf{r}-\mathbf{r}^{\prime}\right)}
$$

From (4.33), we can observe 3 main factors that confine the integration range over the wavenumber domain $\boldsymbol{k}$ :

1) The waveform spectrum $F(\omega)$ limits the integration range in circular manner. This constraint will appear as a doughnut-like shape as shown in Fig. 4.8(a), where $\omega_{\min }$ and $\omega_{\max }$ are the minimum and maximum frequencies in the spectrum of the waveform. 
2) The aperture length factor $A_{2}$ ' restricts the maximum value of $\tan \alpha=k_{x} / k_{z}$ as shown in Fig. 4.8(b), where $\alpha$ is equivalent to the dip-angle range of the target.

3) The sampling distance factor $A_{3}$ confines the value of $k_{x}$ to be within $[-\pi / d x, \pi / d x]$ as shown in Fig. 4.8(c).

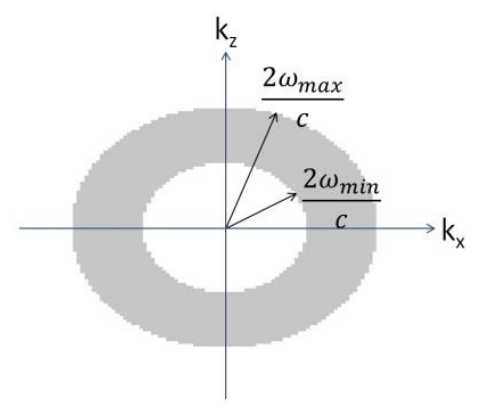

(a) Waveform spectrum

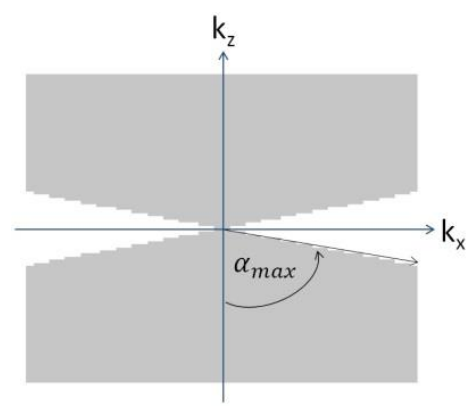

(b) Aperture length

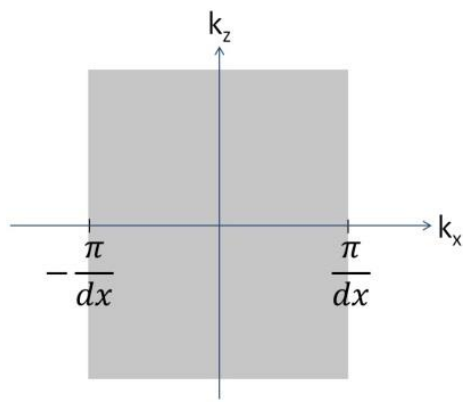

(c) Finite sampling distance

Fig. 4.8: Effect of several measurement factors on integration range in wavenumber domain.

By combining these factors, we can obtain the integration range of each HP waveform in wavenumber domain as given in Fig. 4.9.

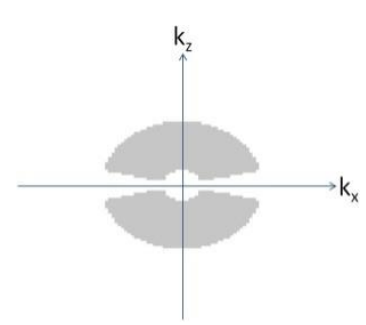

(a) $0^{\text {th }}$-order HP

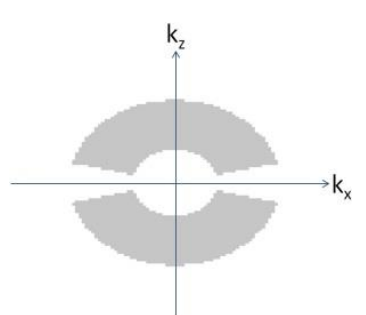

(b) $1^{\text {st }}$-order HP

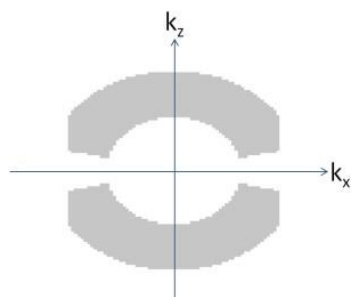

(c) $2^{\text {nd }}$-order HP

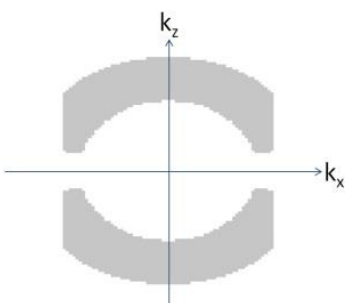

(d) $3^{\text {rd }}$-order HP

Fig. 4.9: Integration range of each waveform in wavenumber domain.

The proposed filtering method combines all these waveforms in frequency and wavenumber domain, and thus the integration domain covers all the ranges presented in Fig. 4.9. Based on such properties, we can predict the advantages of this combination method as follows:

1) Improvement on resolution

Based on (4.33), the response of the waveform on point target is simply the inverse Fourier transform of $A_{l}$ ' over the wavenumber domain given in Fig. 4.9. If the variation of $A_{l}$ ' over the integration region is omitted, the resolution at direction $\hat{\mathbf{k}}$ can be approximated by 


$$
\delta_{\hat{k}} \approx \frac{1}{B W(\hat{\mathbf{k}})}
$$

where $B W(\hat{\mathbf{k}})$ is the integration range at direction given by unit vector $\hat{\mathbf{k}}$. Since the proposed method combines all the domain of $\boldsymbol{k}$ from each waveform, we obtain wider integration range $B W(\hat{\mathbf{k}})$ in any direction of $\hat{\mathbf{k}}$, and thus a better resolution.

2) Improvement on dip-angle range

The direction of wavenumber unit vector $\hat{\mathbf{k}}$ shows the direction of propagation of the wave. Let $\hat{\mathbf{n}}$ denotes the normal vector of target surface. According to Snell's law, the mono-static sensor can successfully receive the target reflection if the wave satisfies (4.35).

$$
\hat{\mathbf{k}}=\hat{\mathbf{n}}
$$

This equation implies that a surface can be imaged if its dip angle is perpendicular to the angle of $\hat{\mathbf{k}}$. In Fig. 4.9, we can see that the lower order HP pulses provide better coverage over lower angle of $\hat{\mathbf{k}}$. Meanwhile, the third-order HP pulse has nonuniform range at the lower angle region, which reduces its ability in imaging surface with high inclination angle. The combination of all these domains leads to a better coverage of $\hat{\mathbf{k}}$, and therefore improves the imaging capability over various dip-angles.

\subsubsection{Target Classification Method}

In Subsection 4.2.1, we have demonstrated theoretically that combining all waveforms might improve the imaging capability. However, each waveform may possess additional information regarding the target, which can be exploited to characterize the target. From the scattering theory introduced in Subsection 4.1.2, we have seen that penetrable and nonpenetrable objects yield different kernel $A_{l}$, which is a function of $\left(k_{x}, k_{z}\right)$. On the other hand, Fig. 4.9 shows that each waveform occupies different region in wavenumber domain. Therefore, we can expect these waveforms to produce distinct characteristics on penetrable and non-penetrable targets.

To verify such properties, we analyze the imaging result of each waveform over various target shapes and materials by using simulation data. The simulation scenarios are given in 
Fig. 4.10. In the first scheme, we simulate a cylinder target in some values of diameter: $1 \mathrm{~cm}$, $2 \mathrm{~cm}$, and $3 \mathrm{~cm}$. In the second scheme, we have a plate with thickness of $1 \mathrm{~cm}$, width of 20 $\mathrm{cm}$, and several values of inclination angle $\theta$ ranging from $0^{\circ}$ to $45^{\circ}$. We conduct each simulation on 3 different target materials: low dielectric constant material $\left(\varepsilon_{r}=3.5, \mu_{r}=1, \sigma\right.$ $=0 \mathrm{~S} / \mathrm{m})$, high dielectric constant material $\left(\varepsilon_{r}=72, \mu_{r}=1, \sigma=4 \mathrm{~S} / \mathrm{m}\right)$, and perfect electric conductor (PEC). In all these simulations, the subsurface is homogeneous with relative permittivity of 6 , relative permeability of 1 , and conductivity of $0.001 \mathrm{~S} / \mathrm{m}$.

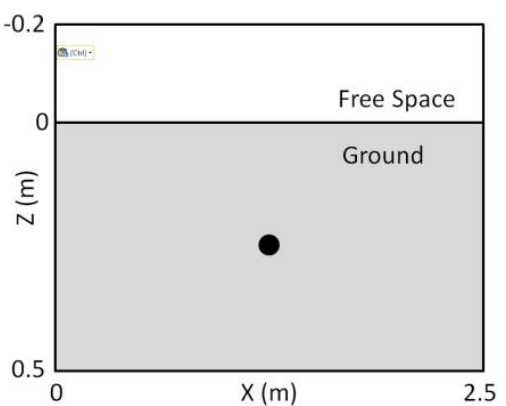

(a) Cylinder target

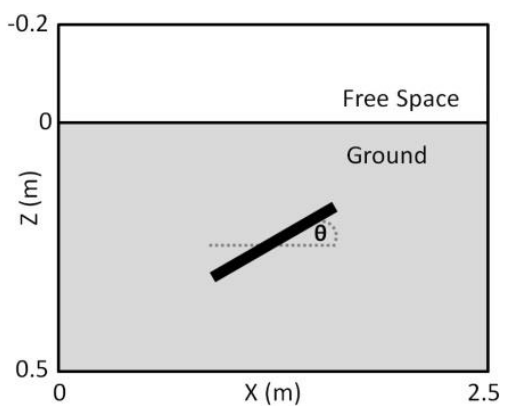

(b) Plate target

Fig. 4.10: Simulation configurations on several target materials.

The most obvious characteristic to observe from each waveform is the amplitude of the resulting image. Therefore, after applying f-k migration to the simulation results, we calculate the ratios of amplitude between each waveform over the target region. Specifically, the ratios of image from $1^{\text {st }}$ order HP, $2^{\text {nd }}$ order HP, and $3^{\text {rd }}$ order HP waveforms are taken with respect to the image of $0^{\text {th }}$ order HP waveform. The results are presented in Fig. 4.11 for each combination of amplitude ratios.

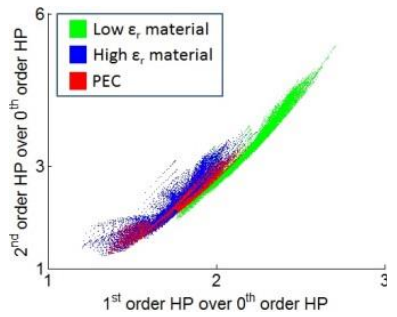

(a) $1^{\text {st }}$ and $2^{\text {nd }}$ order $\mathrm{HP}$

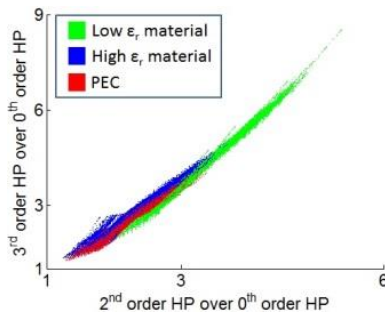

(b) $2^{\text {nd }}$ and $3^{\text {rd }}$ order HP

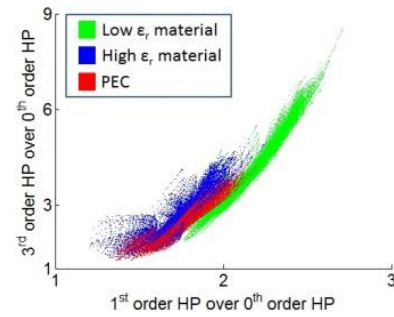

(c) $1^{\text {st }}$ and $3^{\text {rd }}$ order HP

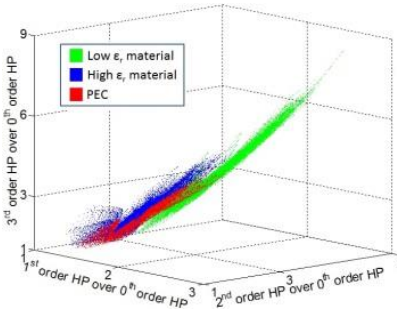

(d) $1^{\text {st }}, 2^{\text {nd }}$, and $3^{\text {rd }}$ order HP

Fig. 4.11: Combination of amplitude ratios of $1^{\text {st }}, 2^{\text {nd }}$, and $3^{\text {rd }}$ order HP over $0^{\text {th }}$ order HP.

We can observe in Fig. 4.11 that the penetrable target which made of low dielectric constant material, and the non-penetrable target which made of high dielectric constant and PEC materials, tend to have different amplitude ratios. Despite some samples from different 
material are overlapped, overall they can be separated by a particular boundary. We can make use these properties to perform simple classification on these two types of target.

A number of GPR target classification approaches based on material have been developed in the past. In [112], the waveform of the target reflection in raw data is used as feature vector supplied to SVM classifier. Following the similar way, [113], [114], and [115] suggest several variants of feature vectors such as spectrum of the A-scan, fast and low decay points in the spectrum, statistics of the signal, and discrete cosine transform of the A-scan. Another work in [116] employs multilayer perceptron to perform classification. Although it has been demonstrated that these methods can achieve high classification accuracy, most of the training scenarios might not be available in real case. Moreover, these features are extracted from the A-scan of the data without migration process, and therefore the resolving capability might be lower than using the migrated image.

To overcome these issues, we propose a target classification method that based on limited training scenarios which are more feasible to produce in practice. In contrast to the existing classification methods, the proposed technique is carried out on the migrated data of each waveform, which leads to a better resolution image.

The proposed target classification method is presented in Fig. 4.12. First, we extract the area that contains the objects by thresholding the migrated image of each waveform. Then, we apply boundary detection algorithm to separate the region of each target. For every pixel inside target region, we calculate the amplitude ratios of $1^{\text {st }}, 2^{\text {nd }}$, and $3^{\text {rd }}$ order HP waveforms with respect to $0^{\text {th }}$ order HP waveform. We use the combinations of these ratios as the features and pass them to SVM classifier. Then, we accumulate the result over the region of the corresponding target and determine the material based on the majority classes. We consider only a simple classification case with 2 classes: low dielectric constant material for class 1 and high dielectric constant material or conductor for class 2. 


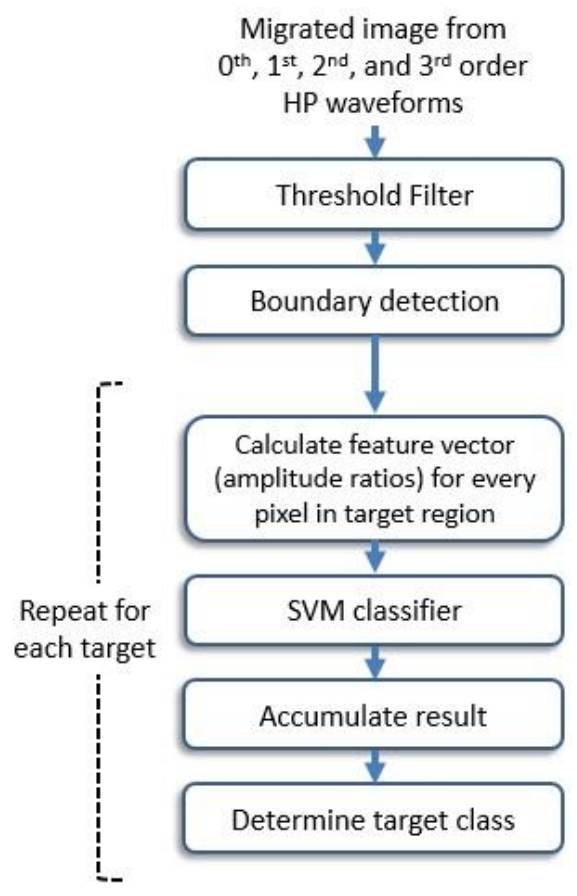

Fig. 4.12: Diagram of the classification method.

To obtain the training data, we select 5 scenarios from simulations in Fig. 4.10. The first and the second scenarios are taken from horizontal plate target $\left(\theta=0^{\circ}\right)$ in Fig. 4.10(b) with low dielectric material and PEC material respectively. For the third and the fourth scenarios, we use cylinder target presented in Fig. 4.10(a) with diameter of $3 \mathrm{~cm}$. The cylinder is made of low dielectric material in the third scheme and high dielectric material in the fourth scheme. Finally, we use the PEC cylinder with diameter of $1 \mathrm{~cm}$ as the last scenario. These objects can be easily found in practice.

Since we have 3 features from the amplitude ratios of the waveforms, we investigate all possible combinations of these features. Thus, we have 4 types of feature vectors. We extract these features from the aforementioned scenarios and train the SVM classifier. In order to test the resulting parameters, we apply the remaining data from Fig. 4.10 that are not used in the training process, and then calculate the percentage of the classification error. The results indicate acceptable accuracy as provided in Table 4.2.

Table 4.2: Percentage of ClassificAtion ERrors

\begin{tabular}{|l|c|}
\hline \multicolumn{1}{|c|}{ Feature vectors } & Percentage of Error \\
\hline Amplitude ratios of $1^{\text {st }}$ and $2^{\text {nd }}$ order HP & $7.2 \%$ \\
\hline Amplitude ratios of $2^{\text {nd }}$ and $3^{\text {rd }}$ order HP & $9.9 \%$ \\
\hline
\end{tabular}




\begin{tabular}{|l|c|}
\hline \multicolumn{1}{|c|}{ Feature vectors } & Percentage of Error \\
\hline Amplitude ratios of $1^{\text {st }}$ and $3^{\text {rd }}$ order HP & $10.1 \%$ \\
\hline Amplitude ratios of $1^{\text {st }}, 2^{\text {nd }}$, and $3^{\text {rd }}$ order HP & $9.1 \%$ \\
\hline
\end{tabular}

\subsection{Performance Analysis on Several Target Shapes and Materials}

To assess the performance of the proposed waveform diversity method, we arrange several simulations with various target shapes and materials. We divide the evaluation into 2 parts. In the first part, we investigate the imaging performance of the combination method proposed in Subsection 4.2.1 in terms of resolution and side-lobe level. In the second part, we test the classification technique proposed in Subsection 4.2.2 on distributed targets with various materials.

\subsubsection{Analysis on Combination Method}

To observe the resolution and the side-lobe level of each waveform, we repeat the simulation on point target given in Fig. 3.11. As described in Section 4.2, we use a single pair of transmitter and receiver with sampling distance of $1.5 \mathrm{~cm}$ and collect the data by using each waveform independently. Then, we apply the proposed filtering method to combine the data of these waveforms. Finally, we perform f-k migration to all the resulting data and extract their beam-patterns. The results are given in Fig. 4.13 for horizontal beam-patterns and Fig. 4.14 for vertical beam-patterns. 


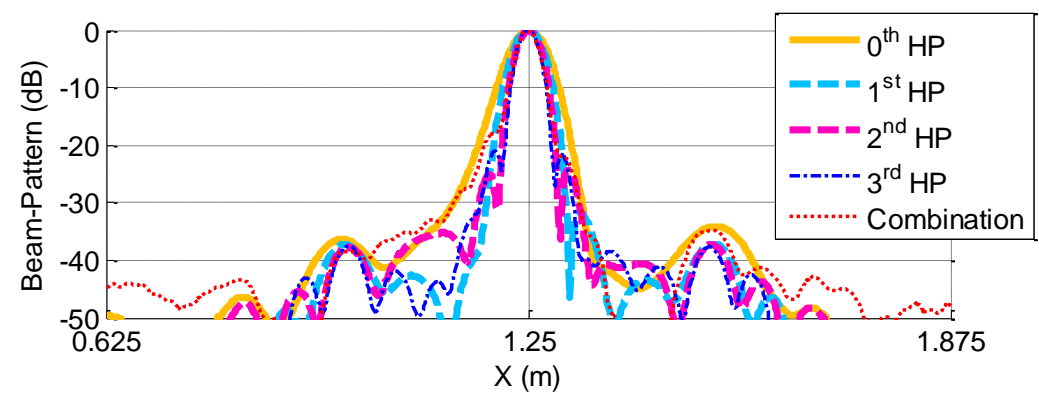

(a) Horizontal beam-patterns

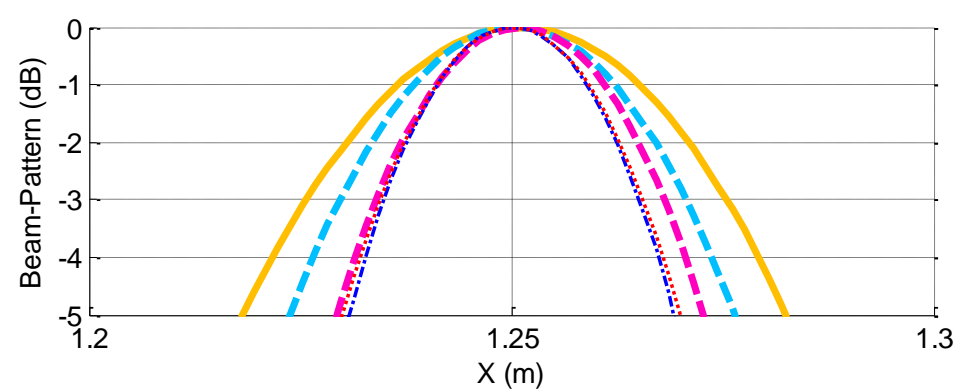

(b) Zoom-in of (a)

Fig. 4.13: Horizontal beam-patterns of HP waveforms and their combination.

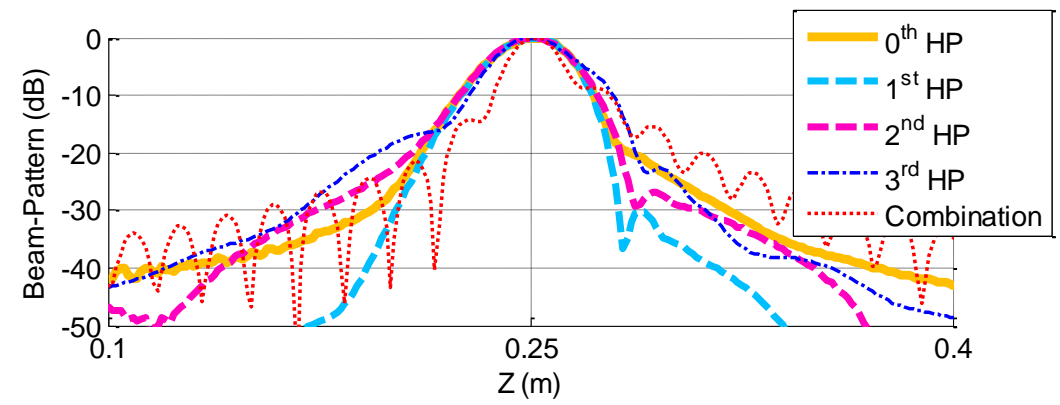

(a) Vertical beam-patterns

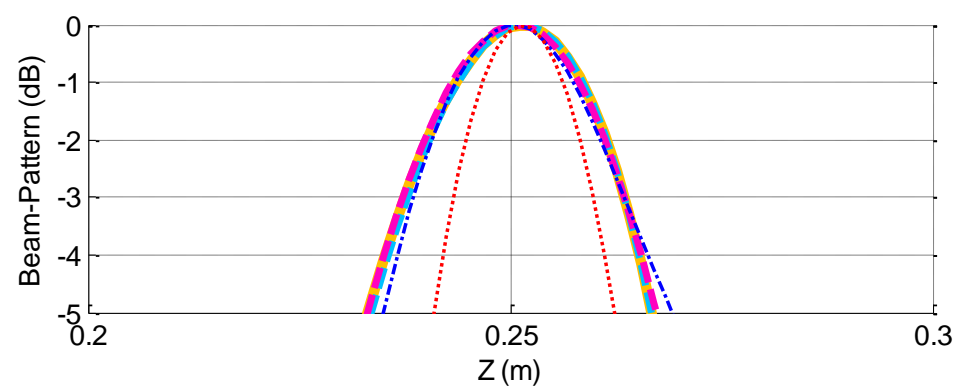

(b) Zoom-in of (a)

Fig. 4.14: Vertical beam-patterns of HP waveforms and their combination.

As we can see, among all the waveforms, $0^{\text {th }}$ order HP exhibits the lowest horizontal resolution while $3^{\text {rd }}$ order HP offers the highest horizontal resolution. On the other hand, the proposed combination method successfully achieves almost similar horizontal resolution to the $3^{\text {rd }}$ order HP and better vertical resolution compared to all the waveforms. To confirm 
these properties, we calculate the corresponding 3-dB resolutions as given in Table 4.3. These results are in agreement with our direct observation on the beam-patterns.

Table 4.3: RESOlution OF EACH WAVEFORM AND THE COMBINATON METHOD

\begin{tabular}{|l|c|c|}
\hline Waveform & Horizontal Resolution $(\mathbf{c m})$ & Vertical Resolution $(\mathbf{c m})$ \\
\hline $0^{\text {th }}$ order HP & 5.0 & 2.8 \\
\hline $1^{\text {st }}$ order HP & 4.3 & 2.8 \\
\hline $2^{\text {nd }}$ order HP & 3.6 & 2.8 \\
\hline $3^{\text {rd }}$ order HP & 3.1 & 2.6 \\
\hline Combination method & 3.1 & 1.9 \\
\hline
\end{tabular}

We further verify the advantages of the proposed method by conducting simulation on distributed targets as shown in Fig. 4.15. The scenario contains six cylinders with diameter of $3 \mathrm{~cm}$ that are aligned horizontally and a tilted plate with width of $30 \mathrm{~cm}$ and thickness of 1 $\mathrm{cm}$. The distances between each successive cylinder increase from $7 \mathrm{~cm}$ to $11 \mathrm{~cm}$. All these targets are made of PEC, while the subsurface is made of concrete material with relative permittivity 6 , relative permeability 1 , and conductivity $0.001 \mathrm{~S} / \mathrm{m}$.

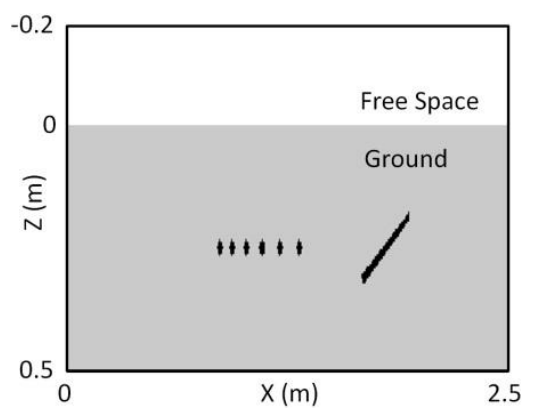

Fig. 4.15: Target configuration.

The imaging results are provided in Fig. 4.16. As we can see, the $0^{\text {th }}$ order HP is unable to resolve the cylinders due to its low resolution. By contrast, the $3^{\text {rd }}$ order HP offers the best imaging result over these cylinders. However, the plate appears to be distorted by the $3^{\text {rd }}$ order HP, while the $0^{\text {th }}$ order HP exhibits the clearest image on such target. The proposed combination method in Fig. 4.16(e) successfully resolves the closely located cylinders and the side of the plate. These results confirm the advantages of the proposed method that are predicted theoretically in Subsection 4.2.1. 


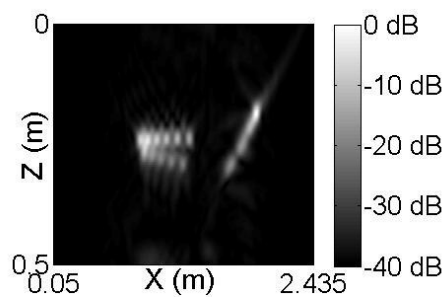

(a) $0^{\text {th }}$ order HP

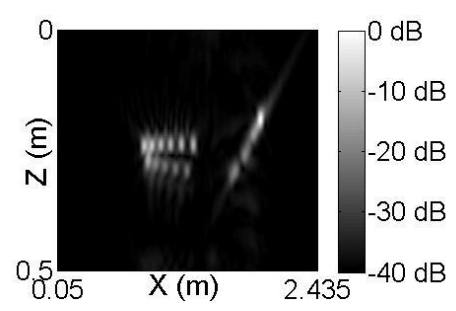

(b) $1^{\text {st }}$ order HP

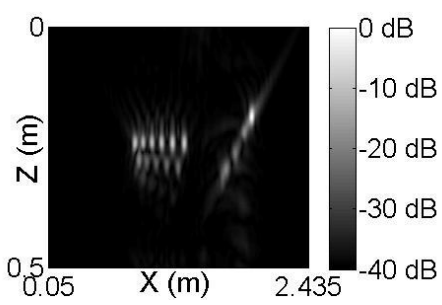

(c) $2^{\text {nd }}$ order HP

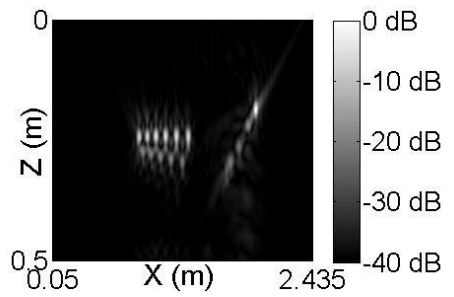

(d) $3^{\text {rd }}$ order HP

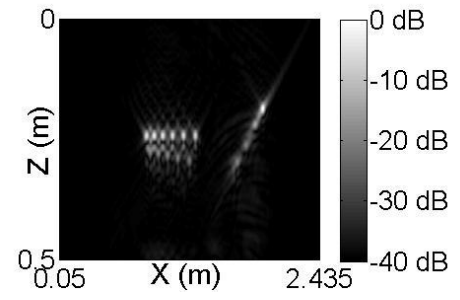

(e) Combination method

Fig. 4.16: Simulation results.

\subsubsection{Analysis on Target Classification Method}

In this subsection, we analyze the target classification method proposed in Subsection 4.2.2. We set a simulation with distributed targets as shown in Fig. 4.17. Such scenario can be encountered in the inspection of rebars inside concrete structure. In this simulation, the rebars are modelled as PEC cylinders with diameter $2.5 \mathrm{~cm}$ and spacing $20 \mathrm{~cm}$. There are 2 defects located above the rebars. The first one on the left is a water-filled crack with width of $10 \mathrm{~cm}$ and thickness of $1 \mathrm{~cm}$. Meanwhile, the second defect on the right is a void crack with width of $40 \mathrm{~cm}$ and thickness of $2 \mathrm{~cm}$. These cracks are generated by using fractal model with dimension of 2 and range of $5 \mathrm{~cm}$. The subsurface comprises 2 layers. The upper layer is a homogeneous concrete with relative permittivity 6 , relative permeability 1 , and conductivity $0.001 \mathrm{~S} / \mathrm{m}$. Meanwhile, the second layer is a granular material with relative permittivity 11.1, relative permeability 1 , and conductivity $0.005 \mathrm{~S} / \mathrm{m}$.

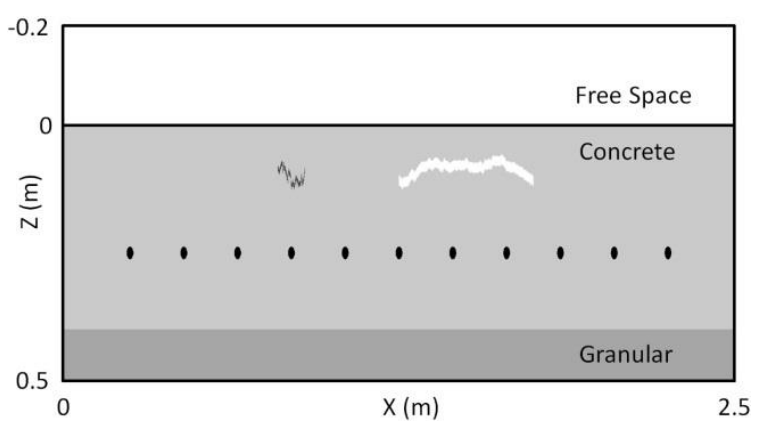

Fig. 4.17: Target configuration. 
The images of the individual waveforms are given in Fig. 4.18, while the target classification results are shown in Fig. 4.19. By comparing the two images, one can see that the proposed method provides further information regarding the target. In addition, by referring to the real scenario, we can observe that all combinations of the features demonstrate approximately correct classification results. The void crack is classified into the first class, while the rebars are classified into the second class. Although some parts of the water-filled crack belong to the first class, we can see that overall the reflection of this target are mainly classified into the second class. We can also observe that the granular layer is undetected by the proposed method due to its low reflection compared to the other targets.

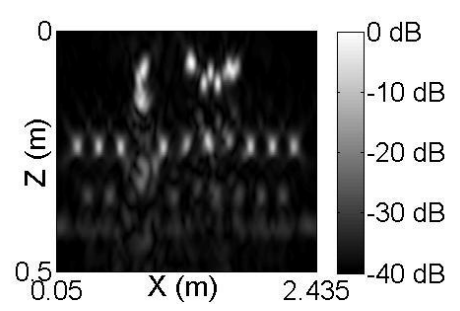

(a) $0^{\text {th }}$ order HP

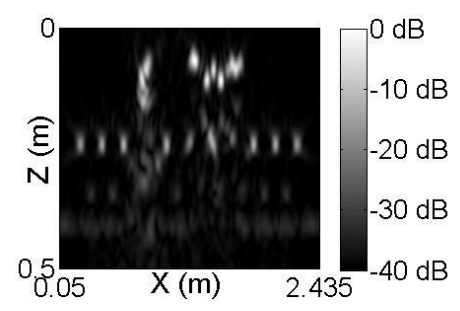

(b) $1^{\text {st }}$ order HP

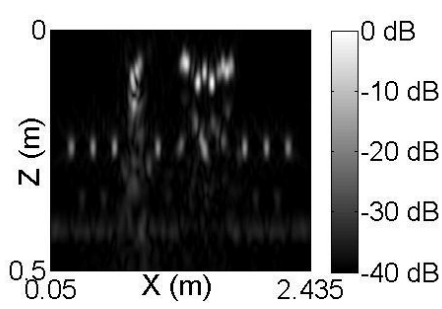

(c) $2^{\text {nd }}$ order HP

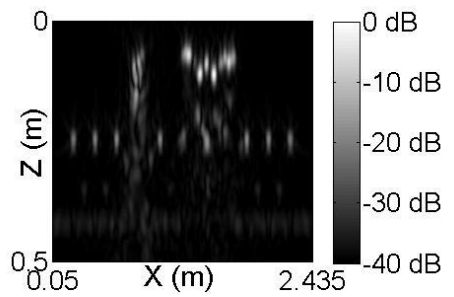

(d) $3^{\text {rd }}$ order HP

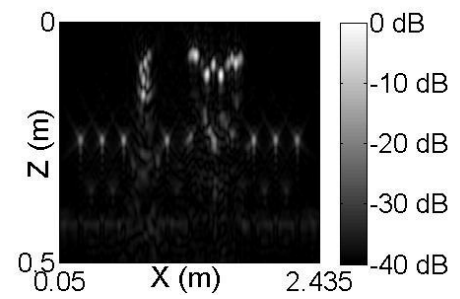

(e) Combination method

Fig. 4.18: Simulation results. 


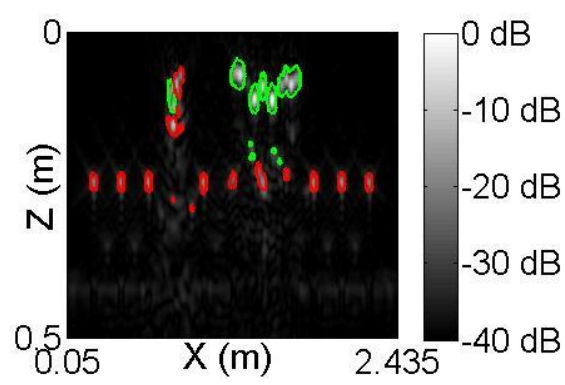

(a) Features: $1^{\text {st }}$ and $2^{\text {nd }}$ order HP

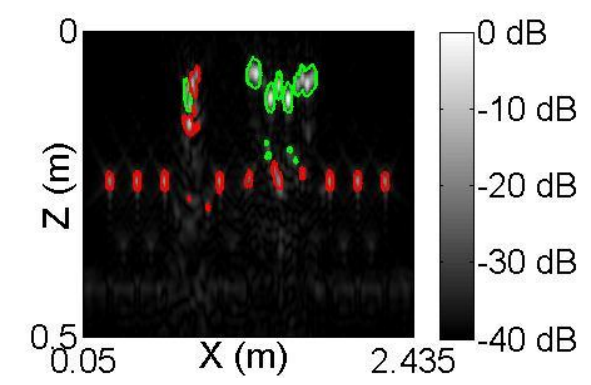

(c) Features: $1^{\text {st }}$ and $3^{\text {rd }}$ order HP

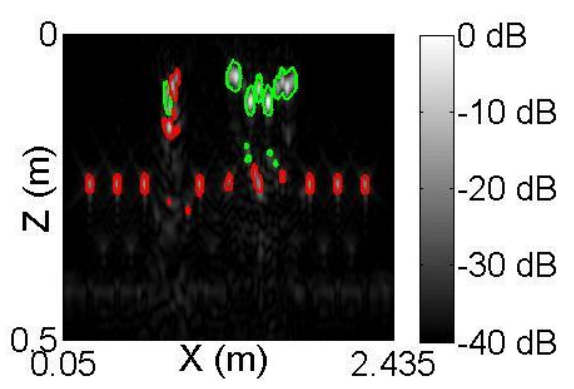

(b) Features: $2^{\text {nd }}$ and $3^{\text {rd }}$ order HP

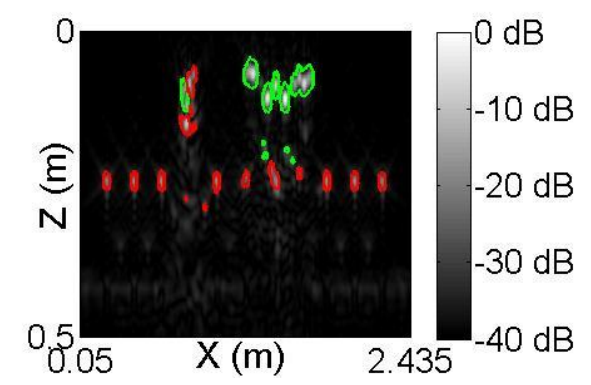

(d) Features: $1^{\text {st }}, 2^{\text {nd }}$, and $3^{\text {rd }}$ order HP

Fig. 4.19: Target classification results with green line for class 1 and red line for class 2.

\subsection{Concluding Remarks}

In this chapter, we have presented the implementation of the proposed waveform diversity for UWB GPR. Two processing approaches are proposed. In the first method, the data from all waveforms are combined by using a particular filtering technique. Meanwhile, in the second approach, a binary classification method based on target material is formulated. Compared to the existing classification methods, the proposed technique extracts the features from the migrated images and utilizes simplified training scenarios which are more feasible to conduct in practice.

We have shown theoretically that the combination method can improve both the horizontal and the vertical resolutions. These results are further verified by simulation data. The target classification method is also evaluated on simulation data, and the results demonstrate satisfactory accuracy. In the proposed method, the targets are simply classified into 2 classes based on the amplitude ratios between the waveforms. This technique may serve as a preliminary concept to more complex approaches by using other features from waveform diversity. 


\section{CHAPTER 5}

\section{The Polarization Diversity for UWB GPR Systems}

The last diversity aspect considered in this study is polarization. As discussed earlier in Chapter 2, a number of works have been addressed to the development of polarimetric GPR systems, which are mainly inspired by polarimetric synthetic aperture radar in remote sensing field. In this chapter, we present further investigation of polarization diversity for UWB GPR systems. Polarimetric synthetic aperture radar concepts are first introduced in Section 5.1, along with detailed descriptions of polarimetric decomposition techniques. Then, these concepts for UWB GPR systems are formulated in Section 5.2. The evaluation of their performance is conducted based on simulation and experimental data, which are provided in Section 5.3 and 5.4 respectively. In Section 5.5, the effects of sensor configurations on the resulting polarimetric characteristics are analyzed. Finally, all the results are summarized in the Section 5.5.

\subsection{Review of Existing Theory}

\subsubsection{Polarimetric in Synthetic Aperture Radar System}

Synthetic aperture radar (SAR) is a microwave remote sensing method that utilizes the movement path of the platform to form a synthetic long aperture antenna and achieve high resolution image. This technology has been developed over decades as a tool to map the earth surface with high spatial resolution and imaging capability in day, night, and through almost all weather conditions. Typically, SAR system is implemented on airborne or space-borne systems with a particular operating frequency, i.e., P-band, L-band, C-band, and X-band. Nowadays, this system is available in variant measurement techniques such as strip-map, spot-light, interferometry, and polarimetric. The latter one, which is well-known as polarimetric synthetic aperture radar (PolSAR), is the main focus of this section.

In the early development of polarimetric method on imaging radar, polarimetric information was used for characterization of aircraft targets. Nowadays, this technique has been extended into various purposes. One of the most remarkable applications of PolSAR is terrain and land-use classification [117]-[121]. Another important application of PolSAR is forest mapping and classification, in which polarimetric technique is combined with interferometry 
method for estimation of biomass and forest heights [122], [123]. Furthermore, this technique has been developed for assessment and monitoring natural phenomena such as deforestation, ocean, and hazard [124]-[126]. Besides the observation on natural scenes, polarimetric technique has also been used to analyze the man-made structures such as bridge construction [127].

The first fully-polarimetric system was implemented in 1985 on airborne system AIRSAR developed under NASA Jet Propulsion Laboratory (JPL). In 1994, the space-borne polarimetric system began to be deployed on shuttle imaging radar-C (SIR-C) and X-band synthetic aperture radar (X-SAR), which were the collaboration of space shuttle research between NASA, German Space Agency (DARA), and Italian Space Agency (ASI). The first civilian satellite that utilized dual-polarization measurement was ENVISAT, which was launched in 2002 under European Space Agency (ESA). Japanese Space Agency (JAXA) launched the first fully-polarimetric satellite ALOS in 2006. Afterward, a number of polarimetric satellite systems were developed and launched by various research institutions.

Based on the available polarimetric measurements, there are 2 types of PolSAR system: fullypolarimetric and compact-polarimetric. In fully-polarimetric SAR system, the data acquisition is conducted in combination of 2 orthogonal polarization bases on both transmitter and receiver. The common practice is by using linear polarization in direction of horizontal (H) and vertical (V) axes. Such configuration produces 4 datasets denoted by $\mathrm{HH}$, $\mathrm{HV}, \mathrm{VH}$, and $\mathrm{VV}$, where the first term represents the transmitter polarization and the second term represents the receiver polarization. By contrast, the compact-polarimetric system employs only 1 polarization basis on its transmitter, while the receiver collects the data in 2 orthogonal polarization bases. This technique was developed to compromise the constraints in complexity, bandwidth, and range swath coverage.

In this study, the fully-polarimetric system will be considered. We provide several formulations and signal processing concepts of the fully-polarimetric PolSAR system in the following subsections.

\subsubsection{Polarimetric Measurement and Parameter}

Based on Maxwell's equation, electromagnetic wave can be described in terms of electric field and magnetic field components. As vector field, these electric and magnetic field components have both magnitude and direction. Polarization is defined as the resultant time 
locus of the electric field $\overrightarrow{\mathbf{E}}$ in the plane perpendicular to the direction of propagation of the wave. The electric field of a polarized wave can be formulated in a 2-dimensional complex space column vector as given in (5.1), where $e_{x}$ is the $x$-polarized component, $e_{y}$ is the $y$ polarized component, $E_{0 x}$ is the magnitude of $e_{x}, \delta_{x}$ is the phase of $e_{x}, E_{0 y}$ is the magnitude of $e_{y}$, and $\delta_{y}$ is the phase of $e_{y}$. This representation of electric field $\overrightarrow{\mathbf{E}}$ is well-known as Jones vector.

$$
\overrightarrow{\mathbf{E}}=\left[\begin{array}{l}
e_{x} \\
e_{y}
\end{array}\right]=\left[\begin{array}{l}
E_{0 x} e^{j \delta_{x}} \\
E_{0 y} e^{j \delta_{y}}
\end{array}\right]
$$

The electric field in (5.1) is expressed in $\mathrm{X}$ and $\mathrm{Y}$ linear polarization bases. In general, Jones vector can be expressed in any 2 orthogonal polarization bases. Furthermore, we can transform the representation of Jones vector from one bases to another bases by using unitary transformation matrix $\boldsymbol{U}_{2}(\phi, \tau, \alpha)$ as given in (5.2). In (5.2), the unitary transform matrix can be further parameterized in terms of $\alpha, \tau$, and $\phi$, which are related to the polarization ellipse. Although it is possible to use another orthogonal polarization bases, for simplicity all the equations provided in this chapter will be represented in $\mathrm{X}$ and $\mathrm{Y}$ linear polarization bases.

$$
\overrightarrow{\mathbf{E}}^{\prime}=\mathbf{U}_{2}(\phi, \tau, \alpha) \overrightarrow{\mathbf{E}}=\left[\begin{array}{cc}
\cos \phi & -\sin \phi \\
\sin \phi & \cos \phi
\end{array}\right]\left[\begin{array}{cc}
\cos \tau & j \sin \tau \\
j \sin \tau & \cos \tau
\end{array}\right]\left[\begin{array}{cc}
e^{j \alpha} & 0 \\
0 & e^{-j \alpha}
\end{array}\right] \overrightarrow{\mathbf{E}}
$$

A target in the scattering process acts as polarization transformer, by which the polarization of the incident wave is converted into another polarization state. Fig. 5.1 illustrates the scattering process, where the incident wave polarization plane is given by $(x, y)$ and the scattered wave polarization plane is given by $\left(x^{\prime}, y^{\prime}\right)$. The polarization transformation can be formulated by the so-called Sinclair matrix $S$ as given in (5.3), where $\overrightarrow{\mathbf{E}}_{\mathbf{I}}$ is the vector of the incident wave, $\overrightarrow{\mathbf{E}}_{\mathrm{S}}$ is the vector of the scattered wave, and $R$ is the distance from the target to the observation point. Similar to the Jones vector case, Sinclair matrix can be transformed into another bases by using unitary transformation matrix $\boldsymbol{U}_{2}(\phi, \tau, \alpha)$ as given in (5.4). 


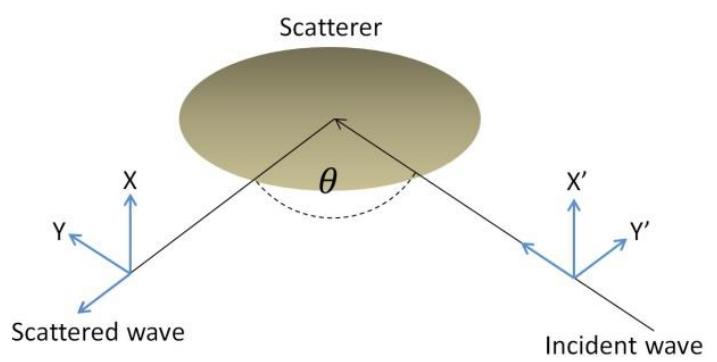

Fig. 5.1: Coordinate of scattering process.

$$
\begin{gathered}
\overrightarrow{\mathbf{E}}_{\mathbf{S}}=\frac{e^{-j k R}}{R} \mathbf{S} \overrightarrow{\mathbf{E}}_{\mathbf{I}}=\frac{e^{-j k R}}{R}\left[\begin{array}{cc}
S_{X X} & S_{X Y} \\
S_{Y X} & S_{Y Y}
\end{array}\right] \overrightarrow{\mathbf{E}}_{\mathbf{I}} \\
\mathbf{S}^{\prime}=\mathbf{U}_{\mathbf{2}}(\phi, \tau, \alpha) \mathbf{S U}_{\mathbf{2}}(\phi, \tau, \alpha)^{H}
\end{gathered}
$$

Based on the angle between the incident wave and the scattered wave $(\theta)$, there are 3 special configurations in scattering process: forward scattering $\left(\theta=180^{\circ}\right)$, lateral scattering $\left(\theta=90^{\circ}\right)$, and backscattering $\left(\theta=0^{\circ}\right)$. PolSAR signal processing mainly considers the last case, i.e., the backscattering configuration. Furthermore, we assume the monostatic configuration, in which the transmitter and the receiver are collocated, and the cross-polarized components $S_{X Y}$ and $S_{Y X}$ are equal.

In SAR signal processing, an image is constructed by coherently processing returns from successive pulses, which in turn causes variation in pixel intensity. Such variation is so-called as speckle and appears as a granular pattern in the image. Speckle becomes an important issue since it degrades the image quality and feature classification. In order to reduce the speckle effect, some polarimetric processing employ second order statistic parameters instead of Sinclair matrix. Two most common parameters are coherency matrix $(\boldsymbol{T})$ and covariance matrix $(\boldsymbol{C})$, which are extracted by averaging process over a particular window in the image. The coherency matrix is given in (5.5), where $\boldsymbol{k}$ is the vector form of Sinclair matrix in Pauli basis as given in (5.6). Meanwhile, the covariance matrix is given in (5.7), where $\boldsymbol{\Omega}$ is the vector form of Sinclair matrix in lexicographic basis as given in (5.8).

$$
\begin{aligned}
& \mathbf{T}=\left\langle\mathbf{k k}^{H}\right\rangle \\
& \mathbf{k}=\frac{1}{\sqrt{2}}\left[S_{X X}+S_{Y Y} \quad S_{X X}-S_{Y Y} \quad 2 S_{X Y}\right]^{T} \\
& \mathbf{C}=\left\langle\boldsymbol{\Omega} \boldsymbol{\Omega}^{H}\right\rangle \\
& \boldsymbol{\Omega}=\left[\begin{array}{lll}
S_{X X} & \sqrt{2} S_{X Y} & S_{Y Y}
\end{array}\right]^{T}
\end{aligned}
$$


In (5.5) to (5.8), the covariance matrix and coherency matrix are expressed in the $\mathrm{X}$ and $\mathrm{Y}$ linear polarization bases. These matrices can be transformed into another polarization bases by applying unitary transformation matrix $\boldsymbol{U}_{3}(\phi, \tau, \alpha)$ for coherency matrix as given in (5.9) and $\boldsymbol{U}_{\mathbf{3}}(\rho, \xi)$ for covariance matrix as given in (5.10). Furthermore, $\boldsymbol{U}_{\mathbf{3}}(\phi, \tau, \alpha)$ and $\boldsymbol{U}_{\mathbf{3}}(\rho, \xi)$ can be parameterized in terms of $(\alpha, \tau, \phi)$ and $(\rho, \xi)$ as shown in (5.11) and (5.12) respectively. Notice that the parameters $(\alpha, \tau, \phi)$ are linked to the associated parameters in transformation matrix $\boldsymbol{U}_{2}(\phi, \tau, \alpha)$ for Jones vector and Sinclair matrix.

$$
\begin{aligned}
& \mathbf{T}^{\mathbf{\prime}}=\mathbf{U}_{3}(\phi, \tau, \alpha) \mathbf{T} \mathbf{U}_{3}(\phi, \tau, \alpha)^{H} \\
& \mathbf{C}^{\prime}=\mathbf{U}_{\mathbf{2}}(\rho, \xi) \mathbf{C} \mathbf{U}_{\mathbf{2}}(\rho, \xi)^{H} \\
& \mathbf{U}_{3}(\phi, \tau, \alpha)=\left[\begin{array}{ccc}
1 & 0 & 0 \\
0 & \cos 2 \phi & \sin 2 \phi \\
0 & -\sin 2 \phi & \cos 2 \phi
\end{array}\right]\left[\begin{array}{ccc}
\cos 2 \tau & 0 & j \sin 2 \tau \\
0 & 1 & 0 \\
j \sin 2 \tau & 0 & \cos 2 \tau
\end{array}\right]\left[\begin{array}{ccc}
\cos 2 \alpha & j \sin 2 \alpha & 0 \\
j \sin 2 \alpha & \cos 2 \alpha & 0 \\
0 & 0 & 1
\end{array}\right] \\
& \mathbf{U}_{3}(\rho, \xi)=\frac{1}{1+|\rho|^{2}}\left[\begin{array}{ccc}
e^{2 j \xi} & \sqrt{2} \rho e^{2 j \xi} & \rho^{2} e^{2 j \xi} \\
-\sqrt{2} \rho^{*} & 1-|\rho|^{2} & \sqrt{2} \rho \\
\rho^{* 2} e^{-2 j \xi} & -\sqrt{2} \rho^{*} e^{-2 j \xi} & e^{-2 j \xi}
\end{array}\right]
\end{aligned}
$$

Theoretically, a pure single target produces a particular Sinclair matrix. However, practically the scattered wave comes from combination of target returns and unwanted components, which obscure the polarimetric characteristic of the desired target. Moreover, coherency and covariance matrices involve averaging process over certain window in the image, which distort the polarimetric signature of the individual targets within such window. The main objective of polarimetric decomposition techniques is to provide target interpretation on those polarimetric parameters based on particular assumptions. These methods can be classified into 2 main categories: coherent decomposition and incoherent decomposition. In the former one, the decomposition is performed on Sinclair matrix, while in the latter one the decomposition is applied to the second order statistic parameters, i.e., coherency matrix and covariance matrix. We present detailed description of these decomposition approaches in the following subsections. In addition, we also include the discussion on $\mathrm{H}$-alpha decomposition, which is an unsupervised classification method based on entropy and alpha angle parameters. 


\subsubsection{Polarimetric Coherent Decomposition}

Coherent decomposition interprets the Sinclair matrix as combinations of several basic scattering mechanisms. Due to this straightforward utilization of Sinclair matrix, coherent decomposition leads to a lower computational complexity compared to incoherent decomposition at the expense of noisier image. In this study, we explore two decomposition approaches that belong to this class, namely Pauli decomposition and Krogager decomposition.

In Pauli decomposition [128], the Sinclair matrix is expressed as a sum of Pauli matrices as given in (5.13). Each Pauli component represents a particular scattering mechanism. The first component $(a)$ is a single or odd-bounce scattering, the second component $(b)$ is a diplane scattering with orientation of $0^{\circ}$, while the last component $(c)$ is a diplane scattering with orientation of $45^{\circ}$.

$$
\mathbf{S}=\frac{a}{\sqrt{2}}\left[\begin{array}{ll}
1 & 0 \\
0 & 1
\end{array}\right]+\frac{b}{\sqrt{2}}\left[\begin{array}{cc}
1 & 0 \\
0 & -1
\end{array}\right]+\frac{c}{\sqrt{2}}\left[\begin{array}{ll}
0 & 1 \\
1 & 0
\end{array}\right]
$$

Solving each term in (5.13), we can obtain

$$
\begin{aligned}
& a=\left(S_{X X}+S_{Y Y}\right) / \sqrt{2} \\
& b=\left(S_{X X}-S_{Y Y}\right) / \sqrt{2} \\
& c=\sqrt{2} S_{X Y}
\end{aligned}
$$

In Krogager decomposition [129], [130], the Sinclair matrix is decomposed into 3 components that represent scattering from sphere $\left(k_{S}\right)$, diplane $\left(k_{D}\right)$, and helix $\left(k_{H}\right)$ under transformation of rotation angle $\theta$ as formulated in (5.15).

$$
\mathbf{S}_{(\mathbf{X}, \mathbf{Y})}=e^{j \phi}\left\{e^{j \phi_{S}} k_{S}\left[\begin{array}{ll}
1 & 0 \\
0 & 1
\end{array}\right]+k_{D}\left[\begin{array}{cc}
\cos 2 \theta & \sin 2 \theta \\
\sin 2 \theta & -\cos 2 \theta
\end{array}\right]+k_{H} e^{\mp j 2 \theta}\left[\begin{array}{cc}
1 & \pm j \\
\pm j & -1
\end{array}\right]\right\}
$$

To solve (5.15), we can first convert the linear $X Y$ polarization bases of $S$ into right-left circular bases by using the transformation formula given in (5.16).

$$
\mathbf{S}_{(\mathbf{R}, \mathbf{L})}=\left[\begin{array}{ll}
S_{R R} & S_{R L} \\
S_{R L} & S_{L L}
\end{array}\right]=e^{j \phi}\left\{e^{j \phi_{S}} k_{S}\left[\begin{array}{ll}
0 & j \\
j & 0
\end{array}\right]+k_{D}\left[\begin{array}{cc}
e^{j 2 \theta} & 0 \\
0 & -e^{-j 2 \theta}
\end{array}\right]+k_{H}\left[\begin{array}{cc}
e^{j 2 \theta} & 0 \\
0 & 0
\end{array}\right]\right\}
$$

Thus, the Sinclair matrix in circular polarization basis can be explicitly expressed as 


$$
\begin{aligned}
& S_{L L}=S_{H H}-S_{V V}+2 i S_{H V} \\
& S_{R L}=i\left(S_{H H}+S_{V V}\right) \\
& S_{R R}=S_{V V}-S_{H H}+2 i S_{H V}
\end{aligned}
$$

Finally, we can solve (5.17) and obtain the Krogager components as shown in (5.18).

$$
\begin{aligned}
& k_{S}=\left|S_{R L}\right| \\
& k_{D}=\min \left(\left|S_{R R}\right|,\left|S_{L L}\right|\right) \\
& k_{H}=|| S_{R R}|-| S_{L L}||
\end{aligned}
$$

In PolSAR, the results of coherent decomposition are commonly visualized as an RGB image. In Pauli decomposition, the color-codes are red $=|b|^{2}$, green $=|c|^{2}$, blue $=|a|^{2}$, while in Krogager decomposition we have red $=k_{D}$, green $=k_{H}$, and blue $=k_{S}$.

\subsubsection{Polarimetric incoherent decomposition}

By contrast to the coherent decomposition, the incoherent decomposition provides interpretation on coherency and covariance matrices. Due to the utilization of second order statistics, this technique is more robust against noise and clutter components compared to coherent decomposition. Based on the physical assumption made on the scattering mechanism, we can classify incoherent polarimetric decomposition into 3 types: decomposition based on dichotomy of Kennaugh matrix, decomposition based on eigenvector and eigenvalue, and model-based decomposition.

\section{Decomposition Based on Dichotomy of Kennaugh Matrix}

In this approach, the data is considered as a single target with some residual components. Thus, the coherency matrix $(\boldsymbol{T})$ can be decomposed into the coherency matrix of the target $\left(\boldsymbol{T}_{\boldsymbol{0}}\right)$ and the coherency matrix of the clutter $\left(\boldsymbol{T}_{N}\right)$. To differentiate the target from the clutter component, $\boldsymbol{T}_{\boldsymbol{0}}$ is assumed to be a rank-1 matrix, while $\boldsymbol{T}_{\boldsymbol{N}}$ is assumed to have roll-invariant properties. For our study, we analyze only two decomposition methods in this approach, i.e., Huynen decomposition [131] and Barnes-Holm decomposition [132].

In Huynen decomposition, the coherency matrix can be formulated in terms of Huynen parameters as presented in (5.19). 


$$
\mathbf{T}=\left[\begin{array}{ccc}
2\left\langle A_{0}\right\rangle & \langle C\rangle-j\langle D\rangle & \langle H\rangle+j\langle G\rangle \\
\langle C\rangle+j\langle D\rangle & \left\langle B_{0}\right\rangle+\langle B\rangle & \langle E\rangle+j\langle F\rangle \\
\langle H\rangle-j\langle G\rangle & \langle E\rangle-j\langle F\rangle & \left\langle B_{0}\right\rangle-\langle B\rangle
\end{array}\right]
$$

Since the clutter part has roll-invariant behavior, the components $B_{0}, B, E$, and $F$ can be decomposed into target components $\left(B_{0 T}, B_{T}, E_{T}, F_{T}\right)$ and clutter components $\left(B_{0 N}, B_{N}, E_{N}, F_{N}\right)$ as given in (5.20).

$$
\begin{aligned}
B_{0} & =B_{0 T}+B_{0 N} \\
B & =B_{T}+B_{N} \\
E & =E_{T}+E_{N} \\
F & =F_{T}+F_{N}
\end{aligned}
$$

On the other hand, the coherency matrix of the target must be a rank 1 matrix, so the target components must satisfy (5.21).

$$
\begin{aligned}
2 A_{0}\left(B_{0 T}+B_{T}\right) & =C^{2}+D^{2} \\
2 A_{0}\left(B_{0 T}-B_{T}\right) & =G^{2}+D^{2} \\
2 A_{0} E_{T} & =C H-D G \\
2 A_{0} F_{T} & =C G+D H
\end{aligned}
$$

We can obtain the corresponding target components by solving (5.21). Finally, the coherency matrix of the target can be written as

$$
\mathbf{T}_{\mathbf{0}}=\left[\begin{array}{ccc}
2\left\langle A_{0}\right\rangle & \langle C\rangle-j\langle D\rangle & \langle H\rangle+j\langle G\rangle \\
\langle C\rangle+j\langle D\rangle & B_{0 T}+B_{T} & E_{T}+j F_{T} \\
\langle H\rangle-j\langle G\rangle & E_{T}-j F_{T} & B_{0 T}-B_{T}
\end{array}\right]
$$

Barnes-Holm decomposition adopts a more general approach than Huynen decomposition. Based on roll-invariant properties, the null-space of $\boldsymbol{T}_{N}$ must not change under rotational transform. Thus, the eigenvectors of rotational transform matrix $\boldsymbol{U}_{\mathbf{3}}(\theta)$ must be the null-space of $\boldsymbol{T}_{N}$ as expressed in (5.23), where $i=1,2,3$, and $q_{i}$ is the eigenvectors of $\boldsymbol{U}_{3}(\theta)$ given by (5.24).

$$
\begin{aligned}
& \mathbf{U}_{3}(\theta) \mathbf{q}_{\mathbf{i}}=\lambda \mathbf{q}_{\mathbf{i}} \\
& \mathbf{T}_{\mathbf{N}} \mathbf{q}_{\mathbf{i}}=0
\end{aligned}
$$




$$
\begin{aligned}
& \mathbf{q}_{1}=\left[\begin{array}{lll}
1 & 0 & 0
\end{array}\right]^{T} \\
& \mathbf{q}_{2}=\left[\begin{array}{lll}
0 & 1 & j
\end{array}\right]^{T} / \sqrt{2} \\
& \mathbf{q}_{3}=\left[\begin{array}{lll}
0 & j & 1
\end{array}\right]^{T} / \sqrt{2}
\end{aligned}
$$

It can be shown that the first eigenvector $\boldsymbol{q}_{1}$ leads to Huynen decomposition. Barnes-Holm decomposition utilizes the second and third eigenvectors. In such decomposition, the coherency matrix of the target can be obtained as

$$
\mathbf{T}_{\mathbf{0} \mathbf{i}}=\mathbf{k}_{\mathbf{0} \mathbf{i}} \mathbf{k}_{\mathbf{0 i}}{ }^{H} \quad \text { with } i=2,3
$$

where $\boldsymbol{k}_{\boldsymbol{\theta} \boldsymbol{i}}$ is given by

$$
k_{0 i}=\frac{T_{q_{i}}}{\sqrt{\mathbf{q}_{i}^{H} \mathbf{T} \mathbf{q}_{i}}}
$$

Similar to coherent decomposition, the decomposition results in this group can be presented in RGB image. The color-codes in this case are red $=T_{22 T}$, green $=T_{33 T}$, and blue $=T_{11 T}$, where $T_{i j T}$ is the $(i, j)$-th element of target coherency matrix $\boldsymbol{T}_{\boldsymbol{0}}$.

\section{Decomposition Based on Eigen-Vector and Eigen-Value}

This approach suggests the decomposition of coherency and covariance matrices into several independent scattering mechanisms based on eigen-value decomposition as expressed in (5.27) and (5.28).

$$
\begin{gathered}
\mathbf{T}=\mathbf{U} \boldsymbol{\Sigma} \mathbf{U}^{-1}=\sum_{i=1}^{3} \lambda_{i} \mathbf{u}_{\mathbf{i}} \mathbf{u}_{\mathbf{i}}^{\mathbf{H}}=\mathbf{T}_{\mathbf{0 1}}+\mathbf{T}_{\mathbf{0 2}}+\mathbf{T}_{\mathbf{0 3}} \\
\mathbf{C}=\mathbf{U} \boldsymbol{\Sigma} \mathbf{U}^{-1}=\sum_{i=1}^{3} \lambda_{i} \mathbf{u}_{\mathbf{i}} \mathbf{u}_{\mathbf{i}}^{\mathbf{H}}=\mathbf{C}_{\mathbf{0 1}}+\mathbf{C}_{\mathbf{0 2}}+\mathbf{C}_{\mathbf{0 3}}
\end{gathered}
$$

In (5.27), $\boldsymbol{U}$ is a matrix whose columns are eigenvectors of $\boldsymbol{T}, \boldsymbol{\Sigma}$ is a diagonal matrix that contains eigenvalues of $\boldsymbol{T}, \lambda_{i}$ is the eigenvalue of $\boldsymbol{T}$, and $\boldsymbol{u}_{\boldsymbol{i}}$ is the eigenvector of $\boldsymbol{T}$. Similar notations hold for covariance matrix equation in (5.28). In our study, we consider 3 decomposition methods from this class: Cloude decomposition [133], Holm decomposition [132], and van Zyl decomposition [134]. 
In Cloude decomposition, the component in (5.27) with the largest eigenvalue is taken as the scattering component of the target. Therefore, the target coherency matrix is given by (5.29), where $\lambda_{1}$ is the largest eigenvalue of $\boldsymbol{T}$.

$$
\mathbf{T}_{01}=\lambda_{1} \mathbf{u}_{1} \mathbf{u}_{1}^{\mathbf{H}}
$$

On the other hand, Holm decomposition assumes that the coherency matrix consists of single target with two remainder noise terms. Thus, the eigenvalue matrix $\Sigma$ can be written as in (5.30), where $\lambda_{1} \geq \lambda_{2} \geq \lambda_{3}$.

$$
\boldsymbol{\Sigma}=\left[\begin{array}{ccc}
\lambda_{1}-\lambda_{2} & 0 & 0 \\
0 & 0 & 0 \\
0 & 0 & 0
\end{array}\right]+\left[\begin{array}{ccc}
\lambda_{2}-\lambda_{3} & 0 & 0 \\
0 & \lambda_{2}-\lambda_{3} & 0 \\
0 & 0 & 0
\end{array}\right]+\left[\begin{array}{ccc}
\lambda_{3} & 0 & 0 \\
0 & \lambda_{3} & 0 \\
0 & 0 & \lambda_{3}
\end{array}\right]
$$

The first term in (5.30) represents the target component, which also satisfies rank 1 assumption. The second term represents a mixture between two minor scatterers, while the last term is equal to the coherency matrix of noise component. Finally, we can obtain the coherency matrix of the target as

$$
\mathbf{T}_{1}=\mathbf{U}\left[\begin{array}{ccc}
\lambda_{1}-\lambda_{2} & 0 & 0 \\
0 & 0 & 0 \\
0 & 0 & 0
\end{array}\right] \mathbf{U}^{-1}
$$

By contrast to the previous decomposition methods, van Zyl decomposition utilizes the covariance matrix $\boldsymbol{C}$ instead of the coherency matrix $\boldsymbol{T}$. This technique was developed based on the covariance matrix in most of natural terrain, where the correlation between copolarized and cross-polarized terms is almost zero. In such a case, the covariance matrix can be approximated as

$$
\mathbf{C}=\left[\begin{array}{ccc}
\left\langle\left|S_{X X}\right|^{2}\right\rangle & 0 & \left\langle S_{X X} S_{Y Y}^{*}\right\rangle \\
0 & \left\langle 2\left|S_{X Y}\right|^{2}\right\rangle & 0 \\
\left\langle S_{Y Y} S_{X X}^{*}\right\rangle & 0 & \left\langle\left|S_{Y Y}\right|^{2}\right\rangle
\end{array}\right]=\alpha\left[\begin{array}{ccc}
1 & 0 & \rho \\
0 & \eta & 0 \\
\rho * & 0 & \mu
\end{array}\right]
$$

where 


$$
\begin{aligned}
& \alpha=\left\langle\left|S_{X X}\right|^{2}\right\rangle \\
& \rho=\left\langle S_{X X} S_{Y Y}^{*}\right\rangle /\left\langle\left|S_{X X}\right|^{2}\right\rangle \\
& \eta=\left\langle 2\left|S_{X Y}\right|^{2}\right\rangle /\left\langle\left|S_{X X}\right|^{2}\right\rangle \\
& \mu=\left\langle\left|S_{Y Y}\right|^{2}\right\rangle /\left\langle\left|S_{X X}\right|^{2}\right\rangle
\end{aligned}
$$

The parameters $\alpha, \rho, \eta$, and $\mu$ are related to the size, shape, and electrical properties of the scatterers. Finally, the eigenvalues of such matrix can be derived in closed forms as

$$
\begin{aligned}
& \lambda_{1}=0.5 \alpha\left[1+\mu+\sqrt{(1-\mu)^{2}+4|\rho|^{2}}\right] \\
& \lambda_{2}=0.5 \alpha\left[1+\mu-\sqrt{(1-\mu)^{2}+4|\rho|^{2}}\right] \\
& \lambda_{3}=\alpha \eta
\end{aligned}
$$

As before, the decomposition results in this approach can be presented in RGB image. The color-codes for Cloude decomposition and Holm decomposition are red $=T_{22 T}$, green $=T_{33 T}$, and blue $=T_{11 T}$, where $T_{i j T}$ is the $(i, j)$-th element of target coherency matrix $\boldsymbol{T}_{\boldsymbol{0}}$. Meanwhile, in van Zyl decomposition we can assign red $=\lambda_{1}$, green $=\lambda_{2}$, and blue $=\lambda_{3}$.

\section{Model-Based Decomposition}

In model-based decomposition, the covariance matrix is assumed to comprise several fixed scattering models. This technique was originally developed by identifying natural scatterers found in many imaging scenarios. Although there are several decomposition methods that come from this class, we will only consider Freeman-Durden decomposition [135], [136], which is more relevant for GPR imaging.

Freeman-Durden decomposition proposes 3 main scattering mechanisms as the target models, i.e., surface scattering $\left(f_{S}\right)$, double-bounce scattering $\left(f_{D}\right)$, and volume scattering $\left(f_{V}\right)$. The covariance matrix $\boldsymbol{C}$ can be expressed in terms of these parameters as shown in (5.35), where $\alpha$ is the component from covariance matrix of double-bounce scattering, and $\beta$ is the component from covariance matrix of surface scattering.

$$
\mathbf{C}=\left[\begin{array}{ccc}
f_{S}|\beta|^{2}+f_{D}|\alpha|^{2}+0.375 f_{V} & 0 & f_{S} \beta+f_{D} \alpha+0.125 f_{V} \\
0 & 0.25 f_{V} & 0 \\
f_{S} \beta^{*}+f_{D} \alpha+0.125 f_{V} & 0 & f_{S}+f_{D}+0.375 f_{V}
\end{array}\right]
$$


The most straightforward method to solve (5.35) is by equating each element with the corresponding covariance matrix element. Unfortunately, these equations are underdetermined. To overcome such issue, additional assumption is made as follows. If the real part of $\left\langle S_{H H} S_{V V}{ }^{*}\right\rangle$ is positive, then the double-bounce scattering dominates and we set $\alpha=-1$. By assigning this value of $\alpha$ into (5.35), one can obtain the solutions given in (5.36).

$$
\begin{aligned}
& z_{3}=\frac{\left\langle S_{H H} S_{V V}{ }^{*}\right\rangle+\left\langle S_{V V} S_{V V}{ }^{*}\right\rangle-0.5 f_{V}}{\left\langle S_{H H} S_{H H}{ }^{*}\right\rangle-\left\langle S_{V V} S_{V V}{ }^{*}\right\rangle} \\
& y=\frac{\operatorname{Im}\left(z_{3}\right)}{\left|z_{3}\right|^{2}}\left(2 \operatorname{Re}\left(z_{3}\right)+1\right) \\
& x=-1+y \operatorname{Re}\left(z_{3}\right) / \operatorname{Im}\left(z_{3}\right) \\
& \beta=x+i y \\
& f_{S}=\frac{\left\langle S_{H H} S_{V V}{ }^{*}\right\rangle+\left\langle S_{V V} S_{V V}{ }^{*}\right\rangle-0.5 f_{V}}{\beta+1} \\
& f_{D}=\left\langle S_{V V} S_{V V}{ }^{*}\right\rangle-0.375 f_{V}-f_{S}
\end{aligned}
$$

However, if the real part of $\left\langle S_{H H} S_{V V}{ }^{*}\right\rangle$ is negative, then the surface scattering dominates and we set $\beta=1$. Then, we can calculate the other components by using the formulas given in (5.37).

$$
\begin{aligned}
& z_{3}=\frac{\left\langle S_{H H} S_{V V}{ }^{*}\right\rangle-\left\langle S_{V V} S_{V V}{ }^{*}\right\rangle+0.25 f_{V}}{\left\langle S_{H H} S_{H H}{ }^{*}\right\rangle-\left\langle S_{V V} S_{V V}{ }^{*}\right\rangle} \\
& y=-\frac{\operatorname{Im}\left(z_{3}\right)}{\left|z_{3}\right|^{2}}\left(2 \operatorname{Re}\left(z_{3}\right)-1\right) \\
& x=1+y \operatorname{Re}\left(z_{3}\right) / \operatorname{Im}\left(z_{3}\right) \\
& \alpha=x+i y \\
& f_{D}=\frac{\left\langle S_{H H} S_{V V}{ }^{*}\right\rangle+\left\langle S_{V V} S_{V V}{ }^{*}\right\rangle+0.25 f_{V}}{\alpha-1} \\
& f_{S}=\left\langle S_{V V} S_{V V}{ }^{*}\right\rangle-0.375 f_{V}-f_{D}
\end{aligned}
$$

Finally, the powers of each scattering mechanism are given by (5.38). These components can be visualized in color-coded image with red $=P_{D}$, green $=P_{V}$, and blue $=P_{S}$. 


$$
\begin{aligned}
& P_{S}=f_{S}\left(1+|\beta|^{2}\right) \\
& P_{D}=f_{D}\left(1+|\alpha|^{2}\right) \\
& P_{V}=f_{V}
\end{aligned}
$$

Besides Freeman-Durden decomposition, there are some others decomposition techniques belong to model-based decomposition approach. In [137] and [138], Yamaguchi proposed another model that counts the non-symmetric components of covariance matrix, which are assumed to be zero in Freeman-Durden decomposition. There are 4 scattering mechanisms in the target model of Yamaguchi decomposition: surface scattering, double-bounce scattering, volume scattering, and helix scattering. Meanwhile, simplified target models are proposed by Freeman in [139], where only 2 scattering mechanisms are considered: ground scattering and volume scattering. This method was originally developed for polarimetric SAR observations in forested area.

\subsubsection{H-Alpha Decomposition}

$\mathrm{H}$-alpha decomposition was first introduced as a classification methods based on 2 rollinvariant parameters, namely entropy and alpha angle [140]. Such parameters can be extracted from the eigen-value decomposition of coherency matrix as expressed in (5.39), where $\boldsymbol{U}$ is a matrix whose columns are eigenvectors of $\boldsymbol{T}, \boldsymbol{\Sigma}$ is a diagonal matrix that contains eigenvalues of $\boldsymbol{T}$, and $\lambda_{i}$ is the eigenvalue of $\boldsymbol{T}$.

$$
\mathbf{T}=\mathbf{U} \boldsymbol{\Sigma} \mathbf{U}^{-1}=\mathbf{U}\left[\begin{array}{ccc}
\lambda_{1} & 0 & 0 \\
0 & \lambda_{2} & 0 \\
0 & 0 & \lambda_{3}
\end{array}\right] \mathbf{U}^{-1}
$$

If $\boldsymbol{T}$ has only one non-zero eigenvalue, then this matrix corresponds to a pure target and we can easily obtain a single Sinclair matrix from $\boldsymbol{T}$. Meanwhile, if all its eigenvalues are equal, this matrix is said to be random and no polarization structure can be correlated with it. Between these two extremes, there exists a case of partial targets where the coherency matrix has nonzero and non-equal eigenvalues. Based on these properties, a statistical model of the scatterer is developed as a 3 symbol Bernoulli process with pseudo-probabilities $P_{i}$ given by

$$
P_{i}=\frac{\lambda_{i}}{\lambda_{1}+\lambda_{2}+\lambda_{3}}
$$


The entropy $(H)$ is formulated based on these pseudo-probabilities as expressed in (5.41). This parameter defines the degree of statistical disorder of the scatterers. If the entropy is low, then the target is a weakly depolarizing scatterer and the dominant scattering mechanism can be obtained. However, if the entropy is high, then the target is depolarizing and we are unable to extract a single dominant scatterer from the coherency matrix. In the extreme case where $H$ $=1$, the polarization information becomes zero and the target is purely a random noise processes. Since the eigenvalues are rotationally invariant, the entropy also possesses rollinvariant properties.

$$
H=\sum_{i=1}^{3}-P_{i} \log _{3} P_{i}
$$

Meanwhile, the alpha angle $(\bar{\alpha})$ can be obtained from parameterization of eigenvector matrix as given in (5.42). Each column of eigenvector matrix $\boldsymbol{U}$ represents certain scattering mechanism with occurrence probability defined by (5.40).

$$
\begin{aligned}
\mathbf{U} & =\left[\begin{array}{lll}
\mathbf{q}_{1} & \mathbf{q}_{2} & \mathbf{q}_{3}
\end{array}\right]^{T} \\
& =\left[\begin{array}{ccc}
\cos \alpha_{1} & \cos \alpha_{2} & \cos \alpha_{3} \\
\sin \alpha_{1} \cos \beta_{1} e^{j \delta_{1}} & \sin \alpha_{2} \cos \beta_{2} e^{j \delta_{2}} & \sin \alpha_{3} \cos \beta_{3} e^{j \delta_{3}} \\
\sin \alpha_{1} \cos \beta_{1} e^{j \gamma_{1}} & \sin \alpha_{2} \cos \beta_{2} e^{j \gamma_{2}} & \sin \alpha_{3} \cos \beta_{3} e^{j \gamma_{3}}
\end{array}\right]
\end{aligned}
$$

Following such assumption, the mean parameters of the dominant scattering mechanisms can be calculated as

$$
\overline{\mathbf{q}}=e^{i \varphi}\left[\begin{array}{c}
\cos \bar{\alpha} \\
\sin \bar{\alpha} \cos \bar{\beta} e^{i \bar{\delta}} \\
\sin \bar{\alpha} \cos \bar{\beta} e^{i \bar{\gamma}}
\end{array}\right]
$$

where $\varphi$ is the absolute target phase, and parameters $\bar{\alpha}, \bar{\beta}, \bar{\delta}$, and $\bar{\gamma}$ are given by (5.44).

$$
\begin{aligned}
& \bar{\alpha}=\sum_{i=1}^{3} \alpha_{i} P_{i} \\
& \bar{\beta}=\sum_{i=1}^{3} \beta_{i} P_{i} \\
& \bar{\delta}=\sum_{i=1}^{3} \delta_{i} P_{i} \\
& \bar{\gamma}=\sum_{i=1}^{3} \gamma_{i} P_{i}
\end{aligned}
$$


It can be shown that among these parameters, only $\bar{\alpha}$ that has roll-invariant properties. This angle can be related to the shape of the target, in which there is a continuous change of alpha angle value from surface scatterer, through dipole scatterer, until double bounce scatterer.

Based on the described properties of $H$ and alpha angle, Cloude and Pottier [140] proposed an unsupervised classification scheme of random target by which the $\mathrm{H}$-alpha plane is divided into 9 classification zones as presented in Fig. 5.2. This technique has been verified initially by analysis on San Fransisco Bay PolSAR data, and further extended into various cases of terrain and land-use classification.

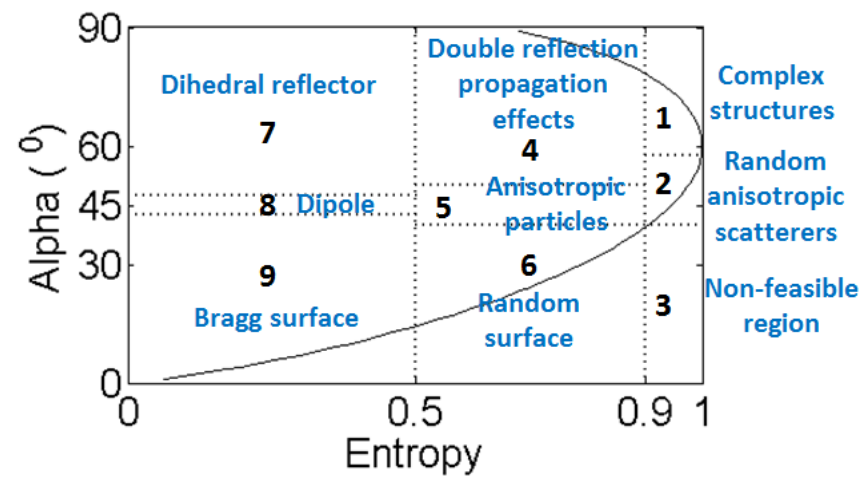

Fig. 5.2: Zone classification on H-alpha plane.

\subsection{Proposed Polarization Diversity Method for UWB GPR}

Although polarimetric technique was originally developed for remote sensing, some recent works on GPR have move towards the utilization of polarimetric method. Several studies [8], [141]-[144] have demonstrated the advantages of dual-polarization measurement in capturing various target shapes and orientations. Some works in [145] and [146] demonstrated the utilization of fully-polarimetric FMCW GPR. Classification techniques for subsurface objects by using polarization anisotropy coefficient and polarimetric signature were proposed therein. In [22], a polarimetric processing technique was developed for landmine characterization. Another research in [51] and [52] employs fully-polarimetric measurement for building inspection after earthquake. It was shown therein that fully-polarimetric data can provide more information on damages inside a wooden structure. Some of polarimetric decomposition techniques in PolSAR have also been applied for GPR system. In [71] and [72], Freeman-Durden and H-alpha decomposition methods were used to distinguish several basic target shapes in GPR data. Other research related to bore-hole radar exploited Freeman- 
Durden [75], Pauli, and H-alpha decompositions [74] for fracture characterization. Motivated by all above-mentioned works, in this study we conduct further exploration on various polarimetric decomposition methods with focus on utilities mapping application. The fullypolarimetric system and the signal processing methods are described in the following.

\subsubsection{Fully-Polarimetric UWB GPR System}

The fully-polarimetric mode is implemented by simply collecting the data in combinations of 2 orthogonal polarization bases. Typically, the existing systems use either linear polarization or circular polarization. However, circular polarization is hard to maintain over wide bandwidth, so linear polarization is preferable in practice. In our system, we utilize 2 orthogonal linear polarization bases which are aligned with $x$-and $y$-axes of the Cartesian coordinate. The measurement configuration is similar to Fig. 3.1 in Subsection 3.1.2. For each measurement, we obtain 4 datasets labeled as $\mathrm{XX}, \mathrm{XY}, \mathrm{YX}$, and YY, where the first term denotes the transmitter polarization and the second term denotes the receiver polarization. Although the study is conducted on linear polarization bases, the results will also hold for another polarization bases in ideal case. In addition, we can apply transformation of polarization bases to the Sinclair, coherency, and covariance matrices as given in (5.4), (5.9), and (5.10) respectively.

We limit the study of fully-polarimetric GPR into monostatic case only. In the simulation, we use collocated transmitter and receiver, while in the experiment configuration we set the distance between the transmitter and the receiver to be relatively small compared to the distance from the antennas to the ground. Therefore, we assume the cross-polarized components $\mathrm{XY}$ and $\mathrm{YX}$ to be equal. In practice, such assumption does not always hold, and the following issues need to be considered to handle the bistatic case. Firstly, the reciprocity property does not hold in bistatic case, and thus the cross-polarized components are unequal. Meanwhile, the polarimetric decomposition methods that we have introduced so far are initially developed for PolSAR, which use the monostatic assumption. To the author knowledge, most of these methods does not have the equivalent bistatic versions. Nevertheless, some decomposition methods can be generalized into bistatic case. For instance, the Sinclair matrix can be decomposed into 4 Pauli matrices as given in (5.45). The H-alpha decomposition can be modified by using $4 \times 4$ coherency matrix as given in (5.46). The second issue is related to the system. The bistatic measurement yields non-zero angle of incident in the scattering, which can alter the magnitude of the received fields on each polarization basis. 
Therefore, system calibration need to be performed before applying the decomposition methods.

$$
\begin{gathered}
\mathbf{S}=\left[\begin{array}{cc}
S_{X X} & S_{X Y} \\
S_{Y X} & S_{Y Y}
\end{array}\right]=\frac{a}{\sqrt{2}}\left[\begin{array}{ll}
1 & 0 \\
0 & 1
\end{array}\right]+\frac{b}{\sqrt{2}}\left[\begin{array}{cc}
1 & 0 \\
0 & -1
\end{array}\right]+\frac{c}{\sqrt{2}}\left[\begin{array}{cc}
0 & 1 \\
1 & 0
\end{array}\right]+\frac{d}{\sqrt{2}}\left[\begin{array}{cc}
0 & -i \\
i & 0
\end{array}\right] \\
\mathbf{T}=\left\langle\mathbf{k} \mathbf{k}^{H}\right\rangle \\
\mathbf{k}=\frac{1}{\sqrt{2}}\left[\begin{array}{ll}
S_{X X}+S_{Y Y} & \left.S_{X X}-S_{Y Y} \quad S_{X Y}+S_{Y X} \quad j\left(S_{X Y}+S_{Y X}\right)\right]^{T}
\end{array}\right.
\end{gathered}
$$

Although the study is carried out mainly in SISO configuration, we will also explore the polarization characteristic of MIMO sensor configuration. In particular, we perform comparative study between SISO configuration and MIMO configuration based on the output of Pauli decomposition and $\mathrm{H}$-alpha decomposition.

\subsubsection{Implementation of PolSAR Decomposition Method on UWB GPR Data}

The signal processing steps of polarimetric GPR are presented in Fig. 5.3. Each polarimetric data is first preprocessed and migrated independently. We employ $\mathrm{f}-\mathrm{k}$ migration for simulation data due to the ideal sensor configuration, and Kirchhoff migration for experimental data.

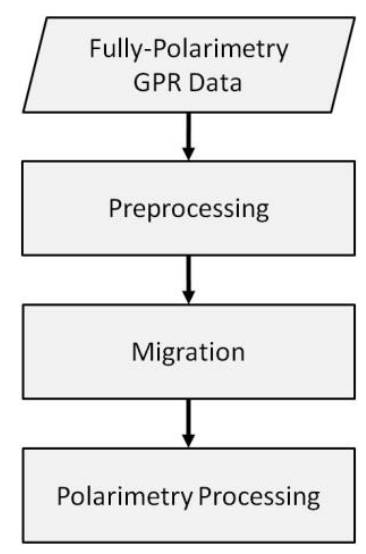

Fig. 5.3: Signal processing method on polarimetric GPR.

As described in Section 5.1, the coherent decomposition methods are performed on Sinclair matrix. We can extract such term by arranging the migration output into $2 \times 2$ matrix as given in (5.48), where $U_{X X}, U_{X Y}, U_{Y X}$, and $U_{Y Y}$ are the migration results of $\mathrm{XX}, \mathrm{XY}, \mathrm{YX}$, and $\mathrm{YY}$ data respectively. 


$$
\mathbf{S}=\left[\begin{array}{ll}
U_{X X} & U_{X Y} \\
U_{Y X} & U_{Y Y}
\end{array}\right]
$$

On the other hand, the incoherent decomposition methods are based on second order statistics of Sinclair matrix, namely coherency matrix and covariance matrix. We calculate these matrices by taking average of $\boldsymbol{T}_{\boldsymbol{i}}$ and $\boldsymbol{C}_{\boldsymbol{i}}$ within a box of size $2.5 \times 2.5 \times 2.5 \mathrm{~cm}$, where $\boldsymbol{T}_{\boldsymbol{i}}$ and $\boldsymbol{C}_{\boldsymbol{i}}$ are the covariance and coherency matrices of individual pixel-i respectively as given in (5.49) and (5.50). Finally, we apply the PolSAR decomposition methods to the Sinclair, coherency, and covariance matrices.

$$
\begin{aligned}
& \mathbf{T}_{\mathbf{i}}=\mathbf{k}_{\mathbf{i}} \mathbf{k}_{\mathbf{i}}{ }^{H} \quad \text { with } \mathbf{k}_{\mathbf{i}}=\frac{1}{\sqrt{2}}\left[\begin{array}{lll}
S_{X X}+S_{Y Y} & S_{X X}-S_{Y Y} \quad 2 S_{X Y}
\end{array}\right]^{T} \\
& \mathbf{C}=\boldsymbol{\Omega}_{\mathbf{i}} \boldsymbol{\Omega}_{\mathbf{i}}{ }^{H} \quad \text { with } \boldsymbol{\Omega}_{\mathbf{i}}=\left[\begin{array}{lll}
S_{X X} & \sqrt{2} S_{X Y} & S_{Y Y}
\end{array}\right]^{T}
\end{aligned}
$$

\subsection{Performance of Polarimetric UWB GPR Methods on Simulation Data}

We begin the study by investigating the performance of various PolSAR decomposition techniques on simulation data generated by GPRMax. The data acquisition is performed by using SISO array which contains 9 pairs of collocated transmitter and receiver. This array is elongated along $x$-axis and moves in the direction of $y$-axis with spatial sampling of $1 \mathrm{~cm}$. The distance between each pair of sensor is $7.5 \mathrm{~cm}$, while the distance of the antenna from the ground surface is $10 \mathrm{~cm}$. The transmitted waveform is a $1^{\text {st }}$ Gaussian derivative with center frequency of $1 \mathrm{GHz}$.

We divide the evaluation into 2 parts. In the first part, we analyze the performance of the decomposition methods in distinguishing several basic target shapes. Since our focus is on utilities mapping application, we propose several target models which are common in such application. In the second part, we aim to observe the response of the decomposition methods over variation in target size. Specifically, we investigate the responses of those methods on cylinders with various diameters.

\subsubsection{Response of Decomposition Methods on Variation of Target Shapes}

The target models are provided in Fig. 5.4. The first target in Fig. 5.4(a) is a PEC plate with size $60 \times 40 \mathrm{~cm}$ and thickness $1 \mathrm{~cm}$, while the second targets in Fig. 5.4(b) are rebars with diameter $1.5 \mathrm{~cm}$. The last target is a PEC dihedral with size $80 \times 25 \mathrm{~cm}$. In all these 
simulations, the subsurface medium has relative permittivity 6 , relative permeability 1 , and conductivity $0.001 \mathrm{~S} / \mathrm{m}$.

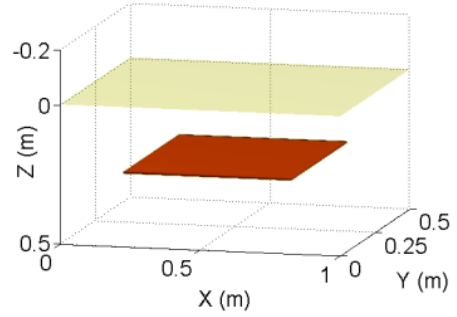

(a) Plate

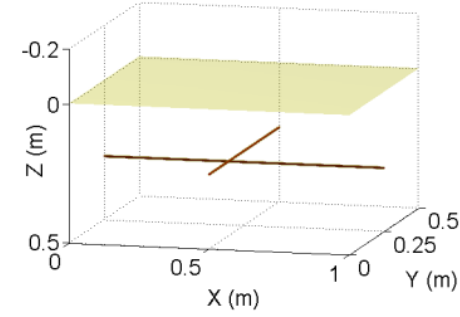

(b) Rebars

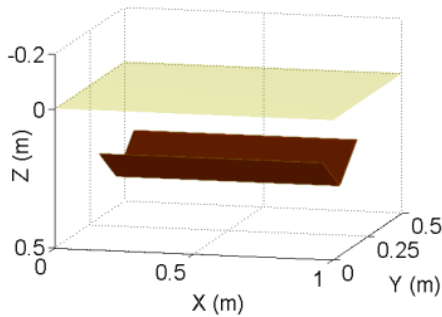

(c) Dihedral

Fig. 5.4: Simulation target models.

After the data is generated, the preprocessing and migration are applied independently on each polarization datasets. The results are given in Fig. 5.5, where the surfaces are plotted with amplitude ranging from $-10 \mathrm{~dB}$ to $-3 \mathrm{~dB}$. The $\mathrm{HH}, \mathrm{VV}$, and $\mathrm{HV} / \mathrm{VH}$ components are plotted in red, blue, and yellow color respectively.

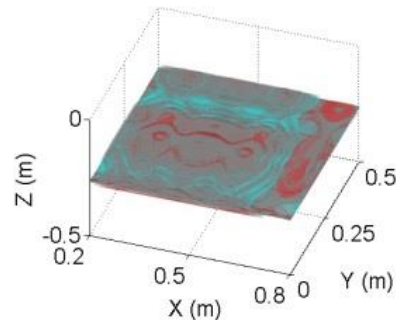

(a) Plate

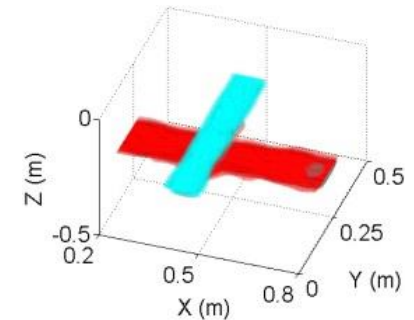

(b) Rebars

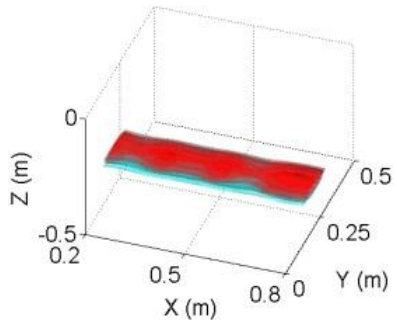

(c) Dihedral

Fig. 5.5: Migration results of the simulation data.

Finally, polarimetric decompositions are performed to combine these datasets. The results are presented in Table 5.1 for all the decomposition methods described in Section 5.1. As we can see, most of the decomposition methods provide color information regarding the target shape. The plate target is dominated by single scattering component, while the dihedral target is dominated by double-bounce scattering component. The rebars yields combination between single-bounce and double-bounce scattering components, since such target has similar polarization characteristic as a dipole. Moreover, we can observe that the H-alpha decomposition results of these targets satisfy the classification zone in Fig. 5.2, which was originally developed for PolSAR data. The plate target lies in the Bragg surface zone, the rebars are in the dipole zone, and the dihedral is in the dihedral reflector zone.

These results are mainly in agreement with the theoretical polarimetric characteristic of the corresponding targets. This can be shown by substituting the theoretical Sinclair matrices of 
each target to the formulas of each decomposition technique, which are described in Section 5.1. The Sinclair matrices of these targets can be formulated by (5.51), (5.52), and (5.53) for plate, dipole, and dihedral respectively.

$$
\begin{aligned}
& \mathbf{S}=\left[\begin{array}{ll}
1 & 0 \\
0 & 1
\end{array}\right] \\
& \mathbf{S}=\left[\begin{array}{ll}
1 & 0 \\
0 & 0
\end{array}\right] \text { for dipole along x-axis, } \mathbf{S}=\left[\begin{array}{ll}
0 & 0 \\
0 & 1
\end{array}\right] \text { for dipole along y-axis } \\
& \mathbf{S}=\left[\begin{array}{cc}
1 & 0 \\
0 & -1
\end{array}\right]
\end{aligned}
$$

We can also observe that some of the incoherent decompositions produce slightly different response on particular targets. The most obvious cases are Holm and van Zyl decompositions, which do not exhibit any color information on these target shapes. We can relate this result with the basic principle used in each decomposition methods. In particular, Holm decomposition utilizes eigen-value decomposition to separate the coherency matrix into a target component and 2 noise components. Meanwhile, van Zyl decomposition was developed to interpret the eigen-values of the covariance matrix for azimuthally symmetrical natural terrain. Since these algorithms do not give interpretation on scattering mechanism of deterministic targets, both of them yield the same color for all target shape in Fig. 5.5.

On the other hand, Freeman-Durden, Pauli, and Krogager decomposition algorithms decompose the coherency and Sinclair matrices into several basic scattering mechanisms, which can also be applied to interpret deterministic target. Huynen, Barnes-Holm, and Cloude decompositions are based on assumption of a pure target and distributed N-target. However, they utilize the coherency matrix components of the pure target that are related to the basic scattering mechanisms. Thus, such decomposition methods are still applicable for deterministic target. Finally, H-alpha decomposition utilizes the parameters of coherency matrix to obtain the dominant scattering mechanism, which is represented by alpha angle $(\bar{\alpha})$. This alpha angle can be used to interpret the shape of deterministic targets. 
Table 5.1: Results OF POLARIMETRIC DECOMPOSITION METHODS ON VARIOUS TARGET SHAPES

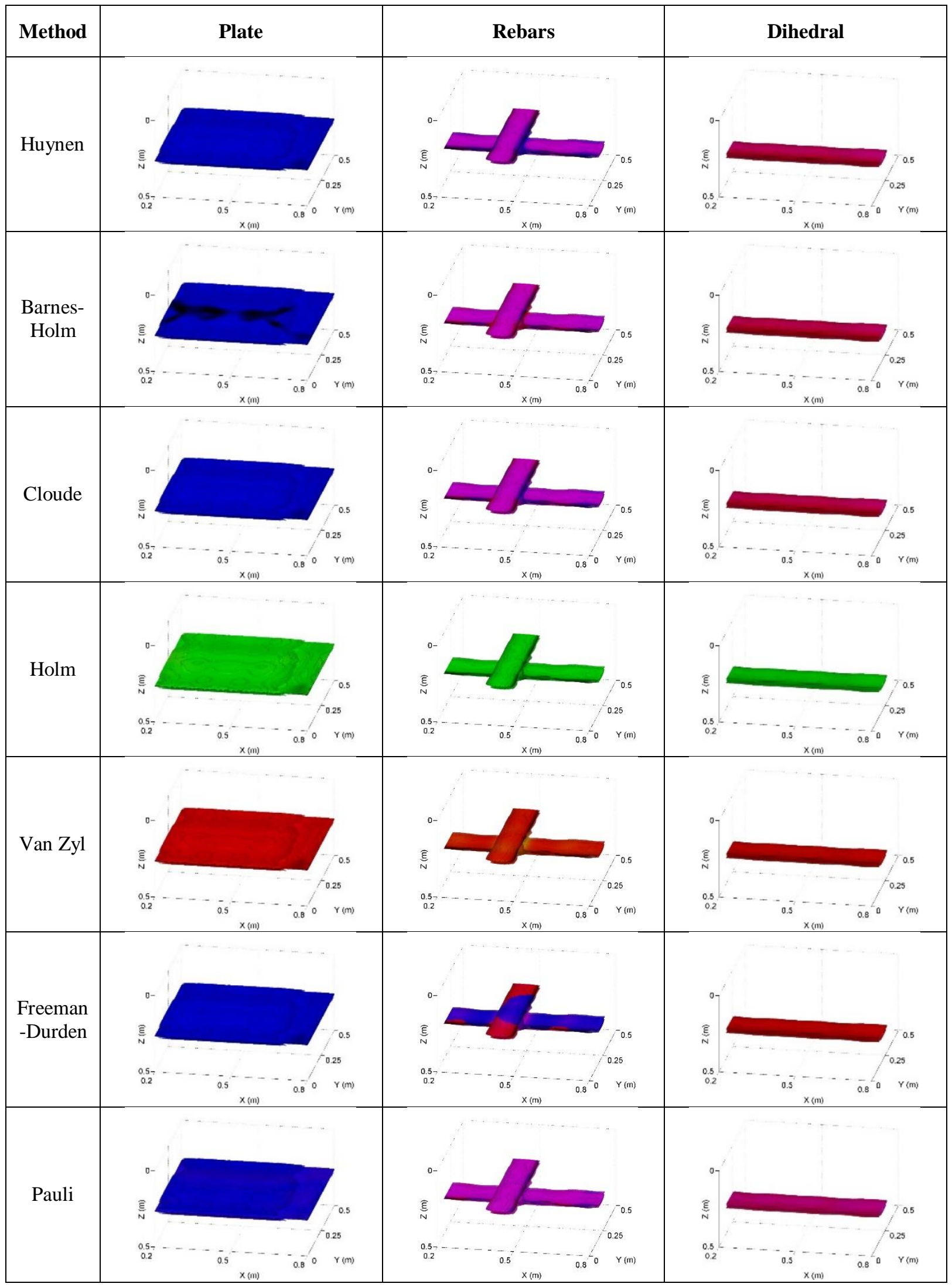




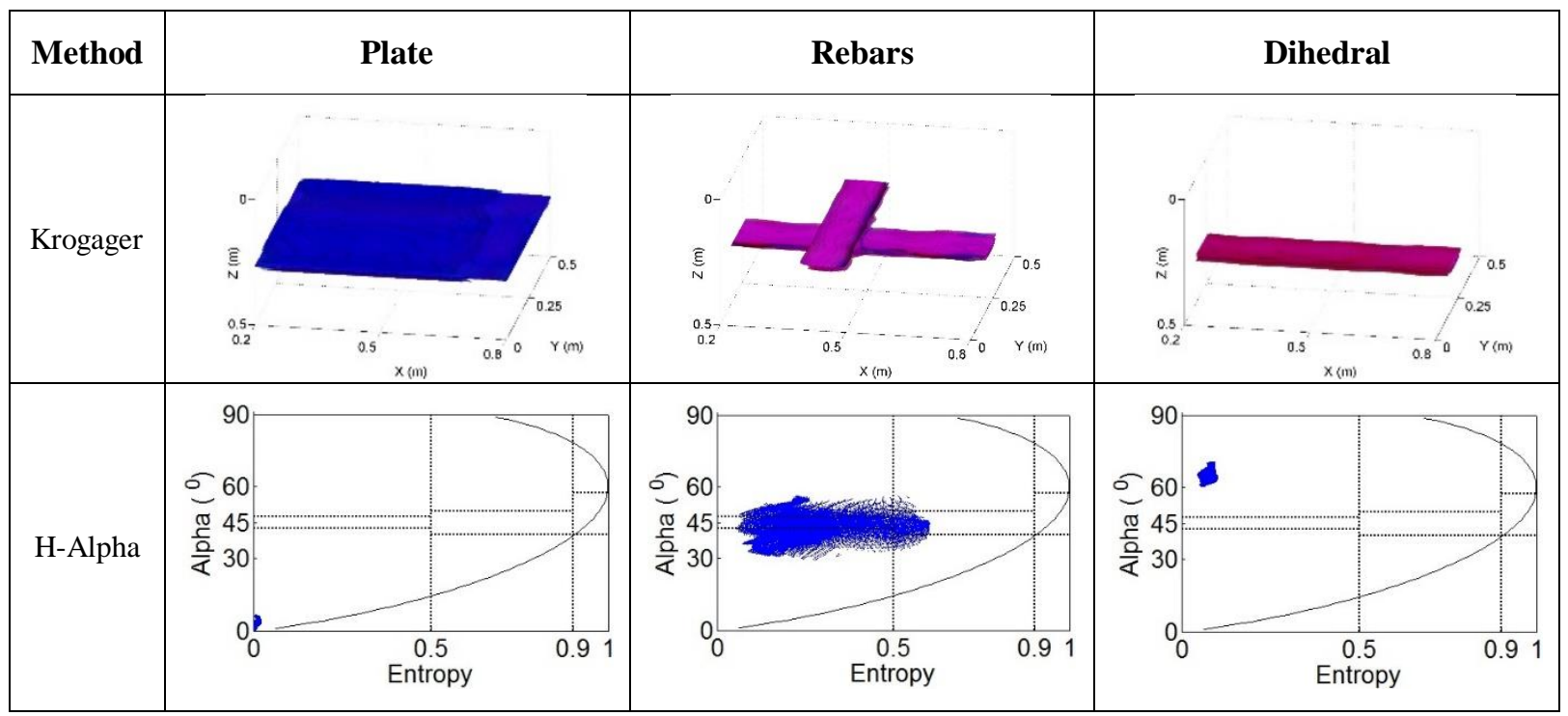

\subsubsection{Response of Decomposition Methods on Variation of Target Size}

We have seen previously that the rebars target yields similar polarimetric characteristic to a dipole. However, a target can be considered as dipole if its size is notably smaller than the wavelength of the transmitted wave. In utilities mapping, some cylindrical targets like pipes or cables have larger diameter, and thus may produce different polarimetric response. Such properties can be advantageous in providing more information regarding target size. Therefore, in this subsection, we observe the responses of the polarimetric decomposition methods on variation of cylinder size. The target models are PEC cylinders with diameters ranging from $2 \mathrm{~cm}$ to $8 \mathrm{~cm}$ as given in Fig. 5.6. In all these simulations, the subsurface medium has relative permittivity 6 , relative permeability 1 , and conductivity $0.001 \mathrm{~S} / \mathrm{m}$.

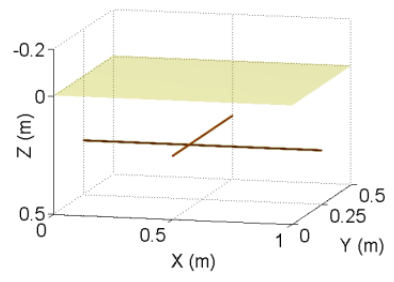

(a) Pipes with diameter $1 \mathrm{~cm}$

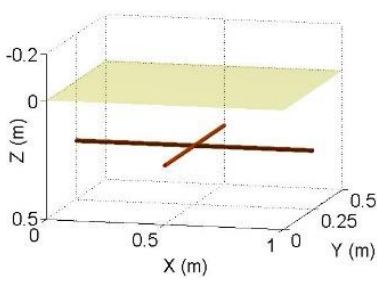

(b) Pipes with diameter $2 \mathrm{~cm}$

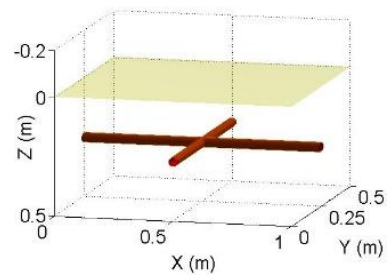

(c) Pipes with diameter $4 \mathrm{~cm}$

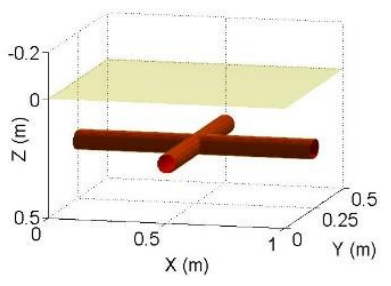

(d) Pipes with diameter $8 \mathrm{~cm}$

Fig. 5.6: Simulation target models.

We follow similar processing methods as presented in Subsection 5.3.1. The results are provided in Table 5.2. We have seen previously that except in Holm, van Zyl, and FreemanDurden decompositions, the dipole target in the rest decomposition methods appears in purple color, while the plate target has blue color. Referring to such properties, in Table 5.2 we can observe the transition in target color from purple to blue as the diameter of the 
cylinder increases. The H-alpha decomposition results also indicate such properties, in which the alpha angle values of the target move gradually from the dipole zone to the Bragg surface zone. These transitions imply that a cylinder target can behave as dipole scatterer or surface scatterer depends on its size.

By assuming the bandwidth of the signal is around $3 \mathrm{GHz}$, we can calculate the resolution over $y$-axis to be approximately $2 \mathrm{~cm}$. Meanwhile, the resolution over $x$-axis should be lower due to its larger spatial sampling. In Table 5.2, one can see that the smallest cylinder with diameter of $1 \mathrm{~cm}$ exhibits dipole characteristics since its diameter is significantly less than the resolution in both axes.

By contrast, the $2 \mathrm{~cm}$ cylinders totally appear in blue color on the images of Huynen and Cloude decompositions. In Barnes-Holm decomposition, some cylinder part that lies over $y$ axis still yields slightly purple color, while the part that elongates over $x$-axis already changes into blue. Such color difference occurs since the resolution over $x$-axis is lower than the resolution over $y$-axis. The cylinder diameter is still smaller than the $x$-axis resolution, while it is already comparable to the $y$-axis resolution. Meanwhile, in the coherent decomposition images the two orthogonal cylinders still produce purple color.

Finally, Huynen, Barnes-Holm, and Cloude decompositions exhibit blue color for both $4 \mathrm{~cm}$ and $8 \mathrm{~cm}$ cylinders. On the other hand, the coherent decomposition images show that the purple color gradually diminishes as the cylinder diameter becomes larger. These results demonstrate that these decomposition methods provide different progress on the color transition of the cylinder. In general, we can see that these properties can be used to roughly estimate the size of the cylinder.

Table 5.2: Results of POLARIMETRIC DECOMPOSITION METHODS ON VARIOUS TARGET SizES

\begin{tabular}{|c|c|c|c|c|}
\hline Method & $\begin{array}{l}\text { Pipes with diameter } \\
\qquad 1 \mathrm{~cm}\end{array}$ & $\begin{array}{l}\text { Pipes with diameter } \\
\qquad 2 \mathrm{~cm}\end{array}$ & $\begin{array}{l}\text { Pipes with diameter } \\
\qquad 4 \mathrm{~cm}\end{array}$ & $\begin{array}{l}\text { Pipes with diameter } \\
\qquad 8 \mathrm{~cm}\end{array}$ \\
\hline Huynen & 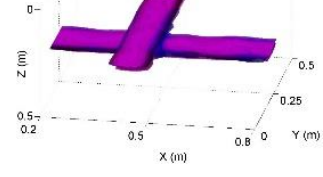 & $\underbrace{}_{\substack{0.5 \\
0.2}}$ & 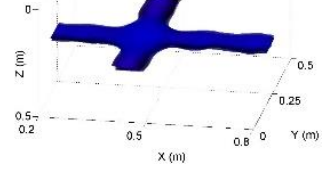 & 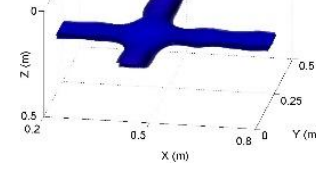 \\
\hline
\end{tabular}




\begin{tabular}{|c|c|c|c|c|}
\hline Method & $\begin{array}{l}\text { Pipes with diameter } \\
\qquad 1 \mathrm{~cm}\end{array}$ & $\begin{array}{l}\text { Pipes with diameter } \\
\qquad 2 \mathrm{~cm}\end{array}$ & $\begin{array}{l}\text { Pipes with diameter } \\
\qquad 4 \mathrm{~cm}\end{array}$ & $\begin{array}{l}\text { Pipes with diameter } \\
\qquad 8 \mathrm{~cm}\end{array}$ \\
\hline $\begin{array}{c}\text { Barnes- } \\
\text { Holm }\end{array}$ & 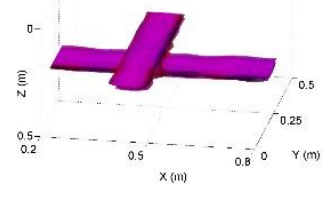 & $\underbrace{0.5}_{\substack{0.5 \\
0.5 \\
0.2}}$ & $\underset{\substack{0.5-2 \\
0.2}}{2}$ & ב- \\
\hline Cloude & $\underbrace{}_{\substack{0.5 \\
0.52 \\
0.2}}$ & $\underbrace{0.5}_{0.5}$ & 我 & 佥 \\
\hline Holm & 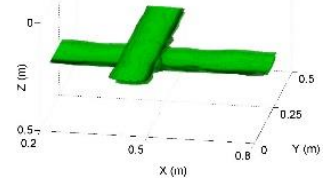 & $\overbrace{\substack{0.5 \\
0.5}}$ & 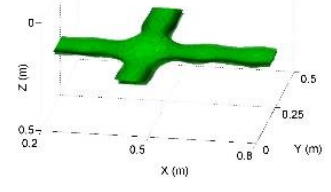 & $\int_{0.25}$ \\
\hline Van Zyl & ${ }_{\substack{0.5 \\
\text { s.5. } \\
0.2}}$ & 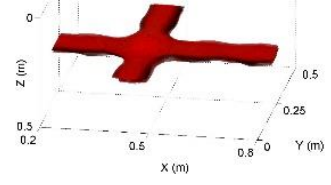 & 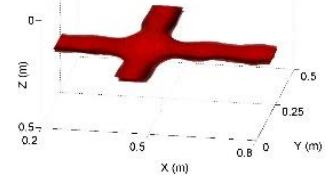 & $\begin{array}{l}\text { 佥 } \\
\text { o.s. } \\
0.2\end{array}$ \\
\hline $\begin{array}{l}\text { Freeman } \\
\text {-Durden }\end{array}$ & $\underbrace{}_{\substack{\mathrm{s} \\
0.5,2 \\
0.2}}$ & $\overbrace{\substack{0.5 \\
0.2}}$ & $\overbrace{\substack{0.5 \\
N}}$ & 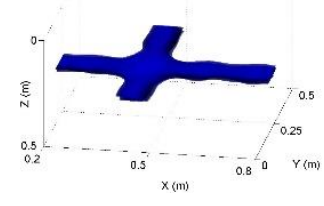 \\
\hline Pauli & $\underbrace{}_{\substack{0.5 \\
\text { o.s. } \\
0.2}}$ & 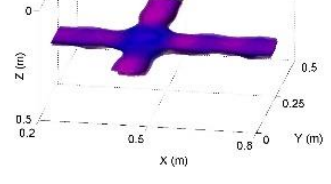 & $\int_{\int_{0.5}^{0.5}}^{0.5}$ & 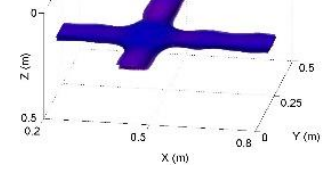 \\
\hline Krogager & $\underbrace{}_{\substack{0.5 \\
0.5,5 \\
0.2}}$ & 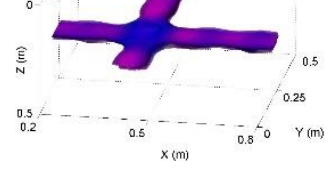 & $\underbrace{0.5}_{\int_{0.5}^{0.5}}$ & $\int_{0.025}$ \\
\hline
\end{tabular}




\begin{tabular}{|c|c|c|c|c|}
\hline Method & $\begin{array}{c}\text { Pipes with diameter } \\
1 \mathrm{~cm}\end{array}$ & $\begin{array}{l}\text { Pipes with diameter } \\
\qquad 2 \mathrm{~cm}\end{array}$ & $\begin{array}{l}\text { Pipes with diameter } \\
\qquad 4 \mathrm{~cm}\end{array}$ & $\begin{array}{c}\text { Pipes with diameter } \\
\qquad 8 \mathrm{~cm}\end{array}$ \\
\hline H-Alpha & ${ }_{0}^{60}$ & $\underbrace{\delta_{0} 60}_{0}$ & 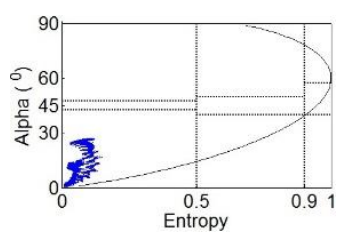 & 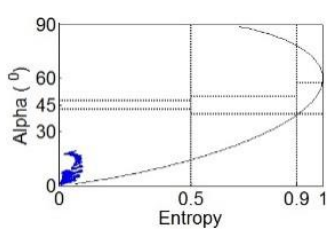 \\
\hline
\end{tabular}

\subsection{Performance of Polarimetric UWB GPR Methods on Experimental Data}

We have investigated the performance of each decomposition method by using simulation data. To verify those results, in this part we present the analysis on experimental data. We use experiment configuration shown in Fig. 5.7, the parameters of which are based on existing research work in GPR [147]. A pair of antennas is lifted with height of $1 \mathrm{~m}$ from the ground surface. These antennas are carried by a robotic arm which has very high positioning precision. Each antenna has 2 orthogonal linear polarizations in $x$ - and $y$-axes, and distance between the antennas is around $22 \mathrm{~cm}$. 


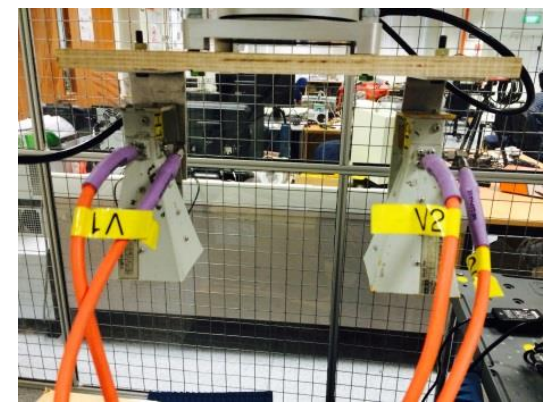

(a) Antenna system

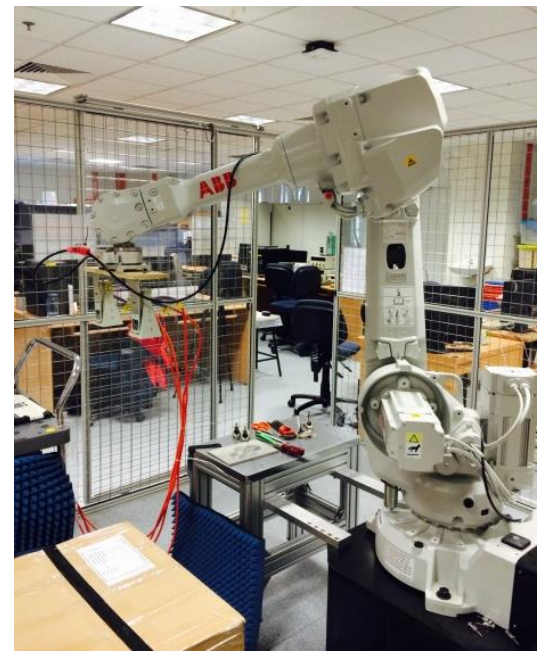

(c) Robotic arm for positioning

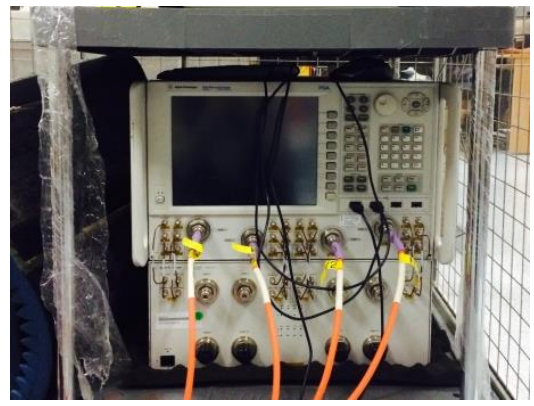

(b) Vector Network Analyzer

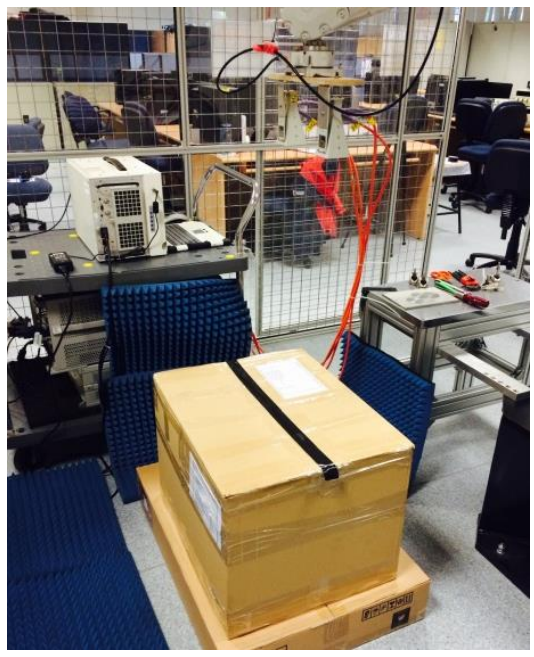

(d) Measurement domain

Fig. 5.7: Experiment configuration.

The waveform source is a vector network analyzer (VNA). The measurement is performed in SFCW mode, where the frequencies of the transmitted signal are over 3-6 GHz with 401 sampling point. We perform measurement point-by-point with sampling steps of $2.5 \mathrm{~cm}$. There are $20 \times 20$ points collected over XY plane.

The target configuration is given in Fig. 5.8, which comprises two metal pipes in orthogonal direction. The length of each pipe is $30 \mathrm{~cm}$ with diameter of $2 \mathrm{~cm}$. These targets are placed inside a box of size $50 \times 50 \times 50 \mathrm{~cm}$ filled with low dielectric constant material. 


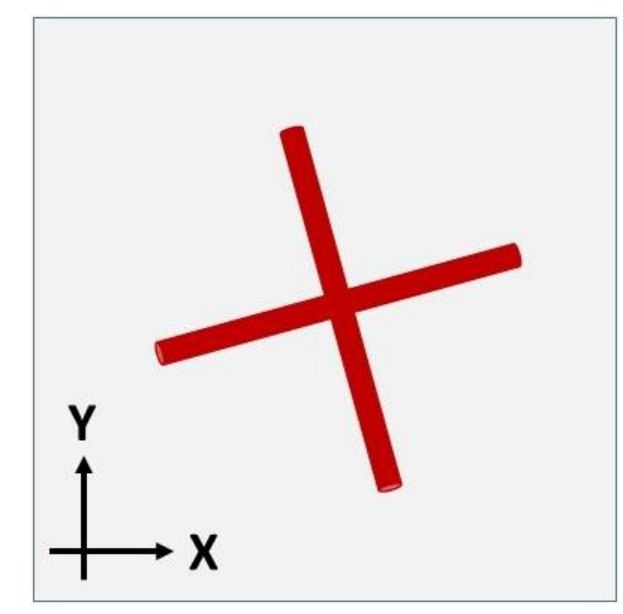

Fig. 5.8: Target configurations in the experiment.

The signal processing consists of 3 main steps. The first step is preprocessing, where the ground reflection and DC components are removed based on averaging method. The second step is migration. We use Kirchhoff migration in this case due to its flexibility in various sensor configurations. The result after migration steps is shown in Fig. 5.9, where the surface plots are taken with amplitude ranging from $-10 \mathrm{~dB}$ to $-3 \mathrm{~dB}$. The $\mathrm{XX}, \mathrm{YY}$, and $\mathrm{XY} / \mathrm{YX}$ polarized data are represented by red, blue, and yellow colors respectively.

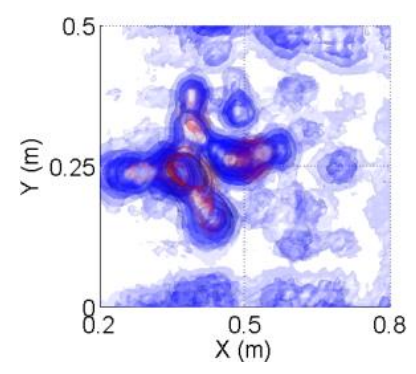

Fig. 5.9: Migration result on experimental data.

The last step is polarimetric decompositions. The results are provided in Table 5.3. These results are in agreement with the simulation results in which the pipes exhibit dipole polarization characteristics. We can also calculate the resolution of this system to be around 5 $\mathrm{cm}$, which is significantly higher than the diameter of the pipes. Therefore, the pipes polarimetric behavior also verifies the earlier simulation results on various target size presented in Subsection 5.3.2. Furthermore, by comparing these results to Fig. 5.9, we can see that some clutter and noise components are removed in incoherent polarimetric decomposition, while such components remain in the coherent decomposition images due to the direct utilization of Sinclair matrix. Similar to the simulation case, Holm and van Zyl 
decompositions do not provide color information regarding the shape of the target. However, all these decompositions still remove the clutter and the noise components in the original migration results.

Table 5.3: Results of POLARIMETRIC DECOMPOSITION METHOdS ON EXPERIMENTAL DATA

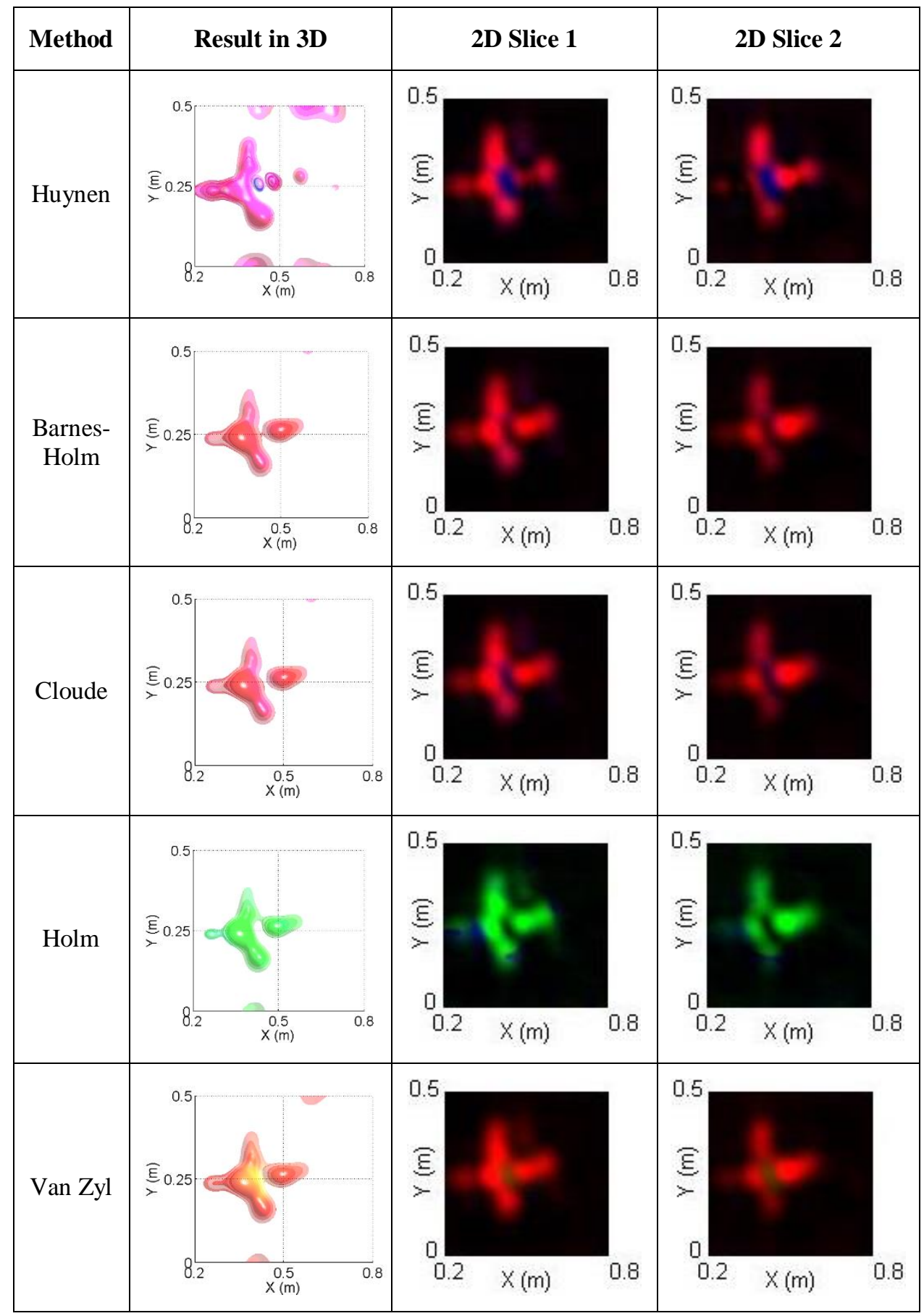




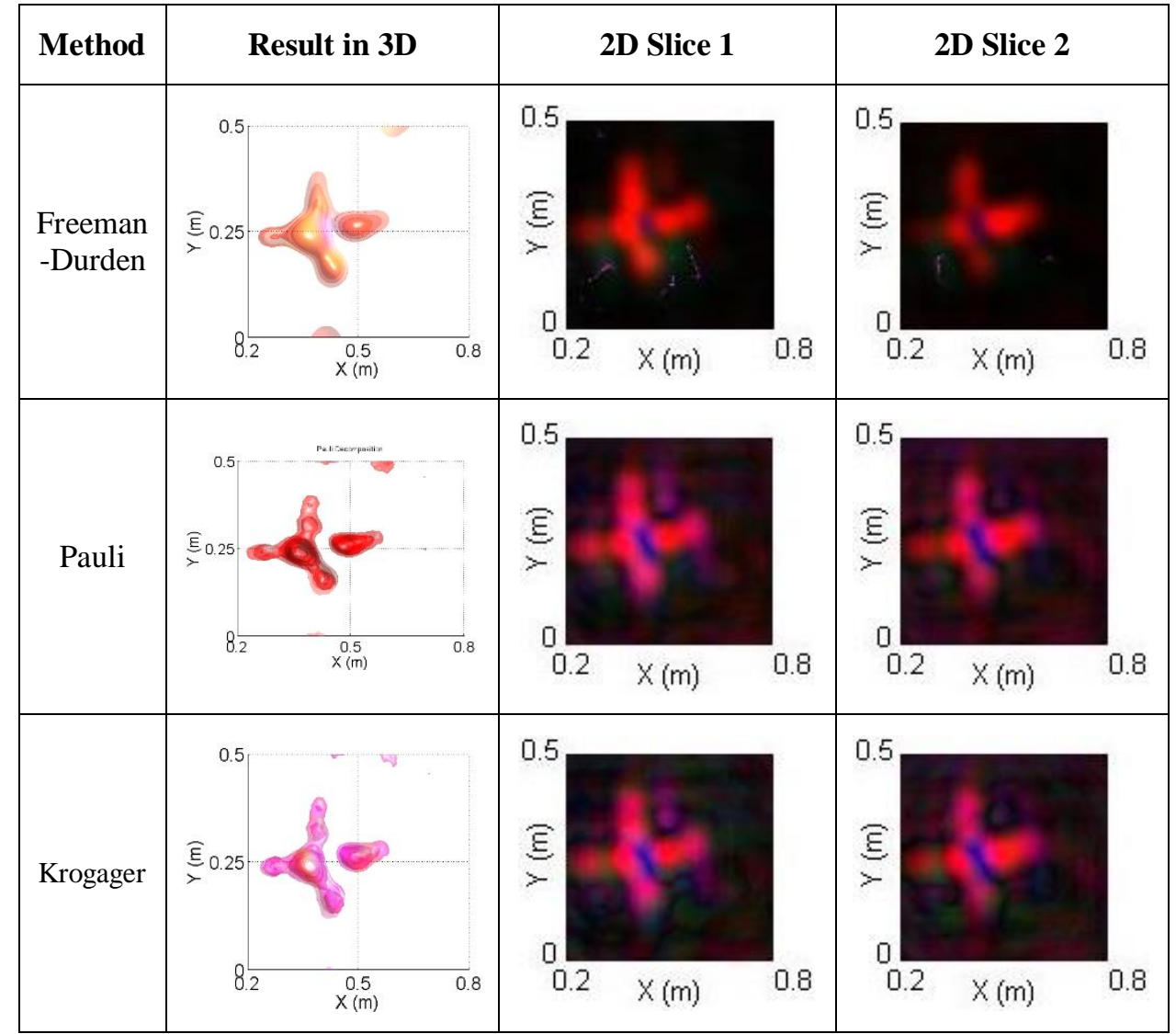

\subsection{Comparison of Polarimetric Characteristic of SISO and MIMO Configurations}

We have demonstrated in Chapter 3 that MIMO configuration offers better imaging capability compared to SISO and SIMO configurations. In this section, we aim to explore the effect of MIMO configuration on polarimetric characteristic of the target. Specifically, we evaluate the polarimetric output of SISO and MIMO configurations by using Pauli decomposition and H-alpha decomposition. The analysis is carried out based on a series of simulation scenarios related to utilities mapping and infrastructure inspection applications.

The target configurations are presented in Fig. 5.10. The first 4 targets represent some common shapes that can be encountered in utilities mapping, while the last 4 targets correspond to some defects that can be found during inspection of infrastructure. The plate target in Fig. 5.10(a) has size of $60 \times 40 \mathrm{~cm}$ and thickness of $1 \mathrm{~cm}$. The second targets are 2 pipes in orthogonal orientation with diameter of $10 \mathrm{~cm}$. The third targets are rebars with diameter of $1.5 \mathrm{~cm}$. The fourth target is a dihedral with size of $80 \times 25 \mathrm{~cm}$. These targets are made of PEC. Meanwhile in the lower part of Fig. 5.10, the fifth target is a planar void with size of $60 \times 50 \mathrm{~cm}$ and thickness of $1 \mathrm{~cm}$. The sixth target is a horizontal void crack generated 
by fractal model with fractal dimension of 2 and fractal range of $8 \mathrm{~cm}$. The next target in Fig. $5.10(\mathrm{~g})$ is a tilted version of the horizontal void crack with inclination angle of $30^{\circ}$ relative to the horizontal plane. Finally, the last target is a fractal volume made of free space with size of $25 \times 25 \times 25 \mathrm{~cm}$ and fractal dimension of 2 .

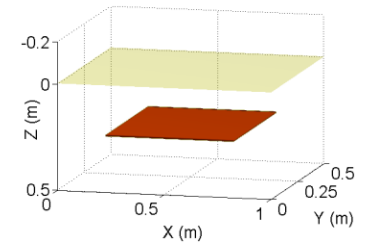

(a) Target 1: plate

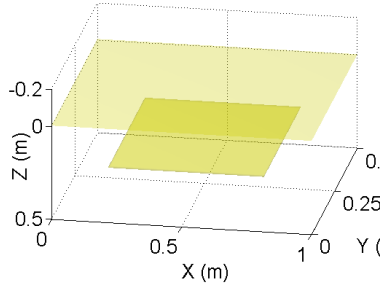

(e) Target 5: planar void

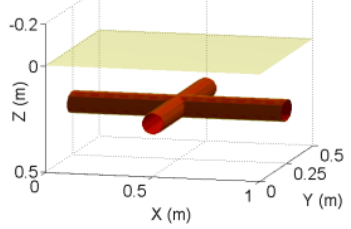

(b) Target 2: pipes

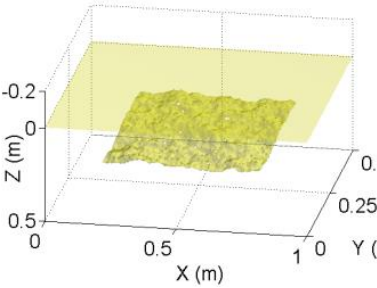

(f) Target 6: horizontal crack

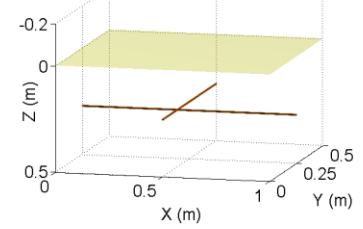

(c) Target 3: rebars

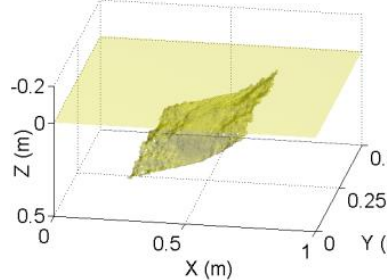

(g) Target 7: dipping crack

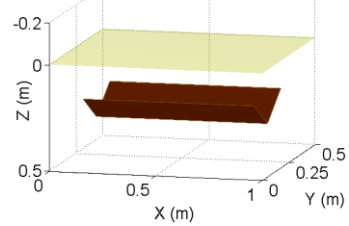

(d) Target 4: dihedral

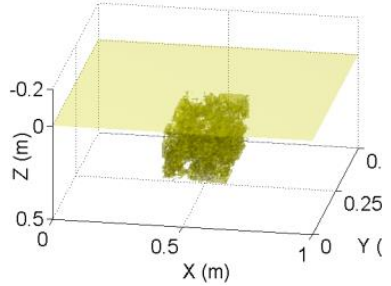

(h) Target 8: fractal volume

Fig. 5.10: Target models for simulation of polarimetric characteristic in MIMO configuration.

We employ similar sensor arrangement to the previous study described in Section 5.3. The array comprises 9 pairs of collocated transmitter and receiver with distance between each pair $7.5 \mathrm{~cm}$. This array lies over $x$-axis and moves in the direction of $y$-axis with sampling distance of $1 \mathrm{~cm}$. In SISO configuration, each pair of sensor works independently, so there is only one active pair at a particular time instance. By contrast, MIMO configuration allows these sensors to correlate. In such configuration, the transmitter is activated sequentially, while all the receivers collect the data simultaneously. We use all the entire transmitters and receivers for this MIMO configuration.

We process the data in the similar way as the previous study. The data is first preprocessed and migrated, and the polarimetric decomposition is applied afterward. Instead of repeating all the previous polarimetric decomposition methods, we focus only on 2 polarimetric techniques, namely Pauli decomposition and $\mathrm{H}$-alpha decomposition.

The simulation results for Pauli decomposition are provided in Fig. 5.11. In general, one can see that MIMO and SISO configurations produce almost similar color information regarding the shape of the targets. In agreement with the earlier results, some targets appear in different color. The plate and pipe targets yield blue color, which indicate the surface scattering in the 
Pauli component. The dihedral target exhibits red color, which is related to the double bounce scattering. The rebars target has similar characteristic as dipole, so it has the mixture between these two components and displays purple color. Although rebars and pipes seem to have almost similar size in the image, we still can distinguish their sizes by using polarimetric properties. Unfortunately, all the defect model targets appear in blue color and provide no information regarding target randomness and roughness.

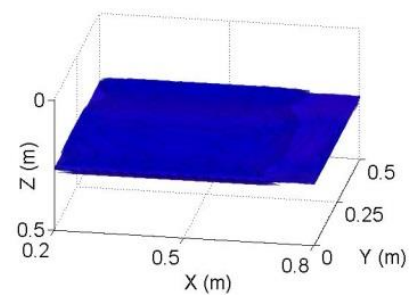

(a) Target 1 with SISO

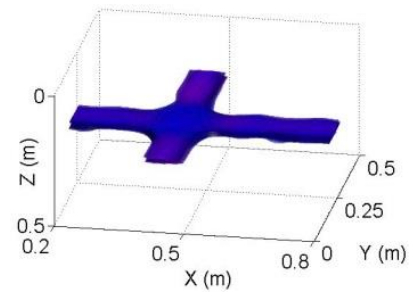

(c) Target 2 with SISO

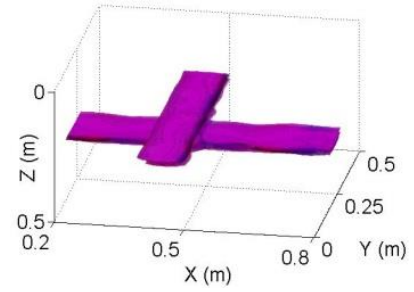

(e) Target 3 with SISO

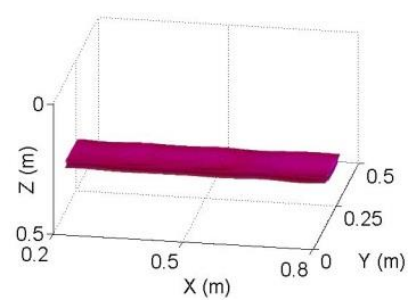

(g) Target 4 with SISO

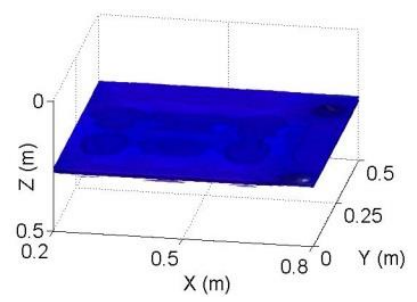

(i) Target 5 with SISO

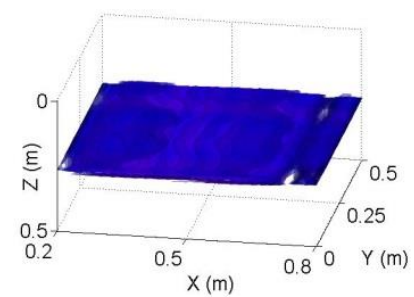

(b) Target 1 with MIMO

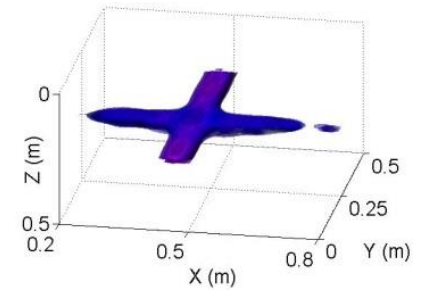

(d) Target 2 with MIMO

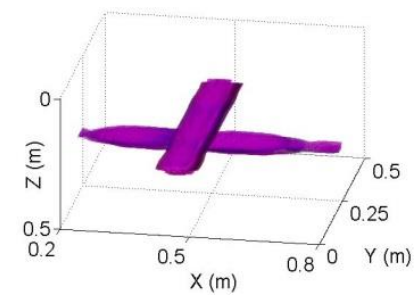

(f) Target 3 with MIMO

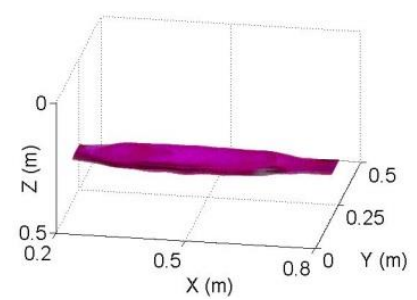

(h) Target 4 with MIMO

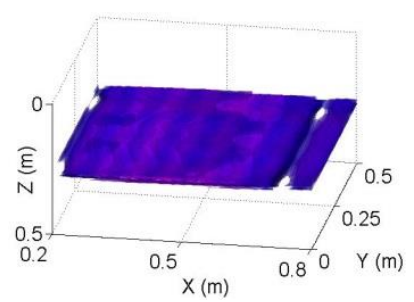

(j) Target 5 with MIMO 


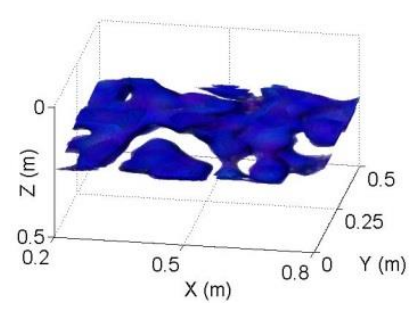

(k) Target 6 with SISO

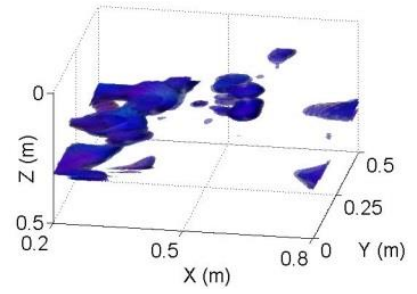

(m) Target 7 with SISO

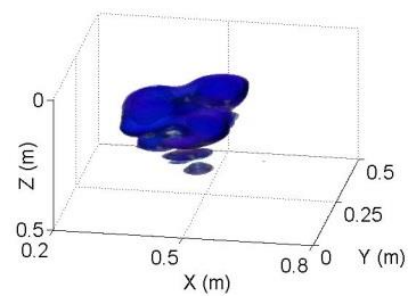

(o) Target 8 with SISO

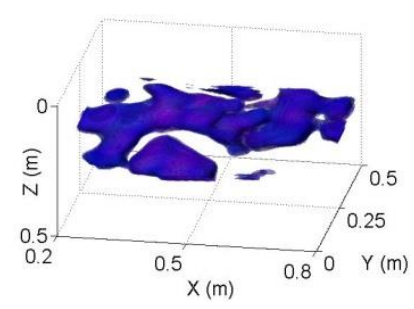

(1) Target 6 with MIMO

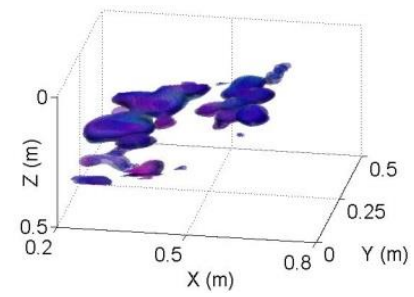

(n) Target 7 with MIMO

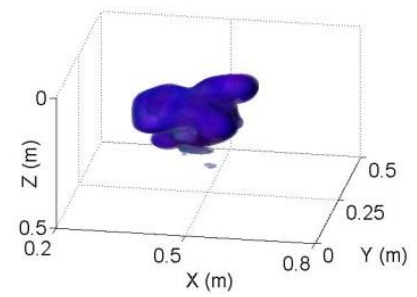

(p) Target 8 with MIMO

Fig. 5.11: Results of Pauli decomposition on SISO and MIMO configurations.

The simulation results of $\mathrm{H}$-alpha decomposition are given in Fig. 5.12. Similar to the Pauli decomposition case, MIMO and SISO configurations exhibit approximately the same behavior on their $\mathrm{H}$-alpha responses. The plate and pipes targets produce low entropy value and their alpha angle lie within $0^{\circ}$ to $30^{\circ}$. The pipes target has slightly broader distribution of alpha angle compared to the plate target. The rebars target has alpha value between $30^{\circ}$ and $60^{\circ}$, while the dihedral target produces alpha angle above $60^{\circ}$. These results are in agreement with the previous $\mathrm{H}$-alpha results in Table 5.1.

In the defect models, we can observe that the void plate has low entropy value since it has no roughness. By comparing with the $\mathrm{H}$-alpha images of PEC plate, we can see that their alpha angles are the same for SISO case. Meanwhile, the alpha angle distribution of void plate is slightly higher than PEC plate in MIMO case. The crack targets in Fig. 5.12(k) and (l) yield higher entropy value than the plate target due to its rough surface. However, the alpha value of such target remains less than $30^{\circ}$. Furthermore, the dipping crack target in Fig. 5.12(m) and (n) has higher entropy value than its horizontal version in Fig. 5.12(k) and (l). This behavior indicates that inclination angle may increase the entropy of the target. Finally, the last target in Fig. 5.12(o) and (p) provides the highest entropy among all these targets. Yet, 
this target still lies within the Bragg surface and the random surface zone in the H-alpha plane. These properties show the advantages of $\mathrm{H}$-alpha decomposition over Pauli decomposition in providing further information on target roughness and randomness by its entropy value.

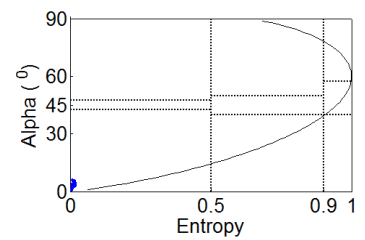

(a) Target 1 with SISO

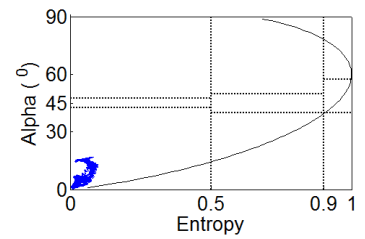

(c) Target 2 with SISO

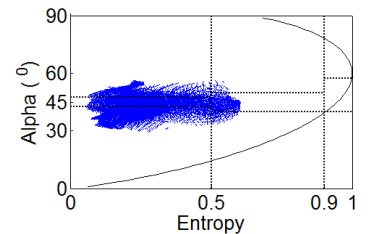

(e) Target 3 with SISO

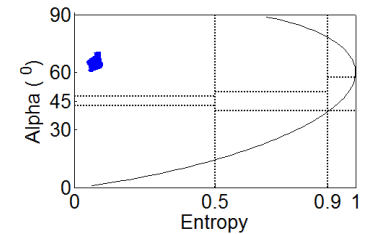

(g) Target 4 with SISO

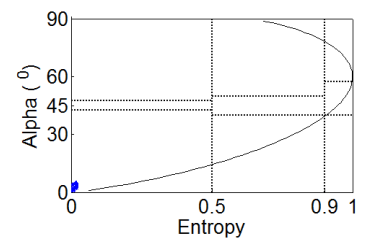

(i) Target 5 with SISO

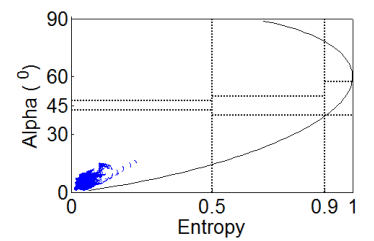

(k) Target 6 with SISO

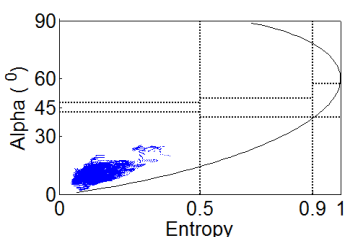

(m) Target 7 with SISO

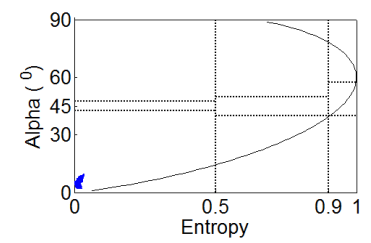

(b) Target 1 with MIMO

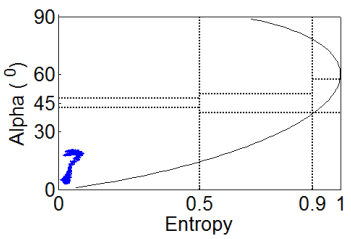

(d) Target 1 with MIMO

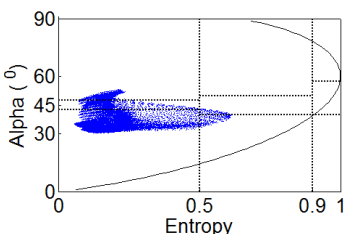

(f) Target 3 with MIMO

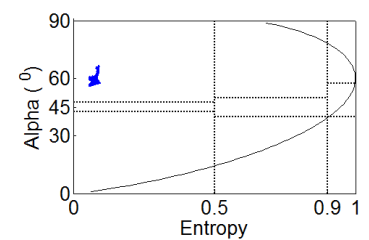

(h) Target 4 with MIMO

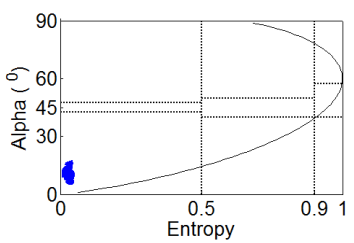

(j) Target 5 with MIMO

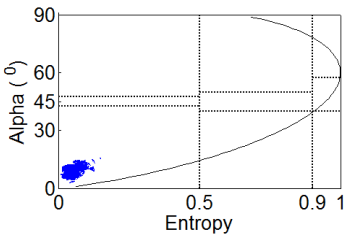

(1) Target 6 with MIMO

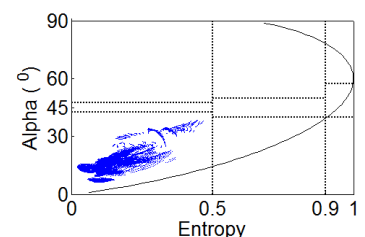

(n) Target 7 with MIMO 


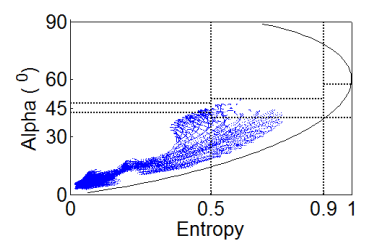

(o) Target 8 with SISO

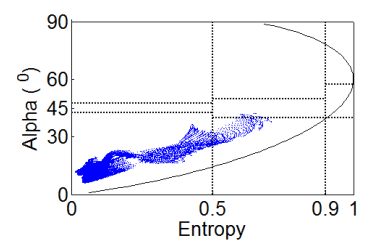

(p) Target 8 with MIMO

Fig. 5.12: Results of H-alpha decomposition on SISO and MIMO configurations.

\subsection{Concluding Remarks}

This chapter has discussed the implementation of polarization diversity on UWB GPR. The approach is by collecting the GPR data in combination of 2 linear polarizations which align with $x$ - and $y$-axes. We adopt several polarimetric decomposition techniques from PolSAR to process the fully-polarimetric GPR data. The performance of these polarimetric methods have been investigated based on simulation and experimental data.

Based on our study on simulation data, we have demonstrated the advantages of polarimetric decomposition techniques in discriminating several target shapes. Moreover, we have shown that the diameter of a cylinder target affects its polarimetric characteristic. Such dependency can be exploited to provide further information on the size of cylindrical targets, which are commonly found in utilities mapping application. These properties have been verified by the experimental data, which shows good agreement with the simulation results. In addition, the experimental data indicates the benefit of incoherent decomposition in reducing clutter components.

In the last part of our study, we compare the polarimetric characteristics of SISO and MIMO configurations based on Pauli decomposition and H-alpha decomposition. In general, we can see that MIMO and SISO configurations produce approximately similar results on these decomposition methods. Furthermore, we observe the advantages of $\mathrm{H}$-alpha decomposition over Pauli decomposition in providing information of target roughness and randomness by its entropy value. 


\section{CHAPTER 6}

\section{Conclusions and Future Work}

\subsection{Conclusions}

By the growth of infrastructure development in Singapore, inspection tools to analyze subsurface feature are highly demanded. Some existing technologies for such purpose are mechanical probe drilling, seismic tomography, and GPR. Among them, GPR is a promising solution for imaging fine geological features due to its high resolution and non-destructive properties. Nevertheless, the industrial requirements keep increasing, and the capability of current GPR system may not be adequate to fulfil such needs.

GPR system offers flexible implementation in terms of sensor configuration, transmitted waveform, and antenna polarization. These properties can potentially be exploited to improve the performance of existing GPR system. Similar concepts, which are well-known as spatial, waveform, and polarization diversities, have been used in communication and radar systems and have demonstrated various improvements on such systems.

In this thesis, all the afore-mentioned diversity aspects have been explored. The spatial diversity is implemented by introducing MIMO configuration on GPR array. The waveform diversity is applied by performing measurement in 4 different waveforms under Hermite polynomial waveform set. Finally, the polarization diversity is realized by adapting the concept of PolSAR from remote sensing field, in which the data is collected in combination of 2 orthogonal polarization bases. Throughout the chapters, these diversities are evaluated based on several simulation and experimental scenarios. Performance improvements have been demonstrated in terms of imaging capability and target characterization. In addition, several signal processing methods have been proposed to deal with the measurement modifications due to these diversities. This thesis serves a step to the development of higher performance GPR system. The key results of this thesis are summarized as follows. 


\subsubsection{Analysis on SISO, SIMO, and MIMO Imaging Performance}

In this study, MIMO configuration has been considered as an implementation of spatial diversity on UWB GPR. A comprehensive performance evaluation of SISO, SIMO, and MIMO arrays has been conducted based on simulation data.

- In comparison with SISO and SIMO arrays, MIMO configuration offers the best performance in resolution, range of dip-angle, capability in detecting small target, and SNR. Moreover, MIMO array provides reduction in side-lobe level and image artifacts. Despite of these improvements in imaging capability, MIMO configuration requires higher cost in hardware and computational complexity.

- The performance of SISO configuration is in-between SIMO and MIMO arrays. Although it cannot surpass MIMO performance, SISO array can outperform SIMO array in resolution and range of dip-angle. However, SISO configuration exhibits the highest side-lobe level among all the three arrays.

- SIMO configuration has the lowest performance in terms of resolution and side-lobe level. In addition, it suffers from imbalance reflection on particular target shapes. Yet, this array provides efficient measurement process and simple hardware implementation.

\subsubsection{Evaluation of Migration Algorithms}

Three common migration algorithms, namely Kirchhoff migration, phase-shift migration, and $\mathrm{f}-\mathrm{k}$ migration, have been evaluated for SISO and MIMO configurations.

- Based on the simulation results on both arrays, Kirchhoff migration demonstrates the best resolution compared to the other migration methods, but suffers from high sidelobe level. In addition, Kirchhoff migration has the best flexibility in sensor configuration and medium inhomogeneity at the expense of high computational complexity

- Compared to Kirchhoff migration, Phase-shift migration gives advantages in lower side-lobe level and computational complexity. However, it can handle only vertically varying medium and limited sensor configuration.

- Based on the simulation results which use homogeneous medium, f-k migration exhibits similarities with phase-shift migration in terms of resolution and side-lobe 
level. Although this method offers the best efficiency in computational complexity among the three algorithms, it is applicable only on limited sensor configuration. Moreover, the imaging result of this migration method degrades in inhomogeneous medium case.

- An equivalent phase-shift migration on SIMO configuration has been proposed. Originally, phase-shift migration and $\mathrm{f}-\mathrm{k}$ migration have limitation in sensor configuration and are unable to handle SIMO configuration. By modifying the scattered wave equation on SIMO array, we propose a migration method that has equivalent computation steps with phase-shift migration.

\subsubsection{Efficient Autofocusing Method for MIMO Configuration}

Wave velocity is necessary information in GPR signal processing in order to obtain highly focused image. However, this information is unknown in practice and needs to be estimated from the data. Autofocusing methods are available for velocity estimation on SISO configuration, but the computation becomes tedious for MIMO configuration. To resolve this issue, an efficient autofocusing method for MIMO array has been proposed.

- The autofocusing method utilizes MZO operator that can transform MIMO data into its equivalent SISO data, and thus reduce the computation burden.

- This autofocusing method estimates the wave velocity directly from the measurement data, where image entropy is used as the focusing parameter. Therefore, no additional measurement is needed.

- Performance study has been conducted to compare this autofocusing method with the standard autofocusing methods on SISO and MIMO arrays. The proposed autofocusing method successfully demonstrates significant reduction in computation time while preserving the imaging capability of MIMO configuration.

\subsubsection{Implementation of Waveform Diversity on UWB GPR}

Waveform diversity method has been proposed for UWB GPR.

- The waveform diversity method is implemented by collecting the GPR data with the first four order of Hermite polynomial waveform set.

- A processing method has been developed to combine these datasets into a single image and to extract more information regarding the target. Simulation has been 
conducted to verify this method, and the results indicate that the proposed method can perform binary classification on targets made of void, water, and PEC. Moreover, waveform diversity can also improve the resolution and range of dip-angle of the image.

\subsubsection{Evaluation of Polarimetric Decomposition Methods on UWB GPR for Utilities Mapping Application}

Several polarimetric decomposition methods from PolSAR have been applied and evaluated on UWB GPR data with focus on utilities mapping application.

- Simulation data and experimental data have been generated on several target models that are common in utilities mapping application.

- The results show that most of the incoherent and coherent decomposition methods are able to provide information on the target shape. In addition, these decomposition methods also exhibit different responses on cylindrical targets with various diameters. Such properties are advantageous in providing a rough estimation on target size. However, due to the utilization of second order statistics, incoherent decompositions can reduce the noisy components in the image, while coherent decomposition suffers from higher image artifacts and clutter.

- H-alpha decomposition can provide quantitative information of target shapes and randomness. The alpha angle changes continuously with respect to target shape, from planar target, dipole target, to dihedral target. Meanwhile, the entropy value indicates the amount of target roughness and randomness.

- MIMO and SISO configurations show almost similar polarimetric characteristic. Nevertheless, the imaging capability of MIMO array and the target characterization properties of fully-polarimetric measurement are promising to be combined for future study.

\subsection{Future Work}

In this section, we provide several future works of this research as follows:

1) Method to combine spatial, waveform, and polarization diversities. In this research, we have analyzed the capabilities of spatial, waveform, and polarization diversities 
independently. Each method has shown its own advantages and disadvantages. The combination of these methods can potentially improve the imaging performance and still needs further exploration.

2) Optimization on polarimetric decomposition for GPR data. The polarimetric decomposition methods that have been evaluated so far are obtained from PolSAR. Since these methods are originally developed for remote sensing application, such as terrain, land-used, vegetation, ocean, and ice classifications, they may not provide suitable information on GPR target. Polarimetric decomposition methods that are specifically designed for GPR target is still an open topic for future research.

3) Automatic target recognition algorithm. We have investigated and developed target characterization method based on waveform and polarization diversities information. However, some target identifications are still carried out manually based on color information and plotting diagram. An automatic target recognition algorithm needs to be developed in the future.

4) Implementation on other applications of UWB imaging. In this study, we provide the implementation of spatial, waveform, and polarization diversities on UWB GPR. In fact, there are various UWB imaging systems that carry almost similar principle to UWB GPR. The methods proposed in this research can possibly be modified and applied to other UWB imaging systems. 


\section{AUTHOR'S PUBLICATIONS}

\section{JOURNAL PAPERS:}

[1] D. H. N. Marpaung and Y. L. Lu, "Autofocusing of UWB MIMO GPR images by using MZO and entropy minimization," IEEE Geoscience and Remote Sensing Letters, vol. 13, no. 5, pp. 661-665, May 2016.

[2] D. H. N. Marpaung and Y. L. Lu, "A comparison study of SISO, SIMO, and MIMO configurations for GPR imaging," submitted to IEEE Transactions on Geoscience and Remote Sensing, 2017.

[3] D. H. N. Marpaung and Y. L. Lu, "A study of polarimetry decompositions on UWB GPR applications," submitted to IET Radar Sonar \& Navigation, 2017.

\section{CONFERENCE PAPERS}

[1] D. H. N. Marpaung and Y. L. Lu, "A comparative study of migration algorithms for UWB GPR images in SISO-SAR and MIMO-array configurations," Proc. 15th International Radar Symposium (IRS) 2014, pp. 1-4, 16-18 Jun. 2014.

[2] D. H. N. Marpaung and Y. L. Lu, "Investigation of MIMO and SAR performance on several applications of UWB GPR," Proc. 2014 IEEE International Conference on Aerospace Electronics and Remote Sensing Technology (ICARES 2014), pp. 196-199, 13-14 Nov. 2014.

[3] D. H. N. Marpaung and Y. L. Lu, "Waveform diversity for ultra-wideband ground penetrating radar imaging," presented in Proc. $36^{\text {th }}$ Progress in Electromagnetics Research Symposium, 6-9 Jul. 2015, Prague, Czech.

[4] D. H. N. Marpaung and Y. L. Lu, "Improved void detection by using MIMO SAR ground penetrating radar," presented in Proc. $5^{\text {th }}$ Asia-Pacific Conference on Synthetic Aperture Radar, 2-4 Sep. 2015, Singapore. 
[5] D. H. N. Marpaung and Y. L. Lu, "A study of polarimetric UWB GPR imaging for utility mapping survey applications," Proc. $16^{\text {th }}$ International Conference on Ground Penetrating Radar, pp., 13-16 Jun. 2016. (Shortlisted for Young Scientist Awards) 


\section{BIBLIOGRAPHY}

[1] S.V.D. Lee, "Seismic Tomography," Earthscope Workshop, Monterey CA, 2007.

[2] J. Sheng, "High Resolution Seismic Tomography with the Generalized Randon Transform and Early Arrival Waveform Inversion," PhD Dissertation, The University of Utah, 2004.

[3] J.P. Mooney, J.D. Ciampa, G.N. Young, A.R. Kressner, and J. Carbonara, "GPR mapping to avoid utility conflicts prior to construction of the M-29 transmission line," Proc of IEEE PES T\&D 2010, pp. 1-8, 2010.

[4] A. Zarkhidze and E. Lemenager, "Case study - use of 3D GPR technologies for utility mapping in paris," Proc of the 10th International Conference on Ground Penetrating Radar, pp. 375-378, 2004.

[5] F.M. Fernandes and J. Pais, "Assessment of moisture in road pavements," Proc of 15th International Conference on Ground Penetrating Radar, pp. 909-912, 2014.

[6] C. Ékes, P. Takacs, and B. Neducza, "Condition assessment of critical infrastructure with GPR," Proc of 15th International Conference on Ground Penetrating Radar, pp. 429-434, 2014.

[7] A. Benedetto, F. Tosti, G. Schettini, and C. Twizere, "Evaluation of geotechnical stability of road using GPR," Proc of 6th International Workshop on Advanced Ground Penetrating Radar, pp. 1-6, 2011.

[8] A. Simi, G. Manacorda, and A. Benedetto, "Bridge deck survey with high resolution Ground Penetrating Radar," Proc of 14th International Conference on Ground Penetrating Radar, pp. 489-495, 2012.

[9] P. Kaur, K.J. Dana, F.A. Romero, and N. Gucunski, "Automated GPR rebar analysis for robotic bridge deck evaluation," IEEE Transactions on Cybernetics, vol. PP, no. 99, pp. 1-12, Oct. 2015.

[10] E. Eide, P.A. Sandnes, B. Nilssen, and S. Tjora, "Airfield runway inspection using 3 dimensional GPR," Proc of the 3rd International Workshop on Advanced Ground Penetrating Radar, pp. 87-91, 2005.

[11] W. Al-Nuaimy, A. Eriksen, and J. Gasgoyne, "Train-mounted GPR for high-speed rail trackbed inspection," Proc of the Tenth International Conference on Ground 
Penetrating Radar, pp. 631-634, 2004.

[12] H. Liu, C. N. Koyama, K. Takahashi, and M. Sato, "High-resolution imaging of damaged wooden structures for building inspection by polarimetric radar," Proc of 15th International Conference on Ground Penetrating Radar, pp. 423-428, 2014.

[13] C.N. Koyama, K. Takahashi, Y. Iitsuka, and M. Sato, "Polarimetric UWB SAR for subsurface imaging of building structures," Proc of 2014 Asia-Pacific Microwave Conference, pp. 1025-1027, 2014.

[14] D. Arosio, S. Munda, and L. Zanzi, "A case study where dual-polarization was essential for correct interpretation of GPR results," Proc of 15th International Conference on Ground Penetrating Radar, pp. 8-12, 2014.

[15] A. Valls, F. García, M. Ramírez, and J. Benlloch, "A combined use of GPR data with historical archives for identifying pavement construction periods of Valencian Silos (16th Century, Spain)," IEEE Journal of Selected Topics in Applied Earth Observations and Remote Sensing, vol. 9, no. 1, pp. 98-107, Jan. 2016.

[16] M. Sato, H. Liu, T. Komagino, and K. Takahashi, "Archaeological survey by GPR for recovery from 3.11 great earthquake and tsunami in East Japan," Proc of 15th International Conference on Ground Penetrating Radar, pp. 25-30, 2014.

[17] T.G. Savelyev, L. van Kempen, H. Sahli, J. Sachs, and M. Sato, "Investigation of timefrequency features for GPR landmine discrimination," IEEE Transactions on Geoscience and Remote Sensing, vol. 45, no. 1, pp. 118-129, Jan. 2007.

[18] X. Feng, M. Sato, Y. Zhang, C. Liu, F. Shi, and Y. Zhao, "CMP antenna array GPR and signal-to-clutter ratio improvement," IEEE Geoscience and Remote Sensing Letters, vol. 6, no. 1, pp. 23-27, Jan. 2009.

[19] "International Campaign to Ban Landmines," [Online]. Available: http://www.icbl.org.

[20] X. Feng, M. Sato, and C. Liu, "Subsurface imaging using a handheld GPR MD system," IEEE Geoscience and Remote Sensing Letters, vol. 9, no. 4, pp. 659-662, Jul. 2012.

[21] A.G. Yarovoy, T.G. Savelyev, P.J. Aubry, P.E. Lys, and L.P. Ligthart, "UWB Arraybased sensor for near-field imaging," IEEE Transactions on Microwave Theory and Techniques, vol. 55, no. 6, pp. 1288-1295, Jun. 2007.

[22] C.-C. Chen, M.B. Higgins, K. O'Neill, and R. Detsch, "Ultrawide-bandwidth fully- 
polarimetric ground penetrating radar classification of subsurface unexploded ordnance," IEEE Transactions on Geoscience and Remote Sensing, vol. 39, no. 6, pp. 1221-1230, Jun. 2001.

[23] R. Greaves, D. Lesmes, J. Lee, and M. Toksov, "Velocity variations and water content estimated from multi-offset, ground-penetrating radar," Geophysics, vol. 61, pp. 683695, Jun. 1996.

[24] A. Chanzy, A. Tarussov, A. Judge, and F. Bonn, "Soil water content determination using digital ground penetrating radar," Soil Sci. Soc. Amer. J., vol. 60, pp. 1318-1326, 1996.

[25] R. van Overmeeren, S. Sariowan, and J. Gehrels, "Ground penetrating radar for determining volumetric soil water content: Results of comparative measurements at two test sites," J. Hydrol., vol. 197, pp. 316-338, Oct. 1997.

[26] K.Weiler, T. Steenhuis, J. Boll, and K.-J. Kung, "Comparison of ground penetrating radar and time domain reflectometry as soil water sensors," Soil Sci. Soc. Amer. J., vol. 62, pp. 1237-1239, 1998.

[27] J. Huisman, C. Sperl, W. Bouten, and J. Verstraten, "Soil water content measurements at different scales: accuracy of time domain reflectometry and ground penetrating radar," J. Hydrol., vol. 245, pp. 48-58, May 2001.

[28] G. Serbin and D. Or, "Near-surface water content measurements using horn antenna radar: methodology and overview," Vadose Zone J., vol. 2, pp. 500-510, 2003.

[29] S. Lambot, E. Slob, I. van den Bosch, B. Stockbroeckx, B. Scheers, and M. Vanclooster, "Estimating soil electric properties from monostatic ground-penetrating radar signal inversion in the frequency domain," Water Resources Res., vol. 40, pp. W04 205, Apr. 2004.

[30] S. Lambot, E.C. Slob, I. van den Bosch, B. Stockbroeckx, B. Scheers, and M. Vanclooster, "GPR design and modeling for identifying the shallow subsurface dielectric properties," Proc. 2nd Int. Workshop Advanced Ground Penetrating Radar, pp. 130-135, 2003.

[31] S. Lambot, E.C. Slob, I. van den Bosch, B. Stockbroeckx, and M. Vanclooster, "Modeling of ground-penetrating Radar for accurate characterization of subsurface electric properties," IEEE Transactions on Geoscience and Remote Sensing, vol. 42, no. 11, pp. 2555-2568, Nov. 2004. 
[32] S. Hubbard, Y. Rubin, and E. Majer, "Ground-penetrating-radar-assisted saturation and permeability estimation in bimodal systems," Water Resources Res., vol. 33, pp. 971990, May 1997.

[33] E. Gloaguen, M. Couteau, D. Marcotte, and R. Chapuis, "Estimation of hydraulic conductivity of an unconfined aquifer using cokriging of GPR and hydrostratigraphic data," J. Appl. Geophys., vol. 47, p. 135-152, Jun. 2001.

[34] K. Langley et al., "From glacier facies to SAR backscatter zones via GPR," IEEE Transactions on Geoscience and Remote Sensing, vol. 46, no. 9, pp. 2506-2516, Sept. 2008.

[35] S. Hamran and K. Langley, "A $5.3 \mathrm{GHz}$ step-frequency GPR for glacier surface characterisation," Proc of the Tenth International Conference on Ground Penetrating Radar, pp. 761-764, 2004.

[36] R. Qian and L. Liu, "Internal structure of sand dunes in the Badain Jaran Desert revealed by GPR," IEEE Journal of Selected Topics in Applied Earth Observations and Remote Sensing, vol. 9, no. 1, pp. 159-166, Jan. 2016.

[37] P. M. Barone et al., "Ground-penetrating radar technique to investigate historic eruptions on the Mt. Etna volcano (Sicily, Italy)," Proc of 7th International Workshop on Advanced Ground Penetrating Radar, pp. 1-6, 2013.

[38] J. D. Taylor, Introduction to Ultra-Wideband Radar Systems, Boca Raton, USA: CRC Press, 1995.

[39] L. Carin, N. Geng, M. McClure, J. Sichina, and L. Nguyen, "Ultra-wide-band synthetic-aperture radar for mine-field detection," IEEE Antennas and Propagation Magazine, vol. 41, no. 1, pp. 18-33, Feb. 1999.

[40] A.G. Yarovoy, L.P. Ligthart, J. Matuzas, and B. Levitas, "UWB radar for human being detection," IEEE Aerospace and Electronic Systems Magazine, vol. 21, no. 3, pp. 2226, Nov. 2006.

[41] X. Li, E.J. Bond, B.D.V. Veen, and S.C. Hagness, "An overview of ultra-wideband microwave imaging via space-time beamforming for early-stage breast-cancer detection," IEEE Antennas and Propagation Magazine, vol. 47, no. 1, pp. 19-34, Feb. 2005.

[42] S. Gezici et al., "Localization via ultra-wideband radios: a look at positioning aspects for future sensor networks," IEEE Signal Processing Magazine, vol. 22, no. 4, pp. 70- 
84, Jul. 2005.

[43] L. Yang and G.B. Giannakis, "Ultra-wideband communications: an idea whose time has come," IEEE Signal Processing Magazine, vol. 21, no. 6, pp. 26-54, Nov. 2004.

[44] G.R. Aiello and G.D. Rogerson, "Ultra-wideband wireless systems," IEEE Microwave Magazine, vol. 4, no. 2, pp. 36-47, Jun. 2003.

[45] D. Porcino and W. Hirt, "Ultra-wideband radio technology: potential and challenges ahead," IEEE Communications Magazine, vol. 41, no. 7, pp. 66-74, Jul. 2003.

[46] D. D. Wentzloff, R. Blazquez, F.S. Lee, B.P. Ginsburg, J. Powell, and A.P. Chandrakasan, "System design considerations for ultra-wideband communication," IEEE Communications Magazine, vol. 43, no. 8, pp. 114-121, Aug. 2005.

[47] R.C. Qiu, H. Liu, and X. Shen, "Ultra-wideband for multiple access communications," IEEE Communications Magazine, vol. 43, no. 2, pp. 80-87, Feb. 2005.

[48] P.A. Catherwood and W.G. Scanlon, "Ultrawideband communications - an idea whose time has still yet to come? [Wireless Corner]," IEEE Antennas and Propagation Magazine, vol. 57, no. 2, pp. 38-43, Apr. 2015.

[49] N. Diamanti, D. Redman, and A. Giannopoulos, "A study of GPR vertical crack responses in pavement using field data and numerical modelling," Proc of 13th International Conference on Ground Penetrating Radar, pp. 1-6, 2010.

[50] G. Roqueta, L. Jofre, and M.Q. Feng, "Analysis of the electromagnetic signature of reinforced concrete structures for nondestructive evaluation of corrosion damage," IEEE Transactions on Instrumentation and Measurement, vol. 61, no. 4, pp. 10901098, Apr. 2012.

[51] M. Sato, K. Takahashi, H. Liu, and C. Koyama, "Investigation on near range SAR for inspecting inner structure of buildings," Proc of 2014 IEEE Geoscience and Remote Sensing Symposium, pp. 2601-2604, 2014.

[52] C.N. Koyama, Y. Iitsuka, K. Takahashi, and M. Sato, "Full polarimetric UWB GBSAR for damage assessment of wooden building structures," Proc of 2015 IEEE International Geoscience and Remote Sensing Symposium, pp. 2719-2722, 2015.

[53] X. Feng, M. Sato, and C. Liu, "Hand-held GPR imaging using migration for irregular data," IEEE Journal of Selected Topics in Applied Earth Observations and Remote Sensing, vol. 4, no. 4, pp. 799-803, Dec. 2011. 
[54] A. Yarovoy et al., "Performance of UWB array-based radar sensor in a multi-sensor vehicle-based suit for landmine detection," Proc of EuRAD 2008, pp. 288-291, 2008.

[55] T. Counts, A.C. Gurbuz, W.R. Scott, J.H. McClellan, and K. Kim, "Multistatic groundpenetrating radar experiments," IEEE Transactions on Geoscience and Remote Sensing, vol. 45, no. 8, pp. 2544-2553, Aug. 2007.

[56] T. Jin, J. Lou, and Z. Zhou, "Extraction of landmine features using a forward-looking ground-penetrating radar with MIMO array," IEEE Transactions on Geoscience and Remote Sensing, vol. 50, no. 10, pp. 4135-4144, Oct. 2012.

[57] D.W. Paglieroni, D.H. Chambers, J.E. Mast, S.W. Bond, and N.R. Beer, "Imaging modes for ground penetrating radar and their relation to detection performance," IEEE Journal of Selected Topics in Applied Earth Observations and Remote Sensing, vol. 8, no. 3, pp. 1132-1144, Mar. 2015.

[58] A.M. Haimovich, R.S. Blum, and L.J. Cimini, "MIMO radar with widely separated antennas," IEEE Signal Processing Magazine, vol. 25, no. 1, pp. 116-129, 2008.

[59] J. Li and P. Stoica, "MIMO radar with colocated antennas," IEEE Signal Processing Magazine, vol. 24, no. 5, pp. 106-114, Sept. 2007.

[60] X. Zhuge and A.G. Yarovoy, "Sparse multiple-input multiple-output arrays for highresolution near-field ultra-wideband imaging," IET Microwaves, Antennas \& Propagation, vol. 5, no. 13, pp. 1552-1562, Oct. 2011.

[61] X. Zhuge and A.G. Yarovoy, "A sparse aperture MIMO-SAR-based UWB imaging system for concealed weapon detection," IEEE Transactions on Geoscience and Remote Sensing, vol. 49, no. 1, pp. 509-518, Jan. 2011.

[62] A.M. Guidi, T.A. Sturman, P. Dingley, M.D.J. Bowyer, N.R. Petfield, and M. Moseley, "Waveform diversity and design: Part I - Issues for multi-antenna systems," Proc of 2008 IET Waveform Diversity \& Digital Radar Conference, pp. 1-11, 2008.

[63] T.A. Sturman, A.M. Guidi, P. Dingley, M.D.J. Bowyer, N.R. Petfield, and M. Moseley, "Waveform diversity and design: Part II - Issues for waveforms and applications," Proc of 2008 IET Waveform Diversity \& Digital Radar Conference, pp. 1-13, 2008.

[64] M. Vespe, G. Jones, and C.J. Baker, "Lessons for radar," IEEE Signal Processing Magazine, vol. 26, no. 1, pp. 65-75, Jan. 2009.

[65] J. Wang, Y. Qin, H. Wang, and X. Li, "Dynamic waveform selection for manoeuvering 
target tracking in clutter," IET Radar, Sonar \& Navigation, vol. 7, no. 7, pp. 815-825, Aug. 2013.

[66] W.Q. Wang, "Large time-bandwidth product MIMO radar waveform design based on chirp rate diversity," IEEE Sensors Journal, vol. 15, no. 2, pp. 1027-1034, Feb. 2015.

[67] W.Q. Wang, Q. Peng, and C. Jingye, "Waveform-diversity-based millimeter-wave UAV SAR remote sensing," IEEE Trans. on Geoscience and Remote Sensing, vol. 47, no. 3, pp. 691-700, Mar. 2009.

[68] W.Q. Wang and H. Shao, "Two-antenna SAR with waveform diversity for ground moving target indication," IEEE Geoscience and Remote Sensing Letters, vol. 11, no. 12, pp. 2154-2158, Dec. 2014.

[69] A.G. Yarovoy, L.P. Ligthart, A.D. Schukin, and I.V. Kaploun, "Multi-waveform fullpolarimetric GPR for landmine detection," Proc of 2003 IEEE International Geoscience and Remote Sensing Symposium, pp. 749-751, 2003.

[70] B. Hu and N.C. Beaulieu, "Pulse shapes for ultrawideband communication systems," IEEE Trans. on Wireless Communications, vol. 4, no. 4, pp. 1789-1797, Jul. 2005.

[71] X. Feng et al., "Application of freeman decomposition to full polarimetric GPR," Proc of 2013 IEEE Int. Geoscience and Remote Sensing Symposium, pp. 3534-3537, 2013.

[72] X. Feng, Y. Yu, C. Liu, and M. Fehler, "Combination of H-Alpha decomposition and migration for enhancing subsurface target classification of GPR," IEEE Trans. on Geoscience and Remote Sensing, vol. 53, no. 9, pp. 4852-4861, Sept. 2015.

[73] T. Miwa, M. Sato, and H. Niitsuma, "Subsurface fracture measurement with polarimetric borehole radar," IEEE Transactions on Geoscience and Remote Sensing, vol. 37, no. 2, pp. 828-837, Mar. 1999.

[74] J.G. Zhao and M. Sato, "Radar polarimetry analysis applied to single-hole fully polarimetric borehole radar," IEEE Transactions on Geoscience and Remote Sensing, vol. 44, no. 12, pp. 3547-3554, Dec. 2006.

[75] J.G. Zhao and M. Sato, "Consistency analysis of subsurface fracture characterization using different polarimetry techniques by a borehole radar," IEEE Geoscience and Remote Sensing Letters, vol. 4, no. 3, pp. 359-363, Jul. 2007.

[76] "Designing Our City - Planning for a Sustainable Singapore," Urban Redevelopment Authority, Jul. 2012. 
[77] P.C. Lui, J. Zhao, and Y.X. Zhou "Creation of space in rock caverns in Singapore past, present and future," in Y.X. Zhou, J.G. Cai, and R. Sterling (eds), Advances in Underground Space Development, Research Publishing, 2012.

[78] T. Misir, "Singapore looks underground for room to grow," Citiscope, 28 May 2015.

[79] J.F. Hjelmstad and E.S. Eide, "3-D imaging using novel techniques in ultra-wideband radar," Proc of 30th European Microwave Conference, pp. 1-4, 2000.

[80] F. Elbahhar, A. Rivenq-Menhaj, J.M. Rouvaen, M. Heddebaut, and T. Boukour, "Comparison between DS-CDMA and modified Gegenbauer functions for a multiuser communication ultra-wideband system," IEE Proceedings - Communications, vol. 152, no. 6, pp. 1021-1027, Dec. 2005.

[81] A.S. Turk, A.K. Hocaoglu, and A.A. Vertiy, Subsurface Sensing, Hoboken, New Jersey: John Wiley \& Sons, Inc., 2011.

[82] D.J. Daniels, Ground Penetrating Radar - 2nd Edition, London, United Kingdom: The Institution of Electrical Engineers, 2004.

[83] D. Ghosh and T.K. Sarkar, "Extraction of the signature of a buried object using GPR," Proc of 2006 IEEE Conference on Radar, pp. 296-301, 2006.

[84] B. Karlsen, J. Larsen, K.B. Jakobsen, H.B. Sorensen, and S. Abrahamson, "Antenna characteristics and air-ground interface de-embedding methods for stepped-frequency ground-penetrating radar measurements," Proc. SPIE 4038, Detection and Remediation Technologies for Mines and Minelike Targets V, 2000.

[85] A. Bennia and S.M. Riad, "An optimization technique for iterative frequency-domain deconvolution," IEEE Transactions on Instrumentation and Measurement, vol. 39, no. 2, pp. 358-362, Apr. 1990.

[86] A. Giannopoulos, "Modelling ground penetrating radar by GprMax," Construction and Building Materials, vol. 19, no. 10, pp. 755-762, Dec. 2005.

[87] R. Yelf, "Where is true time zero?," Proc of the Tenth International Conference on Ground Penetrating Radar, pp. 279-282, 2004.

[88] P. Gader, W.-H. Lee, and J.N. Wilson, "Detecting landmines with ground-penetrating radar using feature-based rules, order statistics, and adaptive whitening," IEEE Transactions on Geoscience and Remote Sensing, vol. 42, no. 11, pp. 2522-2534, Nov. 2004. 
[89] S. Tjora, E. Eide, and L. Lundheim, "Evaluation of methods for ground bounce removal in GPR utility mapping," Proc of the Tenth International Conference on Ground Penetrating Radar, pp. 379-382, 2004.

[90] B. Karlsen, J. Larsen, H.B.D. Sorensen, and K.B. Jakobsen, "Comparison of PCA and ICA based clutter reduction in GPR systems for anti-personal landmine detection," Proc of the 11th IEEE Signal Processing Workshop on Statistical Signal Processing, pp. 146-149, 2001.

[91] W.A. Schneider, "Integral formulation for migration in two and three dimensions," Geophysics, vol. 43, pp. 49-76, Feb. 1978.

[92] R.H. Stolt, "Migration by Fourier transform," Geophysics, vol. 43 (1), pp. 23-48, Feb. 1978.

[93] J. Gazdag, "Wave equation migration with the phase shift method," Geophysics, vol. 43, pp. 1342-1351, Dec. 1978.

[94] X. Lu and H. Sun, "Parameter assessment for SAR image quality evaluation system," Proc of 1st Asian and Pacific Conference on Synthetic Aperture Radar, pp. 58-60, 2007.

[95] R.H. Stolt and A.B. Weglein, Seismic Imaging and Inversion - Application of Linear Inverse Theory, New York, United States of America: Cambridge University Press, 2012.

[96] N. Bleistein, J.K. Cohen, and J.W. Stockwell Jr., Mathematics of Multidimensional Seismic Imaging, Migration, and Inversion, New York: Springer, 2001.

[97] M.T. Taner and F. Koehler, "Velocity spectra-digital computer derivation and applications of velocity functions," Geophysics, vol. 34, no. 6, pp. 859-881, Dec. 1969.

[98] C.H. Dix, "Seismic velocities from surface measurements," Geophysics, vol. 20, no. 1, pp. 68-86, Jan. 1955.

[99] Y.L. Liu, L.L. Li, and F. Li, "Imaging of two-dimensional targets buried in a lossy earth with unknown characteristics from multi-frequency and multi-monostatic data," IET Microwaves, Antennas \& Propagation, vol. 4, no. 10, pp. 1647-1653, Oct. 2010.

[100] L. Li, W. Zhang, and F. Li, "A novel autofocusing approach for real-time through-wall imaging under unknown wall characteristics," IEEE Transactions on Geoscience and Remote Sensing, vol. 48, no. 1, pp. 423-431, Jan. 2010. 
[101] X. Xu, E.L. Miller, and C.M. Rappaport, "Minimum entropy regularization in frequency-wavenumber migration to localize subsurface objects," IEEE Transactions on Geoscience and Remote Sensing, vol. 41, no. 8, pp. 1804-1812, Aug. 2003.

[102] X. Wei and Y. Zhang, "Autofocusing techniques for GPR data from RC bridge decks," IEEE Journal of Selected Topics in Applied Earth Observations and Remote Sensing, vol. 7, no. 12, pp. 4860-4868, Dec. 2014.

[103] A.J. Devaney, Mathematical Foundations of Imaging, Tomography and Wavefield Inversion, New York: Cambridge University Press, 2012.

[104] X. Zhuge, T.G. Savelyev, A.G. Yarovoy, and L.P. Ligthart, "UWB array-based radar imaging using modified Kirchhoff migration," Proc of 2008 International Conference on Ultra-Wideband, pp. 175-178, 2008.

[105] X. Zhuge, T.G. Savelyev, A.G. Yarovoy, L.P. Ligthart, and B. Levitas, "Comparison of different migration techniques for UWB short-range imaging," Proc of EuRAD 2009, pp. 184-187, 2009.

[106] X. Zhuge and A. Yarovoy, "Near-field ultra-wideband imaging with two-dimensional sparse MIMO array," Proc of the 4th European Conference on Antennas and Propagation, pp.1-4, 2010.

[107] I.D. Hale, "Dip-moveout by Fourier Transform," PhD Dissertation, Stanford University, 1983.

[108] M. Balsi et al., "FDTD simulation of GPR measurements in a laboratory sandbox for landmine detection," Proc of 2009 5th Int. Workshop of Advanced GPR, pp. 45-49, 2009.

[109] D.J. Kershaw and R.J. Evans, "Optimal waveform selection for tracking systems," IEEE Trans. on Information Theory, vol. 40, no. 5, p. 1536-1550, Sept. 1994.

[110] L. Sakkila, A. Rivenq, C. Tatkeu, Y. ElHillali, F. Boukour, and J.M. Rouvaen, "Comparison of classical and orthogonal UWB waveforms for radar applications," Proc of 2010 6th International Colloquium on Signal Processing and Its Applications, pp. 1-5, 2010.

[111] S. Haykin, Neural Networks A Comprehensive Foundation 2nd Edition, India: Pearson Education Inc., 2005.

[112] E. Pasolli, F. Melgani, and M. Donelli, "Automatic analysis of GPR images: a pattern- 
recognition approach," IEEE Transactions on Geoscience and Remote Sensing, vol. 47, no. 7, pp. 2206-2217, Jul. 2009.

[113] W. Shao, A. Bouzerdoum, S.L. Phung, L. Su, B. Indraratna, and C. Rujikiatkamjorn, "Automatic classification of GPR signals," Proc of 2010 13th International Conference on Ground Penetrating Radar, pp. 1-6, 2010.

[114] H. Zhang, S. Ouyang, G. Wang, S. Wu, and F. Zhang, "Dielectric spectrum feature vector extraction algorithm of ground penetrating radar signal in frequency bands," IEEE Geoscience and Remote Sensing Letters, vol. 12, no. 5, pp. 958-962, May 2015.

[115] M.S. El-Mahallawy and M. Hashim, "Material classification of underground utilities from GPR images using DCT-based SVM approach," IEEE Geoscience and Remote Sensing Letters, vol. 10, no. 6, pp. 1542-1546, Nov. 2013.

[116] S. Caorsi and G. Cevini, "An electromagnetic approach based on neural networks for the GPR investigation of buried cylinders," IEEE Geoscience and Remote Sensing Letters, vol. 2, no. 1, pp. 3-7, Jan. 2005.

[117] E. Rignot, R. Chellappa, and P. Dubois, "Unsupervised segmentation of polarimetric SAR data using the covariance matrix," IEEE Transactions on Geoscience and Remote Sensing, vol. 30, no. 4, pp. 697-705, Jul. 1992.

[118] K.S. Chen, W.P. Huang, D.H. Tsay, and F. Amar, "Classification of multifrequency polarimetric SAR imagery using a dynamic learning neural network," IEEE Transactions on Geoscience and Remote Sensing, vol. 34, no. 3, pp. 814-820, May 1996.

[119] Y.C. Tzeng and K.S. Chen, "A fuzzy neural network to SAR image classification," IEEE Transactions on Geoscience and Remote Sensing, vol. 36, no. 1, pp. 301-307, Jan. 1998.

[120] L.F. Famil, E. Pottier, and J.S. Lee, "Unsupervised classification of multifrequency and fully polarimetric SAR images based on the H/A/Alpha-Wishart classifier," IEEE Transactions on Geoscience and Remote Sensing, vol. 39, no. 11, pp. 2332-2342, Nov. 2001.

[121] L.F. Famil, E. Pottier, and J.S. Lee, "Unsupervised classification and analysis of natural scenes from polarimetric interferometric SAR data," Proc of IGARSS '01, pp. 27152717, 2001.

[122] J.S. Lee, M.R. Grunes, and E. Pottier, "Quantitative comparison of classification 
capability: fully polarimetric versus dual and single-polarization SAR," IEEE Transactions on Geoscience and Remote Sensing, vol. 39, no. 11, pp. 2343-2351, Nov. 2001.

[123] K.P. Papathanassiou and S.R. Cloude, "Single-baseline polarimetric SAR interferometry," IEEE Transactions on Geoscience and Remote Sensing, vol. 39, no. 11, pp. 2352-2363, Nov. 2001.

[124] D. Kasilingam and J. Shi, "Artificial neural network-based inversion technique for extracting ocean surface wave spectra from SAR images," Proc of IGARSS '97, pp. 1193-1195, 1997.

[125] J.S. Lee, R.W. Jansen, D.L. Schuler, T.L. Ainsworth, G.O. Marmorino, and S.R. Chubb, "Polarimetric analysis and modeling of multifrequency SAR signatures from Gulf Stream fronts," IEEE Journal of Oceanic Engineering, vol. 23, no. 4, pp. 322333, Oct. 1998.

[126] D.L. Schuler, D. Kasilingam, J.S. Lee, R.W. Jansen, and G. De Grandi, "Polarimetric SAR measurements of slope distribution and coherence changes due to internal waves and current fronts," Proc of IGARSS '02, pp. 638-640, 2002.

[127] J.S. Lee, E. Krogager, T.L. Ainsworth, and W.M. Boerner, "Polarimetric analysis of radar signature of a manmade structure," Proc of 2007 Asia-Pacific Microwave Conference, pp. 1-4, 2007.

[128] S.R. Cloude and E. Pottier, "A review of target decomposition theorems in radar polarimetry," IEEE Trans. on Geoscience and Remote Sensing, vol. 34, no. 2, pp. 498518, Mar. 1996.

[129] E. Krogager, "New decomposition of the radar target scattering matrix," Electronics Letters, vol. 26, no. 18, pp. 1525-1527, Aug. 1990.

[130] E. Krogager, "Aspects of Polarimetric Radar Imaging," PhD Dissertation, Technical University of Denmark, 1993.

[131] E. Pottier, "Dr. J. R. Huynen's main contributions in the development of polarimetric radar techniques and how the 'Radar Targets Phenomenological Concept' becomes a theory," Proc. SPIE 1748, Radar Polarimetry, 1993.

[132] W.A. Holm and R.M. Barnes, "On radar polarization mixed target state decomposition techniques," Proc of the 1988 IEEE National Radar Conference, pp. 249-254, 1988. 
[133] S.R. Cloude, "Target decomposition theorems in radar scattering," Electronics Letters, vol. 21, no. 1, pp. 22-24, Jan. 1985.

[134] J.J. van Zyl, "Application of Cloude's target decomposition theorem to polarimetric imaging radar data," Proc. SPIE 1748, Radar Polarimetry, 1993.

[135] A. Freeman and S. Durden, "A three-component scattering model to describe polarimetric SAR data," Proc. SPIE Conf. Radar Polarimetry, 1992.

[136] A. Freeman and S.L. Durden, "A three-component scattering model for polarimetric SAR data," IEEE Transactions on Geoscience and Remote Sensing, vol. 36, no. 3, pp. 963-973, May 1998.

[137] Y. Yamaguchi, T. Moriyama, M. Ishido, and H. Yamada, "Four-component scattering model for polarimetric SAR image decomposition," IEEE Transactions on Geoscience and Remote Sensing, vol. 43, no. 8, pp. 1699-1706, Aug. 2005.

[138] Y. Yamaguchi, Y. Yajima, and H. Yamada, "A four-component decomposition of POLSAR images based on the coherency matrix," IEEE Geoscience and Remote Sensing Letters, vol. 3, no. 3, pp. 292-296, Jul. 2006.

[139] A. Freeman, "Fitting a two-component scattering model to polarimetric SAR data from forests," IEEE Transactions on Geoscience and Remote Sensing, vol. 45, no. 8, pp. 2583-2592, Aug. 2007.

[140] S.R. Cloude and E. Pottier, "An entropy based classification scheme for land applications of polarimetric SAR," IEEE Trans. on Geoscience and Remote Sensing, vol. 35, no. 1, pp. 68-78, Jan. 1997.

[141] A. Simi, G. Manacorda, M. Miniati, S. Bracciali, and A. Buonaccorsi, "Underground asset mapping with dual-frequency dual-polarized GPR massive array," Proc of 13th International Conference on Ground Penetrating Radar, pp. 1-5, 2010.

[142] U. Boniger and J. Tronicke, "Subsurface utility extraction and characterization: combining GPR symmetry and polarization attributes," IEEE Transactions on Geoscience and Remote Sensing, vol. 50, no. 3, pp. 736-746, Mar. 2012.

[143] H. Liu, J. Zhao, and M. Sato, "A hybrid dual-polarization GPR system for detection of linear objects," IEEE Antennas and Wireless Propagation Letters, vol. 14, pp. 317320, Oct. 2015.

[144] Z. Zeng, J. Li, L. Huang, X. Feng, and F. Liu, "Improving target detection accuracy 
based on multipolarization MIMO GPR," IEEE Transactions on Geoscience and Remote Sensing, vol. 53, no. 1, pp. 15-24, Jan. 2015.

[145] T. Moriyama, M. Nakamura, Y. Yamaguchi, H. Yamada, and W.M. Boerner, "Classification of target buried in the underground by radar polarimetry," IEICE Transactions on Communications, vol. E82-B, no. 6, pp. 951-957, Jun. 1999.

[146] T. Moriyama, M. Nakamura, Y. Yamaguchi, H. Yamada, and W.M. Boerner, "Radar polarimetry applied to the classification of target buried in the underground," Proc. SPIE 3120, Wideband Interferometric Sensing and Imaging Polarimetry, 1997.

[147] I.L. Morrow and P. van Genderen, "Effective imaging of buried dielectric objects," IEEE Transactions on Geoscience and Remote Sensing, vol. 40, no. 4, pp. 943-949, Apr. 2002. 